Rowan University

Rowan Digital Works

Theses and Dissertations

$9-4-2019$

\title{
The impact of patient-specific vascular structure on localized cooling in the human heart
}

Nathan Paul Spangenberg

Rowan University

Follow this and additional works at: https://rdw.rowan.edu/etd

Part of the Biomedical Engineering and Bioengineering Commons, and the Mechanical Engineering Commons

\section{Recommended Citation}

Spangenberg, Nathan Paul, "The impact of patient-specific vascular structure on localized cooling in the human heart" (2019). Theses and Dissertations. 2733.

https://rdw.rowan.edu/etd/2733

This Thesis is brought to you for free and open access by Rowan Digital Works. It has been accepted for inclusion in Theses and Dissertations by an authorized administrator of Rowan Digital Works. For more information, please contact graduateresearch@rowan.edu. 


\title{
THE IMPACT OF PATIENT-SPECIFIC VASCULAR STRUCTURE ON LOCALIZED COOLING IN THE HUMAN HEART
}

\author{
By \\ Nathan Paul Spangenberg
}

\author{
A Thesis \\ Submitted to the Department of Mechanical Engineering \\ College of Engineering \\ In partial fulfillment of the requirement \\ For the degree of \\ Master of Science in Mechanical Engineering \\ at \\ Rowan University \\ December 14, 2018
}

Thesis Advisor: Thomas Lad Merrill, Ph.D. 
(C) 2018 Nathan P. Spangenberg 


\section{Dedications}

To Jesus Christ, my Lord and Savior. For graciously giving me the opportunity, resilience, and strength to pursue this research effort in hopes of helping to improve therapies used to treat patients with AMI. Your unconditional love and guidance in my life continues to amaze me each and every day.

To my wife, Sarah Elizabeth. For your unconditional love and support throughout all my academic endeavors. I am overwhelmed by your love and encouraging spirit, and it means the world to me having you by my side. I love you. 


\section{Acknowledgments}

My deepest appreciation is to my committee advisor, Dr. Thomas Merrill, for his inspiration, encouragement, and enthusiasm he has so graciously dedicated to my work. His instruction, leadership, and 4:00am emails have made me most thankful to have been given the opportunity to be a part of his research team. I also wish to acknowledge the effort of the research team at FocalCool, LLC, specifically my committee member Jennifer E. Mitchell, and colleagues, Joseph R. Licwinco, Denise Merrill, and Nesrine Bouhira. My work has richly benefited from your help and support, and for that I extend my deepest appreciation. I also wish to thank the financial support from the National Institutes of Health (NIH) by providing the grants necessary to fund this work.

The support, encouragement, and conversations I've had from my other committee members, Dr. Francis (Mac) Haas (Department of Mechanical Engineering, Rowan University, Glassboro, NJ), and Dr. Anilchandra Attaluri (Department of Mechanical Engineering, Penn State University, Harrisburg, PA) has been invaluable to me. Thank you for the time you so willingly invested in this work.

This work would not have been possible without the DICOM data sets of patientspecific cardiovascular models provided from Dr. Michael Bateman and Dr. Tinen Iles (Visible Heart® Laboratory, University of Minnesota, Minneapolis, MN). Their collaboration efforts and enthusiasm for this research has richly benefitted the progress of this work. Thank you to you both for your invested time and valuable partnership.

My sincere appreciation is to my parents, Stephen and Maribeth Spangenberg, who continually provided their love, financial, and spiritual support. And to my family, for their encouragement and moral support. You have all been an inspiration by always motivating me to persevere in all things. A simple thank you just isn't enough. 


\begin{abstract}
Nathan Paul Spangenberg

THE IMPACT OF PATIENT-SPECIFIC VASCULAR STRUCTURE ON LOCALIZED COOLING IN THE HUMAN HEART

2017-2018

Thomas L. Merrill, Ph.D.

Master of Science in Mechanical Engineering
\end{abstract}

Acute Myocardial Infarction (AMI) is the leading cause of worldwide death and disability, and approximately 720,000 Americans will experience an AMI in 2018. Studies have shown that rapid hypothermia therapy $\left(<35^{\circ} \mathrm{C}\right)$ before reperfusion in patients with AMI can reduce infarct size by $37 \%$. Localized therapeutic hypothermia has proven the potential to cool heart tissue rapidly following $\mathrm{AMI}, 3^{\circ} \mathrm{C}$ in 5 minutes. Using Materialise Mimics digital imaging software and the finite volume method we analyzed temperature distributions in six patient-specific left main coronary artery (LMCA) models. A mock circulatory loop was used to determine the exiting temperatures of a standard 7 Fr catheter to feed into our model with flow rates ranging from $29.2 \mathrm{ml} / \mathrm{min}$ to $68.85 \mathrm{ml} / \mathrm{min}$. Our work showed that therapeutic hypothermia $(\mathrm{TH})$ temperatures were evident at the outlets of three out of all six heart models, which varied in each left anterior descending (LAD) and left circumflex (LCX) artery depending on flowrate. Results of this study indicate that biovariability in patient-specific vascular structures significantly impacts therapeutic hypothermia (TH) treatment methods. These results indicate that further research is needed to examine more accurate physiological effects, such as pulsatile flow and vessel wall thickness. Future models will be used to provide insight to guide more efficient TH device designs and operation parameters to optimize patient outcomes following AMI. 


\section{Table of Contents}

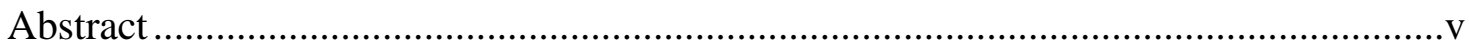

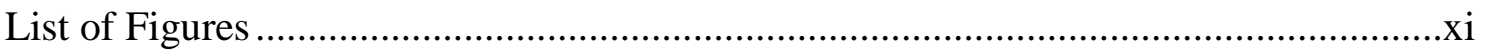

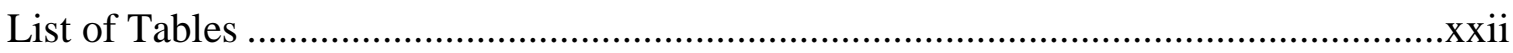

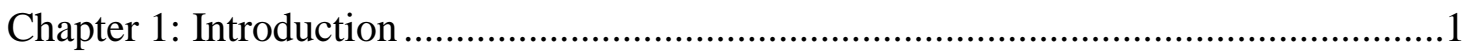

Basic Physiology of the Human Heart ....................................................................

Vascular Networks ..........................................................................

Coronary Flow Rates ......................................................................

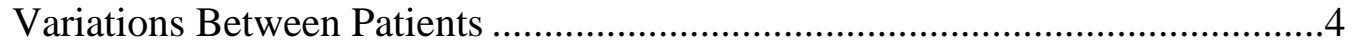

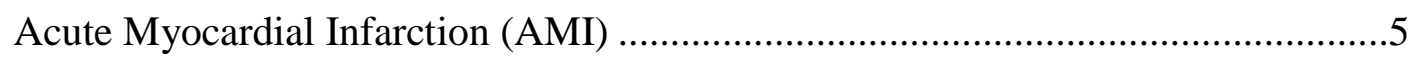

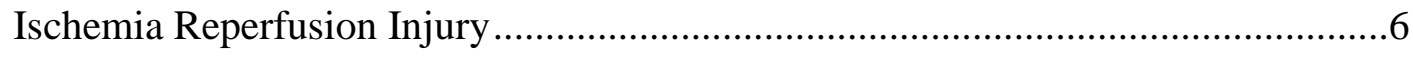

Existing Treatments ........................................................................

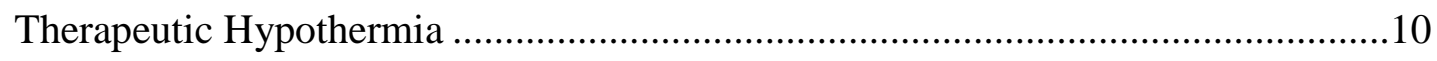

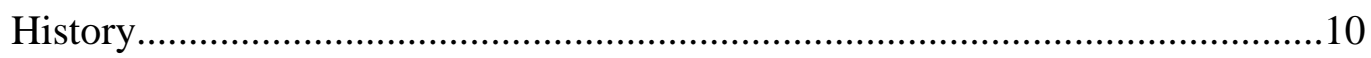

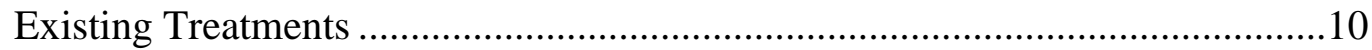

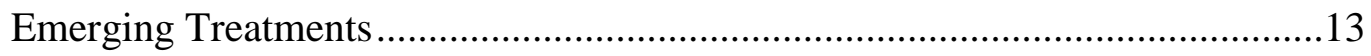

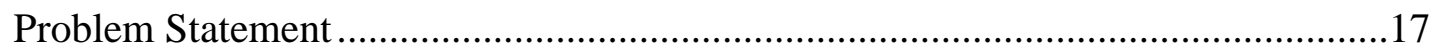

Significance of Work ............................................................................... 17

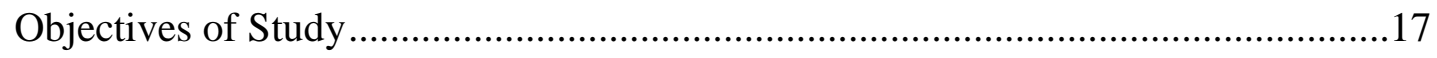

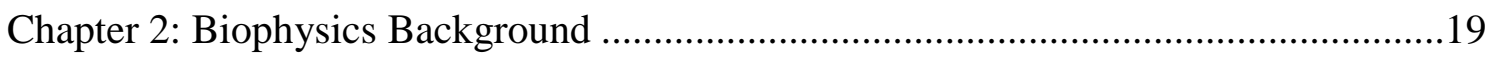

Laminar Flow: Conservation of Linear Momentum ............................................19

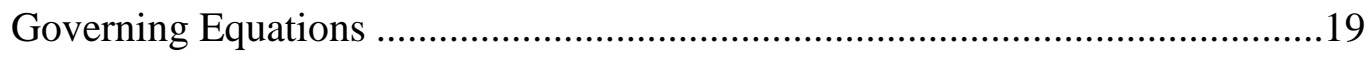

Laminar Flow: Conservation of Thermal Energy with Constant Wall Temperatures

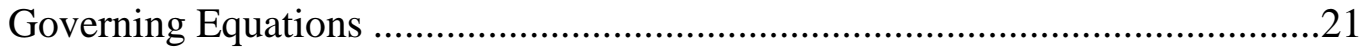




\section{Table of Contents (Continued)}

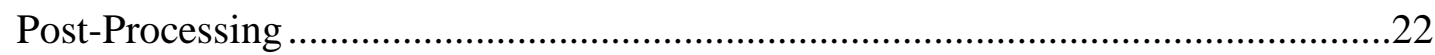

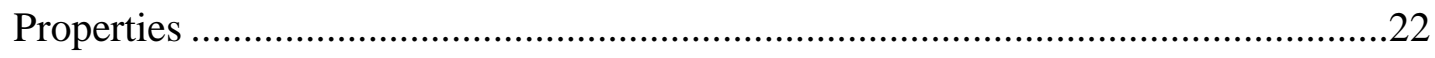

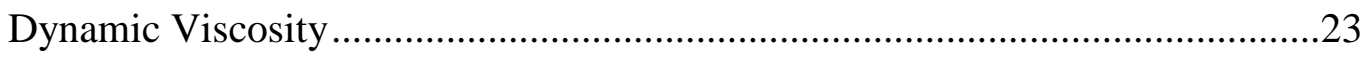

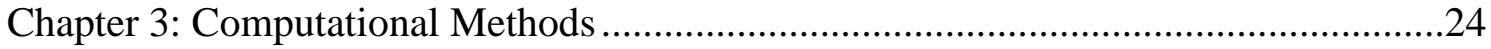

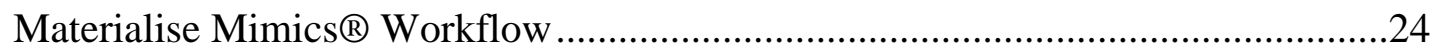

DICOM Image Sets to 3D Vasculature Creation................................................24

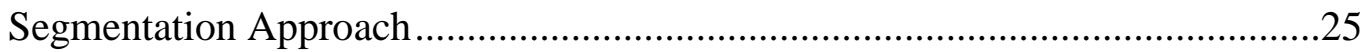

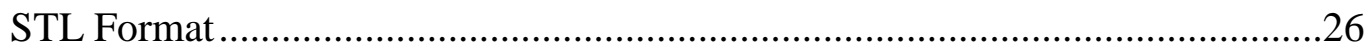

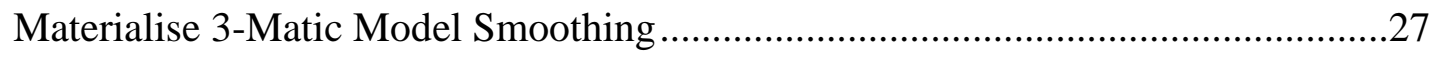

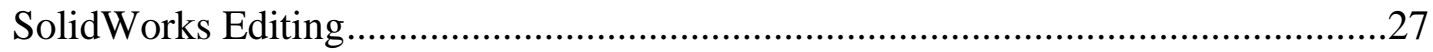

Brief Description of Siemens STAR-CCM+ Workflow.......................................29

Finite Volume Method ...........................................................................29

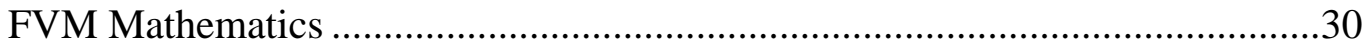

Boundary Conditions Applied to FVM ........................................................30

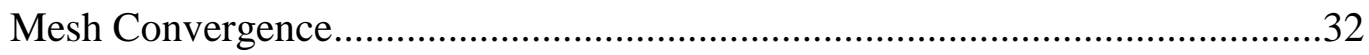

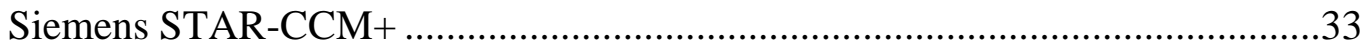

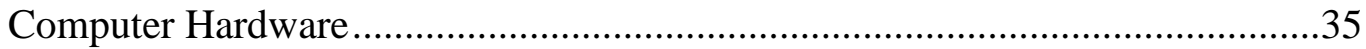

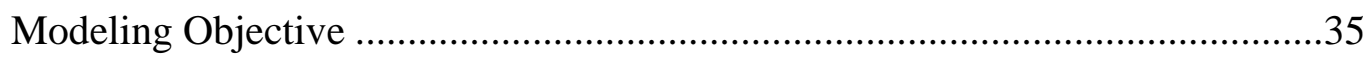

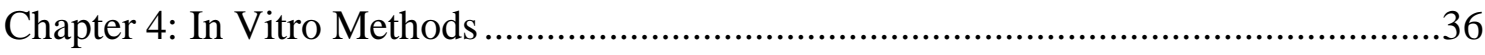

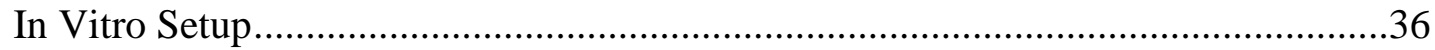

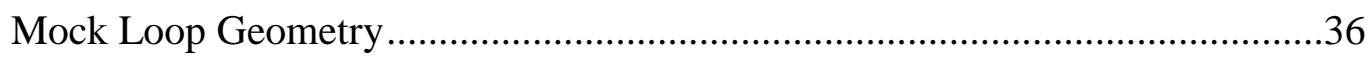

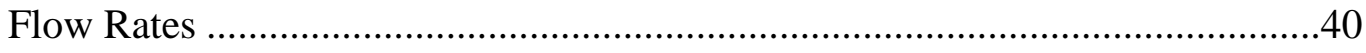

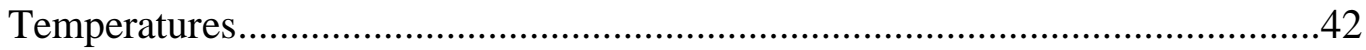




\section{Table of Contents (Continued)}

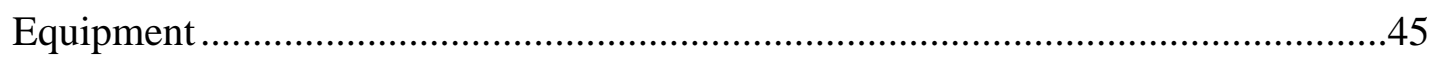

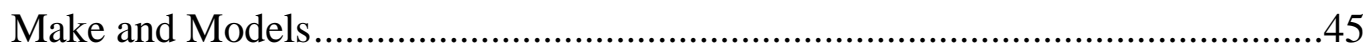

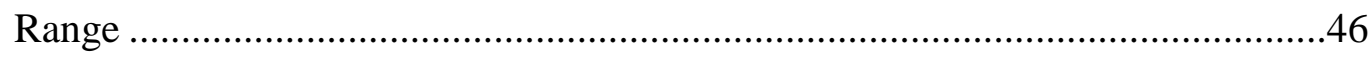

Calibration and Precision ........................................................................4

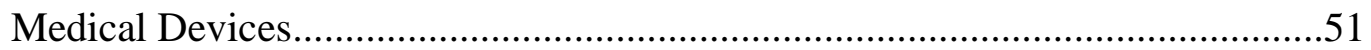

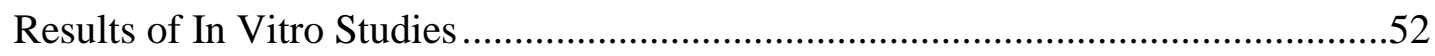

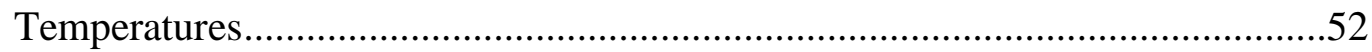

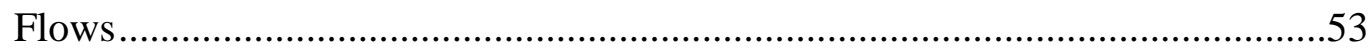

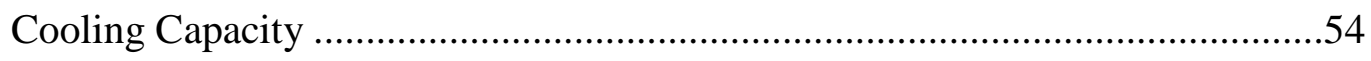

Summary and Connection to Computational Work ............................................56

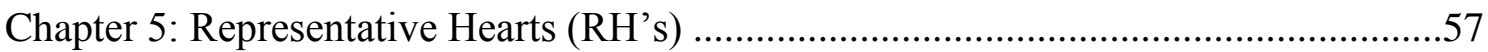

Source of Human Heart 3D Models.....................................................................57

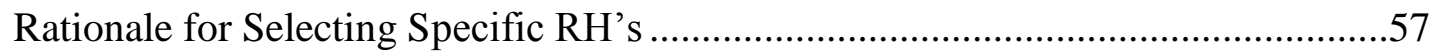

Heart Model Structural Comparison ...............................................................60

Quantify Differences Between Models.........................................................60

Chapter 6: Thermal Fluid Model Setup for RHs ...................................................68

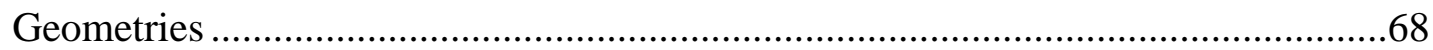

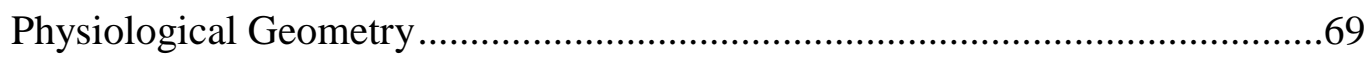

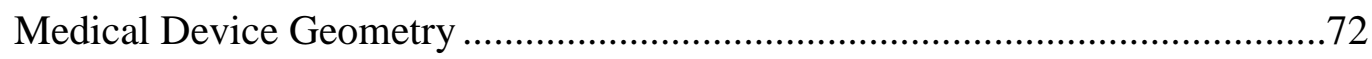

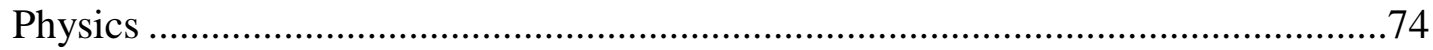

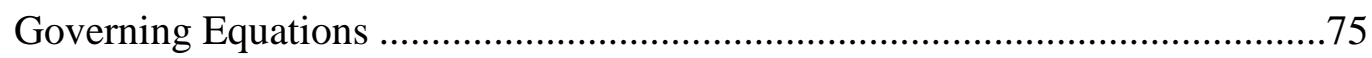

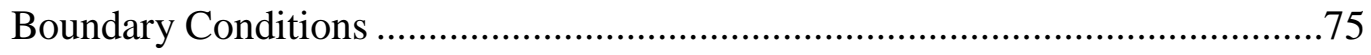

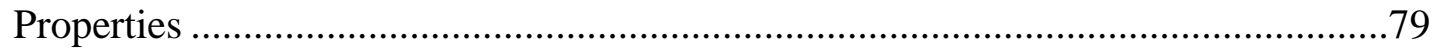




\section{Table of Contents (Continued)}

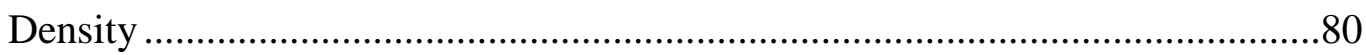

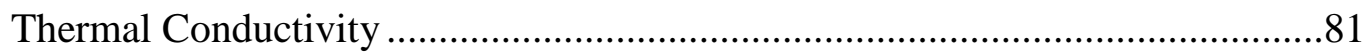

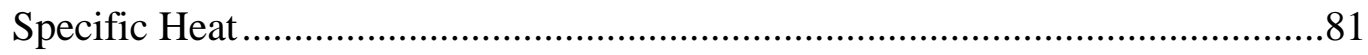

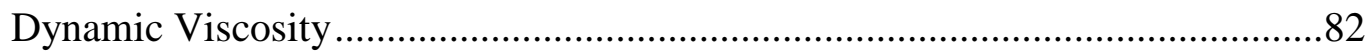

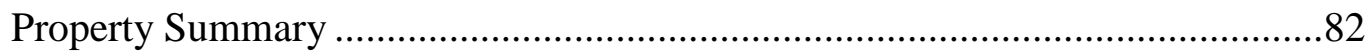

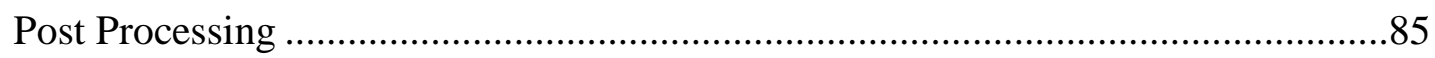

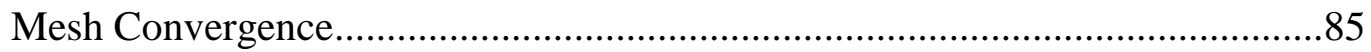

Thermal Fluid Model Testing Matrix ................................................................ 90

Chapter 7: Thermal Fluid Model Predictions .......................................................... 93

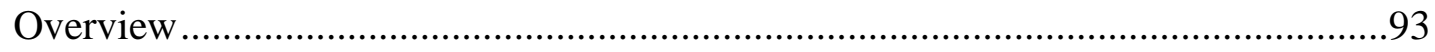

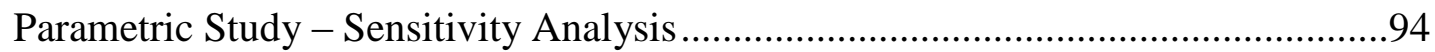

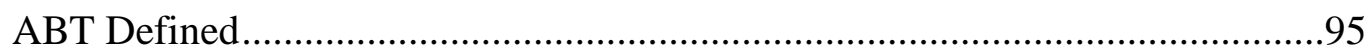

ABT in Patient-Specific Vascular Structures ................................................96

ABT in Single Heart with Single Flow Rate ................................................99

ABT in All Hearts with Single Flow Rate .....................................................101

ABT in Single Heart with Multiple Flow Rates ..............................................103

ABT in All Hearts with Multiple Flow Rates .................................................107

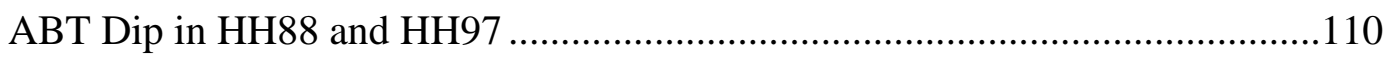

Impact of TH Device Location Placement on Cooling Effectiveness .................118

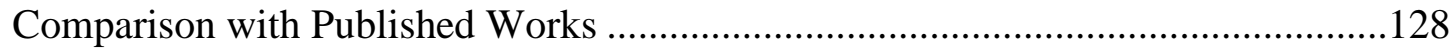

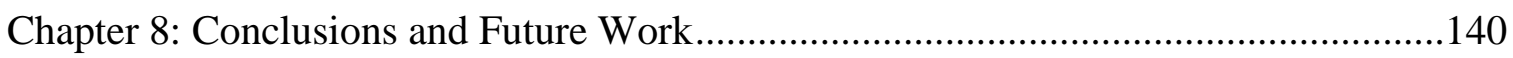

Approaches and Recommendations for Cooling Hearts ......................................140

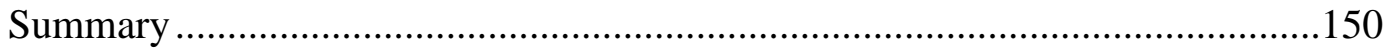




\section{Table of Contents (Continued)}

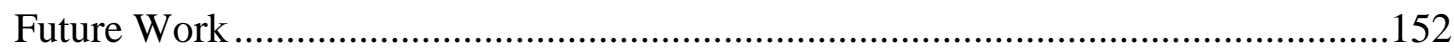

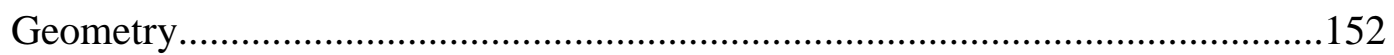

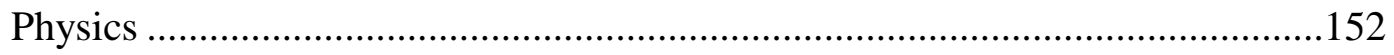

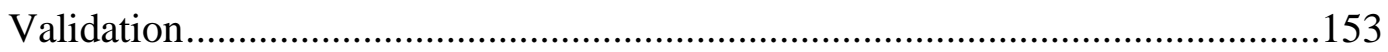

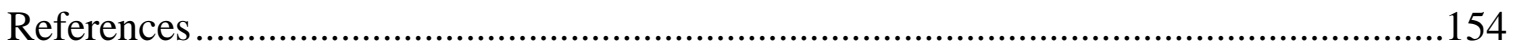

Appendix A: Simplified Explanation of Fully Developed Velocity Profile, Energy

Balance, and the Temperature Dependent Heat Source Equation ..................................166

Appendix B: Description of Viscosity Entered as Function of Temperature in STAR-

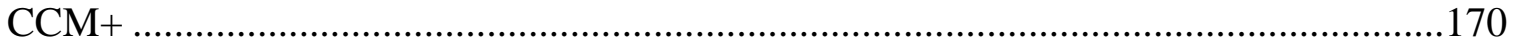

Appendix C: Extension of the Thermal Fluid Modeling Matrix Specifications..............173

Appendix D: Qualitative Analysis of How ABT Changes Along the Fluid Flow Path

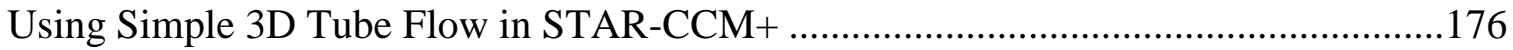


Figure

\section{List of Figures}

Figure 1.1. Basic cardiac circulation of the human heart. The right side of the heart
supplies blood to pulmonary circulation, while the left side of the heart

Figure 1.1. Basic cardiac circulation of the human heart. The right side of the heart
supplies blood to pulmonary circulation, while the left side of the heart supplies blood to systemic circulation [3]. Image of the LMCA bifurcating into the LAD and LCX arteries that each branch into daughter vessels producing patient-specific artery networks. Each person has a unique coronary vascular network with arteries varying in length, diameter, and location of bifurcated branches [5]. This work will explore organ cooling along these vessels

Figure 1.3. The cardiac cycle for blood flow entering the left coronary artery [17]. The typical cardiac cycle is pulsatile in nature and consists of the systole ejection phase (SEP) and the diastole phase (DP), which represents the time between $0.3 \mathrm{~s}$ to $0.5 \mathrm{~s}$ and $0.5 \mathrm{~s}$ to the beginning of the SEP cycle, respectively ..........4

Figure 1.4. Schematic of left anterior descending artery occluded with plaque build-up. Purple area distal to occluded artery represents myocardial area at risk of infarction [27]. Therapeutic hypothermia is a promising treatment option that may reduce tissue damage and improve patient outcomes

Figure 1.5. Primary percutaneous coronary intervention (P-PCI) performed using balloon angioplasty with stenting [51]. The occlusion is located in the artery network (a) and a guide is advanced through the femoral artery into the occluded vessel with the balloon and stent (b). The balloon is inflated (c) to expand the stent (d) and open the occluded vessel [25]

Figure 1.6. Endovascular cooling device inserted in the femoral vein and positioned in the Inferior Vena Cava (IVC). Patients undergoing endovascular cooling interventions experience whole-body hypothermia at $33^{\circ} \mathrm{C}$ [13]. Endovascular cooling methods maintain target $\mathrm{TH}$ temperatures more efficiently than conventional external cooling but are unable to reach $\mathrm{TH}$ temperatures quicker [54]

Figure 1.7. (a) 3D representation of the left anterior descending artery modeled in Mimics from DICOM HH63 (HH = Human Heart) from the Visible Heart Lab, University of Minnesota. (b) Schematic illustration of a standard guide catheter delivering cooled saline into left coronary artery via femoral artery access. 
Figure 3.1. 3D volume rendering window in Mimics ${ }^{\circledR}$ of the LMCA segmented from 2D CT angiography image scans of HH55. The views in the window pane consists of the coronal (a), axial (b), sagittal (c), and the 3D rendering window (d). Green highlighted pixels (c) represent the segmented blood flow path of the artery network. The fully segmented 3D model (d) represents a patient-specific structure that can be exported in STL format and used in CFD simulations for thermal-fluid flow analysis

Figure 3.2. DICOM to SolidWorks editing workflow. DICOM images were attained from the Visible Heart Lab, University of Minnesota (Step 1) and imported into Mimics for 3D model segmentation and 3-matic smoothing (Step 2). The Mimics/3-matic models were exported in binary STL format (Step 3) and imported into SolidWorks for inlet/outlet trimming and creation of the proximal GuideLiner® insertion (Step 4)

Figure 3.3. With an STL file formed by Mimics and the original DICOM data set, a 3D modeling and editing tool, SolidWorks ${ }^{\circledR}$ was used to orthogonally trim the inlet and outlets of each LAD and LCX artery model. The following HH55 LCX model contains a single inlet and 33 outlets, however, the number of outlets varied with each LAD and LCX model employed in this work ........28

Figure 3.4. The physics space (1) in a simple bent tube is comprised of a grid that encompasses the computational domain. At each node (2), the governing equations in algebraic form are created. If we combine 1 and 2, a system of equations can be used in STAR-CCM+ to solve the flow field [129]

Figure 3.5. Mesh convergence analysis comparing the number of cells in a mesh and a corresponding $\mathrm{ABT}$ on a cross sectional plane.....

Figure 3.6. The general CFD modeling approach that describes the necessary steps for preparing and appropriately executing a CFD simulation [114]

Figure 4.1. Schematic of modified coronary mock loop showing catheter path and mock vessels [93]. The guide (represented by solid blue line) is inserted into the femoral insertion sheath and advanced through the glass aorta into the LMCA ostium. The table at the bottom of the schematic describes the sizes and proper flow rates for vessels of interest 
Figure 4.2. Image of coronary mock loop showing catheter path and mock vessels. Note: Thermocouple (TC), common carotid artery (CCA), and left main coronary artery (LMCA). Flow from the large reservoir is pumped through a flow meter before entering the Tygon tubing as proximal Aorta flow. Flow enters the Aortic arch and disperses into the coronary artery ostia where it continues to the left main coronary artery (LMCA) and the guide extension outlet. Aortic flow exits through the common carotid artery (CCA) and femoral arteries downstream

Figure 4.3. Continuation of Fig. 4.2 showing catheter path and mock vessels. Distilled water is heated in the reservoir and pumped into circulation via the peristaltic pump. Flow to the common carotid artery (CCA) and left main coronary artery (LMCA) can be adjusted using flow control valves downstream.......40

Figure 4.4. The Cordis ${ }^{\circledR}$ catheter enters the ostium of the LMCA and the guide extension is advanced $8.5 \mathrm{~cm}$ through the Tuohy borst adapter (TB Adapter), out of the catheter, and positioned in tight fit tubing. The fittings and catheter are insulated (not shown) after assembly. The "To Reservoir" label represents flow advancing towards the LMCA flow meter and into the mock loop reservoir. The "To Graduated Cylinder" label refers to infusate flowing towards the reservoir (or graduated cylinder), which is used to measure guide extension flowrate

Figure 4.5. Internal view of custom built portable cooling console. The peristaltic pump (a) circulates flow from a small reservoir to the heat exchanger (b). Distilled water is chilled through the heat exchanger and advanced into the mock circulatory loop (4) by way of the Guide Catheter

Figure 4.6. Snapshot of the calibration design of experiments for thermocouple, flow, and pressure calibration with each corresponding input, output, parameter, and variable

Figure 4.7. Temperatures entering the Cordis ${ }^{\circledR}$ guide and exiting the guide extension vs infusate flow rates from mock loop cooling console. Increased infusate flowrates resulted in lower fluid temperatures both entering and exiting the Cordis ${ }^{\circledR}$ guide and guide extension, respectively. Infusate flowrates leaving the heat exchanger varied between $0.023^{\circ} \mathrm{C}$ and $0.112^{\circ} \mathrm{C}$. Standard. Note: standard deviation error bars are capped

Figure 4.8. Cordis ${ }^{\circledR} 7 \mathrm{~F}$ guide cooling capacity for flowrate vs outlet temperature. Blue circles represent the power (W) required to produce guide extension outlet temperatures at corresponding flowrates. 
Figure 5.1. Proximal LAD and LCX GuideLiner® insertion method. Large proximal artery diameters are critical for accommodating and simulating GuideLiner® insertion.

Figure 5.2. Representative hearts $(\mathrm{RH})$ that were chosen for this work. These RH's were created in Mimics ${ }^{\circledR}$ and were exported into STAR-CCM+ for CFD modeling. All RH models contain a LMCA stem that bifurcates into an LAD and LCX artery ..... .59

Figure 5.3. The proximal segments of the LAD and LCX arteries highlighted in yellow.

Note: proximal $\mathrm{LAD}=\mathrm{Pl}$ and proximal $\mathrm{LCX}=\mathrm{PX}$ .61

Figure 5.4. Variations in proximal LCX artery lengths. The proximal LCX extends from the left main bifurcation to the first obtuse marginal branch (FOM). HH88 (a) contains the shortest proximal segment length, while HH259 (b) and HH178 (c) contain the average and longest proximal segment lengths of all heart models chosen for this work, respectively

Figure 5.5. Quantitative plot showing the comparison between surface area and all LAD and LCX representative heart $(\mathrm{RH})$ models .64

Figure 5.6. Quantitative plot showing the comparison between volume and all LAD and LCX representative heart $(\mathrm{RH})$ models.... .64

Figure 5.7. Surface area to volume ratios between all LAD and LCX representative heart (RH) models

Figure 6.1. Ideal LAD (a) and LCX (b) networks [143]. Note for the LAD (a): first diagonal branch (1DB), second diagonal branch (2DB), septal branches (SB), and anterior descending (AD). Note for the LCX: first obtuse marginal branch (1om), second obtuse marginal branch (2om), and posterolateral branch (Plb)

Figure 6.2. Isometric view of a general representation of the OD and ID of the GuideLiner® in a straight tube describing the GuideLiner® boundaries for each proximal artery. The depth of the insertion varied between $3-5 \mathrm{~mm}$ and the OD and ID were fixed at $1.9 \mathrm{~mm}$ and $1.57 \mathrm{~mm}$, respectively....

Figure 6.3. Patient-specific LAD networks for HH55, HH88, HH97, HH102, HH178, and $\mathrm{HH} 259$, for hearts a. through f., respectively. Note: first diagonal branch $=$ $1 \mathrm{DB}$, second diagonal branch $=2 \mathrm{DB}$, septal branches $=\mathrm{SB}$, and the anterior descending branch $=$ AD. HH88 had no identifiable septal perforator branch (b), which could have been attributed to poor image quality in the DICOM file format . .70 
Figure

\section{List of Figures (Continued)}

Figure 6.4. Patient-specific LCX networks for HH55 (a), HH88 (b), HH97 (c), HH102 (d), HH178 (e), and HH259 (f). Note: the first obtuse marginal branch = 1om, second obtuse marginal $=2 \mathrm{om}$, and the posterolateral branch $=$ Plb. HH97 appears to have a third obtuse marginal branch (c) that extends between the second obtuse marginal and the posterolateral branches [144]....

Figure 6.5. Sagittal view of a general representation of the inlet geometry and boundaries describing the GuideLiner ${ }^{\circledR}$ placed in each proximal LMCA. The depth of the insertion varied between $3-5 \mathrm{~mm}$ and the OD and ID were fixed at $1.9 \mathrm{~mm}$ and $1.57 \mathrm{~mm}$, respectively

Figure 6.6. HH55 LAD Navier Stokes and thermal energy boundary conditions applied to all patient-specific RH models. Identical boundary conditions were applied to each of the remaining LAD and LCX artery models. Each heart model exhibits a single blood flow inlet and GuideLiner ${ }^{\circledR}$ flow inlet. The number of outlets for each heart model varied and ranged from 7 outlets for HH88 LAD to 33 outlets for HH55 LCX. Navier-Stokes description is designated to left of comment (slash), while thermal energy is represented to the right ..........76

Figure 6.7. Eccentric GuideLiner ${ }^{\circledR}$ catheter in the proximal HH55 LAD representing two distinct inlets for the GuideLiner® and blood flow. Inlet flow conditions for the blood and GuideLiner® lead to assumed complete mixing solution of blood and distilled water....

Figure 6.8. Average left coronary artery blood flow as determined in literature using various methods involving lumped parameter modeling, 3D epicardium simulations, ultrasound, and phase contrast MRI. Based on these published findings, a total inlet flow rate of $75 \mathrm{~mL} / \mathrm{min}$ was chosen for all of our CFD simulations

Figure 6.9. Total inlet flow rate representing GuideLiner ${ }^{\circledR}$ and blood flow entering each proximal LAD and LCX artery network .78

Figure 6.10. Theoretical description of how mixture properties for blood and distilled water were determined for the CFD inlet boundary conditions. $\mathrm{T}=\mathrm{a}, \mathrm{b}, \mathrm{c}$ represents the guide extension inlet temperatures $29.4,23.16$, and $19.2^{\circ} \mathrm{C}$ with corresponding flow rates $29.2,49.03,68.85 \mathrm{ml} / \mathrm{min}$, respectively. Blood and distilled water was mixed at $\mathrm{T}=\mathrm{a}, \mathrm{b}, \mathrm{c}$ guide extension infusate temperatures, and new mixed blood and infusate properties were used as inlet conditions for the CFD models.

Figure 6.11. A close-up of the cells that encompass the polyhedral mesh (a) and a closeup of the 3 prism layers formed at each wall boundary (b). The prism layers are fixed at the fluid-artery wall interface so that no-slip wall boundary conditions could be accurately solved. 
Figure 6.12. A total of six base sizes were chosen for each heart model and the resultant number of cells were plotted against the ABT (a) at a cross sectional plane (CSP) (c) in the artery network. A proximal mesh view (b) shows the cells lining the surface of the HH178 LAD model. A base size of $0.00015 \mathrm{~m}$ resulted in a reasonable number of cells $(\sim 8 \mathrm{e} 5)$ that led to invariant average bulk temperature at the CSP

Figure 6.13. Six base sizes were chosen for heart HH88 LCX and the resultant number of cells were plotted against the ABT (a) at a cross sectional plane (CSP) (b). A proximal mesh view (b) shows the cells lining the surface of the HH88 LCX model. A base size of $0.00015 \mathrm{~m}$ also produced a mesh with ( 6e5) cells that yielded sufficient ABT results.

Figure 7.1. The blood temperature as it navigates through blood vessels of varying diameters, as proposed by Chen and Homes [149]. The dashed line indicates the range of the tissue temperature surrounding the vessels. Most temperature changes occur in smaller vessels, whereas large vessels retain blood flow path temperatures for longer distances.

Figure 7.2. Cross-sectional planes (C-SP's) spaced 5mm apart extending from HH259 LAD inlet to the outlet. The mother vessel for each LAD human heart $(\mathrm{HH})$ model originated at the proximal inlet and extended to each distal LAD outlet

Figure 7.3. Cross-sectional planes (C-SP's) spaced 5mm apart extending from HH259 LCX inlet to the outlet. The mother vessel for each LCX human heart (HH) model originated at the proximal inlet and extended to each distal posterolateral branch outlet.

Figure 7.4. Cooling effectiveness (CE) represents the ratio of the distance from the proximal inlet where TH-ABTs are evident in the mother vessel over the entire length of the mother vessel. If TH-ABTs are evident up to and including half the length of the vessel, then this vessel would have $50 \% \mathrm{CE}$. Furthermore, if TH-ABTs were measured at the outlet, then the vessel would have $100 \% \mathrm{CE}$

Figure 7.5. ABT along the LAD mother vessel flow path with single GuideLiner ${ }^{\circledR}$ flow rate at $29.2 \mathrm{~mL} / \mathrm{min}$ for $\mathrm{HH} 178$.

Figure 7.6. ABT along the LCX mother vessel flow path with single GuideLiner@ flow rate at $29.2 \mathrm{~mL} / \mathrm{min}$ for $\mathrm{HH} 178$.

Figure 7.7. All LAD representative heart models using a $29.4^{\circ} \mathrm{C}$ infusate fluid mixture at a flowrate of $29.2 \mathrm{ml} / \mathrm{min}$. Temperature drop in HH88 and HH97, at locations of about $30-40 \mathrm{~mm}$, are discussed in detail in the next section. 
Figure 7.8. All LCX representative heart models using a $29.4^{\circ} \mathrm{C}$ infusate fluid mixture at a flowrate of $29.2 \mathrm{ml} / \mathrm{min}$

Figure 7.9. Results of ABT at each cross-sectional plane location for HH259 LAD using flow rates obtained from in vitro mock loop study; $29.2 \mathrm{ml} / \mathrm{min}, 49.03$ $\mathrm{ml} / \mathrm{min}$, and $68.85 \mathrm{ml} / \mathrm{min}$. The rapid spike in ABT at the $40 \mathrm{~mm}$ plane is the result of a bifurcated septal perforator branch that deviated from the mother vessel.

Figure 7.10. Results of ABT vs cross-sectional plane location for HH259 LCX using flow rates obtained from in vitro mock loop study; $29.2 \mathrm{ml} / \mathrm{min}, 49.03 \mathrm{ml} / \mathrm{min}$, and $68.85 \mathrm{ml} / \mathrm{min}$. The rapid spike in ABT at the $70 \mathrm{~mm}$ plane resulted in a bifurcation from the second obtuse marginal branch that deviated from the mother vessel.....

Figure 7.11. Cross-sectional area of the $135 \mathrm{~mm}$ cross-sectional plane from HH259 LAD. Note: Cross-sectional area: $1.935 \mathrm{e}-2 \mathrm{~mm}^{2}$. Average bulk temperature (ABT): $36.55^{\circ} \mathrm{C}$ at $68.85 \mathrm{ml} / \mathrm{min}$ infusate flow..... 106

Figure 7.12. Cross-sectional area of the $135 \mathrm{~mm}$ cross-sectional plane from HH259 LCX. Note: Cross-sectional area: $1.968 \mathrm{e}-2 \mathrm{~mm}^{2}$. Average bulk temperature (ABT): $36.12^{\circ} \mathrm{C}$ at $68.85 \mathrm{ml} / \mathrm{min}$ infusate flow.....

Figure 7.13. The impact of all six $\mathrm{HH}$ models on $\mathrm{CE}$ with their corresponding GuideLiner® flow rates in each LAD mother vessel

Figure 7.14. The impact of all six $\mathrm{HH}$ models on $\mathrm{CE}$ with their corresponding GuideLiner ${ }^{\circledR}$ flow rates in each LCX mother vessel

Figure 7.15. ABT distribution along mother vessel of patient HH88 LAD with artifact issue occurring between planes $30 \mathrm{~mm}$ and $35 \mathrm{~mm}$, at $29.2 \mathrm{ml} / \mathrm{min}$. The ABT steadily increases until the thermal fluid flow path exits the LAD outlet ..111

Figure 7.16. ABT distribution along flow path of patient HH97 LAD with numerous artifact issues originating at the $20 \mathrm{~mm}$ plane. GuideLiner ${ }^{\circledR}$ infusate flow rate: $29.2 \mathrm{ml} / \mathrm{min}$

Figure 7.17. Continuation of planes in $5 \mathrm{~mm}$ increments along each bifurcated branch that had deviated from the mother vessel in HH88 LAD

Figure 7.18. HH88 LAD bifurcations of $35 \mathrm{~mm}$ branches A, B, and C (a). The majority of chilled infusate flowed into branch $A$ and only a minute amount passed into branches B and C. The cross-sectional $35 \mathrm{~mm}$ ABT planes (b) are summed together in STAR-CCM+ using a form of Eq. 7.1 to produce combined ABT values 
Figure 7.19. HH88 LAD with $29.2 \mathrm{ml} / \mathrm{min}$ proximal infusate flow comparing the ABT planes for branches $\mathrm{A}, \mathrm{B}$, and $\mathrm{C}$

Figure 7.20. GuideLiner® flow rate $(29.2 \mathrm{~mL} / \mathrm{min})$ in HH88 LAD with no impact of flow stratification using ABT summation from branches A, B, and C. The ABT's for each corresponding distance were summed together to determine a final $\mathrm{ABT}$ at each distance extending down the artery network

Figure 7.21. Continuation of planes in $5 \mathrm{~mm}$ increments along each bifurcated branch that had deviated from the mother vessel in HH97 LAD.

Figure 7.22. HH97 ABT's comparing the vessel pathway of interest vs the combined ABT for all branches between the GuideLiner® inlet and the $35 \mathrm{~mm}$ plane.

Figure 7.23. Temperature distributions executed in STAR-CCM+ for all patient-specific LAD models using a GuideLiner® flow rate of $29.2 \mathrm{ml} / \mathrm{min}$ at $29.4^{\circ} \mathrm{C}$ in all models

Figure 7.24. Temperature distributions executed in STAR-CCM+ for all patient-specific LCX heart models using a GuideLiner ${ }^{\circledR}$ flow rate of $29.2 \mathrm{ml} / \mathrm{min}$ at $29.4^{\circ} \mathrm{C}$ in all models.

Figure 7.25. Impact of proximal GuideLiner ${ }^{\circledR}$ placement in the LAD and LCX for HH55 using $29.2 \mathrm{ml} / \mathrm{min}$ and $68.85 \mathrm{ml} / \mathrm{min}$ flowrates. ABT's below black-dotted line represent effective therapeutic hypothermia cooling $\left(<35^{\circ} \mathrm{C}\right)$

Figure 7.26. Impact of proximal GuideLiner ${ }^{\circledR}$ placement in the LAD and LCX for HH178 using $29.2 \mathrm{ml} / \mathrm{min}$ and $68.85 \mathrm{ml} / \mathrm{min}$ flowrates. ABT's below blackdotted line represent effective therapeutic hypothermia cooling $\left(<35^{\circ} \mathrm{C}\right) \ldots 119$

Figure 7.27. Impact of surface area to volume $(\mathrm{SA}: \mathrm{V})$ ratios on cooling effectiveness in the LAD pathway of interest for each representative heart $(\mathrm{RH})$ model. Cooling effectiveness is the ratio of the length of TH-ABT in the mother vessel over the entire length of the mother vessel. Therapeutic hypothermia average bulk temperature (TH-ABT) represents the distance from the inlet up to and including the plane listed where TH temperature conditions exist $\left(<35^{\circ} \mathrm{C}\right)$. In general, smaller $\mathrm{SA}: \mathrm{V}$ ratios lead to deeper cooling, as would be expected. Note: SA:V ratio values are listed from left to right in ascending order. 
Figure 7.28. Impact of surface area to volume (SA:V) ratios on cooling effectiveness in the LCX pathway of interest for each representative heart $(\mathrm{RH})$ model. Cooling effectiveness is the ratio of the length of TH-ABT in the mother vessel over the entire length of the mother vessel. Therapeutic hypothermia average bulk temperature (TH-ABT) represents the distance from the inlet up to and including the plane listed where TH temperature conditions exist $\left(<35^{\circ} \mathrm{C}\right)$.

Figure 7.29. Sensitivity of proximal length to mother vessel length for each HH LAD model. Proximal length revealed significant sensitivity to cooling effectiveness in HH88 and HH102, as the two longest proximal length revealed the greatest cooling effectiveness. However, no significant sensitivity was determined between the remaining $\mathrm{HH}$ models

Figure 7.30. Sensitivity of proximal length to mother vessel length for each HH LCX model. Proximal length revealed no significant sensitivity to cooling effectiveness since the longest (HH178) and shortest (HH55) proximal artery lengths played very little impact on cooling effectiveness, as compared to HH97 and HH102

Figure 7.31. Characterization of vascular networks for HH88, HH97, and HH259 LCX represented using $68.85 \mathrm{~mL} / \mathrm{min}$ GuideLiner® flow rates. First obtuse marginal (1om), second obtuse marginal (2ob), and posterolateral (Plb) branches were characterized using Eq. 7.1. Total cooling $\eta_{\mathrm{TC}}$ in HH97 resulted in $97.63 \% \mathrm{CE}$ in the artery network, while $\mathrm{HH} 88$ and $\mathrm{HH} 259$ resulted in $85.26 \%$ and $76.02 \%$ total $\mathrm{CE}$ in each vascular network, respectively

Figure 7.32. Analyses of total network cooling effectiveness $\left(\eta_{T C}\right)$ for each HH model compared to SA:V • total network length. Triangle markers represent mother vessel cooling effectiveness $(L e / L)$ for each $\mathrm{HH}$ model at $68.85 \mathrm{~mL} / \mathrm{min}$ GuideLiner ${ }^{\circledR}$ flow rate, while circles represent SA:V • total network length for each $\mathrm{HH}$ model. A significant reduction in total network cooling effectiveness is evident when SA:V • total network length ratios exceed 700. Note: red dotted line represents 700 cut-off value between good and bad heart cooling candidates.

Figure 7.33. Structural-thermal relationship in HH88 LAD for $49.03 \mathrm{ml} / \mathrm{min}$ guide extension flow rate. Note proximal guide extension inlet temperature is $23.16^{\circ} \mathrm{C}$, and $\mathrm{LAD}$ outlet temperature is $34.13^{\circ} \mathrm{C}$.

Figure 7.34. Structural-thermal relationship in HH88 LAD for $68.85 \mathrm{ml} / \mathrm{min}$ guide extension flow rate. Note: proximal guide extension inlet temperature is $19.2^{\circ} \mathrm{C}$, and $\mathrm{LAD}$ outlet temperature is $32.69^{\circ} \mathrm{C}$ 
Figure 7.35. Structural-thermal relationship in HH97 LCX for $68.85 \mathrm{ml} / \mathrm{min}$ guide extension flow rate. Note: proximal guide extension inlet temperature is $19.2^{\circ} \mathrm{C}$, and $\mathrm{LCX}$ outlet temperature is $34.55^{\circ} \mathrm{C}$.

Figure 7.36. Structural-thermal relationship in HH102 LAD for $49.03 \mathrm{ml} / \mathrm{min}$ guide extension flow rate. Note proximal guide extension inlet temperature is $23.16^{\circ} \mathrm{C}$, and LAD outlet temperature is $33.1^{\circ} \mathrm{C}$

Figure 7.37. Structural-thermal relationship in HH102 LAD for $68.85 \mathrm{ml} / \mathrm{min}$ guide extension flow rate. Note: proximal guide extension inlet temperature is $19.2^{\circ} \mathrm{C}$, and LAD outlet temperature is $30.47^{\circ} \mathrm{C}$

Figure 7.38. Structural-thermal relationship in HH102 LCX for $49.03 \mathrm{ml} / \mathrm{min}$ guide extension flow rate. Note: proximal guide extension inlet temperature is $23.16^{\circ} \mathrm{C}$, and $\mathrm{LCX}$ outlet temperature is $34.34^{\circ} \mathrm{C}$

Figure 7.39. Structural-thermal relationship in HH102 LCX for $68.85 \mathrm{ml} / \mathrm{min}$ guide extension flow rate. Note: proximal guide extension inlet temperature is $19.2^{\circ} \mathrm{C}$, and LCX outlet temperature is $31.54^{\circ} \mathrm{C}$

Figure 7.40. Temperature vs time plot for the proximal, mid, and distal infarct temperature probes during the infusion of chilled saline at $20^{\circ} \mathrm{C}$ in the coronary arteries. Proximal intracoronary temperatures reached $30^{\circ} \mathrm{C}$ during the occlusion phase and increased to $\sim 33^{\circ} \mathrm{C}$ for the duration of reperfusion prior to reaching body temperature at the end of the infusion

Figure 7.41. Temperature vs time display for intracoronary blood temperatures using TH therapy during PCI. Intracoronary blood temperatures were targeted consistently maintained around $33^{\circ} \mathrm{C}$ for the duration of the procedure. $\mathrm{TH}$ temperatures were maintained proximal to the occlusion in the coronary arteries for about 1 min and 15 seconds

Figure 8.1. Average cooling potential depth in the LAD and LCX mother vessel. LAD average cooling potential was deeper in each mother vessel compared to the LCX. However, standard deviations were smaller in the LCX, especially for higher flow rates.

Figure 8.2. Linear regression models for each flow rate showing the relationship between the mother vessel CE and decreasing values of $\eta_{T C}$. The $R^{2}$ term was used to determine how well our linear model fit the data. High GuideLiner® flow rates resulted in a good fit model, while the low flow rate resulted in a bad fit model. Accuracy of the model increases as $R^{2}$ grows closer to 1 
Figure 8.3. The relationship between the number of diverting branches from the mother vessel and decreasing total network cooling effectiveness $\left(\eta_{\mathrm{TC}}\right.$ ) (from left to right). The linear fit trendline suggests that $\eta_{\mathrm{TC}}$ is likely to decrease as the number of diverting branches from the mother vessel increase.

Figure 8.4. The relationship between the number of outlets in the LAD and LCX artery network and decreasing total network cooling effectiveness $\left(\eta_{\mathrm{TC}}\right.$ ) (from left to right). The linear fit trendline suggests that $\eta_{\mathrm{TC}}$ decreases as the number of outlets increase.

Figure 8.5. Percent of LAD pathway (mother vessel) experiencing TH-ABT. Increasing SA:V ratios are from left to right for each flow rate. HH88 and HH102 resulted in $100 \%$ effective rates at 49.03 and $68.85 \mathrm{ml} / \mathrm{min}$ flow rates. Effective rates of $100 \%$ result in TH-ABT's $\left(<35^{\circ} \mathrm{C}\right)$ evident at the LAD outlets.

Figure 8.6. Percent of LCX pathway experiencing TH-ABT. Increasing SA:V ratios are from left to right for each flow rate. HH102 and HH97 resulted in 100\% effective rates at 49.03 and $68.85 \mathrm{ml} / \mathrm{min}$ flow rates in $\mathrm{HH} 102$ and 68.85 $\mathrm{ml} / \mathrm{min}$ in HH97. Effective rates of $100 \%$ result in TH-ABT's $\left(<35^{\circ} \mathrm{C}\right)$ evident at the LCX outlets.

Figure 8.7. Mother vessel outlet ABT data plots for each GuideLiner® flow rate vs decreasing $\eta_{\mathrm{TC}}$ values. Seven recommended heart cooling candidates are isolated in the dashed box which are segregated by TH-ABTs $<35^{\circ} \mathrm{C}$ (horizontal line) and $\eta_{\mathrm{TC}}>97.63 \%$ (vertical line). 
Table

\section{List of Tables}

Table 1.1. Comparison between current TH treatment methods. 16

Table 3.1. Characteristic CFD Boundary Conditions and Relationship to Navier-Stokes and Thermal Energy

Table 4.1. Dimensionless values for Reynolds and Prandtl numbers assuming 68.85 $\mathrm{mL} / \mathrm{min}$ inlet flow rate and $0.003 \mathrm{~m}$ inlet artery diameter

Table 4.2. Critical flowrates and locations in mock circulatory loop

Table 4.3. Make and sizes of thermocouples used to measure guide inlet, guide extension outlet, heat exchanger, and aorta temperatures.

Table 4.4. Make and model of flow meters, thermocouples, and pressure transducer used in mock circulatory loop system.

Table 4.5. Make and model of peristaltic pumps, glass aorta, and Tuohy Borst adapters employed in the mock circulatory loop

Table 4.6. Operational ranges for each thermocouple used in the mock loop system.....46

Table 4.7. Operational ranges for each instrument used to measure flow rate and pressure

Table 4.8. Thermocouple calibration ranges, calibration points \& accuracy, and calibration check method

Table 4.9. Coronary test calibration guide for the operational ranges for each instrument Continued.

Table 4.10. Calibrated infusate flowrates exiting the guide extension from peristaltic pump in the cooling console

Table 4.11. Make and model for each medical device used in the mock loop system....51

Table 4.12. Various temperatures recorded from infusate flow rates ranging from 9.08 $\mathrm{ml} / \mathrm{min}$ to $88.9 \mathrm{ml} / \mathrm{min}$ in the cooling console circuit.

Table 4.13. Standard deviation (SD) for flow rates, guide extension inlet temperature, and guide extension outlet temperature

Table 4.14. Data obtained for the infusate flow rates, guide extension outlet temperatures, and aorta temperatures using Eq. 4.1 to solve for cooling capacity

Table 5.1. Length of proximal LAD and LCX arteries before major bifurcation .60 
Table

\section{List of Tables (Continued)}

Table 5.2. Total length of all vessels in each LAD and LCX structure .........................62

Table 5.3. Overall surface area for each HH model.

Table 5.4. Surface Area to Volume ratios for each LAD and LCX model employed in the study

Table 6.1. Descriptive parameters for each medical device used in the mock loop system

Table 6.2. Physics conditions used for each CFD heart model in STAR-CCM+

Table 6.3. Boundary conditions and equations applied to each human heart model in STAR-CCM+. The inlet conditions represent the final three flow rates used in the CFD simulations

Table 6.4. Material properties for blood and distilled water entering the proximal LAD and LCX [146][147][148]

Table 6.5. Blood and distilled water mixed fluid properties at variable GuideLiner ${ }^{\circledR}$ flow rates

Table 6.6. Key properties and assumptions for each mixed fluid.

Table 6.7. Three LAD and LCX models chosen at random and their associated mesh quality and computational time using two discrete computing hardware.

Table 6.8. Cell count and corresponding work station for each LAD and LCX model executed

Table 6.9. Thermal fluid model testing matrix used to describe the catheter type, location, inlet conditions, and models used in this thesis work. Please see Appendix C for a more comprehensive version of the thermal fluid modeling matrix ......90

Table 7.1. Summary of TH cooling effectiveness for all $\mathrm{HH}$ patients using $29.2 \mathrm{ml} / \mathrm{min}$ and $68.85 \mathrm{ml} / \mathrm{min}$ GuideLiner ${ }^{\circledR}$ flowrates

Table 7.2. Comparison of patient specifications and medical history with patients in similar heart cooling studies

Table 8.1. GuideLiner® flow rates associated with TH-ABT's leaving the LAD and LCX outlets

Table 8.2. Good and bad heart cooling candidates as recommended assuming 68.85 $\mathrm{mL} / \mathrm{min}$ proximal GuideLiner ${ }^{\circledR}$ flow rate 


\section{List of Tables (Continued)}

Table

Table 8.3. Good and bad heart cooling candidates with their corresponding number of outlets and vessel branches stemming from the mother branch 


\section{Chapter 1}

\section{Introduction}

\section{Basic Physiology of the Human Heart}

The human heart is an organ made of muscle that is responsible for pumping blood to the rest of the body and to itself via the aorta [1][2]. The heart consists of two atria and two ventricle chambers (Fig. 1.1) where de-oxygenated blood enters the right atrium via the superior vena cava.

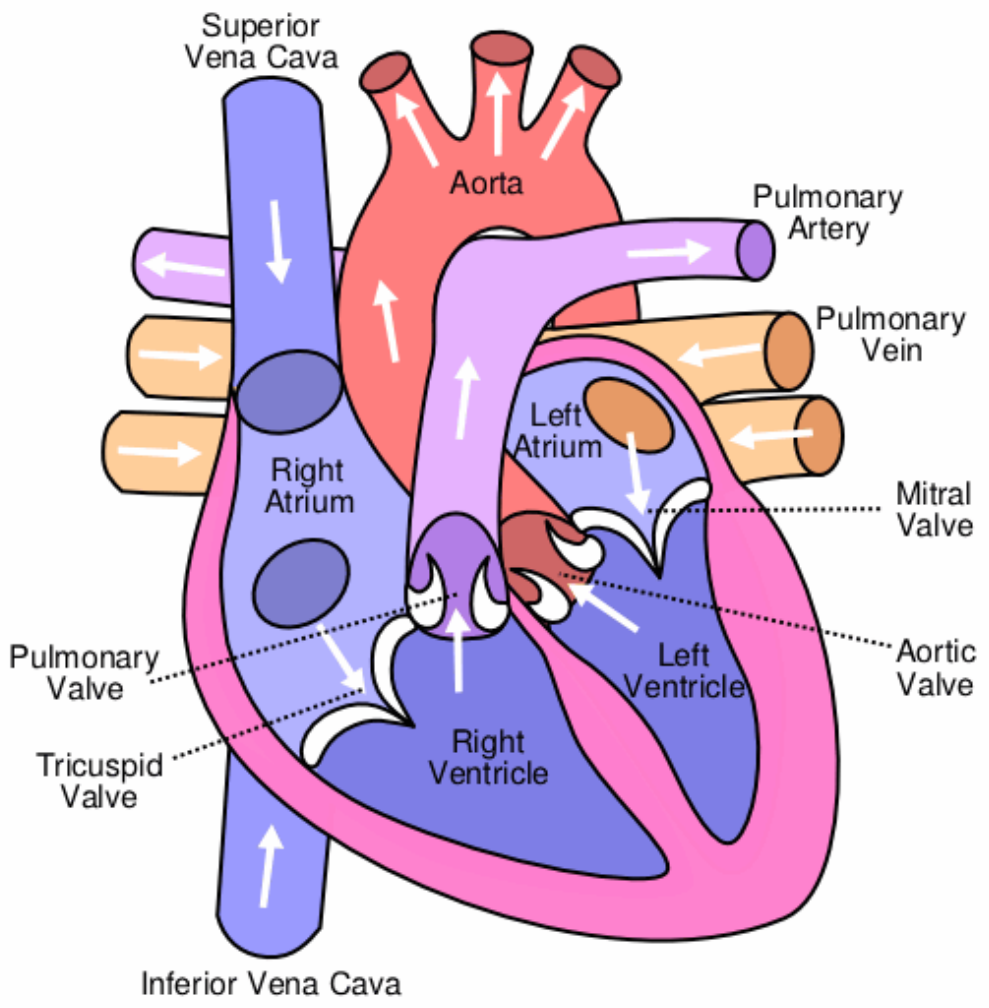

Figure 1.1. Basic cardiac circulation of the human heart. The right side of the heart supplies blood to pulmonary circulation, while the left side of the heart supplies blood to systemic circulation [3].

The right atrium contracts and blood is pumped into the right ventricle. When the left ventricle contracts, deoxygenated blood is pumped to the lungs through the 
pulmonary artery. In the lungs, blood releases carbon dioxide and loads up with oxygen. Oxygenated blood flows back into the left atrium, is pumped into the left ventricle, and then expelled into the aorta to sustain circulation.

The circulatory system consists of the heart, blood, and blood vessels, and circulates an average of 5 liters of blood throughout the human body [2]. Diastolic blood flow refers to the relaxation of the atrial and ventricle chambers. Systolic blood flow refers to the contraction of the atria and ventricle chambers. These contractions are initiated by action potentials from electrical excitatory impulses, however, the electrophysiology of the heart is beyond the scope of this work.

Vascular networks. After blood is pumped into the aorta, it enters the left and right coronary arteries to provide oxygen and nutrients to the myocardium (heart muscle) [2]. The left main coronary artery (LMCA), which originates from the proximal aortic arch, supplies blood to the left myocardium and bifurcates into the left anterior descending artery (LAD) and the left circumflex artery (LCX) [4]. The LAD supplies blood to the front of the heart, while the LCX circles the left myocardium and supplies blood to the back of the heart. The oblique LAD and LCX networks bifurcate into many daughter branches (Fig. 1.2) that span the surface of the heart. 


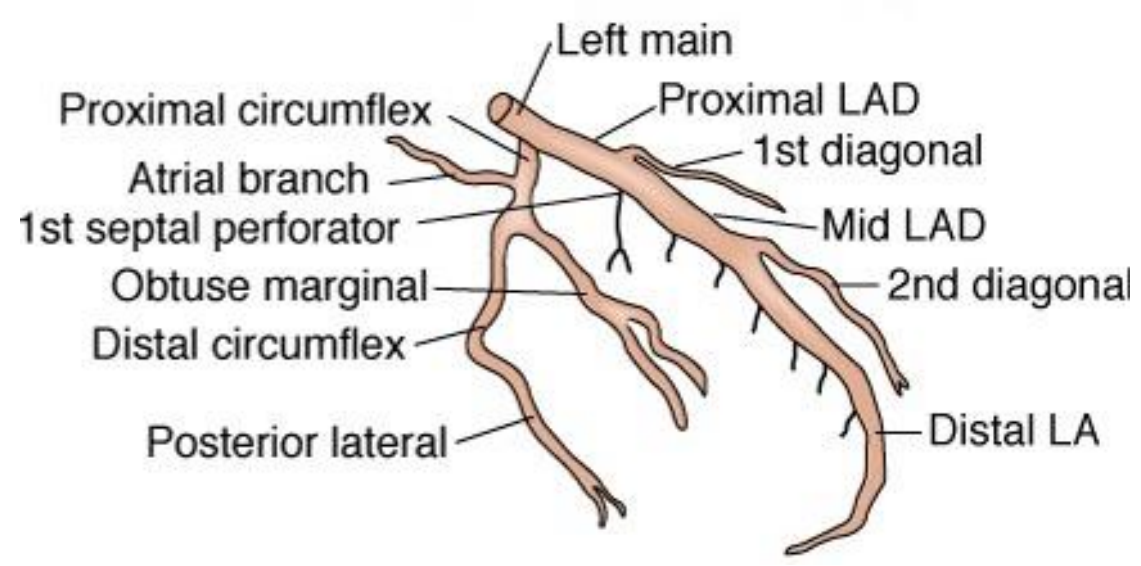

Figure 1.2. Image of the LMCA bifurcating into the LAD and LCX arteries that each branch into daughter vessels producing patient-specific artery networks. Each person has a unique coronary vascular network with arteries varying in length, diameter, and location of bifurcated branches [5]. This work will explore organ cooling along these vessels.

For the LAD, these branches consist of the $1^{\text {st }}$ septal perforator, $1^{\text {st }}$ and $2^{\text {nd }}$ diagonal, and the proximal, mid, and distal branches [5]. For the LCX, these branches consist of the atrial, obtuse marginal, and the posterior lateral branches. For this work, extensive research was performed to assess and determine appropriate LMCA geometries [6][7][8], native coronary flow rates [9]-[12], and catheter infusate flow rates for intracoronary procedures [13][14].

Coronary flow rates. Coronary flow rates have been measured in clinical settings using intracoronary doppler and ultrasound [11], phase contrast MRI video measurements [12], intracoronary frequency domain optical coherence tomography [15], and methods using doppler wires [16]. Using these methods, coronary flow rates ranged between 29.7 $\mathrm{ml} / \mathrm{min}$ and $107 \mathrm{ml} / \mathrm{min}$ in the LAD and $28.4 \mathrm{ml} / \mathrm{min}$ and $112 \mathrm{ml} / \mathrm{min}$ in the LCX $[9][10][11][12]$. Typical blood flow rates in the cardiac cycle (Fig 1.3) entering the left coronary artery network are pulsatile in nature and consists of the systole ejection phase and the diastole phase [17]. 


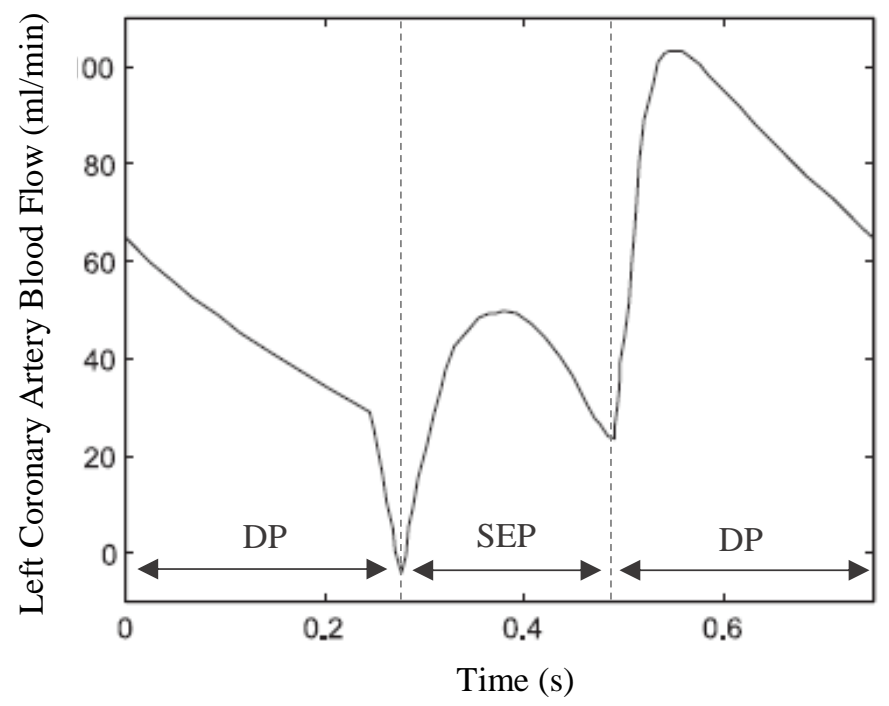

Figure 1.3. The cardiac cycle for blood flow entering the left coronary artery [17]. The typical cardiac cycle is pulsatile in nature and consists of the systole ejection phase (SEP) and the diastole phase (DP), which represents the time between $0.3 \mathrm{~s}$ to $0.5 \mathrm{~s}$ and $0.5 \mathrm{~s}$ to the beginning of the SEP cycle, respectively.

Variations between patients. Coronary artery geometry variations between patients affect nearly $5.6 \%$ of Americans, and include varying bifurcation patterns, vessel lengths and diameters, and dominant/co-dominant LAD and LCX networks [18]. Branching patterns in LMCA networks between patients include high variations of bifurcations and trifurcations, and if misdiagnosed, could cause difficulties in catheterization surgeries [19].

Coronary artery anomalies are defects in the geometry of the coronary arteries and are linked to an abnormal size or varying origin location from the aortic arch. These coronary artery anomalies between patients vary between $0.2 \%$ to $8.4 \%$ [20], and have been associated with congenital heart disease and approximately $15 \%$ of young athletes that experience sudden death [20][21]. Determining geometry patterns and variations in the coronary arteries is important for physicians to competently treat and diagnose cardiovascular disease. 


\section{Acute Myocardial Infarction (AMI)}

With the geometry and blood flow described, this research focuses on a common problem: acute myocardial infarction. Coagulation of inflammatory cells and calcium deposits may form on the inner walls of the coronary arteries, known as atherosclerotic plaque [22]. This plaque could rupture and form a clot (thrombus) to reduce blood flow or could form a complete blockage in the coronary arteries. This phenomenon can lead to insufficient nutrients and oxygen in the myocardium and is known as myocardial ischemia. If left untreated, the ischemia region can cause irreversible damage to the myocardium. This condition is known as acute myocardial infarction (AMI) [23].

Acute Myocardial Infarction (AMI) occurs when metabolically insufficient blood flows to the myocardium, resulting in cell death (necrosis) [23][24]. AMI is a primary cause of worldwide death and disability [23][25]. In contrast, AMI incidences have significantly declined over the past decade, however, it is estimated that 720,000 Americans will experience a new AMI and 335,000 will have a recurrent event in 2018 [26].

Permanent damage to the myocardium can result from AMI. While unresponsive to contractile function, fibrous scar tissue (infarct area) distal to a blocked artery (occlusion site) can form (Fig. 1.4) and cause impairment to left ventricular (LV) contraction, resulting in progressive chronic heart failure [23][24][13]. 


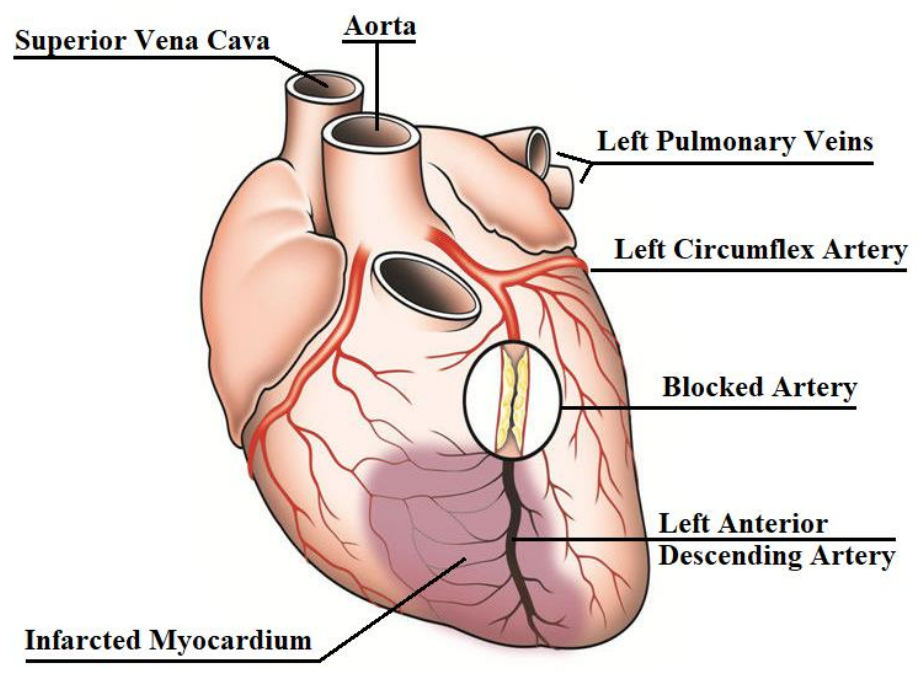

Figure 1.4. Schematic of left anterior descending artery occluded with plaque build-up. Purple area distal to occluded artery represents myocardial area at risk of infarction [27]. Therapeutic hypothermia is a promising treatment option that may reduce tissue damage and improve patient outcomes.

Additionally, patients who have experienced an AMI are at risk of a $10 \%$ fatality rate during hospitalization, and a $76 \%$ chance of developing chronic heart failure during the next 5 years [28][29][30][31].

\section{Ischemia Reperfusion Injury}

AMI is currently treated by methods of reperfusion, which aim to quickly restore blood flow to the oxygen-deprived myocardium. Reperfusion is the primary therapeutic goal for the treatment of AMI and has been shown to restrict the severity of infarction and even reduce mortality [24][25][26]. Paradoxically, clinical data has shown that significant myocardium damage can occur from blood reperfusion, accounting for up to 25 to $50 \%$ of the resulting myocardial infarct size [32][33]. This is known as ischemia reperfusion (IR) injury [24][33]. After the occlusion has been removed, the patient is at risk of both temporal and permanent myocardial injury. The former can span 48 hours to several days, while the later refers to permanent necrosis of myocardial cells after 
reperfusion [34][35]. There is currently no active therapy that prevents myocardial reperfusion injury in patients experiencing an AMI [28]. Most animal and human pilot studies have shown positive results, but a randomized controlled trial failed to replicate the outcomes [36].

To improve patient outcomes, new therapeutic strategies are being investigated to reduce permanent damage caused by IR injury. One therapeutic strategy is known as post-conditioning and is performed using a balloon catheter that opens an occluded artery and alters reperfusion mechanically. Moreover, this thesis work will focus on a novel cardioprotective strategy using therapeutic hypothermia [13][37][38][39][40].

Existing treatments. Several clinical trials have failed to show that drug therapy can reduce infarct size by limiting reperfusion injury [24][41][42]. Examples of failed clinical trials for reducing reperfusion injury and reducing myocardial salvage include the use of Adenosine [43] and inhaled nitric acid [36]. Future efforts that aim to prevent reperfusion injury or salvage ischemia myocardium should focus on non-pharmacological approaches as well [43]. An example of a widely used non-pharmacological intervention procedure to treat AMI is known as percutaneous transluminal coronary angioplasty (PTCA).

\section{Applied using percutaneous transluminal coronary angioplasty (PTCA). In} September of 1977, Andreas Grüntzig introduced and performed the first minimally invasive percutaneous transluminal coronary angioplasty (PTCA) in a patient. PTCA has since become the most widely used major intervention procedure to treat patients with angina and AMI [44][45]. PTCA offers a non-surgical treatment method for intervention in AMI, a primary source of mortality in western Europe and North America, and has recently improved its success rate since the introduction of stenting [46][47][48]. 
Timely use of revascularization procedures, or reperfusion to an organ or body part suffering ischemia, has shown to contribute to decreased mortality rates in patients who have undergone percutaneous coronary interventions (PCI) in ST-segment elevation myocardial infarctions (STEMI) [49]. As of 2009, median costs for PCI procedures (including intervention procedure and hospitalization) were $\$ 19,349$ [50].

Treatment to reestablish sufficient coronary blood flow after AMI using primary percutaneous coronary intervention (P-PCI) is performed using balloon angioplasty (Fig 1.5), with or without stenting [25].

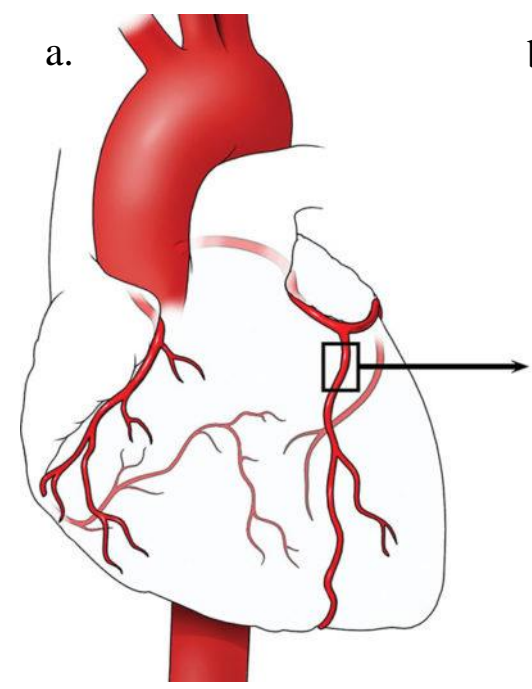

b.

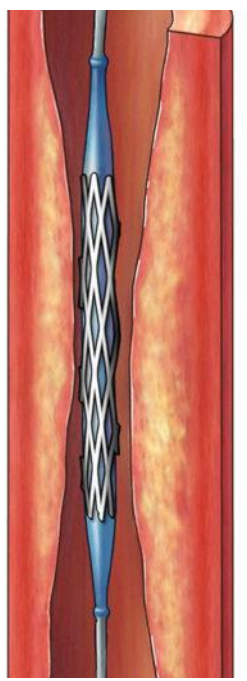

c.

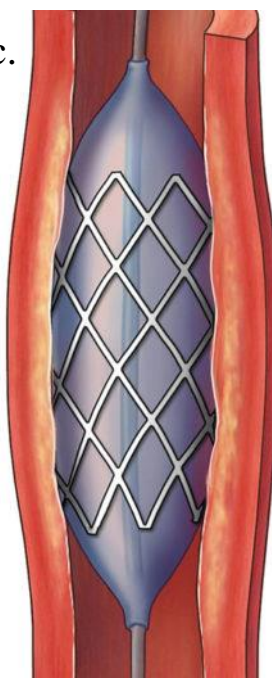

d.

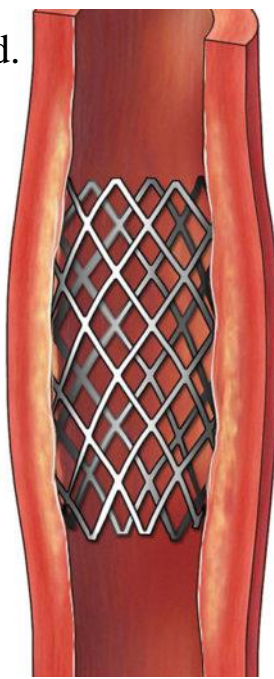

Figure 1.5. Primary percutaneous coronary intervention (P-PCI) performed using balloon angioplasty with stenting [51]. The occlusion is located in the artery network (a) and a guide is advanced through the femoral artery into the occluded vessel with the balloon and stent (b). The balloon is inflated (c) to expand the stent (d) and open the occluded vessel [25].

These devices for treating AMI are designed to be used in emergency cardiac catheterization laboratories. Stents used are either bare-metal stents (BMS) or drugeluting stents (DES) [52]. Of these, Cobalt chromium DES's exhibit the most favorable outcome to reduce AMI, stent thrombosis, and cardiac death rate [25]. 
Additionally, the use of post-conditioning (PC) is a moderately new revascularization procedure which is used to diminish IR injury by inducing transitory cycles of ischemia during the onset of reperfusion [53]. During this procedure, a PTCA balloon catheter is inserted into the femoral artery and advanced proximally to the occluded artery and is intermittently inflated and deflated per a predetermined algorithm. Although coupled with PTCA and PC procedures, external cooling blankets can result in side effects including increased infection risk, wound healing degradation, and patient discomfort from shivering due to unintentional overcooling [54][55].

Other treatments. Percutaneous transluminal coronary interventions (PTCI) have proven to be effective methods at improving heart function and diminishing the size of myocardial infarct [56]. However, clinical trials involving the use of PTCI with adjunctive therapies have failed to show positive results following myocardial infarction (MI) [57][58]. Other MI preventative treatments involve prescribing patients with antiplatelet $\mathrm{P}_{2} \mathrm{Y}_{12}$-receptor blockers prior to angioplasty procedures, which act to reduce vessel obstruction post PCI [56]. Still, clinical trials involving platelet inhibitors combined with PCI have failed to report positive results with PC [59][60]. MI outcomes will not be improved unless cardioprotective therapies surpass the effects of antiplatelet agents. Other treatment methods suggest the use of mild hypothermia coupled with sodium-hydrogen blockers that have helped to reduce infarction in rats when used with $\mathrm{P}^{2} \mathrm{Y}_{12}$ inhibitors [61]. A suggested solution to reducing MI is the development of an intervention that induces rapid cooling with platelet inhibitors during the time of reperfusion [56]. 


\section{Therapeutic Hypothermia}

Therapeutic Hypothermia (TH) aims to reduce normal tissue temperatures to improve patient outcomes [62][63][64].

History. Ancient Egyptian, Roman, and Greek physicians used hypothermia to treat humans with trauma and hemorrhage [65][66], and was even used in $400 \mathrm{BC}$ by Hippocrates who advised the use of snow and ice on wounded patients to reduce hemorrhage and bleeding [67]. TH was reintroduced in the 1940s to 1960s as a method to treat cardiac arrest patients [68][69][70], and was used as an effective method for organ transplantation, which was thereafter considered routine postoperative care [71][72]. Aside from the hospital, hypothermia has been used to salvage the brain in animals and humans succeeding cardiac arrest [63][64]. Hypothermia is recommended by the American Heart Association and the European Resuscitation Council to improve neurological recovery in comatose patients that have experienced a cardiac arrest [73].

Hypothermia can be categorized as mild, moderate, severe, or profound, with temperatures ranging between $32-35^{\circ} \mathrm{C}, 28-32^{\circ} \mathrm{C}, 20-28^{\circ} \mathrm{C}$, and $<20^{\circ} \mathrm{C}$, respectively [74]. TH is mostly attributed to mild hypothermia due to its hemodynamic stability in animals and humans. Moderate hypothermia could induce atrial fibrillation, while severe hypothermia could induce ventricular fibrillation [74][75][76]. To effectively reduce infarct size and safely diminish reperfusion injury, myocardial temperatures should be between $32-34^{\circ} \mathrm{C}[13][77][78]$.

Existing treatments. Current TH treatment methods consist of methods using external cooling blankets, percutaneous transluminal coronary angioplasty, and endovascular whole-body devices that require a separate insertion into the inferior vena cava. 
External cooling blankets. Non-invasive technologies are also under development. A non-invasive surface cooling apparatus called ThermoSuit has been tested on animals to treat early phase AMI via whole body cooling. However, along with similar noninvasive cooling blanket techniques, it remains a challenge to effectively cool patients at a cooling rate of $3.0^{\circ} \mathrm{C} /$ hour without causing adverse-effects such as frequent wound infections and disturbed shivering [79][80][81]. Moreover, US domestic hospitals have lethargically implemented the use of $\mathrm{TH}$ in their cases involving postcardiac arrest patients [82]. This is considered partially a result of TH unfamiliarity amongst physicians and the inaccuracy of external cooling using blankets and ice bags, which are usually inaccurate at maintaining TH temperatures [83].

Endovascular whole-body devices. Patients undergoing endovascular whole-body cooling interventions to treat AMI experience whole-body hypothermia at $33^{\circ} \mathrm{C}$ [13]. These procedures typically require a separate insertion in the femoral vein (Fig. 1.6) for the delivery of a cooling device to be positioned in the Inferior Vena Cava (IVC) [84]. 


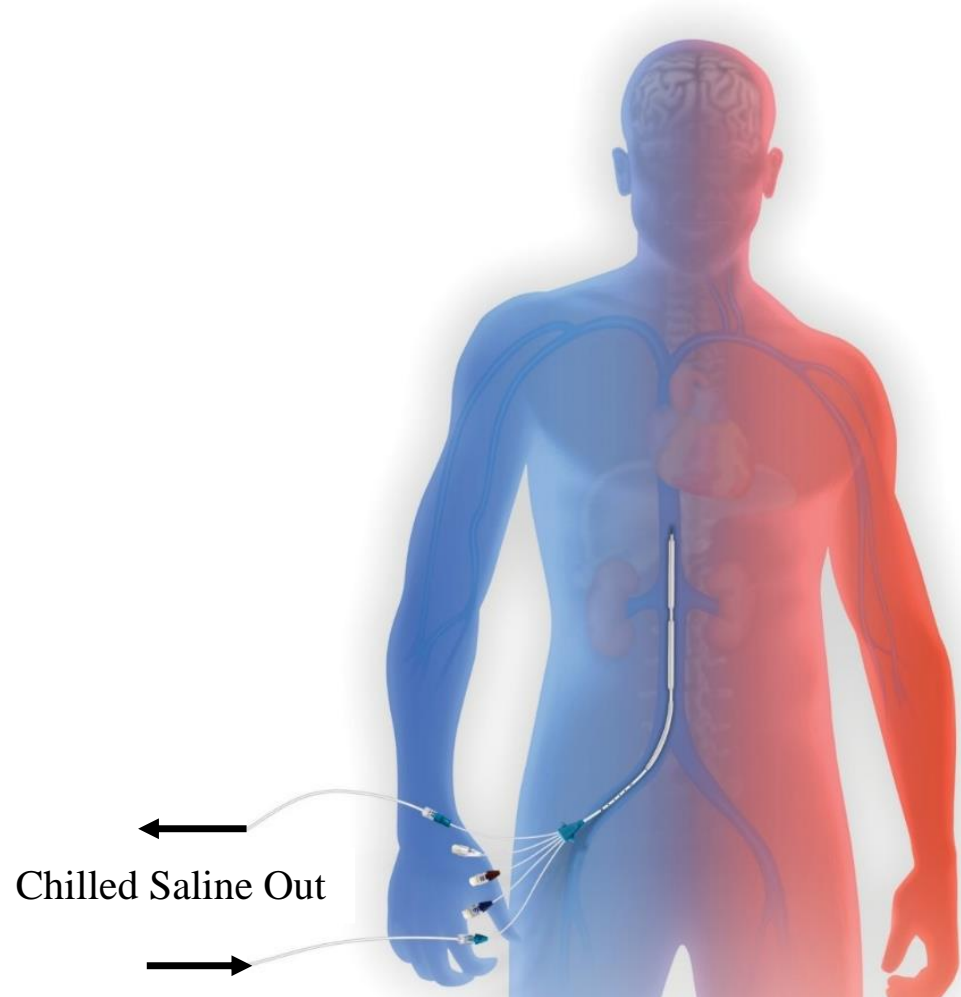

Chilled Saline In

Figure 1.6. Endovascular cooling device inserted in the femoral vein and positioned in the Inferior Vena Cava (IVC). Patients undergoing endovascular cooling interventions experience whole-body hypothermia at $33^{\circ} \mathrm{C}$ [13]. Endovascular cooling methods maintain target $\mathrm{TH}$ temperatures more efficiently than conventional external cooling but are unable to reach $\mathrm{TH}$ temperatures quicker [54].

Target temperatures of TH can be maintained more efficiently using endovascular cooling methods compared with conventional external cooling blankets [54]. Safety outcomes between endovascular and external cooling procedures are considered comparable, while endovascular cooling provides superior accuracy in sustaining TH target temperatures [85][86]. In comparison, endovascular whole-body cooling devices may maintain more accurate target temperatures but are unable to reach these temperatures quicker than conventional external cooling. 
Emerging treatments. Safe, rapid cooling of the heart is an important goal for physicians to effectively treat patients with AMI. Studies have shown that rapid hypothermia therapy $\left(<35^{\circ} \mathrm{C}\right)$ before reperfusion in patients with $\mathrm{AMI}$ can reduce infarct size by $37 \%$ [87][88][89][90]. This is known as Therapeutic Hypothermia (TH), which has shown feasibility for cardioprotection via methods of intravenous saline infusion and endovascular cooling [87][91]. The vast majority of AMI treatment methods involve whole-body cooling interventions, rather than heart-specific TH cooling devices, which are currently not on the market [92].

Localized therapeutic hypothermia. Studies have demonstrated the use of minimally invasive cooling catheters (Fig. 1.7) designed to locally induce TH by continuously delivering cooled fluids into the infarcted artery [92].

a.

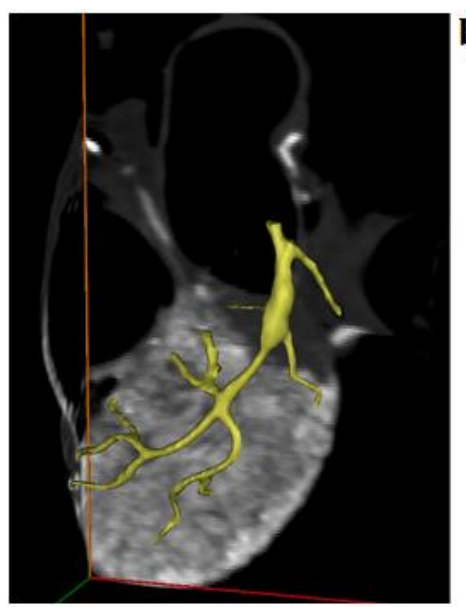

b.

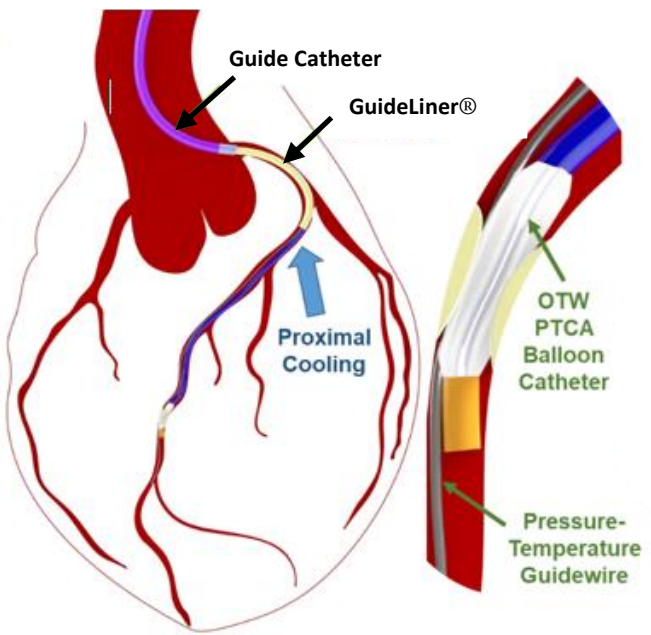

Figure 1.7. (a) 3D representation of the left anterior descending artery modeled in Mimics from DICOM HH63 (HH = Human Heart) from the Visible Heart Lab, University of Minnesota. (b) Schematic illustration of a standard guide catheter delivering cooled saline into left coronary artery via femoral artery access. 
Localized hypothermia has the potential to cool heart tissue rapidly following AMI, $3^{\circ} \mathrm{C}$ in 5 mins without the adverse-effects [93][94]. Nevertheless, the effectiveness of TH treatment for AMI, measured by myocardial salvage, will be impacted by the vascular structure with each patient (i.e. bifurcation patterns and vessel lengths and diameters). That vascular structure is akin to the internal design of an evaporator or a condenser, where each system, and analogously, each person is different.

Studies performed by Otterspoor [13] studied the impact of intracoronary hypothermia in patients with acute myocardial infarction. During the occlusion phase, 20 $\mathrm{ml} / \mathrm{min}$ infusate was delivered at room temperature $\left(22^{\circ} \mathrm{C}\right)$ proximal to the occlusion and the flow rate was adjusted to match a distal coronary artery temperature of $6^{\circ} \mathrm{Cbelow}$ body temperature. During the reperfusion phase (after occlusion removal), saline was infused at $16 \mathrm{ml} / \mathrm{min}$ at $4{ }^{\circ} \mathrm{C}$. Out of the ten patients enrolled in the study, six patients had occlusions in their LAD, one had an occlusion in the LCX, and three had an occlusion in the RCA.

Another study employed 73 patients, where 53 experienced stable angina (SA) and 20 had signs of ST-elevation myocardial infarction (STEMI) [95]. Guide catheter infusions delivered saline at room temperature at 10-30 $\mathrm{ml} / \mathrm{min}$ flow rates in the LAD and LCX arteries. The infusion catheter tip was fixed proximal to the occlusion and infusate was delivered at $-5^{\circ} \mathrm{C}$ below body temperature in the distal arteries.

In a study performed by McGarvey [96], ten patients experiencing STEMI endured primary angioplasty (PPCI) by receiving intracoronary infusate at room temperature through a guide catheter. Average intracoronary temperatures were reduced to $3.4^{\circ} \mathrm{C}$ below body temperature and infusate flowrates were delivered between 60 $\mathrm{ml} / \mathrm{min}$ and $100 \mathrm{ml} / \mathrm{min}$ proximal to the occlusion. A predetermined temperature of $30^{\circ} \mathrm{C}$ 
was set as a threshold to avoid the risk of hypothermia complications during infusate perfusions. Out of the ten patients included in the study, three occlusions were evident in the proximal LAD, two in the Mid-LAD, one in the LCX, and four in the RCA [96]. Advantages of localized cooling. In vivo trials reduced areas of heart tissue by $3^{\circ} \mathrm{C}$ in 5 mins using $30 \mathrm{~W}$ of cooling [92]. Rapid, localized cooling of heart tissue generally does not impact core body temperatures [94], and provides approximately ten times the cooling rate to that of external cooling devices, offering $\sim 1^{\circ} \mathrm{C}$ per minute compared to $0.1^{\circ} \mathrm{C}$ per minute [97].

A significant advantage to organ specific cooling is its ability to leverage the reduced thermal inertia, which results in rapid cooling of the myocardium. Since the product of mass and specific heat yield cooling capacity, it will be much quicker to cool a portion of a 310 gram heart than an entire 62 kilogram patient. Thus, cooling rates are significantly greater using localized cooling methods, which is a preferred method to use following an ischemia event [13][98][96]. Additionally, localized TH may serve as an effective co-therapy for angioplasty procedures.

Disadvantages of localized cooling. Localized organ cooling is a relatively new therapy and requires a substantial amount of research to assess its impact on damage caused to heart muscles across a broad spectrum of patient-specific vascular structures. Large clinical trials are needed to replicate localized cooling results from isolated pig heart studies [13] and small trials treating patients with STEMI [96]. There are major differences between localized cooling results from artificial occlusions in pig hearts and AMI in humans [99]. Lastly, optimal TH intracoronary temperatures have not been established for effectively reducing ischemia reperfusion injury [74][100]. Table 1.1 provides a comparison between current TH methods. 
Table 1.1

Comparison between current TH treatment methods.

\begin{tabular}{|c|c|c|c|c|c|}
\hline & $\begin{array}{l}\text { TH } \\
\text { Cooling } \\
\text { Rate }\end{array}$ & Method & $\begin{array}{l}\text { Hypothermia } \\
\text { Location }\end{array}$ & ASTT* & $\begin{array}{l}\text { Adverse- } \\
\text { Effects }\end{array}$ \\
\hline $\begin{array}{l}\text { External } \\
\text { Cooling } \\
\text { Blankets }\end{array}$ & $3^{\circ} \mathrm{C} / \mathrm{hr}$ & $\begin{array}{c}\text { External } \\
\text { Cooling } \\
\text { Pads }\end{array}$ & Whole-body & Effective & \multirow{2}{*}{$\begin{array}{c}\text { Frequent wound } \\
\text { infections \& } \\
\text { disturbed } \\
\text { shivering }\end{array}$} \\
\hline $\begin{array}{c}\text { Endovascular } \\
\text { Whole-Body } \\
\text { Devices }\end{array}$ & $3^{\circ} \mathrm{C} / \mathrm{hr}$ & $\begin{array}{c}\text { IVC* } \\
\text { Cooling }\end{array}$ & Whole-body & $\begin{array}{c}\text { More } \\
\text { Effective }\end{array}$ & \\
\hline $\begin{array}{l}\text { Localized } \\
\text { Cooling }\end{array}$ & $3^{\circ} \mathrm{C} / 5 \mathrm{~min}$ & $\begin{array}{l}\text { LMCA* } \\
\text { Cooling }\end{array}$ & Heart specific & $\begin{array}{c}\text { Most } \\
\text { Effective }\end{array}$ & $\begin{array}{c}\text { Substantial } \\
\text { research needed }\end{array}$ \\
\hline
\end{tabular}

Note. $*$ IVC $=$ Inferior Vena Cava, $*$ LMCA $=$ Left Main Coronary Artery, $*$ ASTT $=$ Accuracy at Sustaining Target Temperatures.

What others have done to examine the biophysics of heart cooling. Studies involving the biophysics of heart cooling remain elusive, specifically, the biophysics of coronary artery cooling following MI. Experimental studies have explored myocardial protection techniques using computer simulations to examine topical cardiac cooling [101], as well as conjugate heat transfer by computationally pumping cold liquid through the major cardiac chambers [102].

The majority of computational cardiac biophysics research targeted towards the coronary arteries have focused primarily on computing and visualizing hemodynamics (i.e. coronary blood shear, pressure, and velocity) [4][103][104][105], coronary artery fractional flow reserve (FFR) [106], and lumped parameter modeling [75][107]. A lumped parameter model is a mathematical model that describes the physiological constraints of a system using systematic mathematical calculations [108]. To our understanding, there is no explicit literature analyzing the biophysics of localized cooling in patient-specific coronary arteries. 
What is not well understood? The structural properties of the heart and its impact on thermal fluid behavior are poorly understood [92], [94], [109], [110]. A study performed by Otterspoor, 2018 involved the use of lumped parameter modelling to mathematically predict myocardial temperatures from intracoronary arterial temperature. However, higher fidelity and more complex calculations using computational fluid dynamics (CFD) modelling were not used, which could provide clinically significant physics-based models representing patient-specific vascular structures. CFD modelling methods are ideal tools to help determine the preferred flows and temperatures of intravascular GuideLiner® infusions for patient-specific procedures [13].

\section{Problem Statement}

Significance of work. The link between thermal fluid behavior and the structural geometry of the coronary arteries has not been sufficiently studied [95], [111]-[113]. Thermal fluid models of heart cooling, as well as models that predict arterial blood and myocardial tissue temperatures in patient-specific hearts do not currently exist [13].

Objectives of study. In this study, patient specific LAD and LCX artery models are used to help determine the impact of thermal fluid behavior from $\mathrm{TH}$ treatment methods [13]. A new thermal modeling approach is introduced that could provide a better understanding of the biophysics of AMI during $\mathrm{TH}$, as well as provide insight to guide more efficient TH device designs and operation parameters [92].

We aim to gain insight in determining distinguishable temperature behavior between six patient-specific LAD and LCX arteries by comparing temperature distributions of different coronary artery geometries and surface area variations. We also seek to reveal the effect of catheter flow rate on patient-specific temperature distributions in each LAD and LCX artery vasculature. The evaluation metric for comparison between 
models will be the measured distance of TH fluid temperatures along the fluid pathway. Success will be the result of TH fluid temperatures exiting the artery outlets.

The objective of this study is to create thermal fluid models of TH in six patientspecific human LMCA's predicting arterial blood temperatures for a variety of hearts and catheter infusion flow rates in the proximal LAD and LCX vasculature. CFD models can help determine and qualitatively analyze temperature distributions in the LMCA's. They can shed light on TH device design and operation to optimize patient outcomes following AMI. 


\section{Chapter 2}

\section{Biophysics Background}

The following chapter provides the biophysics background and describes the fundamental governing equations required to solve fluid mechanics and heat transfer problems in a human heart vascular network. This chapter will discuss the governing equations used in our computational models including conservation of linear momentum and conservation of thermal energy. The end of the chapter will describe an equation for solving dynamic viscosity as a function of shear rate, hematocrit, and temperature.

\section{Laminar Flow: Conservation of Linear Momentum}

Governing equations. The Navier-Stokes equations describe the conservation of linear momentum for fluid flow in three-dimensional space, and are represented in Cartesian coordinates as the following [114]:

$$
\begin{gathered}
u \frac{\partial u}{\partial x}+v \frac{\partial u}{\partial y}+w \frac{\partial u}{\partial z}=-\frac{1}{\rho} \frac{\partial p}{\partial x}+v \frac{\partial^{2} u}{\partial x^{2}}+v \frac{\partial^{2} u}{\partial y^{2}}+v \frac{\partial^{2} u}{\partial z^{2}} \\
u \frac{\partial v}{\partial x}+v \frac{\partial v}{\partial y}+w \frac{\partial v}{\partial z}=-\frac{1}{\rho} \frac{\partial p}{\partial y}+v \frac{\partial^{2} v}{\partial x^{2}}+v \frac{\partial^{2} v}{\partial y^{2}}+v \frac{\partial^{2} v}{\partial z^{2}} \\
u \frac{\partial w}{\partial x}+v \frac{\partial w}{\partial y}+w \frac{\partial w}{\partial z}=-\frac{1}{\rho} \frac{\partial p}{\partial z}+v \frac{\partial^{2} w}{\partial x^{2}}+v \frac{\partial^{2} w}{\partial y^{2}}+v \frac{\partial^{2} w}{\partial z^{2}}
\end{gathered}
$$

where $v$ represents the kinematic viscosity having the form $\frac{\mu}{\rho}$, where $\mu$ is the dynamic viscosity as a function of temperature and shear rate.

Blood will be considered as an incompressible, Newtonian fluid, since the viscosity is dependent on shear rate [139][140], with a constant (or average) shear rate assumption. As a result, viscosity will only vary with temperature, providing there is coupling between the energy and Navier-Stokes equations. This concept will be described in further detail later in this chapter. 
Pulsatile flow is a natural phenomenon that occurs in physiological systems, such as the human respiratory and circulatory system [17]. However, the heat transfer coefficient for steady and pulsatile flow are equivalent [115][116], and studies have shown that pulsating effects have no impact on heat transfer in blood flow [117][118]. Therefore, pulsating flow effects were disregarded and steady state simulations were used to solve for ABT distributions that would typically reflect pulsatile flow conditions.

Steady state flow occurs when the fluid properties, such as temperature and mass flow rate, are unchanging over time for any single point in the system. Steady state simulations are also computationally cost efficient since they typically complete using significantly less CPU time than unsteady state simulations. For this reason, steady state CFD simulations are typically used as a starting point to verify that physics conditions and fluid properties are accurately solved [119]. Therefore, for this research, steady state, 3dimensional laminar flow will be assumed.

Governed by the laminar Navier-Stokes equations of conservation of linear momentum (2.4a), and the continuity equation of conservation of mass (2.5), the equations employed in this study can be written in the following form [120]:

$$
\begin{gathered}
\rho \frac{d V}{d t}=\rho g-\nabla p+\mu \cdot \nabla^{2} V \\
\nabla \cdot V=0
\end{gathered}
$$

where $V$ is the fluid velocity, $\nabla$ denotes the operator, $\rho$ is the density of the fluid, $g$ is gravity, $p$ is the pressure of the fluid, and $\mu$ is the dynamic viscosity of the fluid. Equation 2.4a is the more frequently seen version of the Navier-Stokes equations. However, for this work, we will use a non-constant viscosity encapsulated by the more general form of Navier-Stokes:

$$
\rho \frac{d V}{d t}=\rho g-\nabla p+\nabla \cdot \mu(\nabla V)
$$


where the viscous stresses inside of the divergence operator come from $\mu$ times the velocity gradient. Since the Navier-Stokes equations indicate a force per unit volume, the viscous force (per unit volume) is resultant of viscous stress (per unit area), where area forces are integrated to volume forces which are reconciled through the divergence theorem [121]. The viscous terms are then split between equations $2.4 \mathrm{a}$ and $2.4 \mathrm{~b}$, resulting in the external divergence operator as seen in Eq. 2.4b [115].

In cylindrical coordinates, the gradient operator $\nabla$ represents the following in three dimensions:

$$
\nabla=\frac{\partial}{\partial r} \hat{r}+\frac{1}{r} \frac{\partial}{\partial \theta} \hat{\theta}+\frac{\partial}{\partial z} \hat{z}
$$

where $\hat{r}, \hat{\theta}$, and $\hat{z}$ are unit vectors associated with their corresponding $\mathrm{r}, \theta$, and $\mathrm{z}$ directions in cylindrical coordinates. Equations 2.1 to 2.5 represent the foundation of all computational fluid dynamics (CFD) modeling. Chapter 8 describes the innovative approach of this work and limitations of the before mentioned assumptions.

\section{Laminar Flow: Conservation of Thermal Energy with constant wall temperatures}

Governing equations. The thermal energy equation for an incompressible fluid that satisfies the continuity equation (Eq. 2.5) can be described as a 3-dimensional problem with no kinetic energy or time derivative of pressure $\frac{\partial p}{\partial t}$, having the form [114]:

$$
\rho C_{p}\left[u \frac{\partial T}{\partial x}+v \frac{\partial T}{\partial y}+w \frac{\partial T}{\partial z}\right]=k\left[\frac{\partial^{2} T}{\partial x^{2}}\right]+k\left[\frac{\partial^{2} T}{\partial y^{2}}\right]+k\left[\frac{\partial^{2} T}{\partial z^{2}}\right]
$$

where the specific heat, $C_{p}$, and thermal conductivity, $\mathrm{k}$, are assumed constant, $\mathrm{T}$ is the absolute temperature, and the terms on the right side of the equation represent the rate of thermal diffusion.

Eq. 2.7 is coupled with Eq. 2.4 because the viscosity, $\mu(T)$ is a function of temperature, where T is determined from Eq. 2.7. Therefore, Eq. 2.4 and Eq. 2.7 are 
coupled to solve for each iterative temperature and viscosity along the fluid flow path, respectively. These values are then used as inputs to solve for fluid flow in the NavierStokes equations (Eq. 2.1 - 2.3). Appendix A contains detailed derivations of the fully developed velocity profile, energy balance, and the temperature dependent heat source equation in cylindrical coordinates. Additional information regarding CFD modeling methods will be discussed in greater detail in Chapter 3.

\section{Post-Processing}

The average bulk temperature (ABT) in fluid flow is the mean temperature of the fluid's cross section [115]. For complicated LMCA networks, the ABT changes in the direction of flow and is a critical post-processing component for determining LMCA outlet temperatures. The rate of energy flowing through a cross section is considered equivalent to the energy rate travelling through said cross section with a constant fluid temperature [115]. Therefore, the flow rate of thermal energy of the fluid can be expressed as the following:

$$
\dot{E}_{\text {fluid }}=\dot{m} c_{p} T_{m}=\int_{A_{c}} \rho u c_{p} T(r, x) d A_{c}
$$

where $A_{c}$ is the cross-sectional area, $T_{m}$ is the mean temperature, $\rho u$ is the mass flux, where $u$ has $(r, x)$ dependence, and $c_{p} T(r, x)$ is the internal energy per unit mass. Thus, assuming a circular boundary, solving for mean temperature yields:

$$
T_{m}=\frac{\int \rho u c_{p} T(r, x) d A_{c}}{\dot{m} c_{p}}=\frac{2}{u_{m} r_{o}^{2}} \int_{0}^{r_{o}} u T(r, x) r d r
$$

where $u_{m}$ is the mean velocity, and $r_{o}$ is the radius of the cross section of interest.

\section{Properties}

When non-isothermal fluids experience a temperature change, the material properties, such as density and viscosity, are also subject to change [122]. Determining the material properties of a fluid is a critical step in solving fluid dynamics problems. The 
physics of fluid dynamics simulations hinge upon material properties that accurately reflect the properties of the problem being solved.

Dynamic viscosity. Dynamic viscosity $(\mu)$ for the mixed blood and distilled water in the LAD and LCX arteries was modelled using an equation derived by Cheung et al. 2000 [123]. The model was used in our study to calculate the viscosity of the mixed fluid between 0 and $40{ }^{\circ} \mathrm{C}$. The Dynamic viscosity is a function of shear rate, hematocrit, and temperature, and can be expressed as:

$$
\mu\left(\dot{\Upsilon}, \text { Hct }, T_{m}\right)=\left(e^{\frac{\lambda}{\dot{\gamma}+\eta \cdot H c t}}\right)\left[\alpha+\frac{\phi}{\left(1+e^{\beta\left(T_{m}-\epsilon\right)}\right)}\right]
$$

where the shear rate $(\dot{\Upsilon})$, hematocrit value $(\mathrm{Hct})$, and mean temperature $\left(T_{m}\right)$ are independent variables and the dynamic viscosity is the dependent variable for this solution. The remaining coefficients, $\lambda, \eta, \alpha, \phi, \beta$, and $€$, represent predetermined values derived by Cheung et al. 2000 [123]. A total mixed Hematocrit value was determined for each of the catheter flow rates, and an average shear rate was calculated for each of the six LMCA models. Therefore, equation 2.11 is simplified to become:

$$
\mu\left(T_{m}\right)=C \cdot\left[\alpha+\frac{\phi}{1+f\left(T_{m}\right)}\right]
$$

where $C$ is a constant, and $f(T)$ is how equation 2.7 is coupled to the Navier-Stokes equations (2.1 to 2.3). The common Newtonian form of the Navier-Stokes equations (Eq. 2.4) is now extended to non-Newtonian fluids, which now requires the energy balance equation to solve for temperature.

The treatment of hematocrit and shear rate effectively prescribes a vasculaturespecific Newtonian flow for each LMCA simulation. Additionally, full non-Newtonian treatment would require significantly more complex analysis [124][125]. 


\section{Chapter 3}

\section{Computational Methods}

A number of computational methods were required to solve the thermal fluid modeling work described this thesis. The modeling workflow and CFD simulation process is described in detail.

\section{Materialise Mimics® Workflow}

Mimics (Materialise's Interactive Medical Image Control System) Innovation Suite 20 is one of Materialise's high-end processing tools used to create 3D models from 2D cross-sectional computed tomography (CT) or magnetic resonance imaging (MRI) medical image stacks [126].

\section{DICOM Image Sets to 3D Vasculature Creation}

The images employed in this study consist of image stacks known as DICOM (Digital Imaging and Communications in Medicine) image files [127]. DICOM images are typically attained from hospitals and/or doctors and are used to analyze specific organs of the body. DICOM defines the international standard for format use of medical images that satisfy the quality and requirements to transmit, store, and process clinical use imaging information [127]. For this study, the DICOM image sets consisted of CT angiography axial image stacks with an average slice thickness of $0.942 \mathrm{~mm} \pm 0.3$, a plane resolution of $512 \times 512$ pixels, and an average pixel resolution of $0.377 \mathrm{~mm} \pm$ 0.085. The source of where the DICOM images came from and rationale for selecting specific human heart models is discussed in Chapter 5.

DICOM files were imported into Mimics Innovation Suite 20 (Mimics®) as stacks of axial images in the XY plane (axial plane). Mimics® interpolates the image stacks to produce a 3D impression of the model (Fig. 3.1) by creating images in the $\mathrm{XZ}$ and YZ planes for the coronal and sagittal views, respectively. 

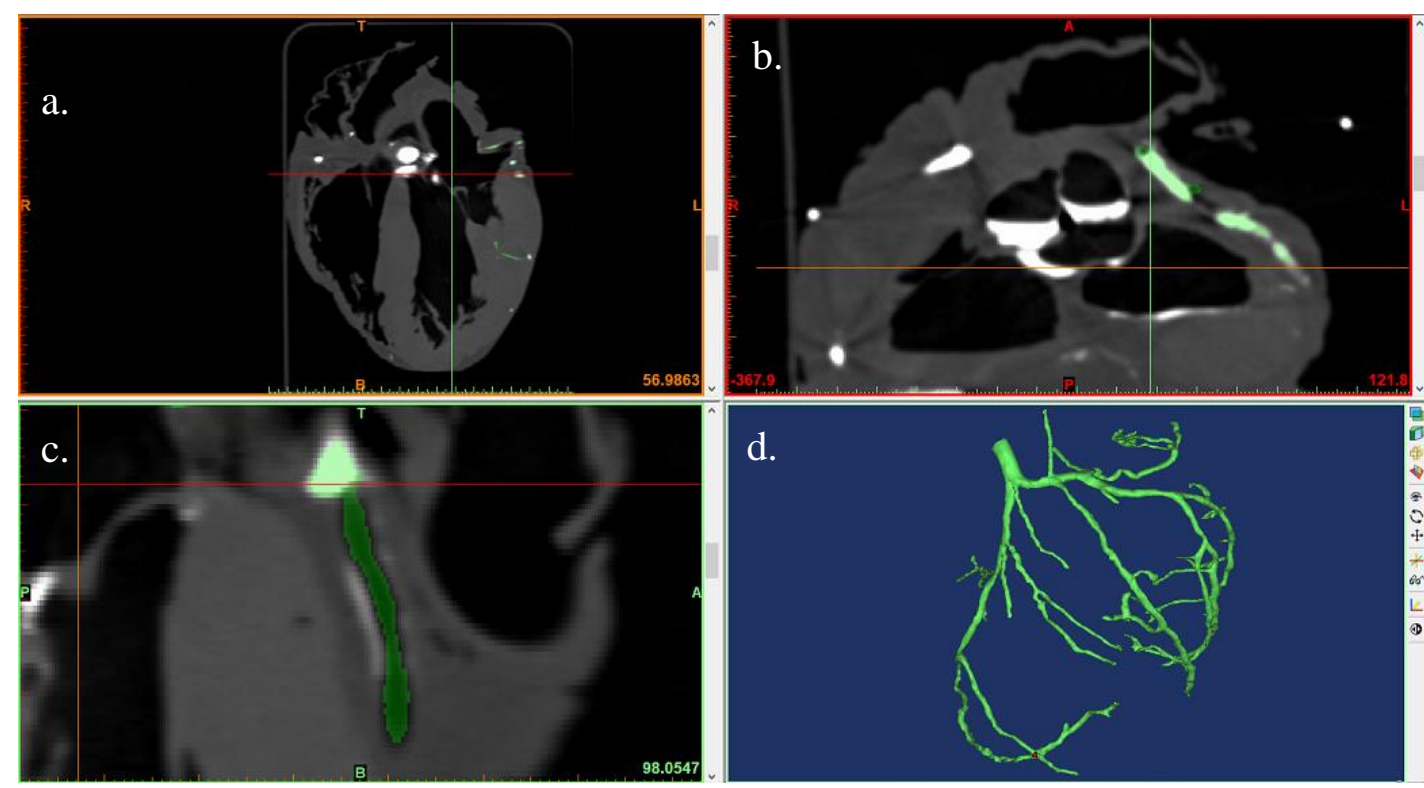

Figure 3.1. 3D volume rendering window in Mimics ${ }^{\circledR}$ of the LMCA segmented from 2D CT angiography image scans of HH55. The views in the window pane consists of the coronal (a), axial (b), sagittal (c), and the 3D rendering window (d). Green highlighted pixels (c) represent the segmented blood flow path of the artery network. The fully segmented 3D model (d) represents a patient-specific structure that can be exported in STL format and used in CFD simulations for thermal-fluid flow analysis.

Segmentation approach. CT scanners produce medical images that exhibit grayscale information. Mimics utilizes the grayscale information by enabling the user to highlight individual grayvalues, known as Hounsfield units, within each image [126]. Grayvalues are numbers that represent each pixel on an image that correlate to both the density and shade (black, white, or gray) of the pixel. Mimics groups together similar grayvalues so that an image can be segmented; a process used to convert anatomical image data sets to 3D models. During segmentation, the vasculature of interest for each pixel of the data set images was highlighted. This data was then translated from the segmented images to create a 3D model of the accentuated vasculature [126]. This method is known as thresholding and was used to create accurate 3D representations of the accentuated physiological LMCA models [126]. 
STL format. Mimics® models were exported in stereolithography (STL) format (Fig. 3.2, Step 3).
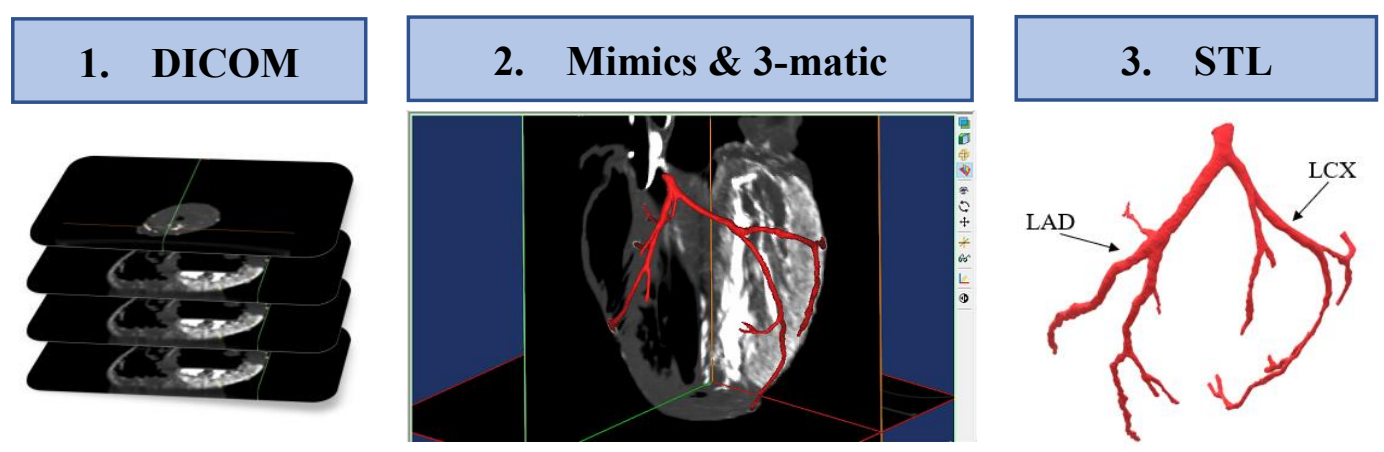

\section{SolidWorks}

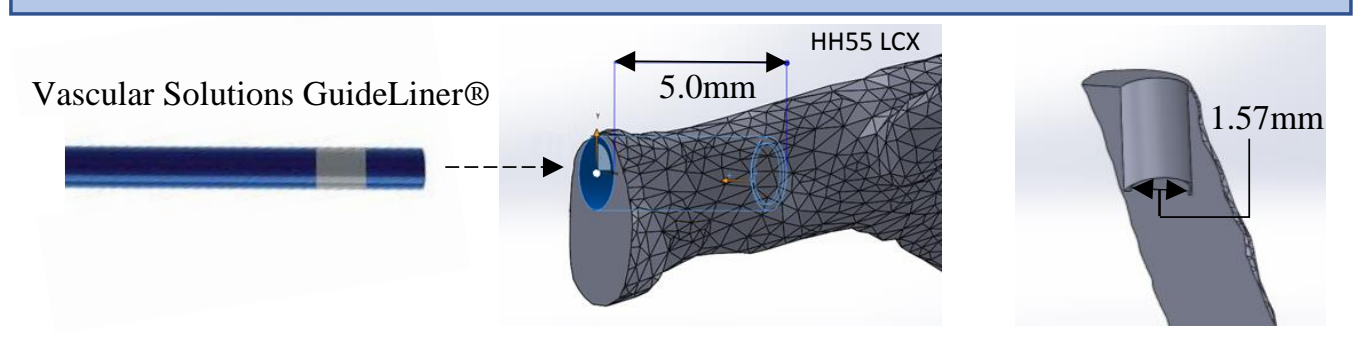

Figure 3.2. DICOM to SolidWorks editing workflow. DICOM images were attained from the Visible Heart Lab, University of Minnesota (Step 1) and imported into Mimics for 3D model segmentation and 3-matic smoothing (Step 2). The Mimics/3-matic models were exported in binary STL format (Step 3) and imported into SolidWorks for inlet/outlet trimming and creation of the proximal GuideLiner® insertion (Step 4).

STL files represent the surface geometry of 3D objects using a triangulated surface mesh without representing the color or texture of the object of interest. The surface geometry of STL files are encoded with triangular configurations such that there are no gaps or overlaps in the outer-surface portrayal, or shell, of the model's vasculature. This meshing structure allows for a simple, yet accurate representation to match most complex geometry structures. STL files are generated as compact binary versions of a model, which can be imported into 3D engineering programs for post processing [128] and computer aided design (CAD) manipulation [126]. 


\section{Materialise 3-Matic Model Smoothing}

The Mimics® STL files were exported into Materialise 3-matic, an extension of Materialise's engineering software tools that enable detailed design manipulation of STL files. This software was used to perform advanced editing and remeshing of the STL LMCA models, such as trimming sharp edges and filling holes in the mesh that were created during the segmentation process.

\section{SolidWorks Editing}

With an STL file formed by Mimics and the original DICOM data set, the binary STL files from the LAD and LCX heart models were imported into a 3D computer-aided design (CAD) modeling and editing tool, SolidWorks ${ }^{\circledR}$, where the inlet and outlets were orthogonally trimmed (Fig. 3.3). 


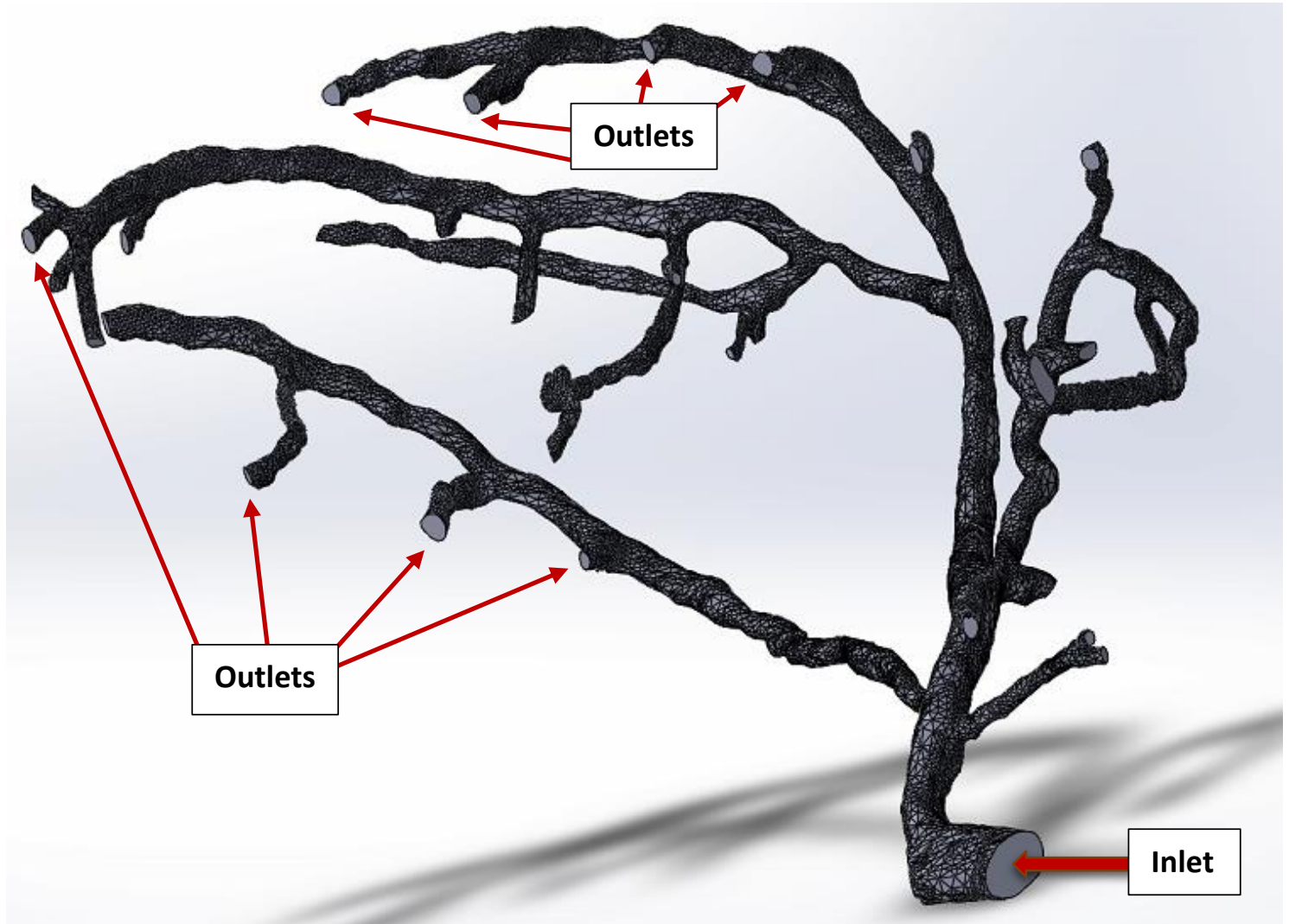

Figure 3.3. With an STL file formed by Mimics and the original DICOM data set, a 3D modeling and editing tool, SolidWorks ${ }^{\circledR}$ was used to orthogonally trim the inlet and outlets of each LAD and LCX artery model. The following HH55 LCX model contains a single inlet and 33 outlets, however, the number of outlets varied with each LAD and LCX model employed in this work.

When Mimics® STL files are exported into SolidWorks®, the geometry of the model slightly changes, which leads to an increased risk of surface repair and meshing issues. Each software tool that is used to refine and edit the model contributes to a possible surface meshing issue, which could cause simulation errors in computational modeling work. Therefore, these errors should be identified and remedied in each software tool. For example, holes in the surface mesh were identified in 3-Matic and filled to ensure no surface meshing errors carried over into STAR-CCM+. 


\section{Brief Description of Siemens STAR-CCM+ Workflow}

Finite volume method. The Finite Volume Method (FVM) is a computational method used to solve complex problems involving partial differential equations (PDE's). The FVM uses a discretization process that interprets a set of governing equations defined on a computational domain. The domain is discretized into a system of structured control volumes, or cells (Fig. 3.4) [114].

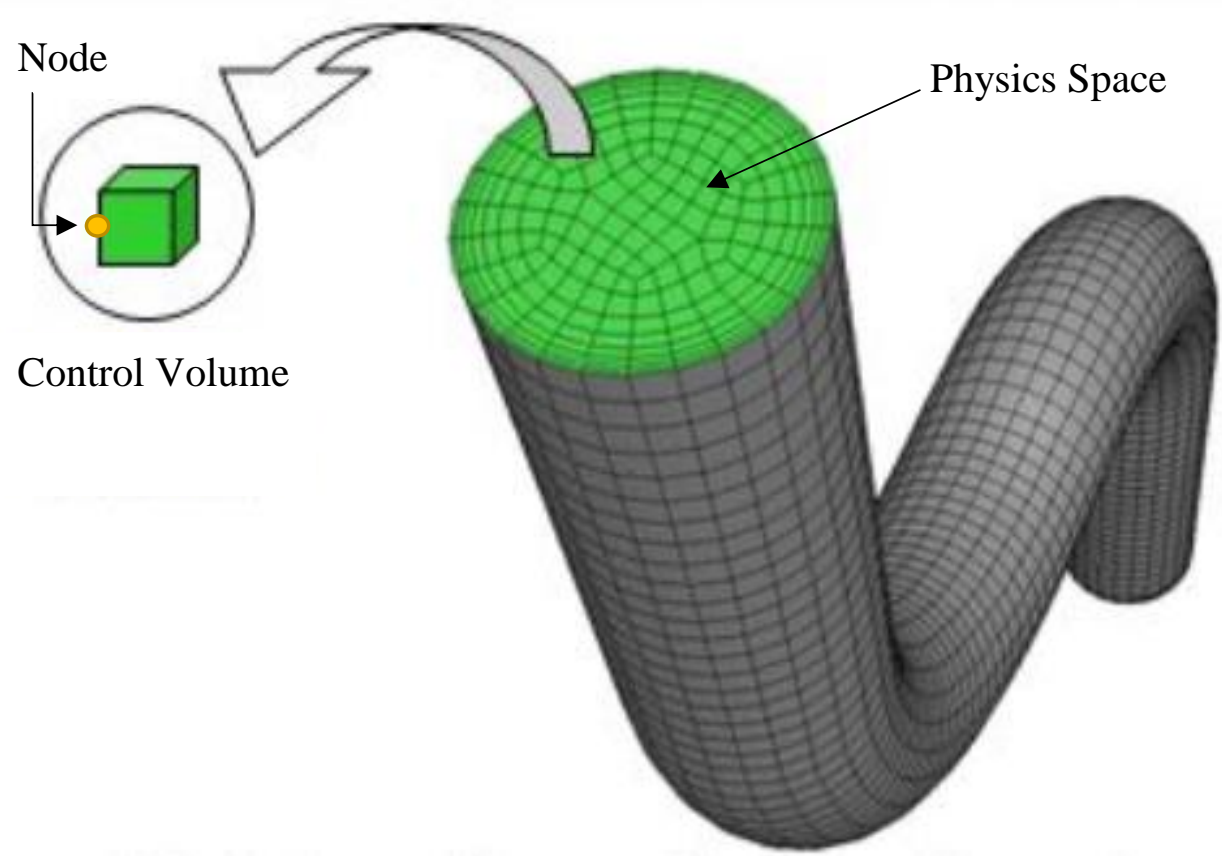

Figure 3.4 The physics space (1) in a simple bent tube is comprised of a grid that encompasses the computational domain. At each node (2), the governing equations in algebraic form are created. If we combine 1 and 2, a system of equations can be used in STAR-CCM+ to solve the flow field [129].

The FVM approximates a formation of ordinary differential equations (ODE's) that describe the computational mesh comprising of the minute control volumes (i.e. cells, elements) [114][130]. These equations employ iterative solvers that are able to compute numerical solutions in the computational domain space per unit time. A more 
accurate solution can be obtained by refining the mesh within the boundary conditions, which provides a more precise assessment of the ODE solvers in the physics domain.

FVM mathematics. Conservation laws from the Navier-Stokes equations are applied in integral form when solving FVM [131]. A succession of finite control volumes permeates the computational domain and are executed in the physical space using identical coordinate systems. To describe how FVM works mathematically, consider the incompressible continuity equation (Eq. 2.2) from Chapter 2 , where $\nabla \cdot v=0$.

Integrating Eq. 2.2 over a control volume for an incompressible fluid where $\rho$ is constant, yields:

$$
\int_{v_{i}} \nabla \cdot v d V=0
$$

where $v$ is the fluid velocity, $\mathrm{dV}$ is the volume element, and $v_{i}$ is the control volume integrated over the surface. Applying the divergence theorem, we obtain:

$$
\oint_{S_{i}} v \cdot \boldsymbol{n} d S=0
$$

where $\boldsymbol{n}$ is the unit normal to the volume element and dS is the surface area of the volume element. Thus, Eq. 3.2 represents a boundary integral typically used in FVM analysis describing the continuity for a control volume [132].

The governing equations described in Chapter 2 are discretized into algebraic equations that are solved across the computational domain at discrete locations to numerically represent fluid flow and heat transfer values. The mesh representing the computational domain is a three-dimensional discretization that should represent independence of the governing equations from additional mesh refinements.

Boundary conditions applied to FVM. To get a better understanding for how the Navier-Stokes (NS) and Thermal Energy equations work together to solve CFD problems, a general correlation to boundary conditions can be expected (Table 3.1). 
Table 3.1

Characteristic CFD Boundary Conditions and Relationship to Navier-Stokes and Thermal Energy.

\section{Boundary Condition Navier-Stokes Thermal Energy}

$\begin{array}{ccc}\text { In } & \dot{m} & T_{\text {in }} \\ \text { Out } & \mathrm{P}=0 & \frac{\delta T}{\delta s}=0 \\ \text { Wall } & \text { Velocity }=0 & T_{\text {wall }}=\text { constant }\end{array}$

The NS boundary condition entering our inlet region represents flow that is fixed to typical mean flows, while NS at each of our outlet regions represents $\mathrm{P}=0$, meaning there is no backpressure acting on our system at the outlets. NS for the walls signify a zero velocity no slip boundary condition represented by a fixed (stationary) wall. The Thermal Energy entering our inlet region represents physiological blood temperature at the artery inlet and chilled saline temperatures at the GuideLiner ${ }^{\circledR}$ inlet. Exact temperatures are listed in Chapter 4. Thermal Energy exiting our domain is built on our assumption of having sufficient vascular lengths such that the gradient along the vessels are negligible. Lastly, our Thermal Energy boundary condition acting at the walls represents a constant, isothermal physiological body temperature.

Since NS is especially useful in solving fluid dynamic simulations, while thermal energy is utilized to solve heat transfer phenomena, the two can be coupled together to denote distinct boundary conditions used in solving our CFD models. Although NS and Thermal Energy equations work together in CFD heat transfer simulations, they are contingent upon dynamic viscosity, $\mu$, and its associated temperature.

More complicated thermal boundaries could be envisioned in lieu of $T_{\text {wall }}=$ constant, however, at present, no work yet exists even for isothermal walls. Therefore, 
the effect of vascular branching and concentrated heat sources from close branches can be ignored.

Mesh convergence. A mesh convergence analysis is required to attain an optimum mesh. If the mesh contains large cells, the number of cells in the mesh would be minimal (Fig. 3.5) and the temperature would vary dramatically below the optimum number of cells.

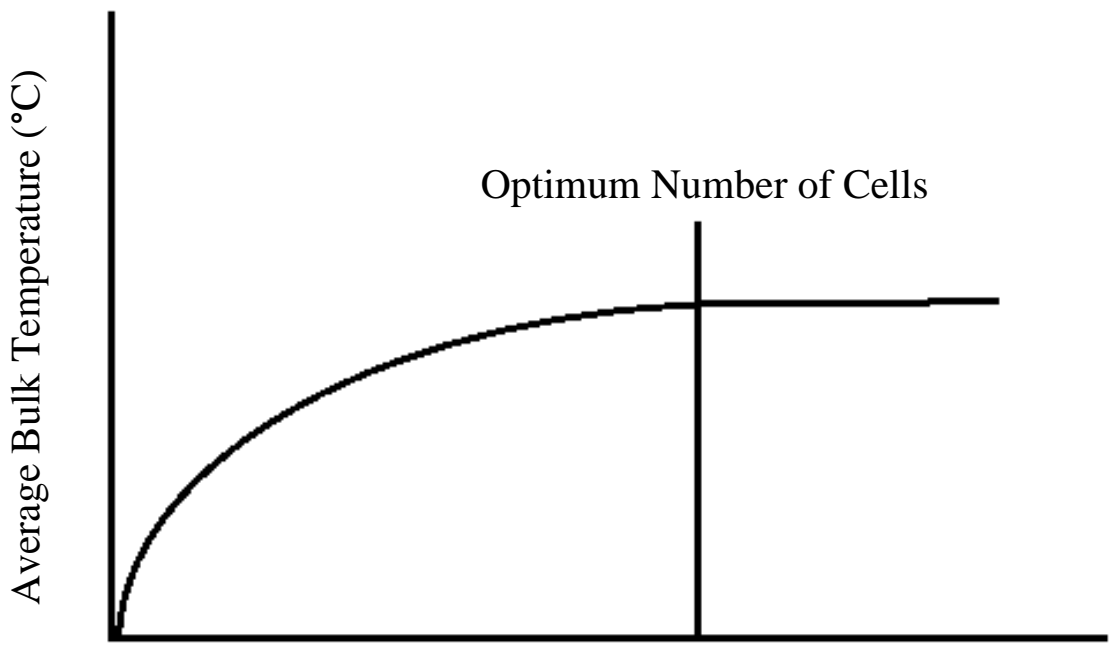

Number of Cells in Mesh

Figure 3.5. Mesh convergence analysis comparing the number of cells in a mesh and a corresponding ABT on a cross sectional plane.

On the other hand, if the mesh contained very small cells so that the number of cells in the mesh approached infinity, the mesh would require extensive computational times and would only vary the resultant temperature slightly. Therefore, a mesh convergence analysis is required to obtain an optimum number of cells so that resulting temperatures are most accurate and computational times are at a minimum. 
Siemens STAR-CCM+. Siemens STAR-CCM+® is a commercially available finite element analysis software platform used to solve complex and accurate physics simulations including fluid dynamics, heat and mass transfer, solid mechanics, and related phenomena [133]. All of the models in this study were executed using STAR$\mathrm{CCM}+$ (version 13.04.010), where FVM was employed as a discretization process that transformed PDE's into explicit algebraic equations so that numerical solutions could be determined. In Chapter 6, the computational domains will be described as a mesh with appropriate discretization for solving fluid flow and temperature distributions in patientspecific coronary arteries.

A synonymous description for STAR-CCM+ modeling is the term computational fluid dynamics (CFD), which refers to the use of high-speed computers for computing mathematical simulations of real-life physical events or processes [114]. The goal of CFD software packages is to analyze and predict fluid flow, heat and mass transfer, and chemical reactions using numerical simulations to solve for conservation of mass, momentum, and energy [114]. CFD analysis offers a comparable alternative to traditional experimentation and testing by providing a low cost, finite element model substitution. Regardless of which software package is used for CFD simulations, the same approach is required to simplify and solve for the solution. The typical CFD workflow is best described by the following flowchart (Fig. 3.6): 
IDENTIFY MODELING GOALS

PRE-PROCESSING

- Define model geometry/domain

- Create mesh

- Set material properties

- Set boundary \& initial conditions

- Select modeling physics

\section{CFD SOLVERS}

- Define Governing Equations

- Identify convergence criteria

- Employ monitor plots

- Initialize solution

\section{POST-PROCESSING}

- Residuals plot

- Velocity profile

- $\mathrm{X}-\mathrm{Y}$ graphs

Figure 3.6. The general CFD modeling approach that describes the necessary steps for preparing and appropriately executing a CFD simulation [114].

All CFD simulations use a similar approach to Fig. 3.6. The first step is to clearly define the aims of the simulation prior to defining the geometry and creating the mesh. Additionally, one must ensure that the correct physics models are employed in the solver, and that the solution fully converges. Since CFD modeling provides an alternative to experimental development, CFD studies must always be scrutinized and compared with experimental results and published data to validate each model's credibility [134]. 
Computer hardware. All Mimics ${ }^{\circledR}$ modeling, SolidWorks ${ }^{\circledR}$ editing, and STAR$\mathrm{CCM}+{ }^{\circledR}$ simulations were executed using an XI M Tower 2P64X Workstation with a 3.70 GHz Intel Xeon Quad-Core processor and 32.0 GB of RAM running Windows 10 Pro 64-bit Operating System. This workstation will be referred to in Chapter 6 as workstation 1 (WS1). Minor post-processing manipulations including custom plane creation along the artery pathway, cross-sectional ABT reports, and scalar temperature and streamline images of the LMCA were executed using a Dell Inspiron 157000 Series with a $1.60 \mathrm{GHz}$ Intel Core i5 processor and 8.0 GB of RAM running Windows 10 Education 64-bit Operating System. This workstation will be referred to in Chapter 6 as workstation 2 (WS2).

Modeling objective. In summary, this chapter describes the workflow from obtaining DICOM image files to creating patient-specific 3D LMCA models for CFD simulations. Since the goal is to create thermal fluid models of $\mathrm{TH}$ in six patient-specific human LMCAs, this chapter serves as a foundation to solving the thesis objectives. 


\section{Chapter 4 \\ In Vitro Methods}

\section{In Vitro Setup}

This chapter presents the in vitro mock loop, including the equipment and calibration methods used to measure temperate and flows in a cardiovascular system. The objective of the in vitro mock loop testing was to use the GuideLiner® outlet temperature and flowrate data as inlet conditions in our computational fluid dynamics (CFD) models.

This in vitro study replicated physiological blood flow and temperature conditions and provided realistic data to use in our CFD simulations. The mock loop system provided a rapid and accesible phyioslogical enviroment to accurately test and record the outlet temperatures and flow rates for the 7F Cordis Vista Brite ${ }^{\circledR}$ guide and guide extension in conditions similar to in vivo.

The following bullets are steps needed to obtain this objective:

- Calibrate thermocouples, pressure sensors, and flow meters.

- Update and execute mock loop protocol.

- Place the Cordis Vista Brite ${ }^{\circledR} 7 \mathrm{~F}$ guide catheter in the mock loop and measure Vascular Solutions GuideLiner@ outlet temperatures for a variety of flowrates using distilled water. In this chapter, the guide catheter will be called the "guide" and the GuideLiner ${ }^{\circledR}$ will be called the "guide extension".

Mock loop geometry. An in vitro mock loop from Merrill et al. [93][94] was rebuilt and modified to simulate the cooling performance of a standard catheter in the left main coronary artery (LMCA) vasculature (Fig. 4.1). The in vitro loop comprises of Tygon tubing networks, valves, and fittings that represent a controlled mock loop of the femoral, aortic, and coronary vascular systems. 


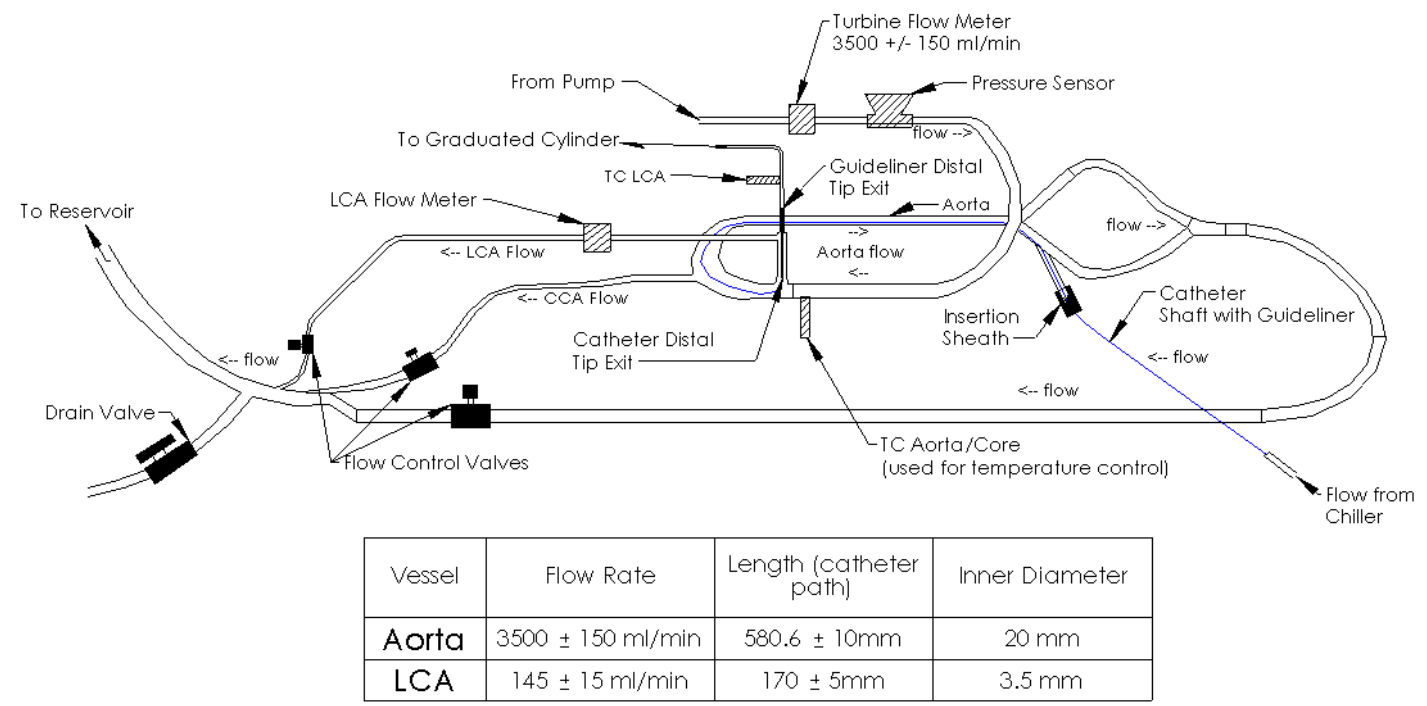

Figure 4.1. Schematic of modified coronary mock loop showing catheter path and mock vessels [93]. The guide (represented by solid blue line) is inserted into the femoral insertion sheath and advanced through the glass aorta into the LMCA ostium. The table at the bottom of the schematic describes the sizes and proper flow rates for vessels of interest.

The cardiovascular mock loop system circulates distilled water at physiological flows, pressures, and temperatures through a glass aorta. A second portable loop system pumps distilled water through an external heat exchanger and into the proximal hub of the guide. The guide was inserted into an insertion sheath through the femoral artery access point of the glass aortic arch and advanced and fixed at the ostium of the LMCA.

A full-size glass replica model of the aortic arch (Vasodyn, Freeville, NY) was positioned at the center of the test loop (Fig 4.2), and from it stems left and right coronary artery access points, as well as a femoral artery access point for blood (distilled water) removal and catheter insertion. 


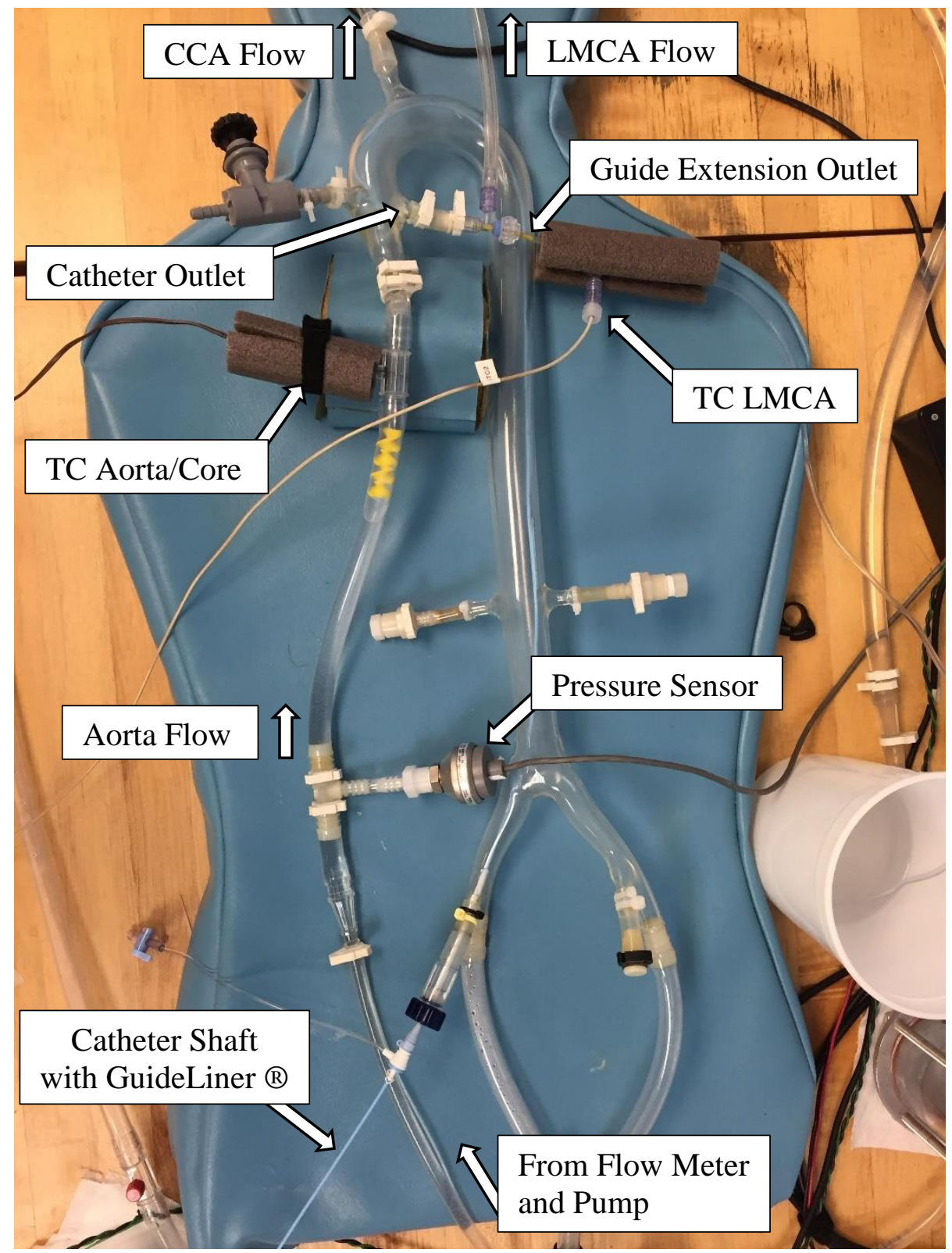

Figure 4.2. Image of coronary mock loop showing catheter path and mock vessels. Note: Thermocouple (TC), common carotid artery (CCA), and left main coronary artery (LMCA). Flow from the large reservoir is pumped through a flow meter before entering the Tygon tubing as proximal Aorta flow. Flow enters the Aortic arch and disperses into the coronary artery ostia where it continues to the left main coronary artery (LMCA) and the guide extension outlet. Aortic flow exits through the common carotid artery (CCA) and femoral arteries downstream.

Rather than a glycerol water mixture, which more closely resembles blood properties, we chose to use distilled water. The justification for using distilled water as a 
fluid in the in vitro mock circulatory loop was due to the forced convection parameters from the non-dimensional Reynolds (Eq. 4.1) and Prandtl numbers (Eq. 4.2) [115][116]:

$$
\begin{aligned}
& R e=\frac{\rho V D}{\mu} \\
& P r=\frac{C_{p} \mu}{\kappa}
\end{aligned}
$$

Where $\rho$ is density $\left(\frac{\mathrm{kg}}{\mathrm{m}^{3}}\right), V$ is velocity $\left(\frac{\mathrm{m}}{\mathrm{s}}\right), D$ is diameter $(m), \mu$ is dynamic viscosity $(P a \cdot s), C_{p}$ is specific heat $\left(\frac{\mathrm{J}}{\mathrm{kg} \cdot \mathrm{K}}\right)$, and $\kappa$ is thermal conductivity $\left(\frac{\mathrm{W}}{\mathrm{m} \cdot \mathrm{K}}\right)$.

The Reynolds number is a dimensionless value that describes the ratio of inertial to viscous forces and characterizes laminar $(\operatorname{Re}<2300)$, transient $(2300<\operatorname{Re}<4000)$ or turbulent flow $(\operatorname{Re}>4000)$ acting in a fluid [115]. The Prandtl number is a dimensionless parameter that characterizes the ratio of diffusion for momentum with respect to heat within a fluid [116]. The dimensionless values for Equation 4.1 and 4.2 are compared to each other and presented in Table 4.1.

Table 4.1. Dimensionless values for Reynolds and Prandtl numbers assuming 68.85

\begin{tabular}{|c|c|c|c|c|}
\hline Fluid & $\operatorname{Re}$ & $\operatorname{Pr}$ & $\operatorname{Re} x \operatorname{Pr}$ & $\begin{array}{c}\operatorname{Re} \times \operatorname{Pr} \\
\text { Difference }(\%)\end{array}$ \\
\hline $\begin{array}{c}\text { Distilled } \\
\text { Water }\end{array}$ & 487 & 7.0 & 3404.2 & \multirow{2}{*}{2.05} \\
\hline Blood & 162 & 20.5 & 3334.2 & \\
\hline
\end{tabular}
$\mathrm{mL} / \mathrm{min}$ inlet flow rate and $0.003 \mathrm{~m}$ inlet artery diameter.

The difference in dynamic viscosity between the blood (0.003 $\mathrm{Pa} \cdot \mathrm{s})$ and distilled water $(0.001 \mathrm{~Pa} \cdot \mathrm{s})$ resulted in a $100.15 \%$ overall difference in Re. However, a small difference of $2.05 \%$ resulted between comparing $(\mathrm{Re}) \mathrm{x}(\mathrm{Pr})$ of the blood and distilled 
water. Since these latter differences are small, the distilled water should closely predict warming. Therefore, the distilled water was chosen for ease of use in this pilot work.

A large reservoir containing distilled water (Fig. 4.3) is circulated and maintained at physiological temperatures $\left(37^{\circ} \mathrm{C} \pm 5\right)$ using an immersion heater (George Ulanet Co. Newark, NJ) controlled by a temperature feedback controller (Cole-Parmer, Vernon Hills, IL) and peristaltic circulation pump (Ismatec SA, Glattbrugg, Switzerland). A custom dampening cylinder is secured downstream to the peristaltic pump to reduce vibration at high flow rates.

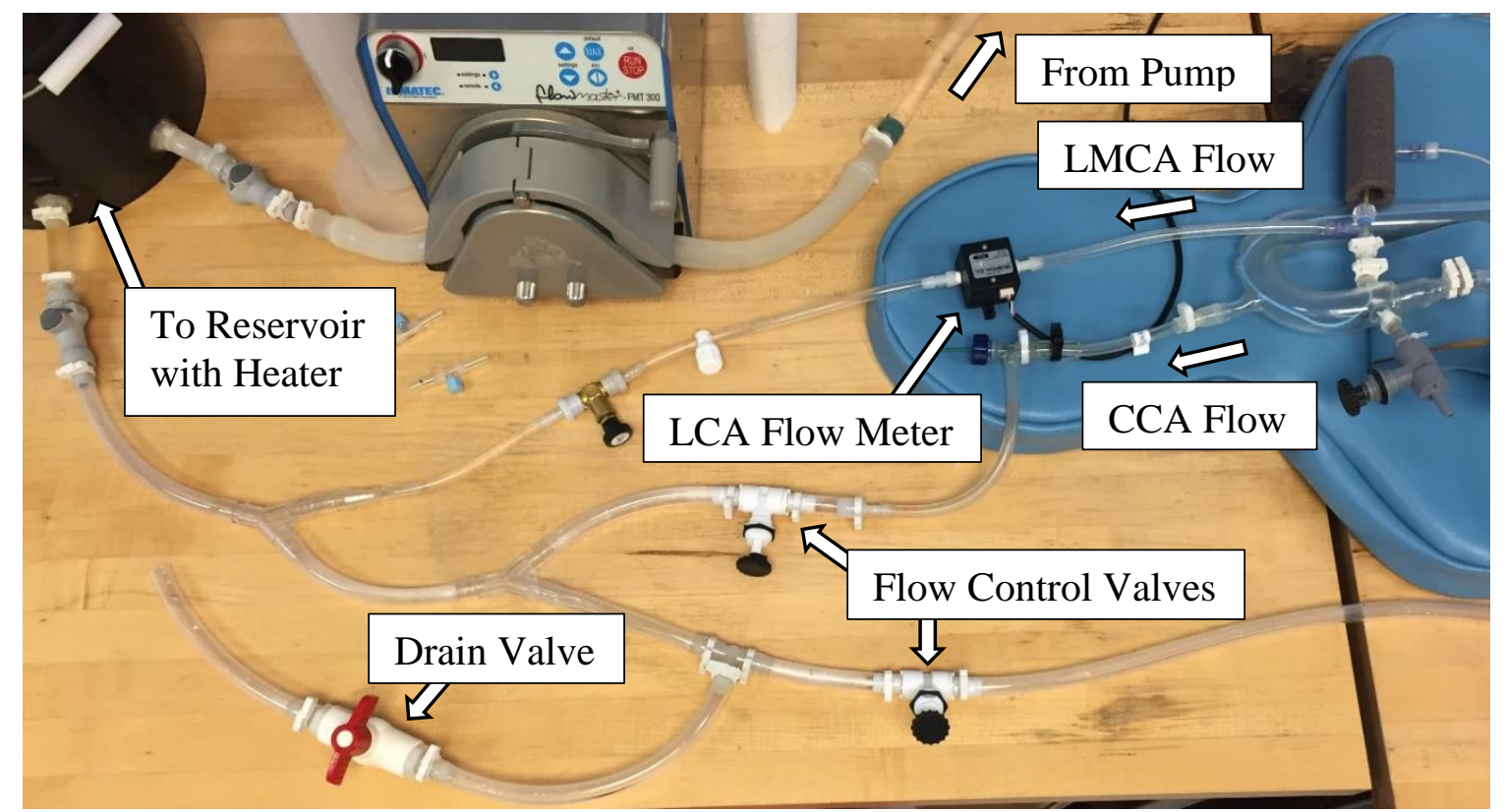

Figure 4.3. Continuation of Fig. 4.2 showing catheter path and mock vessels. Distilled water is heated in the reservoir and pumped into circulation via the peristaltic pump. Flow to the common carotid artery (CCA) and left main coronary artery (LMCA) can be adjusted using flow control valves downstream.

Flow rates. Distilled water was used as the chilled infusate flow through the catheter at $10 \mathrm{~mL} / \mathrm{min}$ to $90 \mathrm{~mL} / \mathrm{min}$ flowrates, as well as the blood throughout the system. Flow was varied using control valves to alter flow dispersions throughout the 
mock circulatory loop. Flow was measured at various locations throughout the loop using two turbine flow meters (McMillan, Georgetown, TX), and a transonic flow meter (Transonic Systems Inc., Ithaca, NY). Equipment specifications and employed use for each flow meter are listed later in this chapter.

To circulate and measure blood flow about the catheter in the coronary lumen, a Tuohy Borst Adapter (Fig. 4.4) with side port (Qosina, Ronkonkoma, NY) was connected to the LMCA ostium.

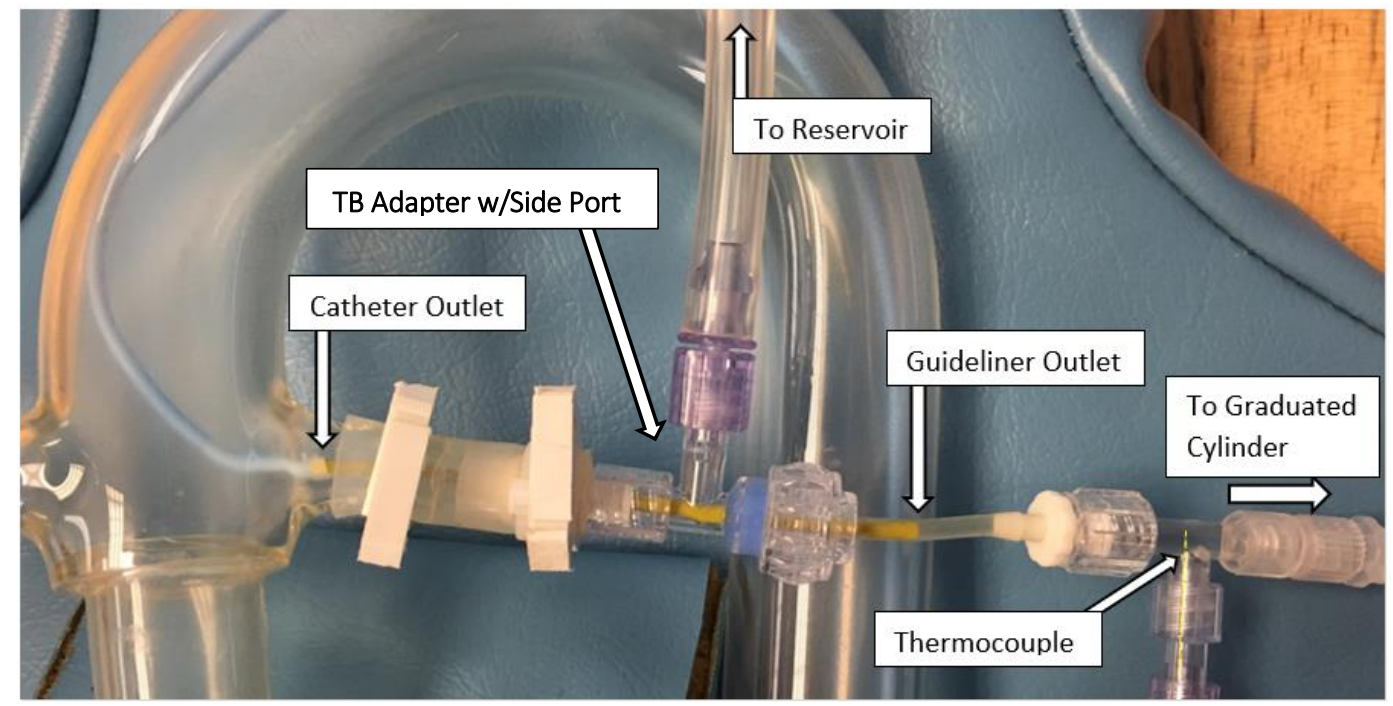

Figure 4.4. The Cordis ${ }^{\circledR}$ catheter enters the ostium of the LMCA and the guide extension is advanced $8.5 \mathrm{~cm}$ through the Tuohy borst adapter (TB Adapter), out of the catheter, and positioned in tight fit tubing. The fittings and catheter are insulated (not shown) after assembly. The "To Reservoir" label represents flow advancing towards the LMCA flow meter and into the mock loop reservoir. The "To Graduated Cylinder" label refers to infusate flowing towards the reservoir (or graduated cylinder), which is used to measure guide extension flowrate.

The sideport redirected coronary flow to a turbine flow meter (McMillan, Georgetown, TX) and into the large reservoir. The guide extension was advanced $8.5 \mathrm{~cm}$ out of the catheter and through the Tuohy Borst outlet. Tight fit tubing extended the guide extension and a type-T thermocouple was positioned $3 \mathrm{~cm}$ distal to the Touhy Borst 
outlet and used to measure the temperature of the chilled infusate leaving the catheter. To ensure that average bulk temperatures were being measured, the entire flow path would be insulated from the exit of the GuideLiner® to the thermocouple, and would incorporate an in-line static mixer directly upstream of the thermocouple. For this work, the distance between the distal tip of the GuideLiner ${ }^{\circledR}$ and the temperature measurement point was minimized, which reduced heat loss and the need for insulation, and the fittings between the distal tip and temperature sensing location mixed the fluid at the point of the thermocouple.

Flow rates at critical locations in the in vitro mock loop are described in Table

\section{2 .}

Table 4.2

Critical flowrates and locations in mock circulatory loop.

\begin{tabular}{ccl} 
Location in Mock Loop & Flow rate $(\mathrm{ml} / \mathrm{min})$ & \multicolumn{1}{c}{ Reference } \\
\hline Aorta & $3500 \pm 150$ & {$[93]$} \\
\hline LMCA & $145 \pm 15$ & {$[10][11][12]$} \\
\hline Guide Extension Outlet & $10-90 \mathrm{ml} / \mathrm{min}$ & {$[13][96]$} \\
\hline
\end{tabular}

Temperatures. Type-T thermocouples (Physitemp, Clifton, NJ and Blaze

Technical Services, Stow, $\mathrm{OH}$ ) were used to measure the catheter inlet and outlet, aorta, and heat exchanger outlet temperatures Table 4.3. 
Table 4.3

Make and sizes of thermocouples used to measure guide inlet, guide extension outlet, heat exchanger, and aorta temperatures.

\begin{tabular}{|c|c|c|c|c|c|}
\hline TC Location & Make & Model Number & $\begin{array}{l}\text { Sheath } \\
\text { Diameter }\end{array}$ & $\begin{array}{l}\text { Needle } \\
\text { (Tip) } \\
\text { Length }\end{array}$ & TC Length \\
\hline Guide Inlet & Physitemp & MT26-2 HT & $0.018^{\prime \prime}$ & $2 \mathrm{~cm}$ & $5 \mathrm{ft}$ \\
\hline $\begin{array}{c}\text { Guide } \\
\text { Extension } \\
\text { Outlet }\end{array}$ & Physitemp & MT26-2 HT & $0.018^{\prime \prime}$ & $2 \mathrm{~cm}$ & $5 \mathrm{ft}$ \\
\hline $\begin{array}{c}\text { Heat } \\
\text { Exchanger }\end{array}$ & Physitemp & MT26-2 HT & $0.018^{\prime \prime}$ & $2 \mathrm{~cm}$ & $5 \mathrm{ft}$ \\
\hline Aorta (Core) & Omega & TMQSS 040G & $0.040^{\prime \prime}$ & 6 in & N/A \\
\hline
\end{tabular}

Note . Thermocouple $=$ TC .

Type-T Thermocouple accuracy as rated by the manufacturer is $\pm 0.1{ }^{\circ} \mathrm{C}$. If noise or differences in simulation results for $\mathrm{ABT}$ are without the range of $\pm 0.1^{\circ} \mathrm{C}$ then the results will not be accurately measured and values within this range will be disregarded.

A high-accuracy pressure transducer (Cole-Parmer, Vernon Hills, IL) measured the pressure entering the aorta, and all instrumented measurement data was recorded using LABVIEW (National Instruments, Austin, TX) DAQ software.

A total of 5 thermocouples were used in the mock loop system to measure the temperature at the catheter inlet and outlet, Aorta (core body temperature), chilled distilled water leaving the heat exchanger, and the Ambient (room) temperature. The mock loop core temperature, $37^{\circ} \mathrm{C}$, was regulated by the reservoir heater and LabView control algorithm and was measured using a type-T thermocouple positioned upstream to the LMCA ostium in the glass aorta. The external cooling console (Fig. 4.5) was set to $0^{\circ} \mathrm{C}$ and pumped the chilled distilled water through a heat exchanger and into the proximal end of the guide. 


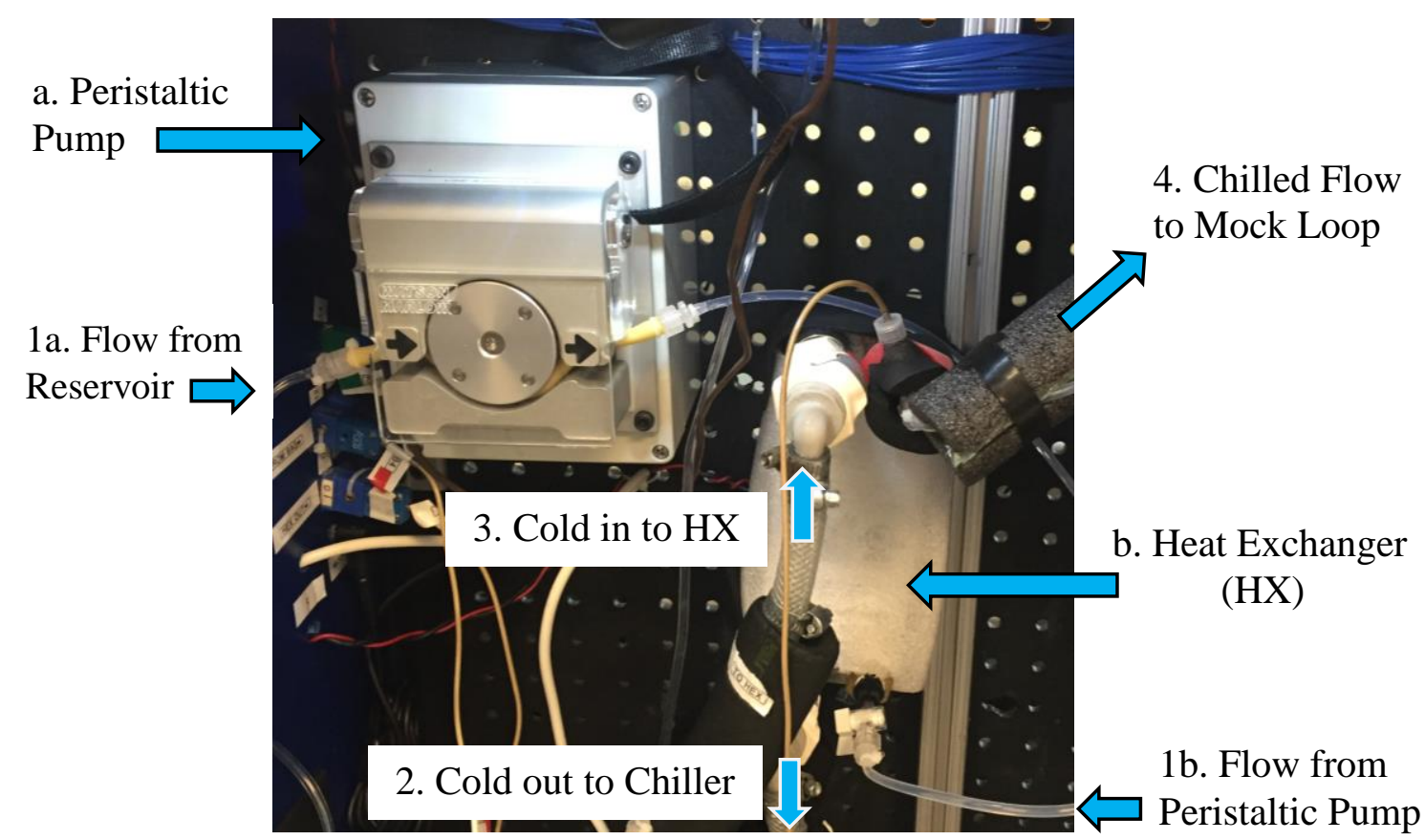

Figure 4.5. Internal view of custom built portable cooling console. The peristaltic pump (a) circulates flow from a small reservoir to the heat exchanger (b). Distilled water is chilled through the heat exchanger and advanced into the mock circulatory loop (4) by way of the Guide Catheter.

Chilled flow entering the proximal hub of the Cordis catheter was pumped using a custom built portable cooling console. The portable cooling console was mounted on a cart for easy maneuverability and Tygon tubing was used to join the cooling console to the catheter inlet and the rest of the components. This cooling console was used to circulate chilled saline (distilled water) at $10 \mathrm{ml} / \mathrm{min}$ to $90 \mathrm{ml} / \mathrm{min}$ through the catheter by first chilling the distilled water to $0^{0} \mathrm{C}$. The chilled infusate is pumped from a small reservoir to the catheter via a peristaltic pump (Watson-Marlow Fluid Technology Group, Wilmington, MA). A thermoelectric chiller (Custom Chill Inc., Philadelphia, PA) and a flat plate heat exchanger (GEA PHE Systems NA, York, PA) is used to chill the infusate to the desired temperature, $0^{\circ} \mathrm{C}$. 


\section{Equipment}

Various equipment was used for the mock loop system, including flow meters, pressure sensors, thermocouples, pumps, fittings, and computer hardware. This section describes the make and models, calibration ranges, and medical devices used in the mock circulatory loop system.

Make and models. Table 4.4 lists the makes and models of the equipment used to replicate and measure physiological conditions in the in vitro circulatory mock loop system.

Table 4.4

Make and model of flow meters, thermocouples, and pressure transducer used in mock circulatory loop system.

\begin{tabular}{|lll|}
\hline $\begin{array}{l}\text { Description } \\
\text { Coronary Flow }\end{array}$ & $\begin{array}{l}\text { Instrument } \\
\text { Turbine Flow Meter }\end{array}$ & $\begin{array}{l}\text { Make \& Model } \\
\text { McMillan Co., Georgetown, } \\
\text { TX, S/N 11744 }\end{array}$ \\
\hline Core Flow & Turbine Flow Meter & $\begin{array}{l}\text { McMillan Co., Georgetown, } \\
\text { TX, S/N 1616 }\end{array}$ \\
\hline $\begin{array}{l}\text { Cath. Inlet \& Outlet, } \\
\text { HX, ambient temp }\end{array}$ & Type-T Thermocouples & Physitemp, Clifton, NJ \\
\hline $\begin{array}{l}\text { Aorta (core) temp } \\
\text { Aorta Pressure }\end{array}$ & Thermocouple & Omega, Norwalk, CT \\
\hline
\end{tabular}

Note. Heat Exchanger $=\mathrm{HX}$.

A description of the peristaltic pump, Tuohy Borst adapters, and full-size glass aortic arch employed in the mock loop system are listed in Table 4.5. 
Table 4.5

Make and model of peristaltic pumps, glass aorta, and Tuohy Borst adapters employed in the mock circulatory loop.

\begin{tabular}{|c|c|c|}
\hline Description & Instrument & Make \& Model \\
\hline Circulatory Pump & Peristaltic Pump & Ismatec, Glattbrugg, Switzerland \\
\hline Infusate Pump & Peristaltic Pump & Watson-Marlow, Wilmington, MA \\
\hline $\begin{array}{l}\text { In-Circuit } \\
\text { Dampener }\end{array}$ & Custom Dampener & FocalCool, LLC, Mullica Hill, NJ \\
\hline Aortic Arch & $\begin{array}{l}\text { Full-Size Glass } \\
\text { Aorta }\end{array}$ & Vasodyn, Freeville, NY \\
\hline LMCA & $\begin{array}{l}\text { Tuohy Borst } \\
\text { Adapter w/Sideport }\end{array}$ & $\begin{array}{l}\text { Qosina, Ronkonkoma, NY, P/N } \\
80460\end{array}$ \\
\hline
\end{tabular}

The immersion heater used to heat the large reservoir (George Ulanet Co.

Newark, NJ) was controlled by a temperature feedback controller (Cole-Parmer, Vernon

Hills, IL) connected to a Thinkpad Ultrabook Intel core I5 vpro that was automated using

LabVIEW (National Instruments Co., Austin, TX) data acquisition (DAQ) software.

Range. Each thermocouple was calibrated to a confidence range that

corresponded to a specific physiological temperature local to its intended use. For

instance, the thermocouple for the aorta was calibrated for a confidence range between

$35^{\circ} \mathrm{C}$ and $38^{\circ} \mathrm{C}$, which was used to measure core body temperature, $37^{\circ} \mathrm{C}$. The confidence ranges for each of the thermocouples are found in Table 4.6.

Table 4.6

Operational ranges for each thermocouple used in the mock loop system.

\begin{tabular}{|l|cc|}
\hline Thermocouples: & Range $\left({ }^{\circ} \mathrm{C}\right)$ & Confidence Range $\left({ }^{\circ} \mathrm{C}\right)$ \\
\hline Cath Inlet Temp. & $-1.0-15$ & $-1.0-18$ \\
\hline HX Outlet Temp. & $-1.0-15$ & $-1.0-18$ \\
\hline Ambient Temp. & $15-25$ & $17-28$ \\
\hline Aorta (core) Temp. & $35-38$ & $32-38$ \\
\hline Cath Outlet Temp. & $6.0-30$ & $5.0-31$ \\
\hline
\end{tabular}


Akin to the predetermined thermocouple ranges, the flow meters were also calibrated to physiological flows and are listed in Table 4.7.

Table 4.7

Operational ranges for each instrument used to measure flow rate and pressure.

\begin{tabular}{|lcc|}
\hline Flows: & Range & Confidence Range \\
\hline Aorta & $3000-4000 \mathrm{ml} / \mathrm{min}$ & $1000-4000 \mathrm{ml} / \mathrm{min}$ \\
\hline Coronary & $70-200 \mathrm{ml} / \mathrm{min}$ & $82-183 \mathrm{ml} / \mathrm{min}$ \\
\hline $\begin{array}{l}\text { Pressures: } \\
\begin{array}{l}\text { Catheter } \\
\text { Inlet }\end{array}\end{array}$ & Range & Confidence Range \\
\hline Aorta & $0-30 \mathrm{psi}$ & $7-18 \mathrm{PSI}$ \\
\hline
\end{tabular}

Calibration and precision. Calibration methods were performed for the thermocouples, flow meters, and pressure sensors. The thermocouples were calibrated in a chilled liquid bath (Thermo NESLAB, Oak Park, IL) consisting of 50\% glycerol and $50 \%$ distilled water so that $0^{\circ} \mathrm{C}$ temperatures could be reached. Flow meters were calibrated using gavimetric methods and pressure sensors were calibrated using known water column heights. All sensor calibrations were within the manufacturer specifications.

The tips of the thermocouples were positioned $\sim 1.5$ inches into the chiller bath solution and the liquid bath ranged between $-3^{\circ} \mathrm{C}$ and $40^{\circ} \mathrm{C}$ as LabVIEW was used to record 60 temperature data points (1 point per second) for each thermocouple.

Each thermocouple was calibrated within a specific confidence range around its working temperature in the mock loop system. A mock loop test calibration guide (Table 4.8) for the operational ranges for each thermocouple was created to keep track of each thermocouple's calibration parameters. 
Table 4.8

Thermocouple calibration ranges, calibration points \& accuracy, and calibration check method.

\begin{tabular}{|c|c|c|c|c|}
\hline Thermocouples & $\begin{array}{l}\text { Range } \\
\left({ }^{\circ} \mathrm{C}\right)\end{array}$ & $\begin{array}{l}\text { Calibration Points } \\
\qquad\left({ }^{\circ} \mathrm{C}\right)\end{array}$ & $\begin{array}{c}\text { Calibrated } \\
\text { Accuracy } \\
\left({ }^{\circ} \mathrm{C}\right)\end{array}$ & $\begin{array}{l}\text { Calibration } \\
\text { Check }\end{array}$ \\
\hline Cath Inlet Temp & $-1.0-15$ & $-3,0,3,7,12,15,20$ & \pm 0.28 & \multirow{5}{*}{$\begin{array}{l}\text { Generated flow } \\
\text { vs voltage plot. } \\
\text { Obtained linear } \\
\text { regression slope } \\
\text { and y-intercept. }\end{array}$} \\
\hline HX Outlet Temp & $-1.0-15$ & $-3,0,3,7,12,15,20$ & \pm 0.55 & \\
\hline Ambient Temp & $15-25$ & $12,15,20,23,25,30$ & \pm 0.38 & \\
\hline $\begin{array}{l}\text { Aorta (core) } \\
\text { Temp }\end{array}$ & $35-40$ & $30,33,35,37,40$ & \pm 0.25 & \\
\hline Cath Outlet & $6.0-30$ & $3,7,15,23,30,33$ & \pm 0.36 & \\
\hline
\end{tabular}

A linear regression and $\mathrm{R}^{2}$ value was determined for each thermocouple data set to validate the accuracy of the calibration. The new slope and intercept value from each thermocouple's linear regression plot was added to the LabVIEW DAQ as a new calibration input value.

The process for calibrating flow meters and pressure transducers was similar to the thermocouple calibration method. A total of five calibration points were spread evenly throughout the range of flows and pressures (Table 4.9) we intended to measure. 
Table 4.9

Coronary test calibration guide for the operational ranges for each instrumentContinued.

\begin{tabular}{|c|c|c|c|c|}
\hline Flows: & Range & Calibration Points & $\begin{array}{l}\text { Calibrated } \\
\text { Accuracy }\end{array}$ & Calibration Check \\
\hline Aorta & $\begin{array}{c}3000-4000 \\
\mathrm{ml} / \mathrm{min}\end{array}$ & $\begin{array}{c}2000,2500,3000 \\
3500,4000\end{array}$ & $\begin{array}{c} \pm 200 \\
\mathrm{ml} / \mathrm{min}\end{array}$ & $\begin{array}{c}\text { Checked calibration } \\
\text { - no recalibration } \\
\text { necessary }\end{array}$ \\
\hline Coronary & $\begin{array}{l}70-200 \\
\mathrm{ml} / \mathrm{min}\end{array}$ & $\begin{array}{c}60,100,130,170 \\
200\end{array}$ & $\begin{array}{l} \pm 1.04 \\
\mathrm{ml} / \mathrm{min}\end{array}$ & $\begin{array}{c}\text { Checked with water } \\
\text { weight of graduated } \\
\text { cylinder divided by } \\
\text { time }\end{array}$ \\
\hline Pressure: & Range & Calibration Points & $\begin{array}{l}\text { Calibrated } \\
\text { Accuracy }\end{array}$ & Calibration Check \\
\hline $\begin{array}{l}\text { Catheter } \\
\text { Inlet }\end{array}$ & $0-30 \mathrm{psi}$ & $0,5,10,20,30,35$ & \pm 0.48 psig & $\begin{array}{c}\text { Checked with } \\
\text { hydraulic apparatus }\end{array}$ \\
\hline Aorta & $\begin{array}{c}50-170 \\
\mathrm{mmHg}\end{array}$ & $40,80,120,140,170$ & $\begin{array}{l} \pm 1.46 \\
\mathrm{mmHg}\end{array}$ & $\begin{array}{l}\text { Checked with water } \\
\text { column }\end{array}$ \\
\hline
\end{tabular}

After the data was recorded using LabVIEW, it was plotted in Excel and a new slope was determined. A linear trend line was added to each graph, as well as the $\mathrm{R}^{2}$ value. An $\mathrm{R}^{2}$ value greater than 0.98 was considered satisfactory for the turbine flow meter and pressure sensor calibrations. Coronary and aorta flow were calibrated with an accuracy of $\pm 1.04 \mathrm{ml} / \mathrm{min}$ and $\pm 27 \mathrm{ml} / \mathrm{min}$, respectively. The Aorta flow meter was within the $\pm 200 \mathrm{ml} / \mathrm{min}$ acceptable limit for the calibration, therefore, no recalibration was needed. The inputs, control variables, and outputs employed in the calibration experiments are summarized as a flowchart in Figure 4.6. 


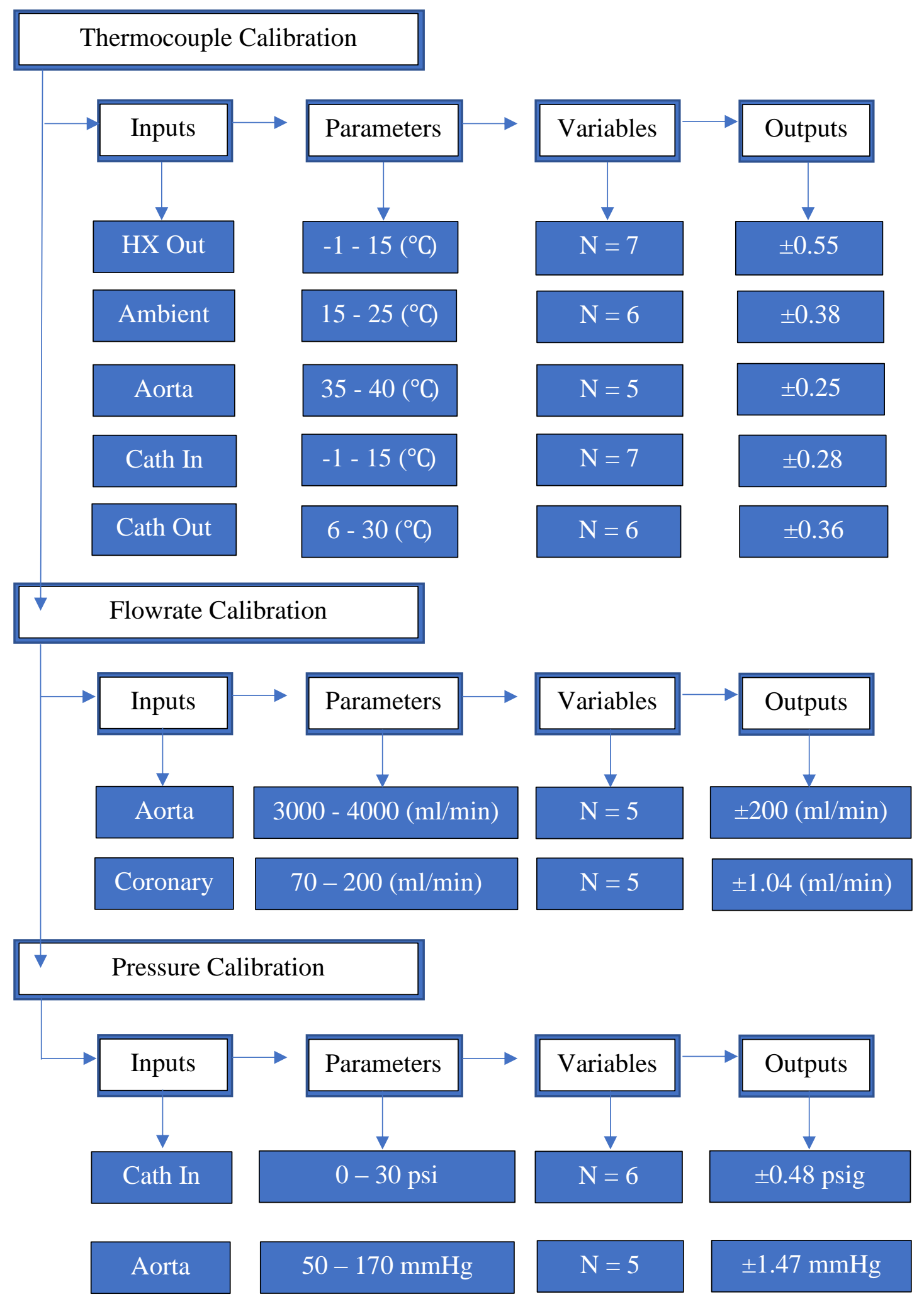

Figure 4.6. Snapshot of the calibration design of experiments for thermocouple, flow, and pressure calibration with each corresponding input, output, parameter, and variable. 
To calibrate infusate flow rates, the peristaltic pump in the cooling console varied flow between 10 and $90 \mathrm{ml} / \mathrm{min}$ which was collected and measured in a graduated cylinder. The calibrated measurements were updated in the DAQ system and are listed in Table 4.10.

Table 4.10

Calibrated infusate flowrates exiting the guide extension from peristaltic pump in the cooling console.

\begin{tabular}{|cccccccc|}
\hline $\begin{array}{c}\text { Description } \\
\text { Pump Flow Rate }\end{array}$ & $\mathbf{1 0}$ & $\mathbf{2 0}$ & $\mathbf{3 0}$ & $\mathbf{5 0}$ & $\mathbf{7 0}$ & $\mathbf{9 0}$ & \\
\hline Calibrated Flowrate & 9.08 & 19.3 & 29.20 & 49.0 & 68.8 & 88.9 & $\mathbf{~ m l} / \mathbf{m i n}$ \\
\hline Total Uncertainty & 0.03 & 0.1 & 0.08 & 0.2 & 0.2 & 0.4 & $\mathbf{m l} / \mathbf{m i n}$ \\
\hline
\end{tabular}

The catheter inlet pressure and distilled water flow rates were measured using a pressure transducer (PendoTech, Princeton, NJ) and a turbine flow meter (McMillan, Georgetown, TX), respectively. The temperature and flow rate of the catheter was monitored and controlled by the user with a computer running LABVIEW (National Instruments Co., Austin, TX).

Medical devices. The medical devices used in this study (Table 4.11) comprised of a 7F Cordis ${ }^{\circledR}$ guide and a 7F guide extension that was advanced through the guide to the artery of interest.

Table 4.11

Make and model for each medical device used in the mock loop system. Device Model Description

7F Guiding Catheter

Cordis Vista BriteTip® (Part Number: JL 3.5)
Vascular Solutions (Part Number: 5572) 


\section{Results of In Vitro Studies}

In this section we present the temperatures and flow rates at the guide inlet and guide extension outlet, as well as the cooling capacity of the Cordis ${ }^{\circledR}$ guide for all temperatures and flow rates used in the mock loop system. The term, infusate flow, will be referred to in this section as the flow being pumped from the cooling console and exiting the tip of the guide extension.

Temperatures. Aorta (core) temperatures were held constant at $37.07^{0} \mathrm{C} \pm 0.046$ for all the mock loop trials. The Calibrated Infusate flow rates ranged between 9.08 and $88.9 \mathrm{ml} / \mathrm{min}$ (Table 4.12), and the temperatures for the heat exchanger outlet, catheter inlet, and guide extension outlet were recorded.

Table 4.12

Various temperatures recorded from infusate flow rates ranging from $9.08 \mathrm{ml} / \mathrm{min}$ to 88.9 $\mathrm{ml} / \mathrm{min}$ in the cooling console circuit.

\begin{tabular}{|c|c|c|c|c|c|c|c|}
\hline Description & Units & \multicolumn{6}{|c|}{ Value } \\
\hline $\begin{array}{l}\text { Calibrated Infusate } \\
\text { Flow Rates }\end{array}$ & $\mathrm{ml} / \mathrm{min}$ & 9.08 & 19.27 & 29.2 & 49.03 & 68.85 & 88.9 \\
\hline $\begin{array}{l}\text { Heat Exchanger } \\
\text { Outlet Temp }\end{array}$ & ${ }^{0} \mathrm{C}$ & 1.83 & 1.34 & 1.1 & 0.92 & 0.91 & 0.83 \\
\hline $\begin{array}{l}\text { Catheter Inlet } \\
\text { Temperature }\end{array}$ & ${ }^{0} \mathrm{C}$ & 8.205 & 4.78 & 3.52 & 2.46 & 2.087 & 1.76 \\
\hline $\begin{array}{l}\text { Guide Extension } \\
\text { Outlet Temperature }\end{array}$ & ${ }^{0} \mathrm{C}$ & 36.31 & 33.36 & 29.4 & 23.16 & 19.2 & 16.36 \\
\hline
\end{tabular}

As seen in Table 4.8, infusate flows in the cooling console circuit retained cooler temperatures with higher flowrates. To visualize this scene, temperatures entering the Cordis ${ }^{\circledR}$ guide and exiting the guide extension tip were plotted (Fig. 4.7) against their corresponding infusate flow rates. 


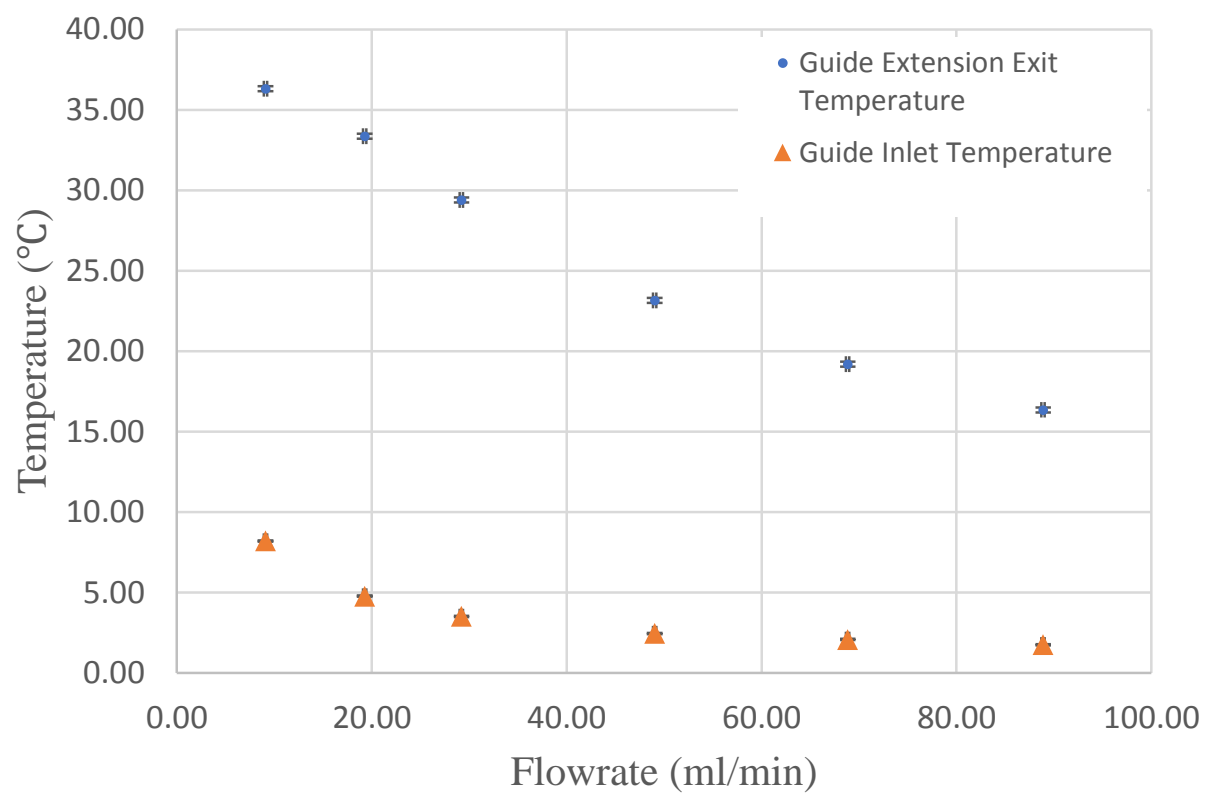

Figure 4.7. Temperatures entering the Cordis ${ }^{\circledR}$ guide and exiting the guide extension vs infusate flow rates from mock loop cooling console. Increased infusate flowrates resulted in lower fluid temperatures both entering and exiting the Cordis ${ }^{\circledR}$ guide and guide extension, respectively. Infusate flowrates leaving the heat exchanger varied between $0.023^{\circ} \mathrm{C}$ and $0.112^{\circ} \mathrm{C}$. Standard. Note: standard deviation error bars are capped.

Flows. The cooling console circulated distilled water from a small reservoir into the heat exchanger and was expelled as chilled fluid $<1^{0} \mathrm{C}$ before entering the guide. Flow rates entering the Cordis ${ }^{\circledR}$ guide ranged between 9.08 and $88.9 \mathrm{ml} / \mathrm{min}$ (Table 4.13) and resulted in varying catheter inlet and outlet temperatures.

Table 4.13

Standard deviation $(S D)$ for flow rates, guide extension inlet temperature, and guide extension outlet temperature.

\begin{tabular}{|llllll|}
\hline Infusate Flow $(\mathrm{ml} / \mathrm{min})$ & \multicolumn{2}{c|}{ Guide Inlet $\left({ }^{\circ} \mathrm{C}\right)$} & \multicolumn{2}{c|}{ Guide Extension Outlet $\left({ }^{\circ} \mathrm{C}\right)$} \\
\hline Flowrate & SD & Temperature & SD & Temperature & SD \\
\hline 9.08 & 0.0289 & 8.205 & 0.0414 & 36.31 & 0.0521 \\
\hline 19.27 & 0.1041 & 4.478 & 0.0555 & 33.36 & 0.3203 \\
\hline 29.2 & 0.0866 & 3.52 & 0.0429 & 29.4 & 0.1881 \\
\hline 49.03 & 0.2309 & 2.459 & 0.0321 & 23.16 & 0.1564 \\
\hline 68.85 & 0.2179 & 2.087 & 0.0141 & 19.2 & 0.0944 \\
\hline 88.9 & 0.3605 & 1.76 & 0.0376 & 16.36 & 0.1253 \\
\hline
\end{tabular}


Catheter inlet temperatures ranged between $1.76^{\circ} \mathrm{C}$ and $8.205^{\circ} \mathrm{C}$ with a standard deviation ranging between $0.0141^{\circ} \mathrm{C}$ and $0.0555^{\circ} \mathrm{C}$ for all six inlet temperatures. The temperatures exiting the guide extension ranged between $16.36^{\circ} \mathrm{C}$ and $36.31^{\circ} \mathrm{C}$ with a standard deviation between $0.0944^{\circ} \mathrm{C}$ and $0.3203^{\circ} \mathrm{C}$ for all six guide extension outlet temperatures.

Cooling capacity. Cooling capacity, $\dot{Q}_{I}$, was calculated using data obtained from the chilled infusate catheter flow rates and guide extension outlet temperatures using equation 4.1:

$$
\dot{Q}_{I}=\dot{m}_{I} c_{p} \Delta T_{I}
$$

where $\dot{Q}_{I}$ is the heat capacity of the infusate in watts, $\dot{m}_{I}$ is the mass flow rate of the chilled infusate in $\mathrm{kg} \mathrm{s}^{-1}, c_{p}$ represents the specific heat of the chilled infusate in $\mathrm{J}$ $\mathrm{kg}^{-1} \mathrm{C}^{-1}$, and $\Delta T_{I}$ represents the delta between the mock loop core temperature and the guide extension outlet temperature in ${ }^{\circ} \mathrm{C}$. The characteristic values for $\dot{m}_{I}, c_{p}$ and $\Delta T_{I}$ were $1.513 \mathrm{e}-4-1.482 \mathrm{e}-3 \mathrm{~kg} \mathrm{~s}^{-1}, 4186 \mathrm{~J} \mathrm{~kg}^{-1} \mathrm{C}^{-1}$, and $0.72-20.7^{\circ} \mathrm{C}$, respectively. The resultant cooling capacity is listed for each infusate flow rate in Table 4.14.

Table 4.14

Data obtained for the infusate flow rates, guide extension outlet temperatures, and aorta temperatures using Eq. 4.1 to solve for cooling capacity.

\begin{tabular}{|c|c|c|c|c|c|}
\hline & $\begin{array}{l}\text { Infusate Flow } \\
(\mathrm{m} 1 / \mathrm{min})\end{array}$ & $\begin{array}{c}\text { Guide } \\
\text { Extension } \\
\text { Outlet }\left({ }^{\circ} \mathrm{C}\right)\end{array}$ & Aorta $\left({ }^{0} \mathrm{C}\right)$ & Delta $\left({ }^{0} \mathrm{C}\right)$ & $\begin{array}{c}\text { Cooling } \\
\text { Capacity (W) }\end{array}$ \\
\hline 1 & $9.08 \pm 0.029$ & $36.31 \pm 0.052$ & $37.028 \pm 0.049$ & $0.72 \pm 0.10$ & $0.45 \pm 0.06$ \\
\hline 2 & $19.27 \pm 0.104$ & $33.36 \pm 0.320$ & $37.023 \pm 0.078$ & $3.67 \pm 0.24$ & $4.93 \pm 0.31$ \\
\hline 3 & $29.2 \pm 0.087$ & $29.40 \pm 0.188$ & $37.116 \pm 0.070$ & $7.72 \pm 0.18$ & $15.72 \pm 0.41$ \\
\hline 4 & $49.03 \pm 0.231$ & $23.16 \pm 0.156$ & $37.068 \pm 0.053$ & $13.91 \pm 0.12$ & $47.58 \pm 0.57$ \\
\hline 5 & $68.85 \pm 0.218$ & $19.20 \pm 0.094$ & $37.033 \pm 0.080$ & $17.83 \pm 0.01$ & $85.65 \pm 0.34$ \\
\hline 6 & $88.9 \pm 0.360$ & $16.36 \pm 0.125$ & $37.061 \pm 0.068$ & $20.7 \pm 0.06$ & $128.41 \pm 0.41$ \\
\hline
\end{tabular}

Note. The \pm values following each entry represent the standard deviation for three data sets. 
It is worth noting from Table 4.10 that infusate flow in line 1 is approximately one-tenth the value of the infusate flow in line 6, whereas corresponding cooling capacities (Eq. 4.1) do not reflect this same ratio. Specific heat, $c_{p}$, in Eq. 4.1 is constant, thus cooling capacity must be contingent upon either mass flowrate, $\dot{m}_{I}$, or the change in fluid temperature, $\Delta T_{I}$. Since fluid temperature in line 6 is two orders of magnitude greater than line 1 , then the resultant cooling capacity ratios become significantly dependent on the $\Delta T_{I}$ along the catheter rather than infusate flow, which varies only by one order of magnitude.

By applying the data obtained for infusate flow and guide extension outlet temperatures in Table 4.11, a plot can be generated (Fig. 4.8) to show cooling capacity as a function of infusate flowrate and temperature.

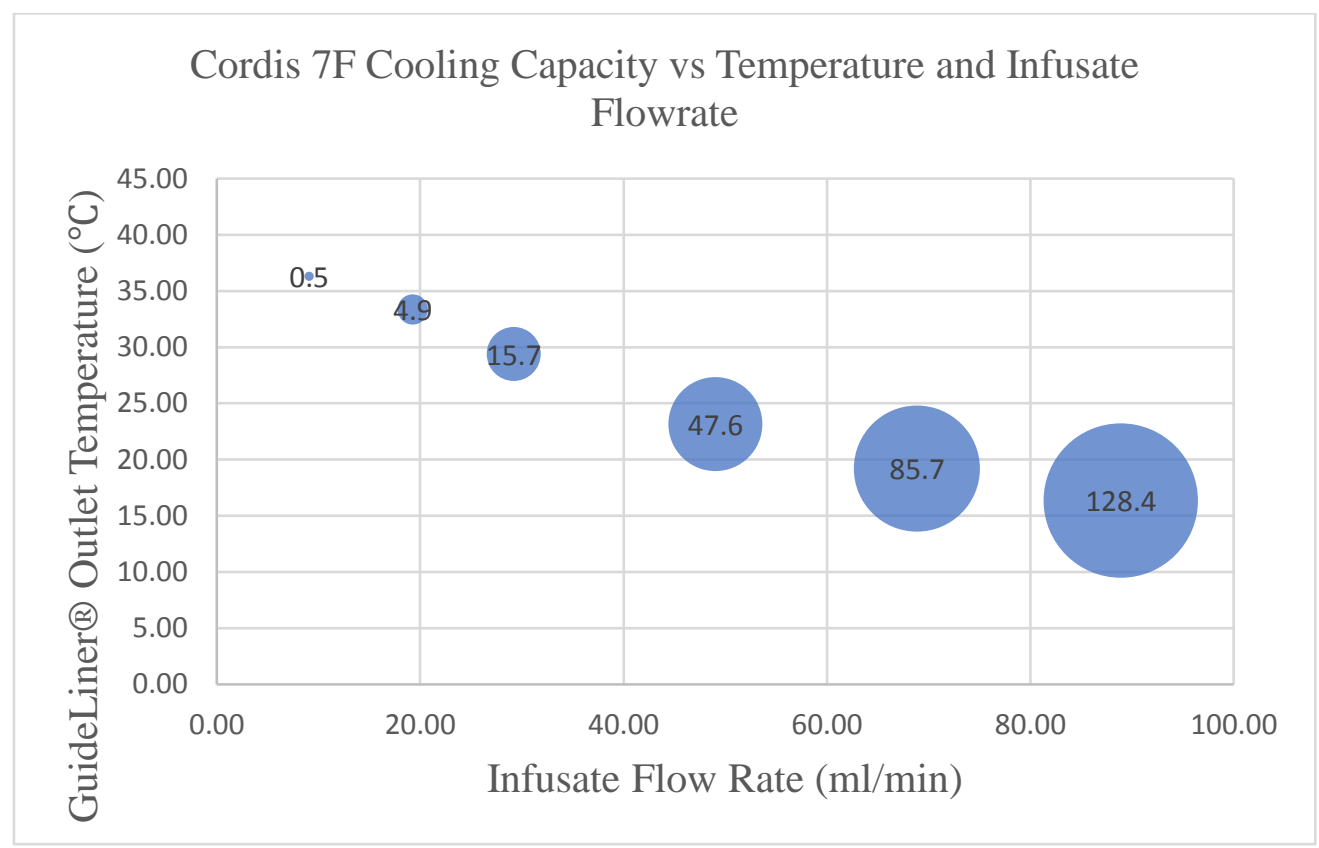

Figure 4.8. Cordis ${ }^{\circledR} 7 \mathrm{~F}$ guide cooling capacity for flowrate vs outlet temperature. Blue circles represent the power $(\mathrm{W})$ required to produce guide extension outlet temperatures at corresponding flowrates. 


\section{Summary and Connection to Computational Work}

In summary, the distilled water exiting the guide extension resulted in

temperatures between $36.31^{\circ} \mathrm{C}$ and $13.36^{\circ} \mathrm{C}$ and volumetric flowrates ranging between $9.08 \mathrm{ml} / \mathrm{min}$ and $88.9 \mathrm{ml} / \mathrm{min}$, respectively.

The minimum power required to cool infusate fluid to $\mathrm{TH}$ temperatures required a minimum of 15.72 Watts, which yielded an outlet temperture of $29.4^{0} \mathrm{C}$. Although six flowrates were analyed and tested in the mock loop system, only three were chosen for the CFD simulations. The first three flowrates that exhibited TH temperatures were chosen for the CFD simulations; $29.2 \mathrm{ml} / \mathrm{min}, 49.03 \mathrm{ml} / \mathrm{min}$, and $68.85 \mathrm{ml} / \mathrm{min}$.

The outlet temperatures and flow rates attained from this study were used as the guide extension outlet conditions in the CFD modeling studies for all representative heart (RH) models. The vascular differences between these RH models are discussed in detail in Chapter 5. 


\section{Chapter 5}

\section{Representative Hearts (RH's)}

\section{Source of Human Heart 3D Models}

In 1997, the Visible Heart® Project was launched as a subsidiary medical division to the University of Minnesota. In 2000, the free-access website, Atlas of Human Cardiac Anatomy was launched from Visible Heart@ to provide free images, videos, and CT/MRI DICOM files to students, academia, and industry personnel [135]. As of 2016, Visible Heart® has reanimated 75 human hearts and its Atlas website contains human cardiac anatomy images from more than 400 human heart specimens. With permission from a donor's family, LifeSource, a non-profit organ donation company, donates hearts from organ donors that have been considered non-viable for transplantation to the Visible Heart@ Laboratory (VHL) [135]. Computed tomography (CT) and magnetic resonance (MR) imaging of the donated hearts are performed internally and uploaded in DICOM format to the Atlas website.

Each human heart $(\mathrm{HH})$ is numbered on the Atlas website and contains detailed descriptions for each specimen's gender, age, and cardiac medical history. VHL was an excellent resource for attaining our high quality cardiac images that can also be used to help guide the verification and development process of new therapeutic hypothermia (TH) transcatheter therapies.

\section{Rationale for selecting specific RH's}

The complex structure of cardiac anatomy plays a vital role in cardiac medical device design and procedures [136][137]. Understanding anatomical variations in human hearts is a critical factor for determining its impact on cardiovascular interventions [138][139][140]. To better understand anatomical variations in left main coronary artery (LMCA) networks and their impact on cardiovascular procedures, a collection of $\mathrm{HH}$ 
models were strategically selected for this study to represent a unique set of similar patient-specific LMCA networks.

The rationale for choosing similar heart models was to apply "identical" CFD boundary conditions and physics solvers to analyze the impact of each patient's coronary structural variations on heart cooling. Human hearts in the VHL database that expressed similarities in LMCA anatomical networks were chosen for this work. The similarities between each model were identified visually based on their vascular network and included similarities in artery network patterns, vessel diameters, and vessel lengths. Choosing hearts with large proximal left anterior descending (LAD) and left circumflex (LCX) artery diameters was critical for accommodating the insertion of a GuideLiner® (Fig. 5.1) in each proximal LAD and LCX artery.

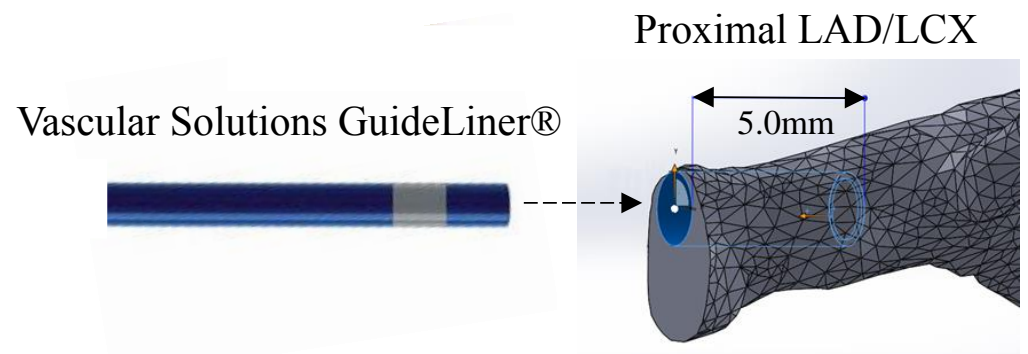

Figure 5.1. Proximal LAD and LCX GuideLiner® insertion method. Large proximal artery diameters are critical for accommodating and simulating GuideLiner ${ }^{\circledR}$ insertion.

For this study, we analyzed 33 patient-specific human hearts from the VHL database. After careful consideration, we chose six human hearts that have no abnormalities and most closely resemble normal LMCA geometries and anatomical configurations. Each heart model (Fig. 5.2) contains varying branch lengths, vessel diameters, and quantity of bifurcated branches in its network. 

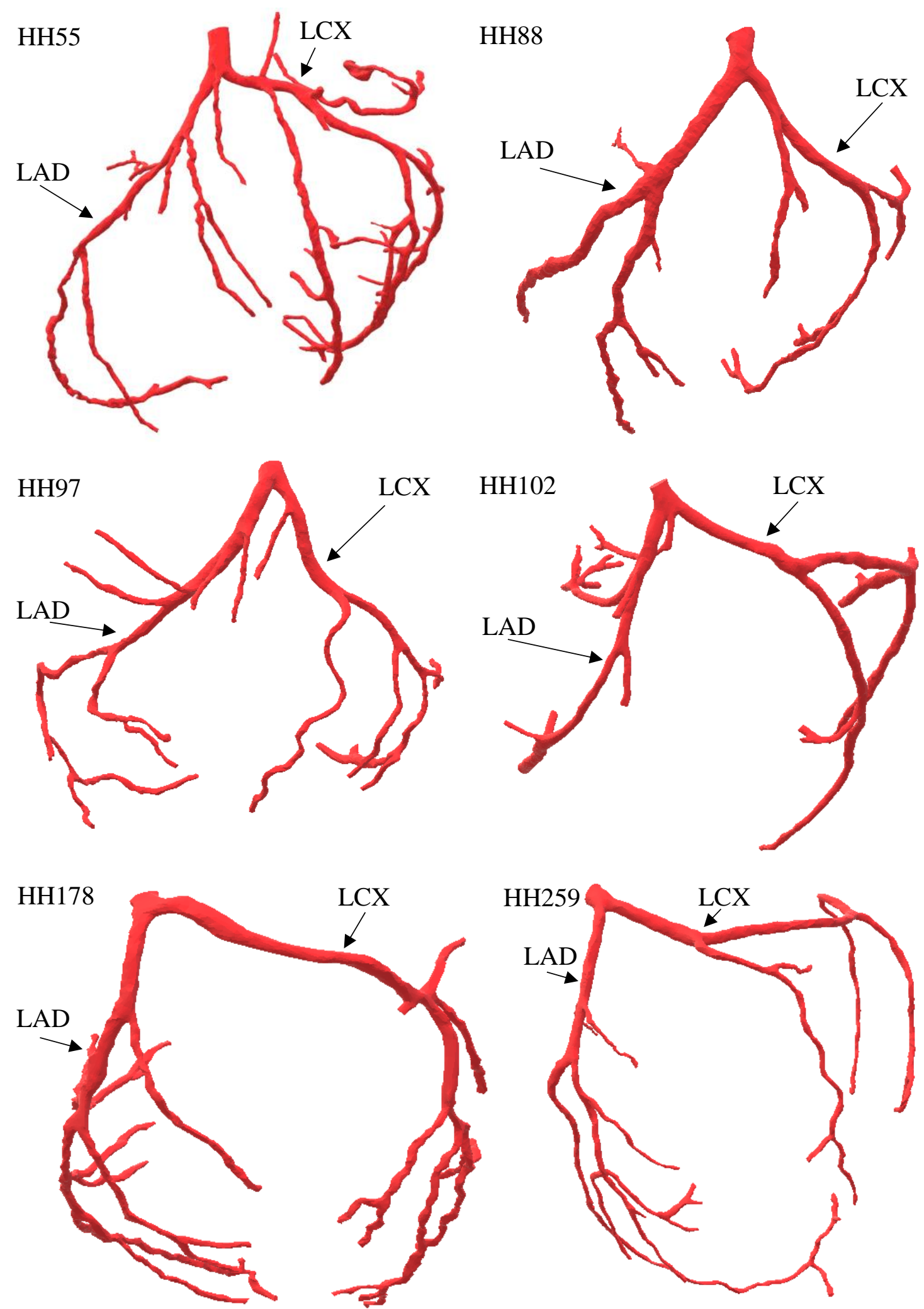

Figure 5.2. Representative hearts (RH) that were chosen for this work. These RH's were created in Mimics ${ }^{\circledR}$ and were exported into STAR-CCM+ for CFD modeling. All RH models contain a LMCA stem that bifurcates into an LAD and LCX artery. 


\section{Heart Model Structural Comparison}

Each heart model expressed vascular differences that varied in artery lengths, diameters, and the number and location of bifurcations stemming from each artery segment. Although 6 patient-specific hearts were chosen for this study, no two hearts were anatomically identical.

Quantify differences between models. The aim of this section is to interpret the vascular differences between all heart models and quantify these differences in terms of length, area, and surface area to volume ratios. The goal is to link these quantitative characteristics to temperature distribution data for each heart model so that assumptions can be made regarding the impact of heart structure on cooling effectiveness.

Lengths. The proximal LAD and LCX is defined as the distance from its left main bifurcation to the first major septal perforator [136][137][138][139][140], and the distance from its left main bifurcation to the first obtuse marginal branch [6][8], respectively.

The segment lengths of the LAD and LCX arteries varied with each HH model. The length and average diameter for each proximal LAD and LCX model was measured and recorded in Table 5.1.

Table 5.1

Length of proximal LAD and LCX arteries before major bifurcation.

\begin{tabular}{|c|c|c|c|c|c|}
$\begin{array}{c}\text { Heart } \\
\text { Model }\end{array}$ & Units & Length & $\begin{array}{c}\text { PVG } \\
\text { Diam. }\end{array}$ & Length & $\begin{array}{c}\text { AVG } \\
\text { Diam. }\end{array}$ \\
\hline HH55 & $\mathrm{mm}$ & 19.8 & 3.14 & 12.9 & 2.79 \\
\hline HH88 & $\mathrm{mm}$ & 31.7 & 4.12 & 12.4 & 3.55 \\
\hline HH97 & $\mathrm{mm}$ & 15 & 3.55 & 9.23 & 2.78 \\
\hline HH102 & $\mathrm{mm}$ & 12 & 2.91 & 28 & 2.44 \\
\hline HH178 & $\mathrm{mm}$ & 22.5 & 3.03 & 57.5 & 2.72 \\
\hline HH259 & $\mathrm{mm}$ & 25 & 3.08 & 29 & 3.45 \\
\hline
\end{tabular}


As seen in Table 5.1, HH102 had the shortest proximal LAD segment (12mm), while HH88 had the longest proximal segment (31.7mm). Consequently, HH97 had the shortest proximal LCX $(9.23 \mathrm{~mm})$ and HH178 had the longest proximal LCX $(57.5 \mathrm{~mm})$. From these observations, LAD and LCX artery geometries and their anatomical network structures are independent of each other (Fig. 5.3) and will need to be evaluated as discrete LAD and LCX models.

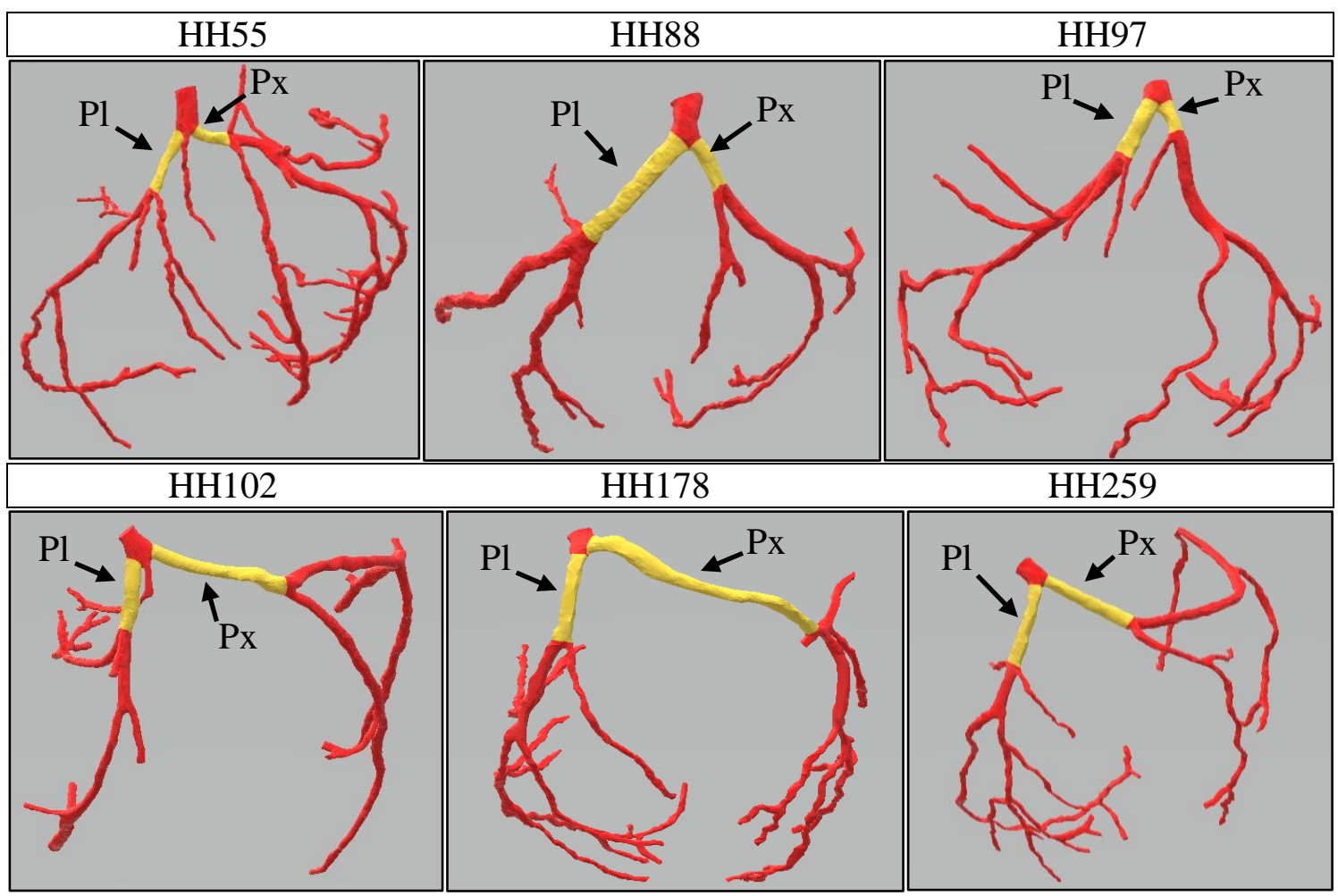

Figure 5.3. The proximal segments of the LAD and LCX arteries highlighted in yellow. Note: proximal $\mathrm{LAD}=\mathrm{Pl}$ and proximal $\mathrm{LCX}=\mathrm{Px}$.

A comparison between the first obtuse marginal branches of the LCX for HH88, HH178, and HH259 (Fig. 5.4) was made to compare the varying lengths of each proximal segment. 

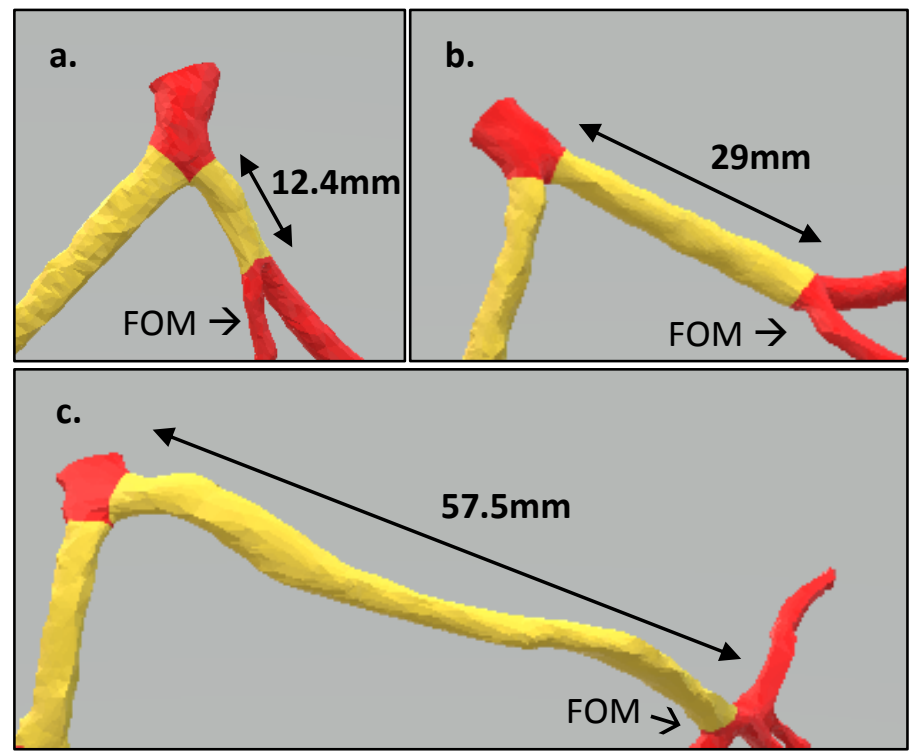

Figure 5.4. Variations in proximal LCX artery lengths. The proximal LCX extends from the left main bifurcation to the first obtuse marginal branch (FOM). HH88 (a) contains the shortest proximal segment length, while HH259 (b) and HH178 (c) contain the average and longest proximal segment lengths of all heart models chosen for this work, respectively.

As described in Fig. 5.4, HH259's proximal trunk is 124\% longer than HH88's, while HH178's proximal trunk is $363 \%$ longer than HH88. This further supports the claim that no two patient-specific heart structures are alike, and that the total lengths of each artery network (Table 5.2) will need to be considered for evaluating TH temperature distributions in CFD post-processing.

Table 5.2

Total length of all vessels in each LAD and LCX structure.

\begin{tabular}{c|ccc}
\multicolumn{1}{c}{ Heart Model } & Units & $\begin{array}{c}\text { Total LAD Vessel } \\
\text { Lengths }\end{array}$ & $\begin{array}{c}\text { Total LCX Vessel } \\
\text { Lengths }\end{array}$ \\
\hline HH55 & $\mathrm{mm}$ & 433.03 & 465.5 \\
HH88 & $\mathrm{mm}$ & 297.7 & 316 \\
HH97 & $\mathrm{mm}$ & 372.9 & 269.03 \\
HH102 & $\mathrm{mm}$ & 157 & 286 \\
HH178 & $\mathrm{mm}$ & 394.3 & 359.13 \\
HH259 & $\mathrm{mm}$ & 429.7 & 373.1
\end{tabular}


Areas. The surface areas for each heart model were generated in SolidWorks using the Mass Properties function. Surface areas are listed for each patient-specific LAD and LCX model in Table 5.3.

Table 5.3

Overall surface area for each $\mathrm{HH}$ model.

\begin{tabular}{c|ccc} 
Human Heart $(\mathbf{H H})$ & Units & LAD Surface Area & LCX Surface Area \\
\hline HH55 & $\mathrm{mm}^{2}$ & 2404.87 & 4105.07 \\
HH88 & $\mathrm{mm}^{2}$ & 2177.65 & 1752.51 \\
HH97 & $\mathrm{mm}^{2}$ & 1913.46 & 1590.99 \\
HH102 & $\mathrm{mm}^{2}$ & 1030.87 & 1613.86 \\
HH178 & $\mathrm{mm}^{2}$ & 2377.44 & 1937.45 \\
HH259 & $\mathrm{mm}^{2}$ & 2227.59 & 2215.9
\end{tabular}

The smallest LAD artery surface area was HH102. HH55's LAD and LCX arteries both dominated in surface area compared to the rest of the heart models, resulting in $2404.87 \mathrm{~mm}^{2}$ and $4105.07 \mathrm{~mm}^{2}$, respectively. The surface area for each LAD and LCX heart model was described by a quantitative plot (Fig 5.5) so that a comparison between surface areas could be made for all RHs. 


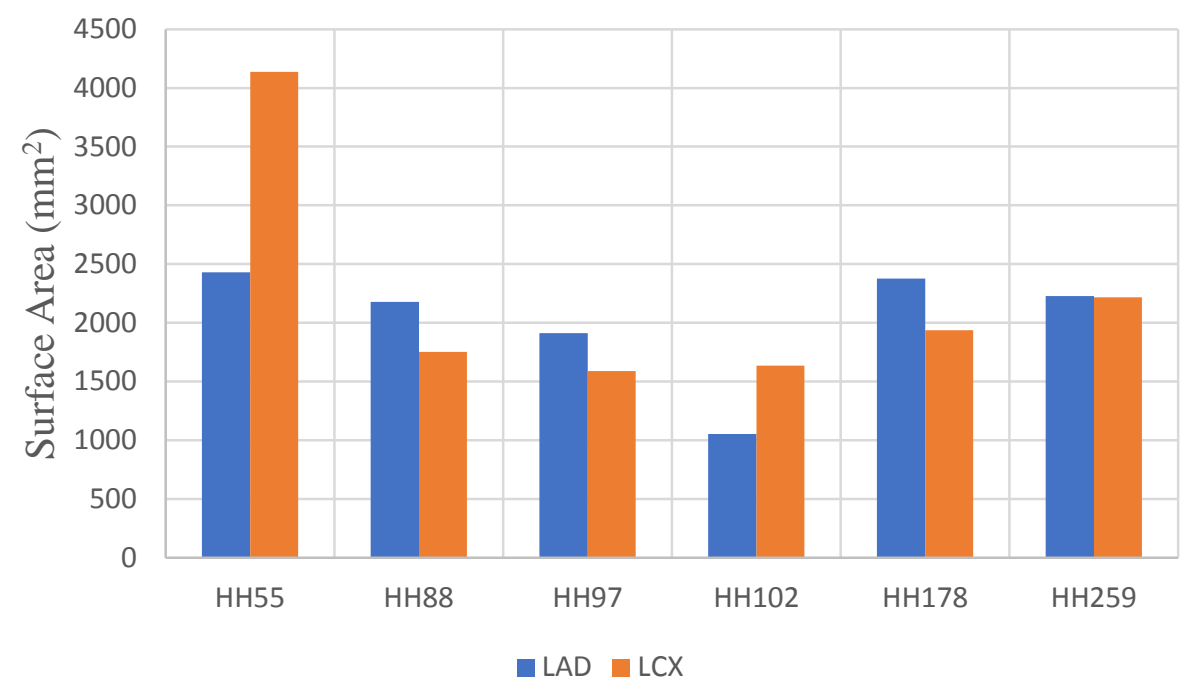

Figure 5.5. Quantitative plot showing the comparison between surface area and all LAD and LCX representative heart $(\mathrm{RH})$ models.

Volumes. Like the surface area comparison in Fig. 5.5, a volume comparison between all LAD and LCX RH models were plotted together (Fig. 5.6) so that a quantitative comparison could be observed.

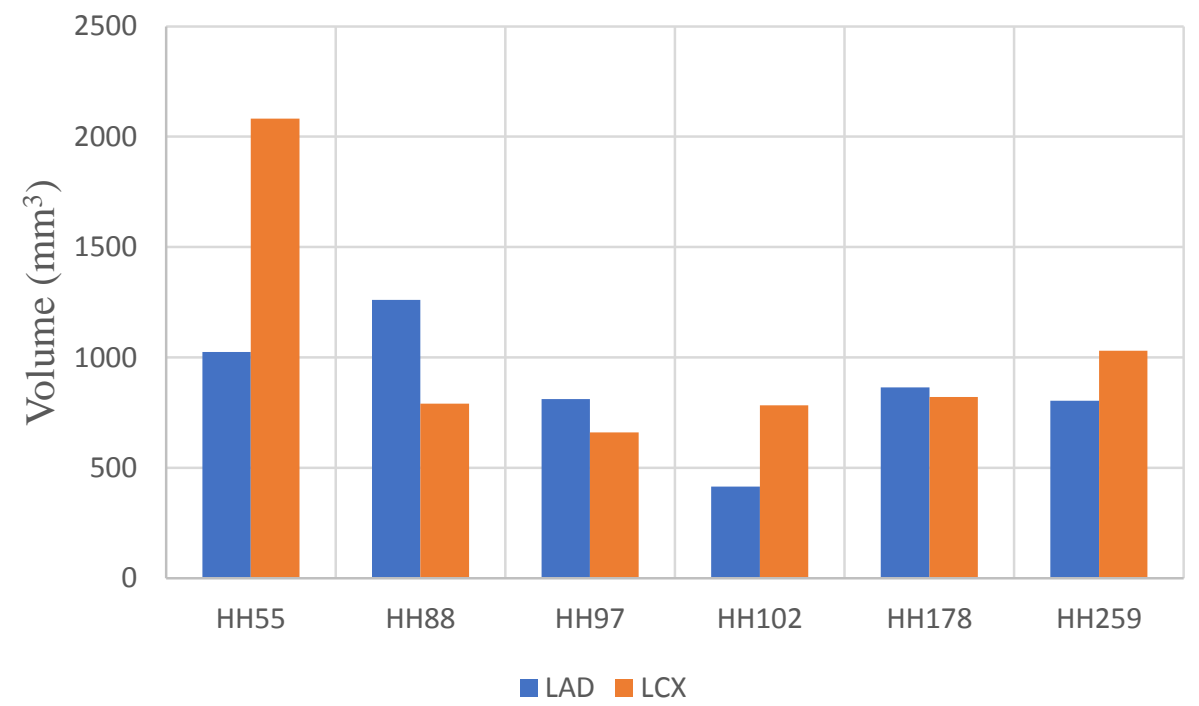

Figure 5.6. Quantitative plot showing the comparison between volume and all LAD and LCX representative heart $(\mathrm{RH})$ models. 
Surface area to volume ratios. The surface area to volume ratios for the LAD and LCX models compare the size of each artery's surface area to the volume inside each artery's network. Surface area to volume ratios (SA:V) were calculated for all LAD and LCX artery models (Table 5.4).

Table 5.4

Surface Area to Volume ratios for each LAD and LCX model employed in the study.

\begin{tabular}{l|ccccccc}
\multicolumn{1}{c}{ HH55 } & HH88 & \multicolumn{1}{c}{ HH97 } & HH102 & HH178 & HH259 & Units \\
\hline LAD & 2.368528 & 1.727352 & 2.35666 & 2.53667 & 2.747695 & 2.767548 & $\mathrm{~m}^{-1}$ \\
LCX & 1.986149 & 2.218433 & 2.406192 & 2.087923 & 2.35993 & 2.152103 & $\mathrm{~m}^{-1}$
\end{tabular}

The surface area and volume of each representative heart model was calculated using the Mass Properties tool in SolidWorks®. The accuracy of each SA:V ratio could be impacted by the guide extension insertion depth in each proximal artery, which ranges from 3-5mm. However, SA:V ratio accuracy could be improved if higher resolution DICOM images were created in Mimics. This would allow us to locate more bifurcations and extend each vessel down to the capillaries (or as close as possible).

High SA:V ratios are typically a result of low volume objects that exhibit large surface areas [141], which could indicate long, thin artery networks in our HH models (Fig. 5.7). 


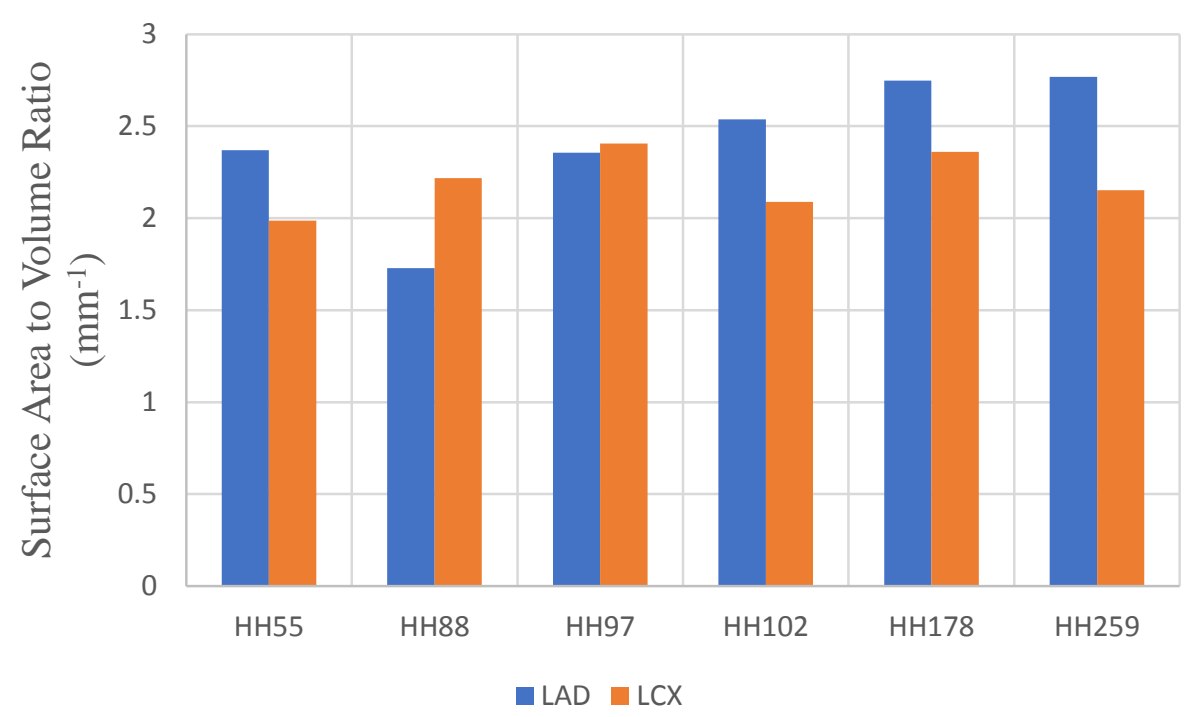

Figure 5.7. Surface area to volume ratios between all LAD and LCX representative heart (RH) models.

Suppose the volume (amount of space) inside an artery network were to grow. The surface area surrounding the volume of this artery network would increase as well [142]. Surface area to volume (SA:V) ratios in cylinders can be simplified mathematically to $2 / \mathrm{R}$, where $\mathrm{R}$ is the radius of the cylinder. Therefore, when volume increases, the SA:V ratio decreases, and vice versa.

Fig. 5.6 shows a visual representation for each heart model and its corresponding surface area to volume ratio. $\mathrm{HH} 259$ has almost identical surface area values for both its LAD and LCX arteries, however, since its LAD has a slightly lower volume to that of its LCX, the surface area to volume ratio is larger for its LAD network. This suggests the possibility that the LAD for HH259 has smaller and longer vessels than its LCX counterpart network.

The rationale for choosing the $\mathrm{RH}$ models in this study was to execute identical interventional CFD simulations and compare the outcomes of all models to reveal the 
impact of vascular structure on cooling impact. Human heart artery lengths, diameters, and SA:V ratios will be used to explore CFD simulation results in succeeding chapters. 


\section{Chapter 6}

\section{Thermal Fluid Model Setup for RHs}

Now that we have established the biophysics background (Ch. 2), described the computational methods (Ch. 3), and introduced the representative heart models (Ch. 5), we will now describe the steps required to solve thermal fluid flow through each of the left main coronary artery (LMCA) networks.

\section{Geometries}

Patient-specific left main coronary artery (LMCA) geometries impact medical device performance. We characterized each artery's network by identifying four main branches in the left anterior descending (LAD) artery, and three main branches in the left circumflex (LCX) artery (Fig. 6.1).

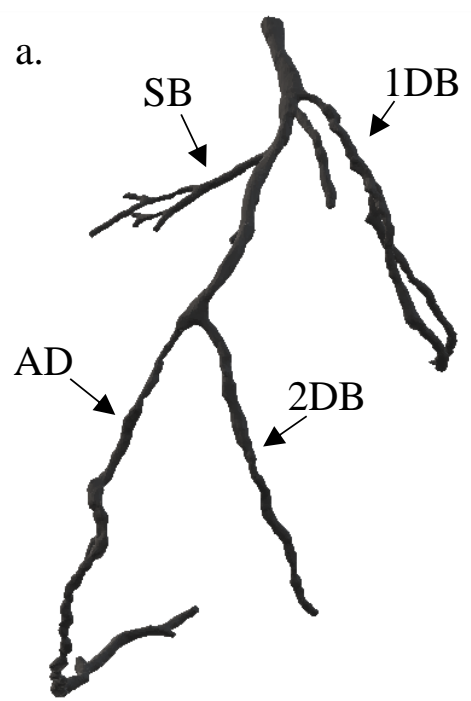

b.

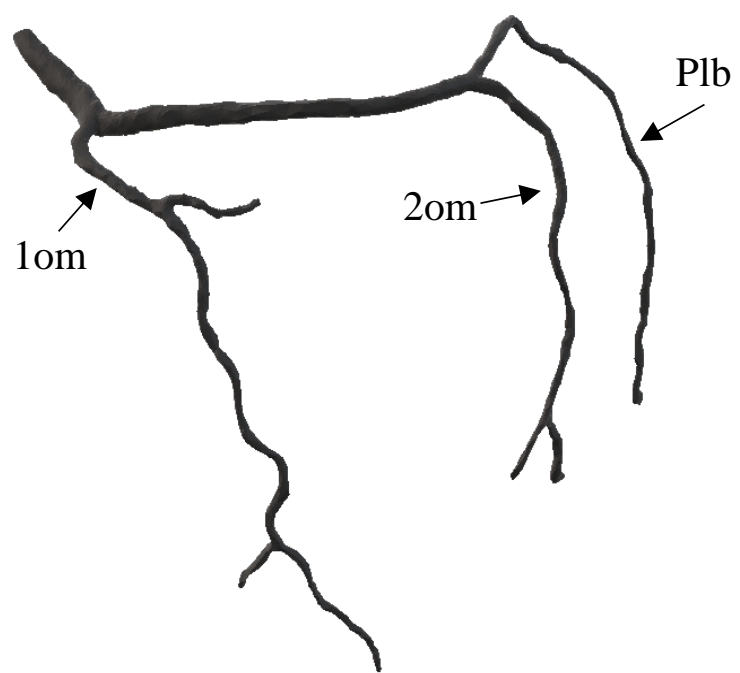

Figure 6.1. Ideal LAD (a) and LCX (b) networks [143]. Note for the LAD (a): first diagonal branch (1DB), second diagonal branch (2DB), septal branches (SB), and anterior descending (AD). Note for the LCX: first obtuse marginal branch (1om), second obtuse marginal branch (2om), and posterolateral branch (Plb). 
We identified four main branches that characterized each LAD model [143]; first diagonal branch, second diagonal branch, septal branches, and the anterior descending branch. Similarly, we identified three main branches that characterized each LCX model [143]; first obtuse marginal and second obtuse marginal branches, and the posterolateral branch. Lastly, it was important to understand the geometry of the 7F Cordis ${ }^{\circledR}$ guide catheter and GuideLiner ${ }^{\circledR}$ used in the in vitro mock loop system. The OD and ID of the GuideLiner® tip (Fig. 6.2) was implemented as a modeling parameter in the proximal LAD and LCX arteries.

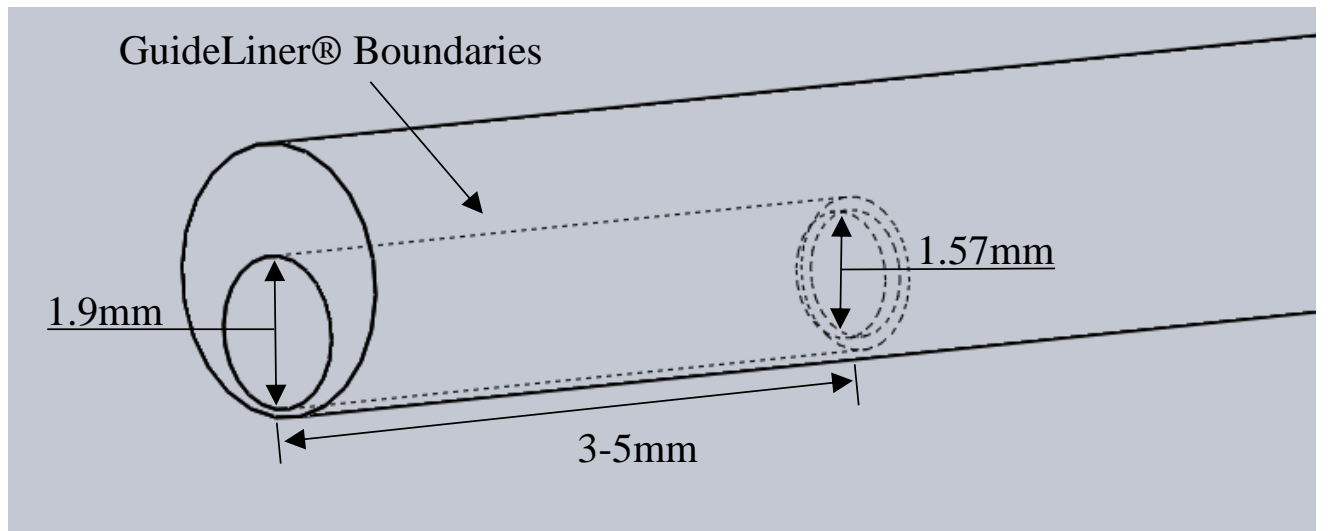

Figure 6.2. Isometric view of a general representation of the OD and ID of the GuideLiner ${ }^{\circledR}$ in a straight tube describing the GuideLiner ${ }^{\circledR}$ boundaries for each proximal artery. The depth of the insertion varied between 3-5mm and the OD and ID were fixed at $1.9 \mathrm{~mm}$ and $1.57 \mathrm{~mm}$, respectively.

Physiological geometry. Each of the six heart models that were chosen for this study were broken down into the LAD and LCX artery networks. The LAD (Figure 6.3) and LCX (Figure 6.4) networks of each patient-specific heart were grouped together so that similarities and differences could be identified between the two groups. 


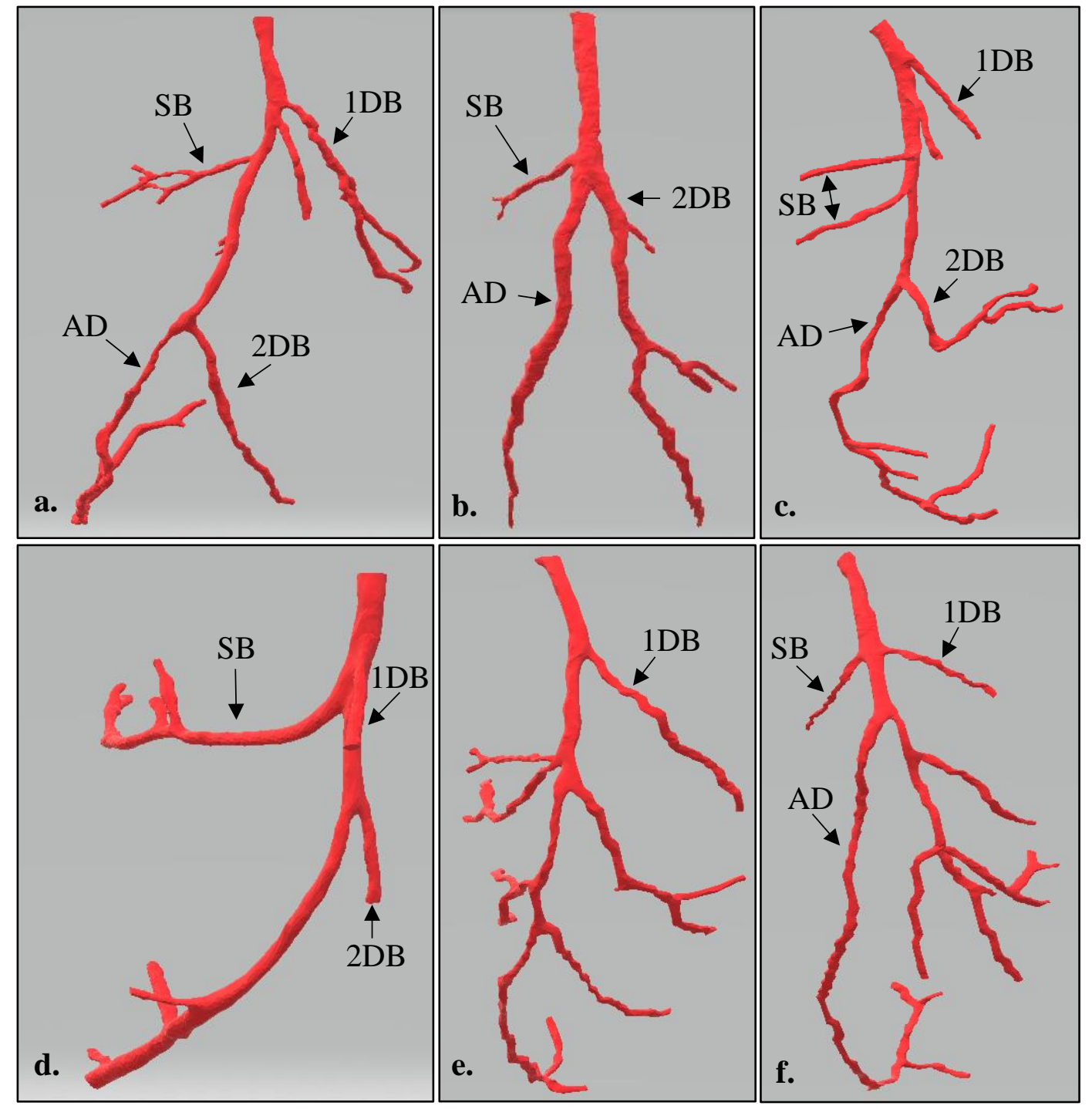

Figure 6.3. Patient-specific LAD networks for HH55, HH88, HH97, HH102, HH178, and $\mathrm{HH} 259$, for hearts a. through f., respectively. Note: first diagonal branch $=1 \mathrm{DB}$, second diagonal branch $=2 \mathrm{DB}$, septal branches $=\mathrm{SB}$, and the anterior descending branch $=\mathrm{AD}$. HH88 had no identifiable septal perforator branch (b), which could have been attributed to poor image quality in the DICOM file format. 


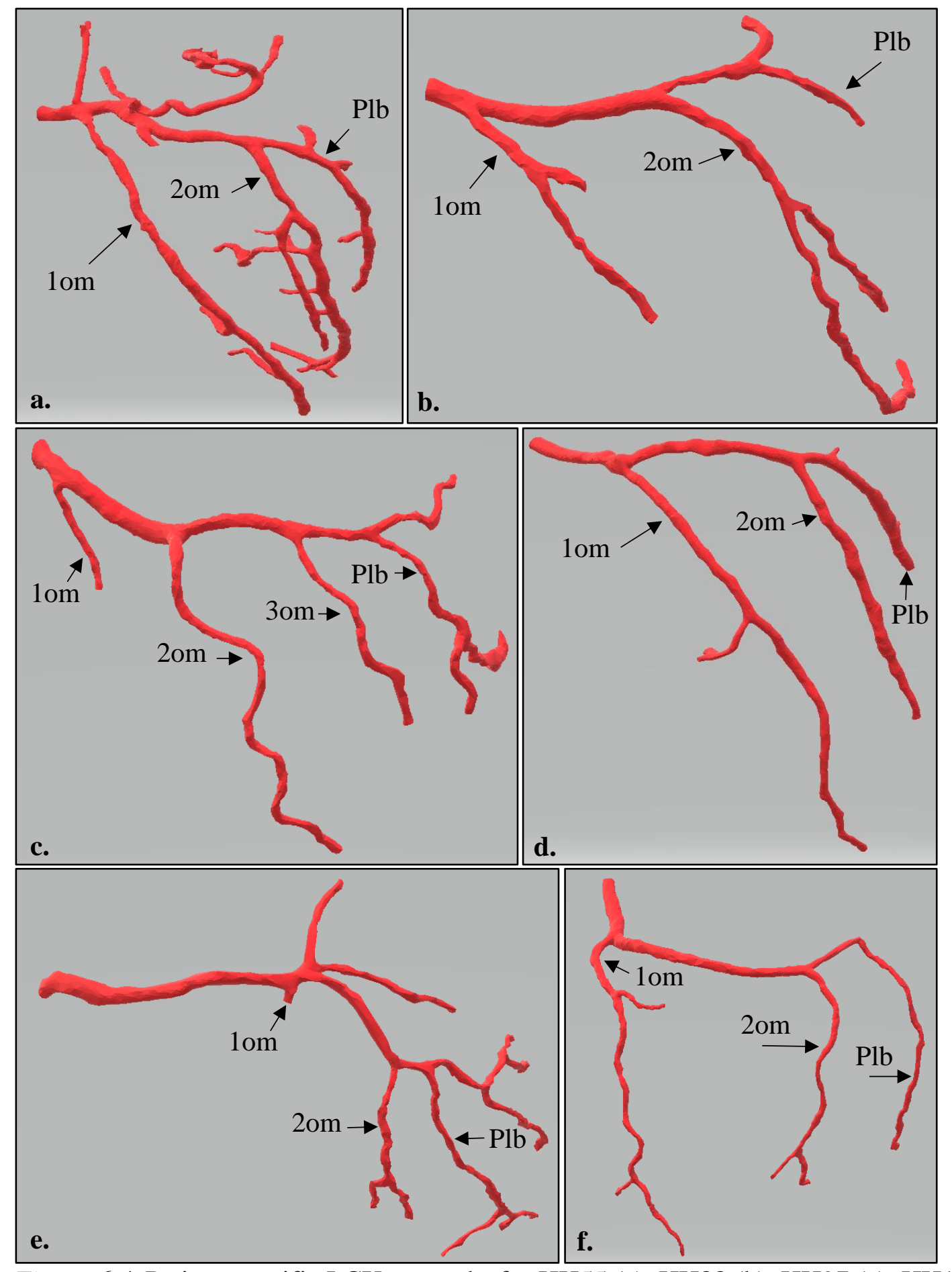

Figure 6.4. Patient-specific LCX networks for HH55 (a), HH88 (b), HH97 (c), HH102 (d), HH178 (e), and HH259 (f). Note: the first obtuse marginal branch = 1om, second obtuse marginal $=2 \mathrm{om}$, and the posterolateral branch $=$ Plb. HH97 appears to have a third obtuse marginal branch (c) that extends between the second obtuse marginal and the posterolateral branches [144]. 
It is important to acknowledge that not all vascular features can be easily resolved. Heart model HH88 LAD had no identifiable septal perforator branch, which could be a result of 1) poor DICOM image quality that was not able to recognize the bifurcation during Mimics ${ }^{\circledR}$ segmentation, or 2) VHL's CT angiogram imaging method did not fully capture the septal perforator branch in the DICOM file. The remaining HH models had identifiable branches that enabled us to characterize the anatomical differences between each model.

Medical device geometry. Both the 7F guiding catheter and the 7F GuideLiner ${ }^{\circledR}$ were used in the mock loop system. To insert these devices into the circulatory mock loop system (Ch. 4), a guide wire was inserted into the proximal hub of the mock loop's femoral artery and advanced through the aorta, where it was steered and exited the left main coronary artery (LMCA). Next, the guide catheter was inserted over the guide wire and advanced to the ostia of the LMCA. The guide catheter was fixed at the LMCA ostium and a GuideLiner® was inserted through the guide catheter and advanced $8.5 \mathrm{~cm}$ out of the catheter outlet.

Although both devices were used in the mock loop study, only 3-5mm of the GuideLiner® tip was modeled and used in the CFD simulations. A description and list of dimensions for each device are listed in Table 6.1.

Table 6.1

Descriptive parameters for each medical device used in the mock loop system.

\begin{tabular}{|cccc|}
\hline Device & Location & Value & Device Length \\
\hline 7F Guiding Catheter (0.078” & ID & $1.98 \mathrm{~mm}$ & $100 \mathrm{~cm}$ long \\
\cline { 2 - 3 } ID) & OD & $2.3 \mathrm{~mm}$ & \\
\hline 7F GuideLiner ${ }^{\circledR}$ V3 Catheter & ID & $1.57 \mathrm{~mm}$ & \multirow{2}{*}{$150 \mathrm{~cm}$ long } \\
\cline { 2 - 3 } & OD & $1.9 \mathrm{~mm}$ & \\
\hline
\end{tabular}


As seen in Table 6.1, the GuideLiner® is $50 \%$ longer than the guide catheter so that it can be inserted and advanced out of the guide catheter. Clearance between the GuideLiner® OD wall and the guide catheter ID wall was $0.08 \mathrm{~mm}$.

To make the inlet geometry as reasonable as possible, we chose to implement an eccentric inlet geometry for each HH model. Since eccentric GuideLiner ${ }^{\circledR}$ placements were evident in the mock circulatory loop (Ch. 4), we can assume that the same phenomenon holds true in a clinical setting. Therefore, to retain consistency, an eccentric GuideLiner@ insertion was assumed lining the anterior side of the coronary artery for all CFD simulations. A general representation for the GuideLiner® inlet geometry in the proximal LAD and LCX (Fig. 6.5) vascular network.

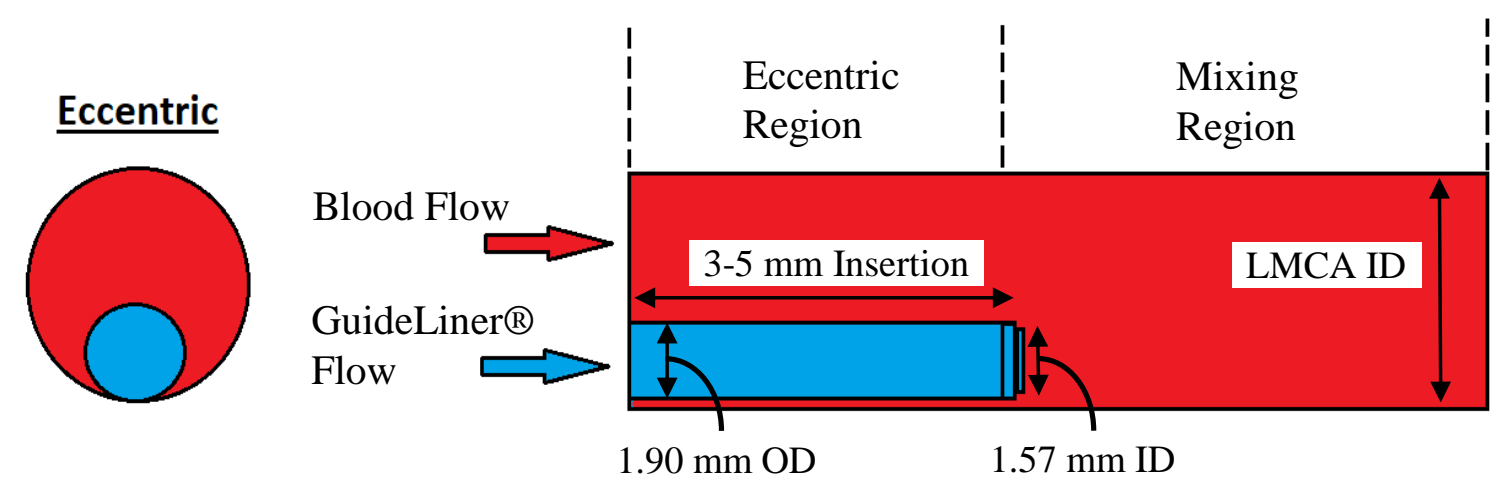

Figure 6.5. Sagittal view of a general representation of the inlet geometry and boundaries describing the GuideLiner ${ }^{\circledR}$ placed in each proximal LMCA. The depth of the insertion varied between $3-5 \mathrm{~mm}$ and the OD and ID were fixed at $1.9 \mathrm{~mm}$ and $1.57 \mathrm{~mm}$, respectively.

It would be unreasonable to assume that physicians achieve consistent eccentric placement of the GuideLiner@ in each proximal LAD and LCX artery in a clinical setting. If however, perfect concentricity could be achieved during intracoronary operations, such that the GuideLiner ${ }^{\circledR}$ could be place at a non-normal angle with respect 
to the arterial cross-section, then temperature distribution results would undeniably be changed. Therefore, to retain consistency in our CFD simulations, an eccentric GuideLiner® insertion lining the anterior side of the artery was assumed for all CFD cases.

\section{Physics}

Prior to executing a CFD model, physics conditions need to be determined so that STAR-CCM+, the finite volume method solver, can apply the correct governing equations. Table 6.2 describes a list of parameters used for the physics conditions in each of our CFD models.

Table 6.2

Physics conditions used for each CFD heart model in STAR-CCM+.

\begin{tabular}{|c|c|c|}
\hline Parameter & Value & Description \\
\hline Space & Three Dimensional & $\mathrm{x}, \mathrm{y}$, and $\mathrm{z}$ coordinate space \\
\hline Time & Steady & $\begin{array}{l}\mathrm{T}=\text { constant. Results } \\
\text { represent a single point in } \\
\text { time. }\end{array}$ \\
\hline Material & Liquid & --- \\
\hline Flow & Segregated Flow & $\begin{array}{l}\text { Continuity, momentum, and } \\
\text { energy are solved } \\
\text { sequentially (segregated from } \\
\text { each other). }\end{array}$ \\
\hline Equation of State & Constant Density & Incompressible fluid \\
\hline Viscous Regime & Laminar & $\operatorname{Re}<2300$ \\
\hline Solver & Segregated Fluid Temperature & $\begin{array}{l}\text { Entire energy equation is } \\
\text { solved with } \mathrm{T} \text { as independent } \\
\text { variable. Enthalpy is then } \\
\text { calculated from temperature } \\
\text { as stated by the equation of } \\
\text { state. }\end{array}$ \\
\hline
\end{tabular}

Note $. \mathrm{Re}=$ Reynolds number, $\mathrm{T}=$ Temperature. 
Governing equations. As discussed in Chapter 2, the Navier-Stokes equations for conservation of linear momentum (Eq. 2.4a), the continuity equation of conservation of mass (Eq. 2.5), and the conservation of thermal energy (Eq. 2.7), govern the fluid dynamics of flow and thermal energy in a physics domain.

$$
\begin{gathered}
\rho \frac{d V}{d t}=\rho g-\nabla p+\mu \cdot \nabla^{2} V \\
\nabla \cdot V=0 \\
\rho C_{p}\left[u \frac{\partial T}{\partial x}+v \frac{\partial T}{\partial y}+w \frac{\partial T}{\partial z}\right]=k\left[\frac{\partial^{2} T}{\partial x^{2}}\right]+k\left[\frac{\partial^{2} T}{\partial y^{2}}\right]+k\left[\frac{\partial^{2} T}{\partial z^{2}}\right]
\end{gathered}
$$

where Eq. 2.7 is coupled with Eq. 2.4a because the viscosity, $\mu(T)$ is a function of temperature, where $\mathrm{T}$ is determined from Eq. 2.7. The coupling is performed so that viscosity can be solved as a function of temperature, $\mu(T)$.

Boundary conditions. Equations 2.4a and 2.5 are applied as the governing equations necessary to solve fluid pressure and velocity fields in the coronary arteries. To solve these equations for flow field properties, appropriate boundary conditions will need to be fixed. The boundary conditions implemented in each heart simulation are described in Figure 6.6. 


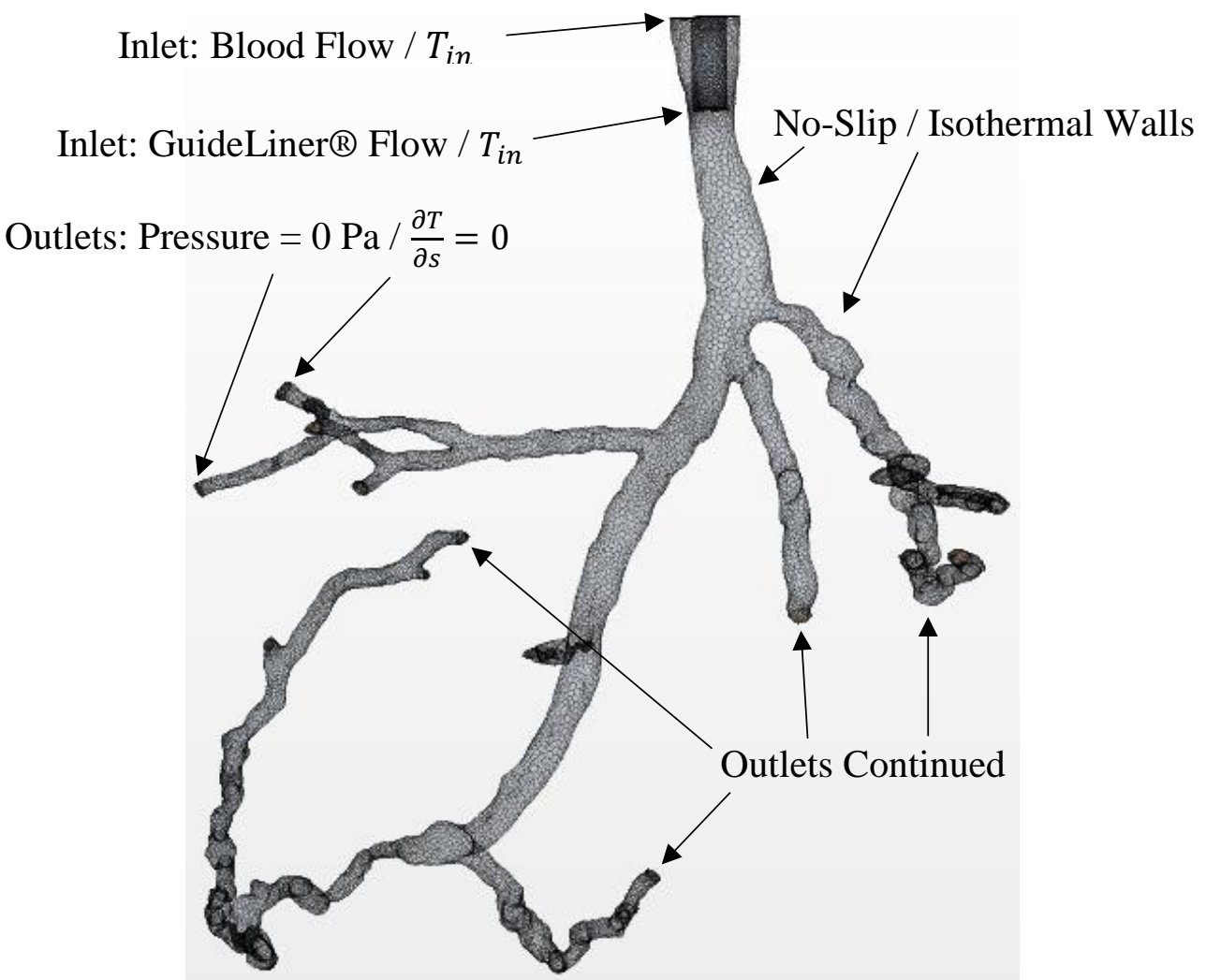

Figure 6.6. HH55 LAD Navier Stokes and thermal energy boundary conditions applied to all patient-specific RH models. Identical boundary conditions were applied to each of the remaining LAD and LCX artery models. Each heart model exhibits a single blood flow inlet and GuideLiner® flow inlet. The number of outlets for each heart model varied and ranged from 7 outlets for HH88 LAD to 33 outlets for HH55 LCX. Navier-Stokes description is designated to left of comment (slash), while thermal energy is represented to the right.

For this study, six patient-specific hearts were chosen and prepared for CFD modeling. The LMCA for each heart was modeled and segmented into six LADs and six LCXs. Each of the 12 patient-specific heart models represent the blood domain of the LAD and LCX and excludes the vascular wall and tissue domain. Each heart model represents patient-specific asymmetric artery networks with rigid, no-slip boundary conditions at the walls, where velocity is zero.

The outlets were assumed to a constant pressure of 0.0 pascal. The inlets used a mass flow inlet condition that represented both the blood flow and chilled GuideLiner® 
flow entering the artery. Although blood flow is pulsatile in nature and GuideLiner® flow is steady, a steady state laminar blood and GuideLiner® flow rate was assumed.

Blood will be considered as an incompressible, Newtonian fluid, as described in Chapter 2. Dynamic viscosity and its shear-thinning correlation will be discussed in greater detail later in this chapter.

To simulate therapeutic hypothermia (TH) in the blood domain, a catheter is typically inserted into the femoral artery and advanced into the coronary ostia. A GuideLiner® is advanced out of the catheter and into the proximal artery (Fig. 6.7) where cooled infusate is delivered and mixed with blood flow.

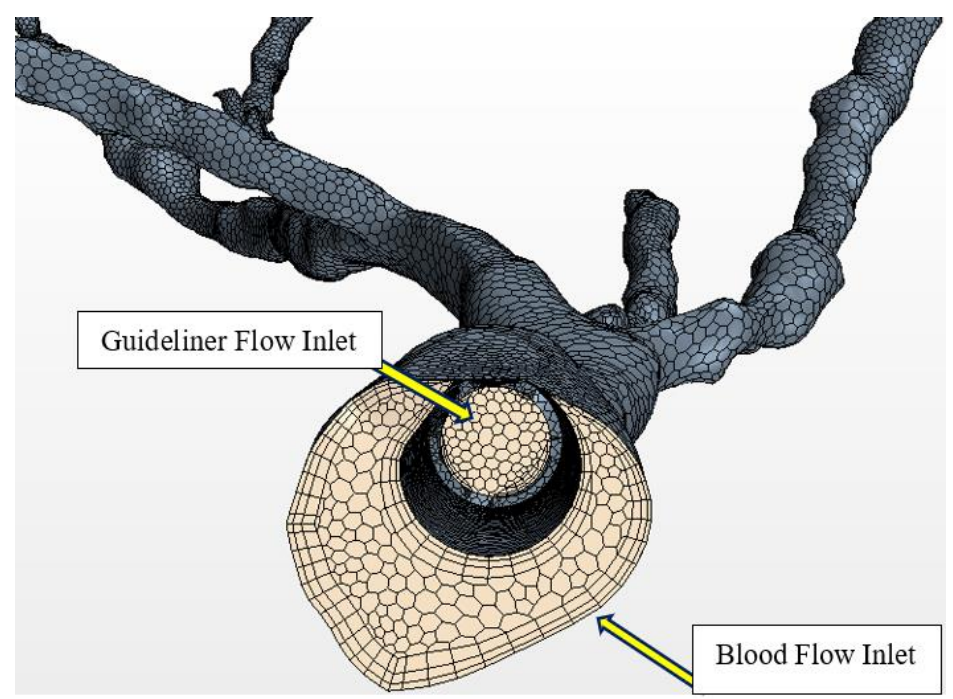

Figure 6.7. Eccentric GuideLiner ${ }^{\circledR}$ catheter in the proximal HH55 LAD representing two distinct inlets for the GuideLiner ${ }^{\circledR}$ and blood flow. Inlet flow conditions for the blood and GuideLiner® lead to assumed complete mixing solution of blood and distilled water.

In vivo blood flow rates entering the proximal LAD and LCX arteries have been determined using methods involving lumped parameter modeling of computed tomography (CT) scans [9], 3-dimensional epicardium simulations [10], ultrasound [11], and phase contrast magnetic resonance imaging (MRI) [12]. As a result, average blood 
flow in the LMCA is estimated between $29.06 \mathrm{~mL} / \mathrm{min}$ and $90.6 \mathrm{~mL} / \mathrm{min}$ [9][12]. Thus, based on these findings, we chose a distinctive total inlet flow rate of $75 \mathrm{~mL} / \mathrm{min}$ (Fig. 6.8) for all of our simulations.

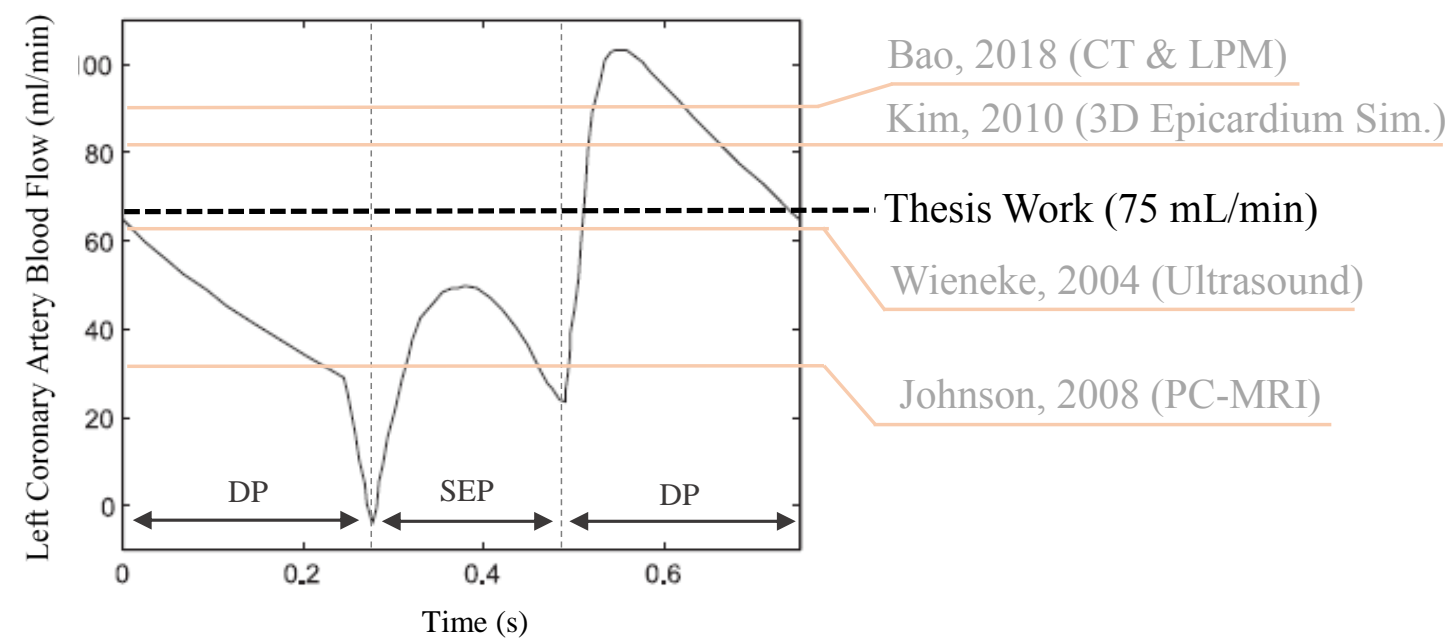

Figure 6.8. Average left coronary artery blood flow as determined in literature using various methods involving lumped parameter modeling, 3D epicardium simulations, ultrasound, and phase contrast MRI. Based on these published findings, a total inlet flow rate of $75 \mathrm{~mL} / \mathrm{min}$ was chosen for all of our CFD simulations.

Where the total inlet flow rate of $75 \mathrm{~mL} / \mathrm{min}$ is independent of blood and GuideLiner® flow rates (Fig. 6.9), and remain a constant parameter for all CFD simulations.

$75 \mathrm{~mL} / \mathrm{min}$ Total Flow

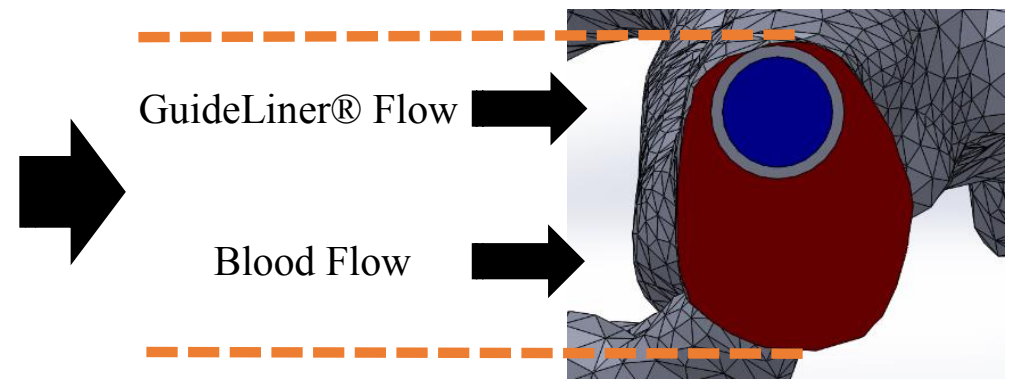

Figure 6.9. Total inlet flow rate representing GuideLiner ${ }^{\circledR}$ and blood flow entering each proximal LAD and LCX artery network. 
Boundary conditions are an important pre-processing step to solving any CFD model. Once boundary conditions are fixed, they govern the domain and boundaries of the model and set the stage for executing the CFD solvers. Table 6.3 lists detailed boundary conditions that were applied to each heart model simulation in STAR-CCM+.

Table 6.3

Boundary conditions and equations applied to each human heart model in STAR-CCM+. The inlet conditions represent the final three flow rates used in the CFD simulations.

\begin{tabular}{c|ccc} 
Boundary Condition & Navier-Stokes & Thermal Energy & References \\
\hline Blood In & $6.15 \mathrm{ml} / \mathrm{min}-$ & $37^{\circ} \mathrm{C}$ & [4], See Chapter \\
& $45.8 \mathrm{ml} / \mathrm{min}$ & & Results \\
GuideLiner@ In & $29.2 \mathrm{ml} / \mathrm{min}-$ & $29.4^{\circ} \mathrm{C}-19.2^{\circ} \mathrm{C}$ & $\begin{array}{c}\text { See Chapter } 4- \\
\text { Results }\end{array}$ \\
& $68.85 \mathrm{ml} / \mathrm{min}$ & & {$[145]$} \\
Out & $\mathrm{P}=0 \mathrm{~Pa}$ & $\frac{\delta \mathrm{T}}{\delta \mathrm{s}}=0$ & {$[13]$}
\end{tabular}

We assume that the rate of change of temperature along the vessels approaches zero at the vascular outlets, therefore $\frac{\delta T}{\delta s}=0$. As the purpose of this study is to examine temperature distributions in patient-specific LAD and LCX arteries, setting the wall temperature to isothermal is an expedient assumption for this study. The expectation of impending tissue cooling studies is that wall-coupled heat transfer exists in real life applications and should be implemented in future studies to improve model fidelity.

\section{Properties}

We used mixture properties of blood and distilled water (infusate), after the two fluids mixed (Fig. 6.10), as inlet conditions for the CFD models. 


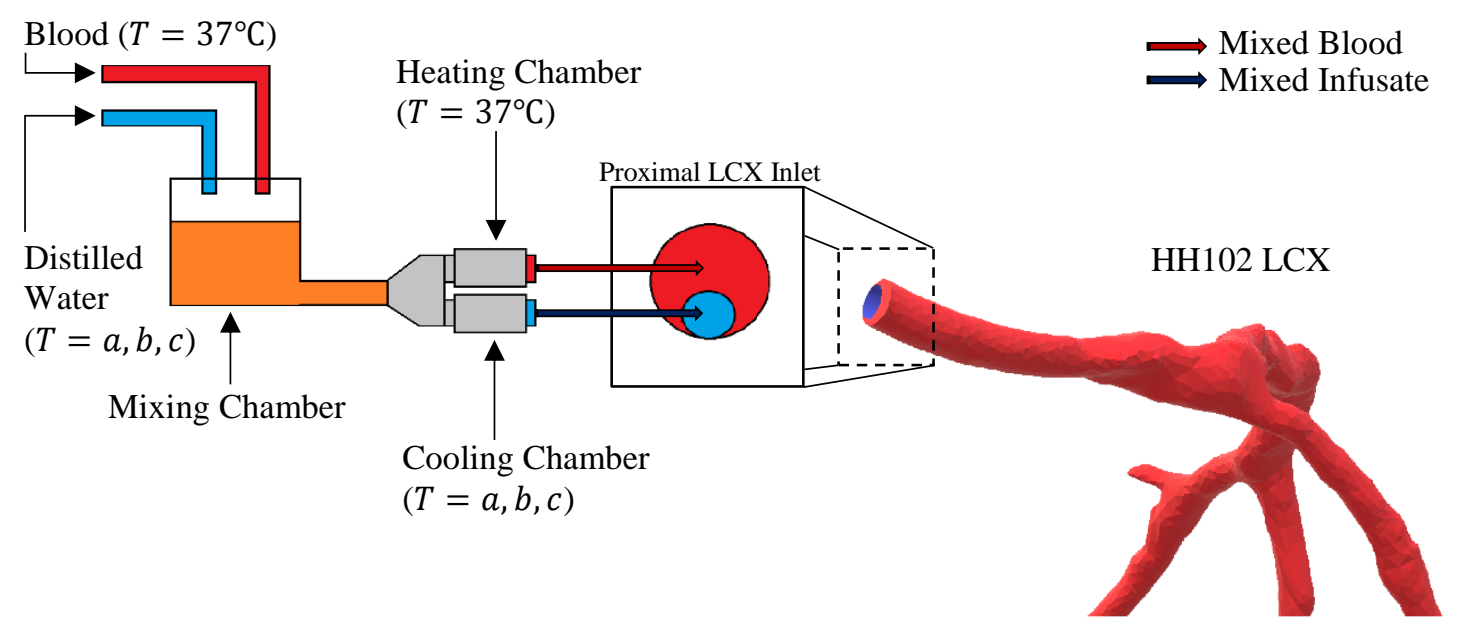

Figure 6.10. Theoretical description of how mixture properties for blood and distilled water were determined for the CFD inlet boundary conditions. $T=a, b, c$ represents the guide extension inlet temperatures $29.4,23.16$, and $19.2^{\circ} \mathrm{C}$ with corresponding flow rates $29.2,49.03,68.85 \mathrm{ml} / \mathrm{min}$, respectively. Blood and distilled water was mixed at $T=$ $a, b, c$ guide extension infusate temperatures, and new mixed blood and infusate properties were used as inlet conditions for the CFD models.

The blood and distilled water flow through the guide extension entering the proximal end of the artery represents a mixture of the two. However, although both fluids have the same properties after mixing, the mixed infusate fluid will be entering the guide extension inlet at temperatures (a, b, c) (Fig. 6.10), while the mixed blood fluid will be entering the blood flow inlet at $37^{\circ} \mathrm{C}$. Since we assume complete mixing happens immediately beyond the inlet, a limitation to this approach will be that the properties down-stream are not experiencing perfect mixing. This will be discussed further in Chapter 7. The three GuideLiner® flowrates and corresponding temperatures used in the CFD simulations were determined from Chapter 4 - In Vitro Methods.

Density. To determine the final mixture property for density after the two fluids mixed, the following linear mixing model (Eq. 6.1) was used:

$$
\rho_{m}=\frac{1}{\dot{m}_{T}}\left(\dot{m}_{I} \rho_{I}+\dot{m}_{B} \rho_{B}\right)
$$


where $\rho_{m}$ is the mixed density $\left(\frac{\mathrm{kg}}{\mathrm{m}^{3}}\right), \dot{m}_{I}$ is the infusate mass flow rate $\left(\frac{\mathrm{kg}}{\mathrm{s}}\right), \dot{m}_{T}$ is the total mass blood flow $\left(\frac{\mathrm{kg}}{\mathrm{s}}\right)$ into the artery, $\dot{m}_{B}$ is the blood mass flow rate $\left(\frac{\mathrm{kg}}{\mathrm{s}}\right)$, and $\rho_{I}$ and $\rho_{B}$ are the infusate and blood density $\left(\frac{\mathrm{kg}}{\mathrm{m}^{3}}\right)$, respectively.

Thermal conductivity. To determine the mixture thermal conductivity after the two fluids mixed, a second linear mixing model (Eq. 6.2) was used:

$$
k_{m}=\frac{1}{\dot{m}_{T}}\left(\dot{m}_{I} k_{I}+\dot{m}_{B} k_{B}\right)
$$

where $k_{m}$ is the mixed thermal conductivity $\left(\frac{W}{m \cdot K}\right)$, and $k_{I}$ and $k_{B}$ is the thermal conductivity of the infusate and blood $\left(\frac{W}{m \cdot K}\right)$, respectively.

Specific heat. To determine the mixture specific heat after the two fluids mixed, Eq. 6.3 was used:

$$
c_{p, m}=\frac{1}{\dot{m}_{T}}\left(\dot{m}_{I} c_{p, I}+\dot{m}_{B} c_{p, B}\right)
$$

where $c_{p, m}$ is the mixed specific heat $\left(\frac{J}{\mathrm{~kg} \cdot \mathrm{K}}\right), c_{p, I}$ and $c_{p, B}$ is the specific heat of the infusate and blood $\left(\frac{\mathrm{J}}{\mathrm{kg} \cdot \mathrm{K}}\right)$, respectively.

To determine the final mixture property for the temperature after the two fluids mixed, the temperature and specific heat for both fluids, as well as all three flowrates employed in the study were required to solve equation Eq. 6.4.

$$
T_{m}=\frac{\left(\dot{m}_{I} \cdot c_{p, I} \cdot T_{I}\right)+\left(\dot{m}_{B} \cdot c_{p, B} \cdot T_{B}\right)}{\dot{m}_{T} \cdot c_{p, m}}
$$

where $T_{m}$ is the mixed temperature $\left({ }^{\circ} \mathrm{C}\right)$, and $T_{I}$ and $T_{B}$ is the temperature of the infusate and blood $\left({ }^{\circ} \mathrm{C}\right)$, respectively. The mixed temperature $T_{m}$ is used as a parameter to solve for the dynamic viscosity. 
Dynamic viscosity. To determine the dynamic viscosity, Eq. 2.11 from Chapter 2 was used to determine the dynamic viscosity as a function of mixed temperature $T_{m}\left({ }^{\circ} \mathrm{C}\right)$, shear rate $\Upsilon\left(\frac{1}{s}\right)$, and hematocrit (Hct $\left.(\%)\right)$ for each infusate flow rate [123].

$$
\mu\left(\Upsilon, \text { Hct, } T_{m}\right)=\left(e^{\frac{\lambda}{\Upsilon+\eta \cdot H c t}}\right)\left[\alpha+\frac{\phi}{\left(1+e^{\beta(T-\epsilon)}\right)}\right]
$$

Therefore, the shear rate, hematocrit, and temperature are independent variables and the dynamic viscosity is the dependent variable for this solution. Please see Appendix B for a description of how viscosity is entered as a function of temperature in STAR$\mathrm{CCM}+$.

Property summary. Key properties are density $(\rho)$, specific heat $\left(c_{p}\right)$, thermal conductivity (k), and dynamic viscosity $(\mu)$, as shown in the governing equations (Eq. 2.11 and Eq. 6.1 to 6.4). The material properties for both blood and distilled water are temperature dependent and are listed in Table 6.4.

Table 6.4

Material properties for blood and distilled water entering the proximal LAD and LCX [146][147][148].

Material Property

Units

Blood

Distilled Water

\begin{tabular}{|c|c|c|c|}
\hline Density $(\boldsymbol{\rho})$ & $\mathrm{kg} / \mathrm{m}^{3}$ & 1060 & 1006 \\
\hline \multirow{2}{*}{ Specific Heat $\left(\boldsymbol{c}_{\boldsymbol{p}}\right)$} & $\mathrm{J} / \mathrm{kg}-\mathrm{K}$ & 3560 & 4180 \\
\hline \multirow{2}{*}{ Thermal Conductivity (k) } & $\mathrm{W} / \mathrm{m}-\mathrm{K}$ & 0.52 & 0.598 to 0.614 \\
& & $\left(\right.$ at $\left.37^{\circ} \mathrm{C}\right)$ & (at $19.2^{\circ} \mathrm{C}$ to $29.4^{\circ} \mathrm{C}$ ) \\
\hline
\end{tabular}

Blood properties were determined from literature [130][140] assuming whole blood, meaning plasma plus red blood cells at $37^{\circ} \mathrm{C}$, while the distilled water properties 
were determined from the in vitro GuideLiner® infusion temperatures ranging between $19.2^{\circ} \mathrm{C}$ and $29.4^{\circ} \mathrm{C}$

The blood and GuideLiner® inlet conditions in each CFD model represent blood properties from literature combined with the in vitro circulatory loop distilled water properties. These two fluids experienced different flow rates and temperatures undergoing developing flow in the proximal artery network. Density and specific heat were assumed to be dependent on the blood and distilled water material property only, rather than temperature, since blood and distilled water were assumed to be mixed having combined thermal-fluid properties. Table 6.5 summarizes the mixed material properties used in our thermal fluid models.

Table 6.5

Blood and distilled water mixed fluid properties at variable GuideLiner ${ }^{\circledR}$ flow rates.

\begin{tabular}{|c|c|c|c|c|}
\hline Material Property & Units & $\begin{array}{l}\text { Mixed } \\
\text { Fluid } 1\end{array}$ & $\begin{array}{l}\text { Mixed } \\
\text { Fluid2 }\end{array}$ & $\begin{array}{l}\text { Mixed } \\
\text { Fluid }_{3}\end{array}$ \\
\hline \multirow{2}{*}{$\begin{array}{c}\text { Density }(\rho) \\
\text { Specific Heat }\left(c_{p}\right)\end{array}$} & $\mathrm{kg} / \mathrm{m}^{3}$ & 1010.43 & 1024.7 & 1038.98 \\
\hline & $\mathrm{J} / \mathrm{kg}-\mathrm{K}$ & 4129.16 & 3965.31 & 3801.39 \\
\hline \multirow{3}{*}{$\begin{array}{c}\text { Thermal Conductivity (k) } \\
\text { Dynamic Viscosity }(\mu) \\
\text { Shear Rate }(\boldsymbol{Y})\end{array}$} & $\mathrm{W} / \mathrm{m}-\mathrm{K}$ & 0.5916 & 0.5723 & 0.5566 \\
\hline & $\mathrm{Pa}-\mathrm{s}$ & 0.00358 & 0.00205 & 0.00180 \\
\hline & $1 / \mathrm{s}$ & \multicolumn{3}{|c|}{2060.66} \\
\hline Mixed Temperature (T) & ${ }^{\circ} \mathrm{C}$ & 20.4584 & 27.4625 & 33.7464 \\
\hline
\end{tabular}

Note. Mixed fluids 1, 2, and 3, correspond to GuideLiner® flow rates $68.85 \mathrm{ml} / \mathrm{min}$, $49.03 \mathrm{ml} / \mathrm{min}$, and $29.2 \mathrm{ml} / \mathrm{min}$, respectively.

The material properties employed in the CFD model, specifically density, specific heat and thermal conductivity, were calculated as a mixture assuming perfect mixing of the blood and distilled water. The resulting mixed fluid properties were established as the primary fluids entering as blood flow and GuideLiner ${ }^{\circledR}$ inlet conditions in each CFD simulation. 
Dynamic viscosity is the only property dependent on temperature, hematocrit, and shear rate, as seen in Eq. 2.11. The remaining mixed properties such as density $\left(\rho_{m}\right)$, specific heat $\left(c_{p, m}\right)$, and thermal conductivity $\left(k_{m}\right)$ are dependent on flow rate, as seen in equations 6.1 to 6.3 . Blood and GuideLiner® inlet temperature conditions were assumed to be mixed (Fig. 6.10) and employed as inlet conditions for each thermal fluid model. Mixed temperature conditions were dependent on specific heat of both fluids, blood flow rate, and each of the three GuideLiner® inlet flow rates. The key property assumptions for each fluid are described in Table 6.6.

Table 6.6

Key properties and assumptions for each mixed fluid.

Property

Density

Specific Heat

Thermal Conductivity

Mixed Temperature

Dynamic Viscosity

\section{Assumption}

Temperature independent \& flow rate dependent Temperature independent \& flow rate dependent

Temperature dependent Flow rate \& specific heat dependent Temperature, Hematocrit, and Shear Rate dependent

In summary, the in vitro mock circulatory loop contained distilled water only. The fluid properties for each corresponding GuideLiner ${ }^{\circledR}$ flow rate were calculated from the in vitro mock circulatory loop and the blood plasma properties were determined from literature (see Table 6.4). The material properties and corresponding assumptions (Table 6.6) for the blood and distilled water were combined to determine mixed fluid properties that were used as inlet conditions in each CFD simulation. 


\section{Post Processing}

Mesh convergence. A mesh convergence analysis, as described in Chapter 3, was performed for each of the $6 \mathrm{LAD}$ and $6 \mathrm{LCX}$ models to determine an appropriate mesh density. The mesh density was determined by setting various base sizes in STAR-CCM+. Base sizes in STAR-CCM+ are values that enable ratios to exist between boundary conditions and the physics solvers. If the base size is a large value, the mesh will be coarse, and the physics solvers will not compute an accurate solution. On the other hand, if the base size is a small value, the mesh will be generated as a fine mesh and will yield a more accurate solution, albeit, with greater computational expense.

For all models, a polyhedral mesh was generated using 3 prism layers (Fig. 6.11), which generated a layered mesh at each wall boundary.

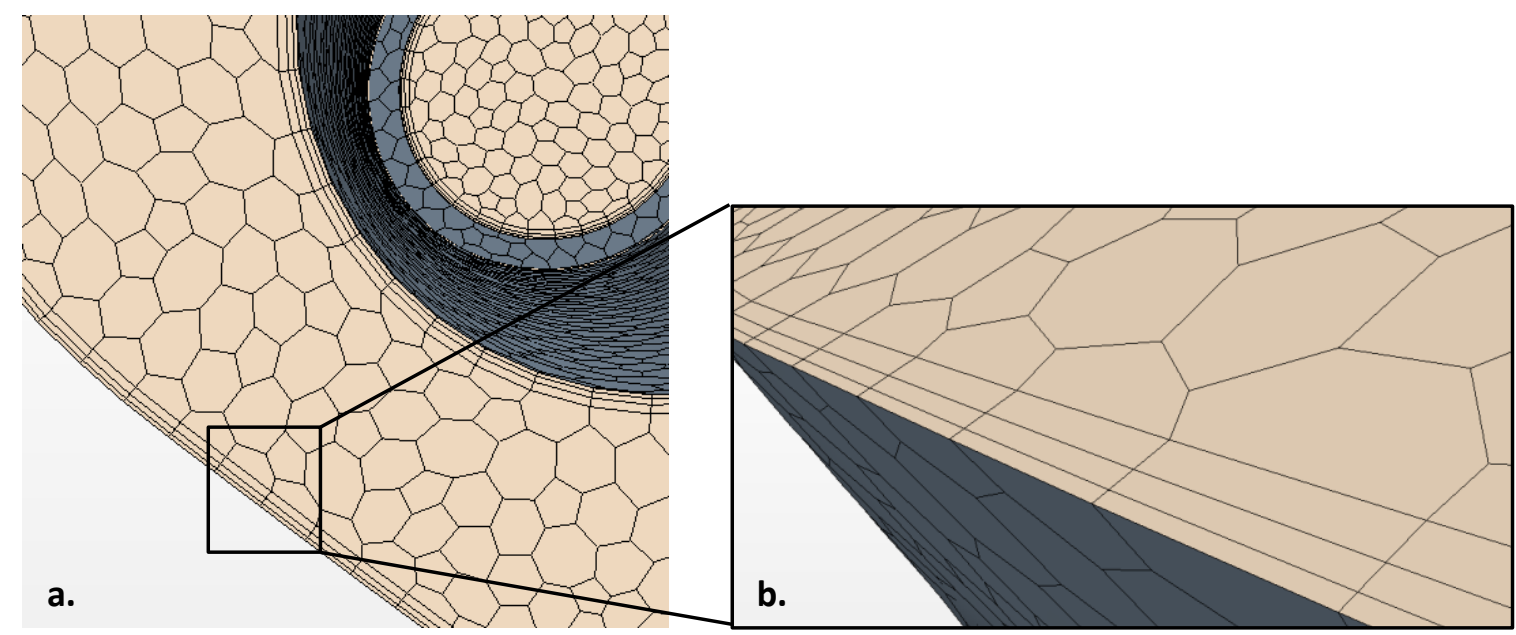

Figure 6.11. A close-up of the cells that encompass the polyhedral mesh (a) and a closeup of the 3 prism layers formed at each wall boundary (b). The prism layers are fixed at the fluid-artery wall interface so that no-slip wall boundary conditions could be accurately solved.

The prism layered mesh at the wall boundaries ensured that physics being solved near the walls were solved with sufficient accuracy. 
The mesh density and mesh size are discretization parameters that must be determined to yield accurate solutions in CFD modeling. The mesh quality in this study will be defined by the number of cells in the model. Table 6.7 lists the number of cells associated with three arbitrary heart models, as well as the time required to compute each mesh using two different computing hardware.

Table 6.7

Three LAD and LCX models chosen at random and their associated mesh quality and computational time using two discrete computing hardware.

\begin{tabular}{|c|c|c|c|c|} 
Human Heart Model & Base Size & $\begin{array}{c}\text { Mesh Quality } \\
\text { (Number of Cells) }\end{array}$ & \multicolumn{2}{c|}{$\begin{array}{c}\text { Mesh Conversion } \\
\text { Time }\end{array}$} \\
\hline $\begin{array}{l}\text { HH88 (LAD) } \\
\text { HH88 (LCX) }\end{array}$ & $0.00015 \mathrm{~m}$ & 799,705 & $2.86 \mathrm{~min}$ & WS2 \\
\hline $\begin{array}{l}\text { HH97 (LAD) } \\
\text { HH97 (LCX) }\end{array}$ & $0.00015 \mathrm{~m}$ & 599,609 & $3.37 \mathrm{~min}$ & --- \\
\hline HH178 (LAD) & $0.00015 \mathrm{~m}$ & 655,940 & $2.69 \mathrm{~min}$ & $7.34 \mathrm{~min}$ \\
\hline HH178 (LCX) & $0.00015 \mathrm{~m}$ & 535,340 & $3.14 \mathrm{~min}$ & --- \\
\hline
\end{tabular}

Note. WS1 and WS2 are workstations 1 and 2, respectively, comprising of different computer hardware. Details on the specifications of WS1 and WS2 computer hardware are listed in Chapter 3.

A mesh convergence study was executed for all artery models using a best practice convergence tolerance residual level of $1 \times 10^{-4}$ [106] for each of the steady state physics simulations. All LAD and LCX meshes contained approximately $8 \times 10^{5}$ cells which was sufficient for executing the CFD solvers in the blood domain.

The mesh for HH97 LAD was executed using WS1 and resulted in a volume meshing speed of 2.69 minutes (Table 6.7). In contrast, when the same operation was performed using WS2, the volume meshing speed was deferred to 7.34 minutes. WS1 was generally $2 \mathrm{x}$ faster at computing volume meshes compared to WS2, and was used as the primary machine to execute all computationally demanding mesh convergence 
analyses and STAR-CCM+ simulation runs. Table 6.8 illustrates the cell count for each of the simulated LAD and LCX models.

Table 6.8.

Cell count and corresponding work station for each LAD and LCX model executed.

\begin{tabular}{|c|c|c|c|c|}
\hline Model \# & Location & Base Size & Cell \# & WorkStation \\
\hline \multirow[t]{2}{*}{ HH55 } & LAD & \multirow{2}{*}{$0.00015 \mathrm{~m}$} & 823,707 & WS1 \\
\hline & $\mathrm{LCX}$ & & 940,164 & WS1 \\
\hline \multirow[t]{2}{*}{ HH88 } & LAD & \multirow{2}{*}{$0.00015 \mathrm{~m}$} & 799,705 & WS2 \\
\hline & LCX & & 599.609 & WS2 \\
\hline \multirow[t]{2}{*}{ HH97 } & LAD & \multirow{2}{*}{$0.00015 \mathrm{~m}$} & 655,940 & WS2 \\
\hline & $\mathrm{LCX}$ & & 535,340 & WS2 \\
\hline \multirow[t]{2}{*}{ HH102 } & LAD & \multirow{2}{*}{$0.00015 \mathrm{~m}$} & 343,759 & WS1 \\
\hline & LCX & & 911,245 & WS1 \\
\hline \multirow[t]{2}{*}{ HH178 } & LAD & \multirow{2}{*}{$0.00015 \mathrm{~m}$} & 812,517 & WS1 \\
\hline & LCX & & 677,797 & WS1 \\
\hline \multirow[t]{2}{*}{ HH259 } & LAD & \multirow{2}{*}{$0.00015 \mathrm{~m}$} & $1,534,272$ & WS2 \\
\hline & LCX & & $1,251,094$ & WS2 \\
\hline
\end{tabular}

Note. WorkStation represents the machine that was used to solve each HH simulation using STAR-CCM+.

The optimum base size of $0.00015 \mathrm{~m}$ used across all models was determined using a mesh convergence analysis executed for the HH178 LAD (Fig. 6.12) and HH88 LCX (Fig. 6.13). 

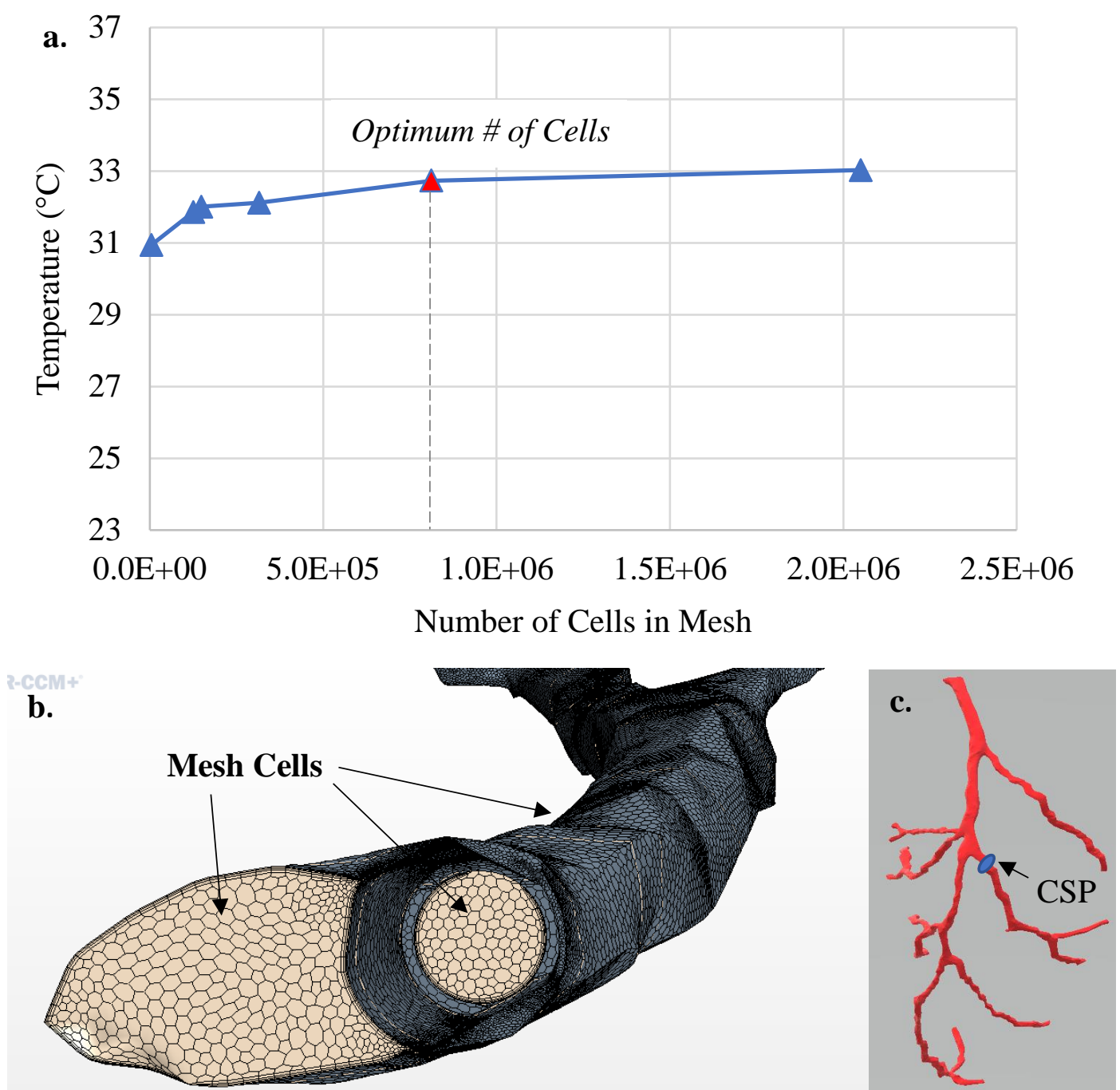

Figure 6.12. A total of six base sizes were chosen for each heart model and the resultant number of cells were plotted against the ABT (a) at a cross sectional plane (CSP) (c) in the artery network. A proximal mesh view (b) shows the cells lining the surface of the HH178 LAD model. A base size of $0.00015 \mathrm{~m}$ resulted in a reasonable number of cells $(\sim 8 \mathrm{e} 5)$ that led to invariant average bulk temperature at the CSP. 

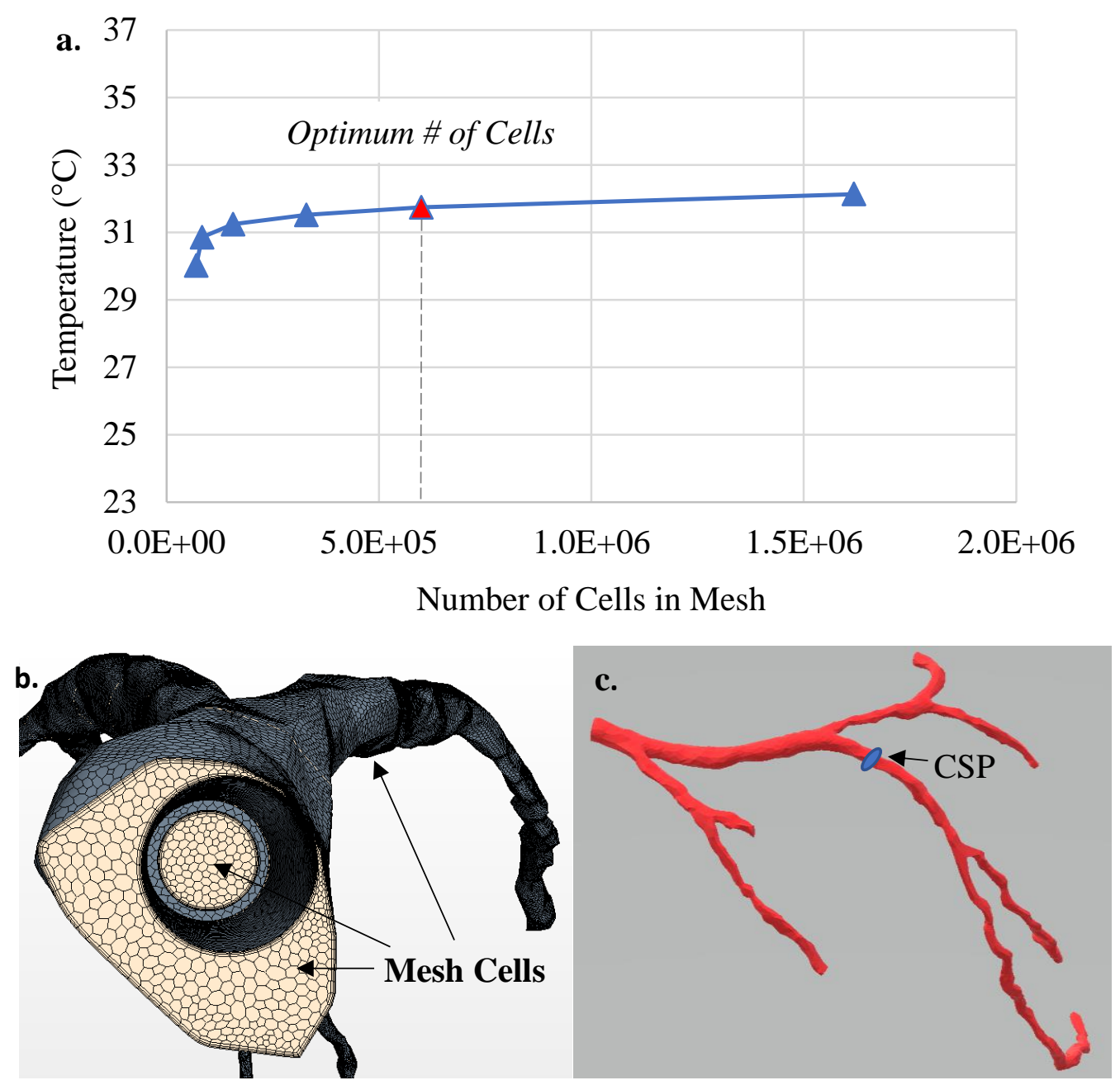

Figure 6.13. Six base sizes were chosen for heart HH88 LCX and the resultant number of cells were plotted against the ABT (a) at a cross sectional plane (CSP) (b). A proximal mesh view (b) shows the cells lining the surface of the HH88 LCX model. A base size of $0.00015 \mathrm{~m}$ also produced a mesh with ( 6e5) cells that yielded sufficient ABT results.

The red triangle in Figs. 6.12a and 6.13a present a reasonable base size (1.5e-4 m) to be used for determining accurate ABT's for all the models balancing solution accuracy vs computational cost. Optimal mesh size was chosen based on temperatures that were measurable and clinically relevant in the real world. In this case, thermocouples measuring GuideLiner® outlet temperatures (Table 4.6 in chapter 4) were calibrated to an accuracy of $\pm 0.36^{\circ} \mathrm{C}$. As a result, over half a million cells were reduced using a base size of $1.5 \mathrm{e}-4 \mathrm{~m}$ in most models, rather than $1.5 \mathrm{e}-4 \mathrm{~m}$, since the ABTs between the two mesh 
sizes varied within the thermocouples calibrated accuracy of $\pm 0.36^{\circ} \mathrm{C}$. The remaining $\mathrm{HH}$ mesh analysis studies were satisfactory since a tolerance of $0.5^{\circ} \mathrm{C}$ was deemed acceptable for this study and ABTs remained within $\pm 0.36^{\circ} \mathrm{C}$ at the GuideLiner® outlet.

\section{Thermal Fluid Model Testing Matrix}

A thermal fluid modeling matrix was created to explore all six patients. Each of the six LAD and LCX heart models required a total inlet flow rate of $75 \mathrm{~mL} / \mathrm{min}$ (Fig. 6.9) that was independent of GuideLiner ${ }^{\circledR}$ and blood flow rate (Table 6.3), which resulted in 36 total CFD models that needed to be organized and modeled correctly. Therefore, the thermal fluid model testing matrix (Table 6.9) was a critical component for the organization of this thesis work.

Table 6.9

Thermal fluid model testing matrix used to describe the catheter type, location, inlet conditions, and models used in this thesis work. Please see Appendix C for a more comprehensive version of the thermal fluid modeling matrix.

\begin{tabular}{|c|c|c|c|c|}
\hline \multicolumn{5}{|c|}{ 7F Cordis $₫$ Guide Catheter \& GuideLiner $₫$} \\
\hline \multicolumn{5}{|c|}{ HH55 } \\
\hline \multirow{2}{*}{$\begin{array}{c}\text { Location } \\
\text { (Proximal) }\end{array}$} & \multicolumn{3}{|c|}{ Flow Rate (ml/min) } & \multirow[t]{2}{*}{ Model \# } \\
\hline & Total & Blood & GuideLiner ${ }^{\circledR}$ & \\
\hline \multirow[t]{3}{*}{ LAD } & \multirow[t]{3}{*}{75} & 45.8 & 29.20 & HH55_1 \\
\hline & & 25.97 & 49.03 & HH55_2 \\
\hline & & 6.15 & 68.85 & HH55_3 \\
\hline \multirow[t]{3}{*}{ LCX } & \multirow[t]{3}{*}{75} & 45.8 & 29.20 & HH55_4 \\
\hline & & 25.97 & 49.03 & HH55_5 \\
\hline & & 6.15 & 68.85 & HH55_6 \\
\hline \multicolumn{5}{|c|}{ HH88 } \\
\hline \multirow{2}{*}{$\begin{array}{c}\text { Location } \\
\text { (Proximal) }\end{array}$} & \multicolumn{3}{|c|}{ Flow Rate (ml/min) } & \multirow[t]{2}{*}{ Model \# } \\
\hline & Total & Blood & GuideLiner ${ }^{\circledR}$ & \\
\hline \multirow[t]{3}{*}{ LAD } & \multirow[t]{3}{*}{75} & 45.8 & 29.20 & HH88_1 \\
\hline & & 25.97 & 49.03 & HH88_2 \\
\hline & & 6.15 & 68.85 & HH88_3 \\
\hline \multirow[t]{3}{*}{ LCX } & \multirow[t]{3}{*}{75} & 45.8 & 29.20 & HH88_4 \\
\hline & & 25.97 & 49.03 & HH88_5 \\
\hline & & 6.15 & 68.85 & HH88_6 \\
\hline
\end{tabular}


Table 6.9

Thermal fluid model testing matrix continued.

\begin{tabular}{|c|c|c|c|c|}
\hline \multicolumn{5}{|c|}{ HH97 } \\
\hline \multirow{2}{*}{$\begin{array}{c}\text { Location } \\
\text { (Proximal) }\end{array}$} & \multicolumn{3}{|c|}{ Flow Rate (ml/min) } & \multirow[t]{2}{*}{ Model \# } \\
\hline & Total & Blood & GuideLiner ${ }^{\circledR}$ & \\
\hline \multirow[t]{3}{*}{ LAD } & \multirow[t]{3}{*}{75} & 45.8 & 29.20 & HH97_1 \\
\hline & & 25.97 & 49.03 & HH97_2 \\
\hline & & 6.15 & 68.85 & HH97_3 \\
\hline \multirow[t]{3}{*}{ LCX } & \multirow[t]{3}{*}{75} & 45.8 & 29.20 & HH97_4 \\
\hline & & 25.97 & 49.03 & HH97_5 \\
\hline & & 6.15 & 68.85 & HH97_6 \\
\hline \multicolumn{5}{|c|}{ HH102 } \\
\hline \multirow{2}{*}{$\begin{array}{c}\text { Location } \\
\text { (Proximal) }\end{array}$} & \multicolumn{3}{|c|}{ Flow Rate (ml/min) } & \multirow[t]{2}{*}{ Model \# } \\
\hline & Total & Blood & GuideLiner ${ }^{\circledR}$ & \\
\hline \multirow[t]{3}{*}{ LAD } & \multirow[t]{3}{*}{75} & 45.8 & 29.20 & HH102_1 \\
\hline & & 25.97 & 49.03 & HH102_2 \\
\hline & & 6.15 & 68.85 & HH102_3 \\
\hline \multirow[t]{3}{*}{ LCX } & \multirow[t]{3}{*}{75} & 45.8 & 29.20 & HH102_4 \\
\hline & & 25.97 & 49.03 & HH102_5 \\
\hline & & 6.15 & 68.85 & HH102_6 \\
\hline \multicolumn{5}{|c|}{ HH178 } \\
\hline \multirow{2}{*}{$\begin{array}{c}\text { Location } \\
\text { (Proximal) }\end{array}$} & \multicolumn{3}{|c|}{ Flow Rate (ml/min) } & \multirow[t]{2}{*}{ Model \# } \\
\hline & Total & Blood & GuideLiner ${ }^{\circledR}$ & \\
\hline \multirow[t]{3}{*}{ LAD } & \multirow[t]{3}{*}{75} & 45.8 & 29.20 & HH178_1 \\
\hline & & 25.97 & 49.03 & HH178_2 \\
\hline & & 6.15 & 68.85 & HH178_3 \\
\hline \multirow[t]{3}{*}{ LCX } & \multirow[t]{3}{*}{75} & 45.8 & 29.20 & HH178_4 \\
\hline & & 25.97 & 49.03 & HH178_5 \\
\hline & & 6.15 & 68.85 & HH178_6 \\
\hline \multicolumn{5}{|c|}{ HH259 } \\
\hline \multirow{2}{*}{$\begin{array}{c}\text { Location } \\
\text { (Proximal) }\end{array}$} & \multicolumn{3}{|c|}{ Flow Rate (ml/min) } & \multirow[t]{2}{*}{ Model \# } \\
\hline & Total & Blood & GuideLiner ${ }^{\circledR}$ & \\
\hline \multirow[t]{3}{*}{ LAD } & 75 & 45.8 & 29.20 & HH259_1 \\
\hline & & 25.97 & 49.03 & HH259_2 \\
\hline & & 6.15 & 68.85 & HH259_3 \\
\hline LCX & 75 & 45.8 & 29.20 & HH259_4 \\
\hline & & 25.97 & 49.03 & HH259_5 \\
\hline & & 6.15 & 68.85 & HH259_6 \\
\hline
\end{tabular}

Each of the heart models in the thermal-fluid model testing matrix (Table 6.9) were executed in STAR-CCM+ using the methods described in this chapter. The results 
of each CFD simulation were used to predict the impact of each patient-specific heart structure on cooling effectiveness. A rationalization for how cooling effectiveness was defined, as well as a parametric study descibing the impact of patient-specific heart structure is descibed in detail in Chapter 7. 


\section{Chapter 7}

\section{Thermal Fluid Model Predictions}

\section{Overview}

Studies involving the biophysics of heart cooling remain largely unexplored, specifically, the biophysics of coronary artery cooling following myocardial infarction. Experimental studies have explored myocardial protection techniques using computer simulations to examine topical cardiac cooling [101], and conjugate heat transfer by pumping cold liquid through the major cardiac chambers [102].

Computational vascular research has focused primarily on computing and visualizing hemodynamics (i.e. coronary blood shear, pressure, and velocity) [4][103][104][105], coronary artery fractional flow reserve (FFR) [106], and lumped parameter modeling [75][107]. A lumped parameter model is a mathematical model that describes the physiological constraints of a system using parameters that are less complex, hence lumped [108]. To our understanding, there is no published literature analyzing the biophysics of localized cooling in patient-specific coronary arteries.

In this study, patient specific left anterior descending (LAD) and left circumflex (LCX) artery models are used to explore the impact of vascular geometry on thermal fluid behavior in the coronary arteries [13]. A new thermal model is introduced that could provide a better understanding of the biophysics of acute myocardial infarction (AMI) during $\mathrm{TH}$, as well as provide insight to guide more efficient $\mathrm{TH}$ device designs and operation parameters [92].

The objective of this study is to create thermal fluid models of six patient-specific left main coronary arteries (LMCAs). The goal is to predict arterial blood temperatures for a variety of hearts and catheter infusion flow rates in the proximal LAD and LCX vasculature. The following computational fluid dynamics (CFD) models can help 
determine and qualitatively analyze temperature distributions in the LMCA's and can help shed light on TH device design and operation to optimize patient outcomes following AMI.

\section{Parametric Study - Sensitivity Analysis}

Our parametric study will explore three questions:

1) What is the impact of heart structure on cooling effectiveness?

2) What is the impact of TH device location placement on cooling effectiveness?

3) Is there a surface area to volume $(\mathrm{SA}: \mathrm{V})$ ratio that reveals cooling effectiveness?

Before these questions can be answered, a basic knowledge of heat transport in physiological systems is required.

Heat transport in physiological systems is a very complicated phenomenon [109]. The heart circulates blood flow through vascular systems that reduce in size with each branching network until the blood reaches the capillaries. From the capillaries, small intravenous vessels are filled with blood which eventually flows into larger vessels that bring blood back into the hearts circulation system [109]. Figure 7.1 shows the general impact of blood flow in successively smaller vessels in tissue [149]. 


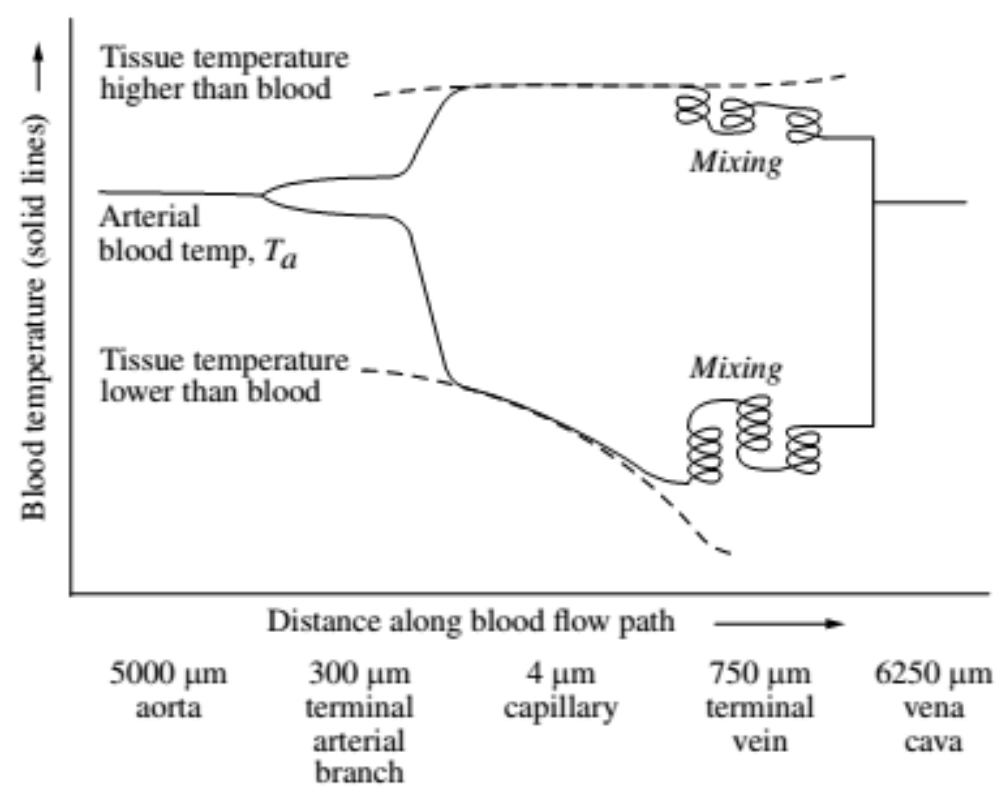

Figure 7.1. The blood temperature as it navigates through blood vessels of varying diameters, as proposed by Chen and Homes [149]. The dashed line indicates the range of the tissue temperature surrounding the vessels. Most temperature changes occur in smaller vessels, whereas large vessels retain blood flow path temperatures for longer distances.

As seen in Fig. 7.1, most of the temperature change occurs in the smaller vessels, which contributes to the fact that blood flow temperatures drastically differ from surrounding tissue when moderately large vessels experience blood flow. Thus, thermal equilibrium between blood vessels and the surrounding tissue is greater in vessels with smaller diameters. However, since the scope of this work is limited to the impact of cooling in the blood flow paths of coronary artery networks, optimizing the depth of cooled blood flow path temperatures in patient-specific models will be the focus of this work.

ABT defined. To accurately measure blood flow in blood vessels and artery networks, an average bulk mass flow rate can be calculated to determine the mean flow rate at any particular cross-section in the vessel of interest. Therefore, to accurately 
measure a vessel's mean temperature distribution at any given cross-section, the average bulk temperature (ABT) can be determined.

The ABT of a fluids cross-section is calculated in STAR-CCM+ using the following equation (Eq. 2.9) from Chapter 2 [115]:

$$
T_{A B T}=\frac{2}{u_{m} r_{o}^{2}} \int_{0}^{r_{o}} u T(r, x) r d r
$$

where $T_{A B T}$ is the average bulk temperature of the fluid, $u_{m}$ is the mean velocity, and $r_{o}$ is the radius of the cross section of interest.

ABT in patient-specific vascular structures. To determine the ABT for each patient-specific heart model, cross-sectional planes were created in STAR-CCM+ that started at the artery inlet and extended down each LAD and LCX mother vessel in $5 \mathrm{~mm}$ increments until the planes reached the outlet. The significance of the mother vessel is that it comprises of the main fluid domain that carries blood from the LMCA ostia to each of the distal LAD and LCX coronary artery trees. A visual representation of the crosssectional planes created in the LAD (Fig. 7.2) and LCX (Fig. 7.3) arteries shows the mother vessel used in all human heart $(\mathrm{HH})$ models. 


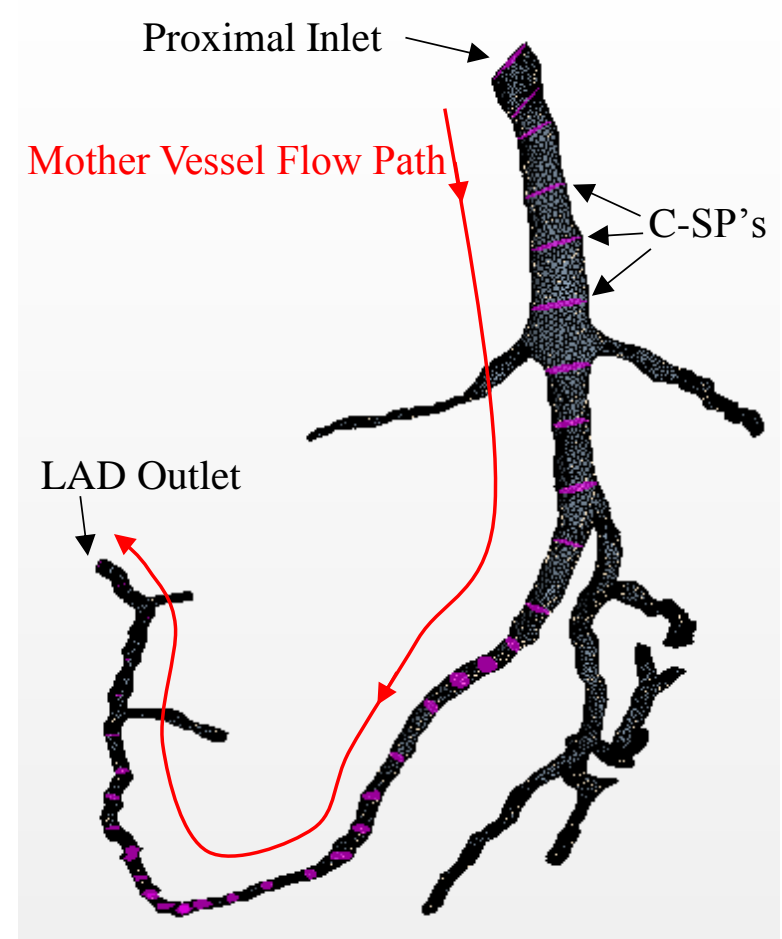

Figure 7.2. Cross-sectional planes (C-SP's) spaced 5mm apart extending from HH259 LAD inlet to the outlet. The mother vessel for each LAD human heart $(\mathrm{HH})$ model originated at the proximal inlet and extended to each distal LAD outlet.

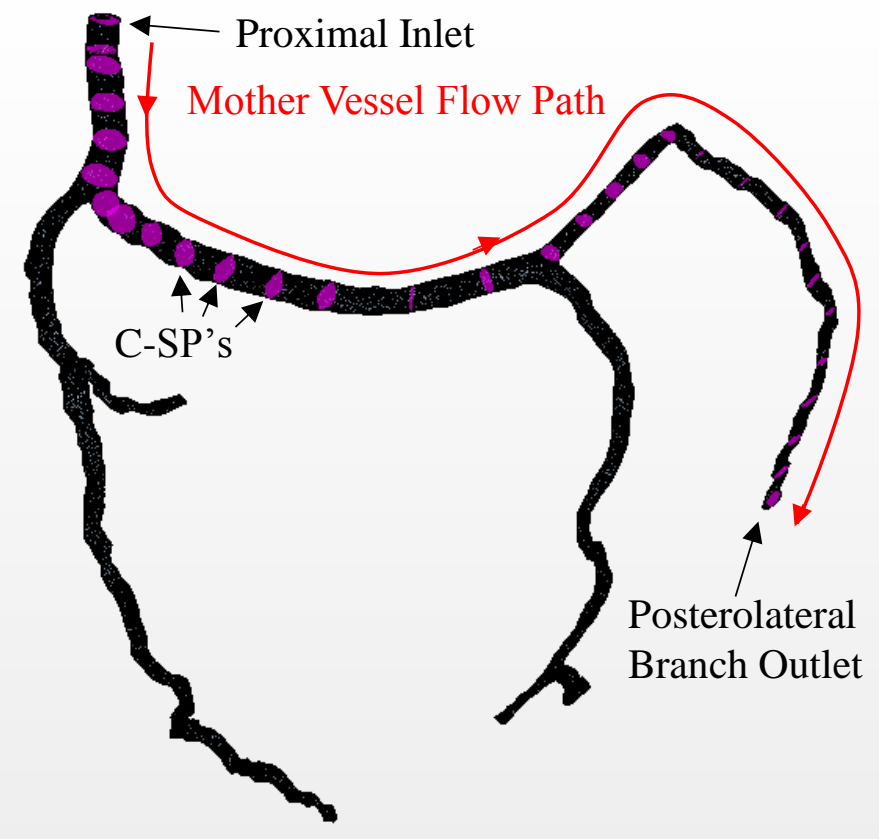

Figure 7.3. Cross-sectional planes (C-SP's) spaced 5mm apart extending from $\mathrm{HH} 259$ LCX inlet to the outlet. The mother vessel for each LCX human heart (HH) model originated at the proximal inlet and extended to each distal posterolateral branch outlet. 
To determine the impact of coronary vascular structure on cooling effectiveness (CE), a basic ratio was created with the intent of being simple and analogous to fractional flow reserve (FFR). FFR is a simple calculation used during intracoronary procedures to determine the pressure difference across an occlusion [105]. Similar to FFR, CE was determined as the ratio of the length of the therapeutic hypothermia average bulk temperature (TH-ABT) in the mother vessel over the entire length of the mother vessel (Eq. 7.1).

$$
\text { Cooling Effectiveness }(C E)=\frac{\text { Distance from Inlet where } A B T<35^{\circ} \mathrm{C}(\mathrm{mm})}{\text { Total Length of Mother Vessel }(\mathrm{mm})}
$$

We chose $\mathrm{ABTs}<35^{\circ} \mathrm{C}$ for our studies based on Dae et al. 2018, which conducted a study analyzing six randomized endovascular cooling trails involving 629 patients [78]. As a result, temperatures $<35^{\circ} \mathrm{C}$ reduced infarct size from $6.5 \%$ in control studies to $30 \%$. Therefore, ABTs $<35^{\circ} \mathrm{C}$ were defined as the cut-off temperature for CE. A visual representation of how $\mathrm{CE}$ was applied to each mother vessel flow path is described in Figure 7.4. 
$50 \%$ CE

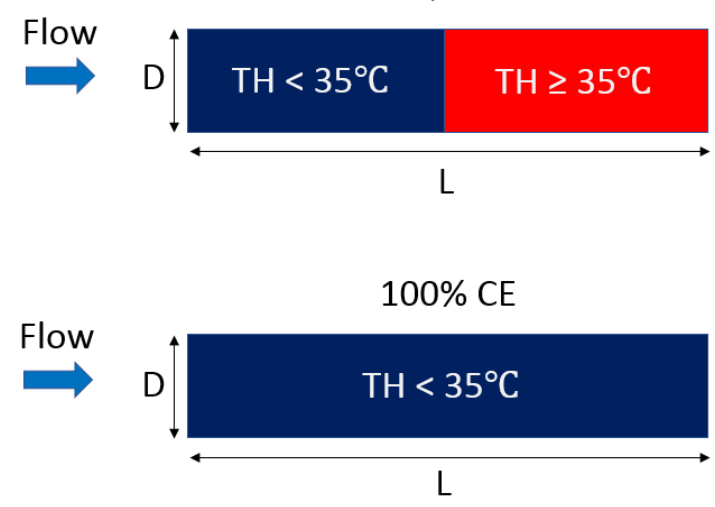

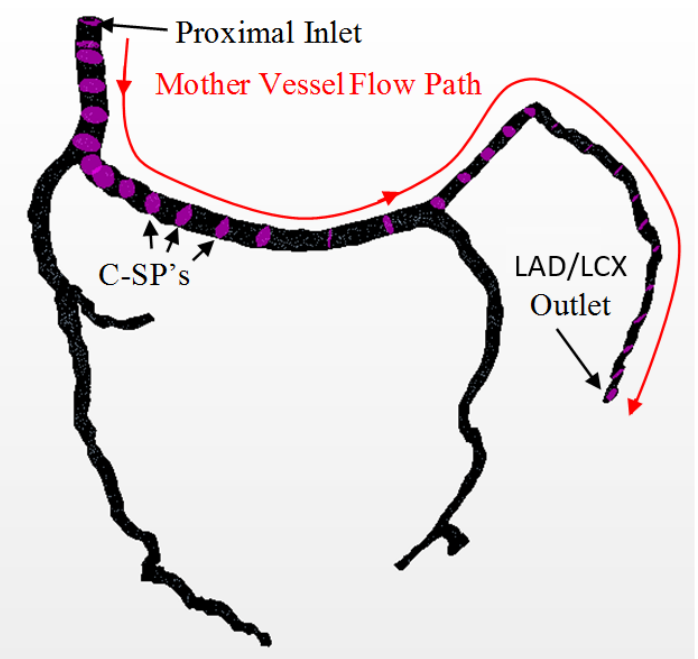

Figure 7.4. Cooling effectiveness (CE) represents the ratio of the distance from the proximal inlet where TH-ABTs are evident in the mother vessel over the entire length of the mother vessel. If TH-ABTs are evident up to and including half the length of the vessel, then this vessel would have 50\% CE. Furthermore, if TH-ABTs were measured at the outlet, then the vessel would have $100 \%$ CE.

The cooling effectiveness calculation (Eq. 7.1) was used as a post-processing method and was applied to all CFD simulations comprising of six HH models and 29.20, 49.03, $68.85 \mathrm{~mL} / \mathrm{min}$ GuideLiner® flow rates.

ABT in single heart with single flow rate. After the cross-sectional planes were created in the LAD and LCX arteries for each patient-specific HH model, the thermal fluid simulations were executed in STAR-CCM+ using a single flow rate obtained from the in vitro mock loop study for each $\mathrm{HH}$ patient model. The ABT for each cross-sectional plane along the artery's fluid flow path were plotted for the LAD (Fig. 7.5) and LCX (Fig. 7.6) from $\mathrm{HH} 178$. 

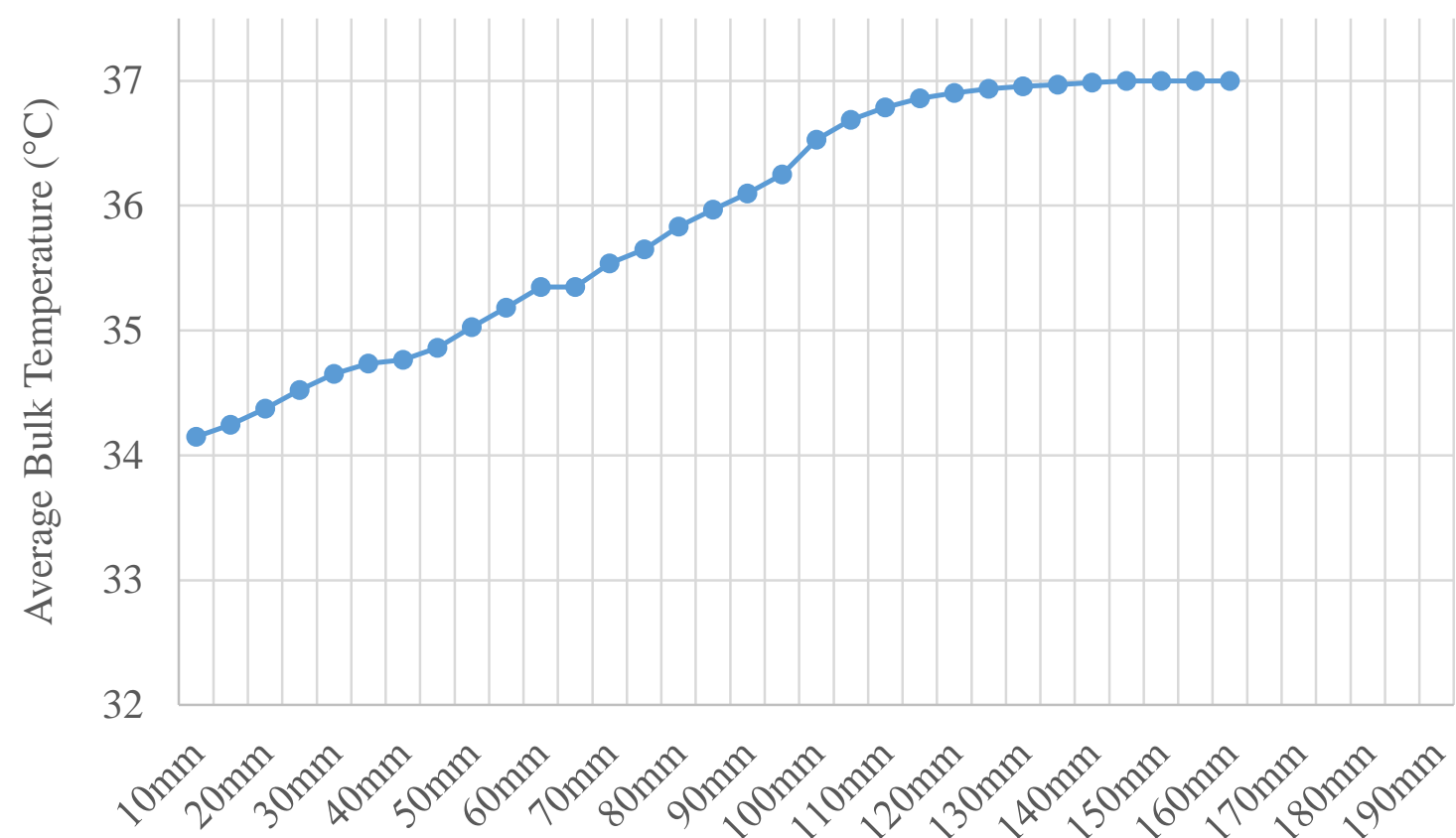

Distance From HH178 LAD Inlet

Figure 7.5. ABT along the LAD mother vessel flow path with single GuideLiner® flow rate at $29.2 \mathrm{~mL} / \mathrm{min}$ for $\mathrm{HH} 178$.

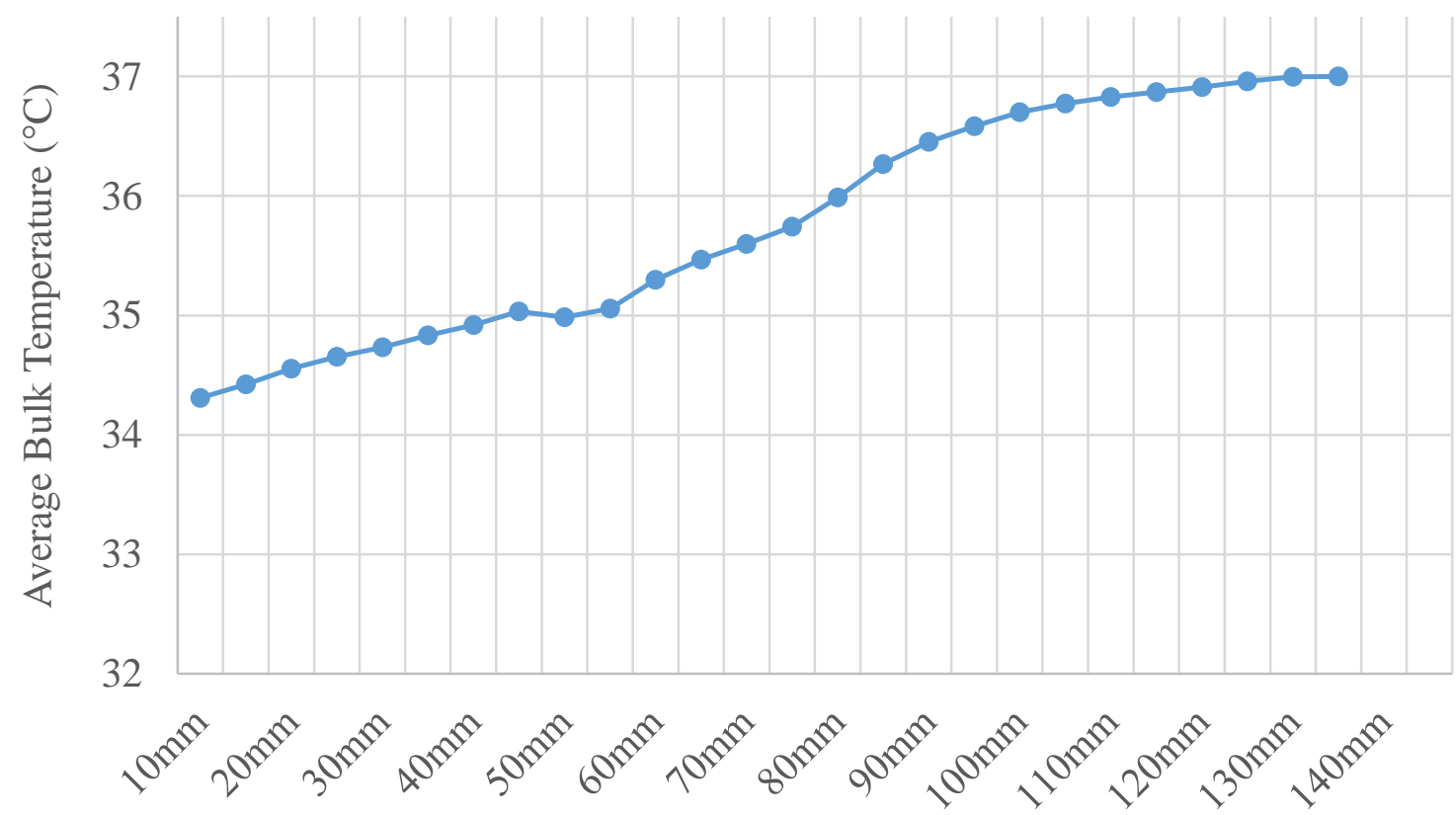

Distance From HH178 LCX Inlet

Figure 7.6. ABT along the LCX mother vessel flow path with single GuideLiner ${ }^{\circledR}$ flow rate at $29.2 \mathrm{~mL} / \mathrm{min}$ for $\mathrm{HH} 178$. 
As seen in the above figures, a proximal GuideLiner® flow rate of $29.2 \mathrm{~mL} / \mathrm{min}$ in each LAD and LCX for patient HH178 resulted in dissimilar ABTs trends extending along the mother vessel. The $\mathrm{LAD}$ resulted in an $\mathrm{ABT}$ of $34.86^{\circ} \mathrm{C}$ reaching $45 \mathrm{~mm}$ into the artery, while the LCX resulted in $34.92^{\circ} \mathrm{C} \mathrm{ABT}$ reaching $40 \mathrm{~mm}$ in the artery. This indicates that the anterior descending and left circumflex artery mother vessel flow paths within patients have variability in cooling potential outcomes.

ABT in all hearts with single flow rate. Cross-sectional planes were created along the flow path in $5 \mathrm{~mm}$ increments for each of the six representative heart $(\mathrm{RH})$ models. All LAD (Fig. 7.7) and LCX (Fig. 7.8) RH models were executed in STAR-CCM+ using a $29.4^{\circ} \mathrm{C}$ infusate fluid mixture (as described in Chapter 6) at a flow rate of $29.2 \mathrm{ml} / \mathrm{min}$, which is similar to a $30 \mathrm{ml} / \mathrm{min}$ flow rate used in transcatheter studies to deliver saline in the proximal left main coronary arteries (LMCA) [99]. 


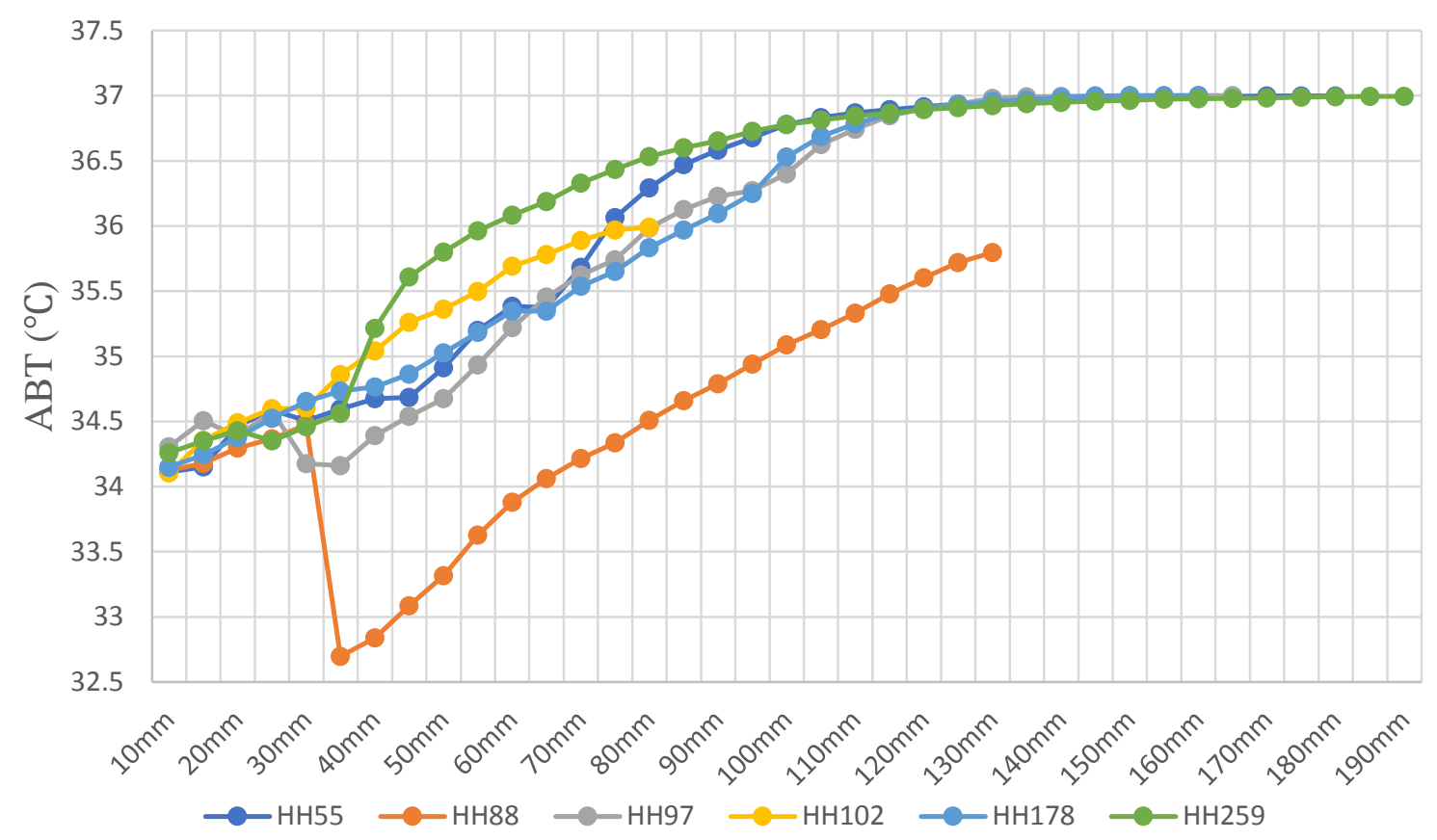

Figure 7.7. All LAD representative heart models using a $29.4^{\circ} \mathrm{C}$ infusate fluid mixture at a flowrate of $29.2 \mathrm{ml} / \mathrm{min}$. Temperature drop in HH88 and HH97, at locations of about 30$40 \mathrm{~mm}$, are discussed in detail in the next section.

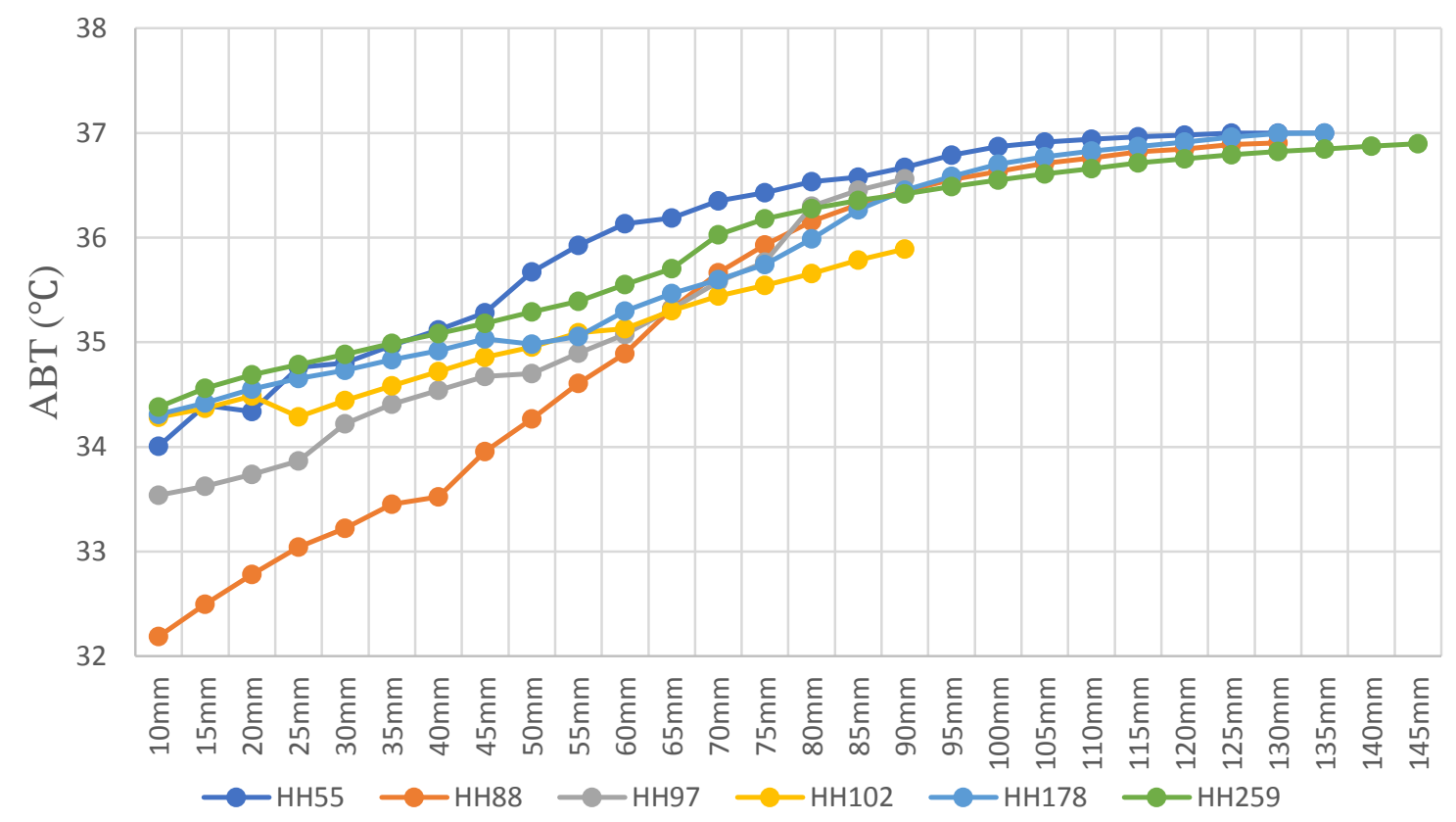

Figure 7.8. All LCX representative heart models using a $29.4^{\circ} \mathrm{C}$ infusate fluid mixture at a flowrate of $29.2 \mathrm{ml} / \mathrm{min}$. 
Since each representative heart $(\mathrm{RH})$ model was infused with an identical GuideLiner® flow rate $(29.2 \mathrm{~mL} / \mathrm{min})$ and temperature $\left(29.4^{\circ} \mathrm{C}\right) 3-5 \mathrm{~mm}$ into the proximal artery, the results were localized to the $10 \mathrm{~mm}$ plane (Figs. 7.7 and 7.8) for consistency. Figure 7.7 describes the impact of biovariability in the LAD using a single GuideLiner® flow rate, which resulted in $\mathrm{HH} 88$ experiencing good cooling potential where $\mathrm{ABTs}<35^{\circ} \mathrm{C}$ reached $100 \mathrm{~mm}$ into the mother vessel, as compared to $\mathrm{HH} 259$ where $\mathrm{ABTs}<35^{\circ} \mathrm{Creached}$ only 40mm into the mother vessel. Similarly, HH88 in the LCX experienced the greatest cooling potential while HH55 experienced the poorest, reaching warmer temperatures quicker.

ABT in single heart with multiple flow rates. The thermal fluid simulation for patient HH259 was executed using the three catheter flow rates obtained from the in vitro mock loop study; $29.2 \mathrm{ml} / \mathrm{min}, 49.03 \mathrm{ml} / \mathrm{min}$, and $68.85 \mathrm{ml} / \mathrm{min}$. The ABT for each crosssectional plane along the artery's fluid flow path was plotted for each LAD (Fig. 7.9) and LCX (Fig. 7.10) artery. 


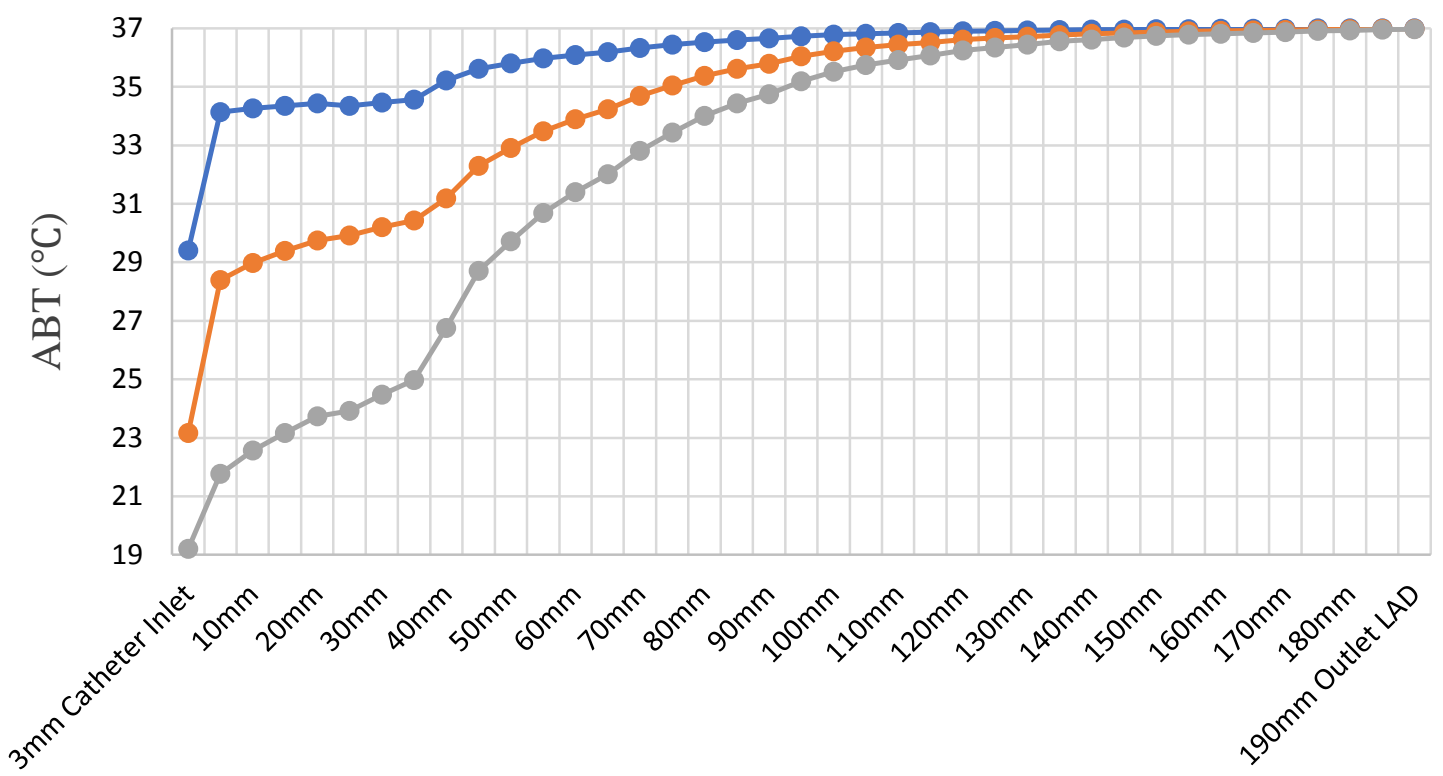

Distance From HH259 LAD Inlet

$\longrightarrow 29.2 \mathrm{ml} / \mathrm{min} \longrightarrow 49.03 \mathrm{ml} / \mathrm{min} \longrightarrow 68.85 \mathrm{ml} / \mathrm{min}$

Figure 7.9. Results of ABT at each cross-sectional plane location for HH259 LAD using flow rates obtained from in vitro mock loop study; $29.2 \mathrm{ml} / \mathrm{min}, 49.03 \mathrm{ml} / \mathrm{min}$, and 68.85 $\mathrm{ml} / \mathrm{min}$. The rapid spike in $\mathrm{ABT}$ at the $40 \mathrm{~mm}$ plane is the result of a bifurcated septal perforator branch that deviated from the mother vessel. 


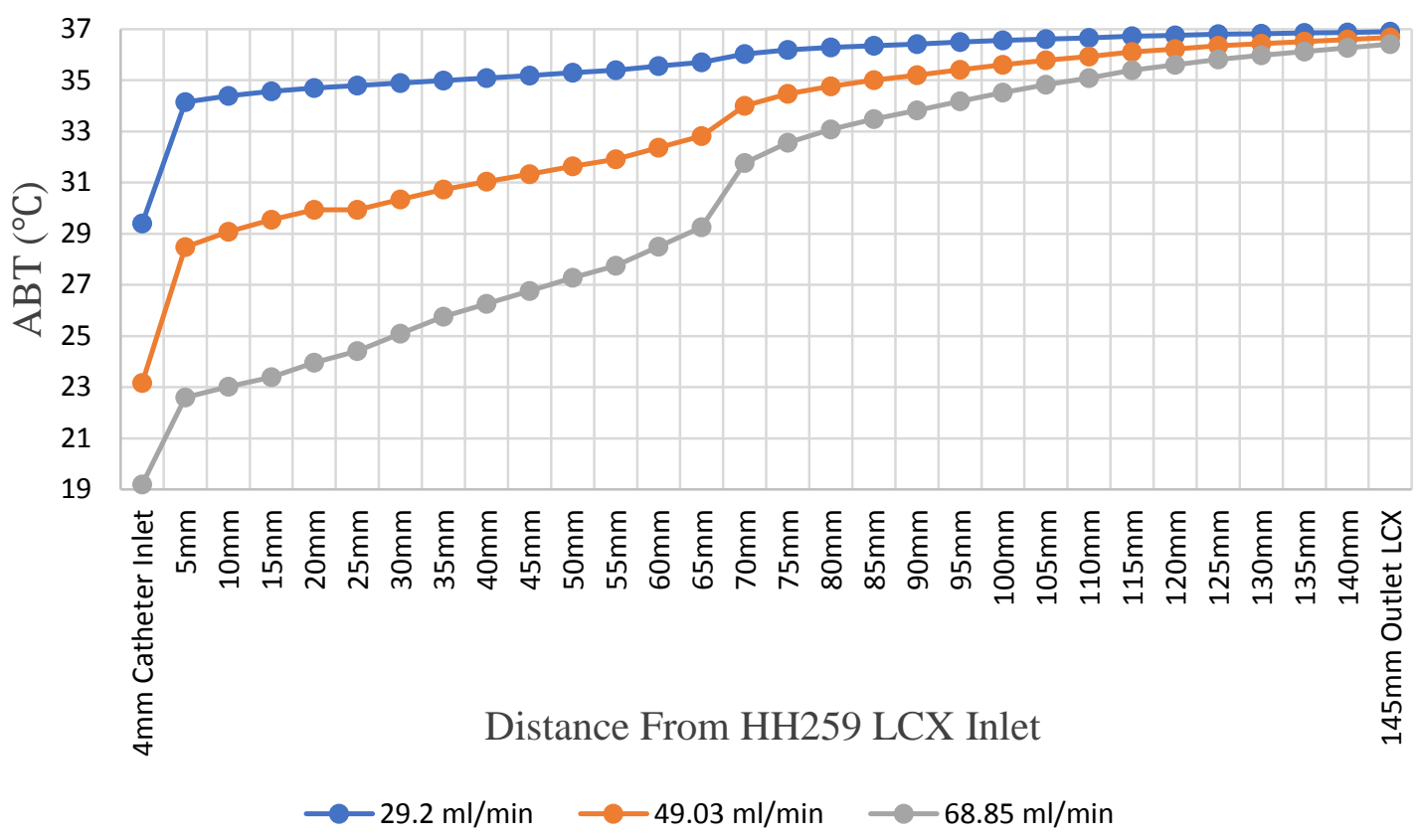

Figure 7.10. Results of ABT vs cross-sectional plane location for HH259 LCX using flow rates obtained from in vitro mock loop study; $29.2 \mathrm{ml} / \mathrm{min}, 49.03 \mathrm{ml} / \mathrm{min}$, and 68.85 $\mathrm{ml} / \mathrm{min}$. The rapid spike in ABT at the $70 \mathrm{~mm}$ plane resulted in a bifurcation from the second obtuse marginal branch that deviated from the mother vessel.

As predicted by Chen and Homes [149], most of the temperature change occurred in smaller vessels downstream between $3000 \mu \mathrm{m}$ and $1000 \mu \mathrm{m}$, which suggests that thermal equilibrium between the vessel walls and the temperature of the fluid is significantly greater in vessels with smaller diameters. Our results indicate that abrupt ABT drops at or around $1000 \mu \mathrm{m}$ diameters generally occur between $40-55 \mathrm{~mm}$ from the catheter inlet, which falls within the arterial diameters of Chen and Homes findings [142]. Additionally, the $\mathrm{ABT}$ in the $\mathrm{LAD}$ reached $36.55^{\circ} \mathrm{C}$ at $135 \mathrm{~mm}$ from the artery inlet (Fig. 7.9) using 68.85 $\mathrm{ml} / \mathrm{min}$ infusate flow. For the $\mathrm{LCX}$, the ABT resulted in $36.12^{\circ} \mathrm{C}$ at $135 \mathrm{~mm}$ from the inlet (Fig. 7.10) using $68.85 \mathrm{ml} / \mathrm{min}$ infusate flow.

Although both ABTs from Figure 7.9 and 7.10 were recorded at $135 \mathrm{~mm}$ downstream from the artery inlet, the cross-sectional area for the LAD plane (Fig. 7.11) 
was smaller than the cross-sectional area of the LCX plane (Fig. 7.12), which justifies the increased $\mathrm{ABT}$ in the LAD plane.

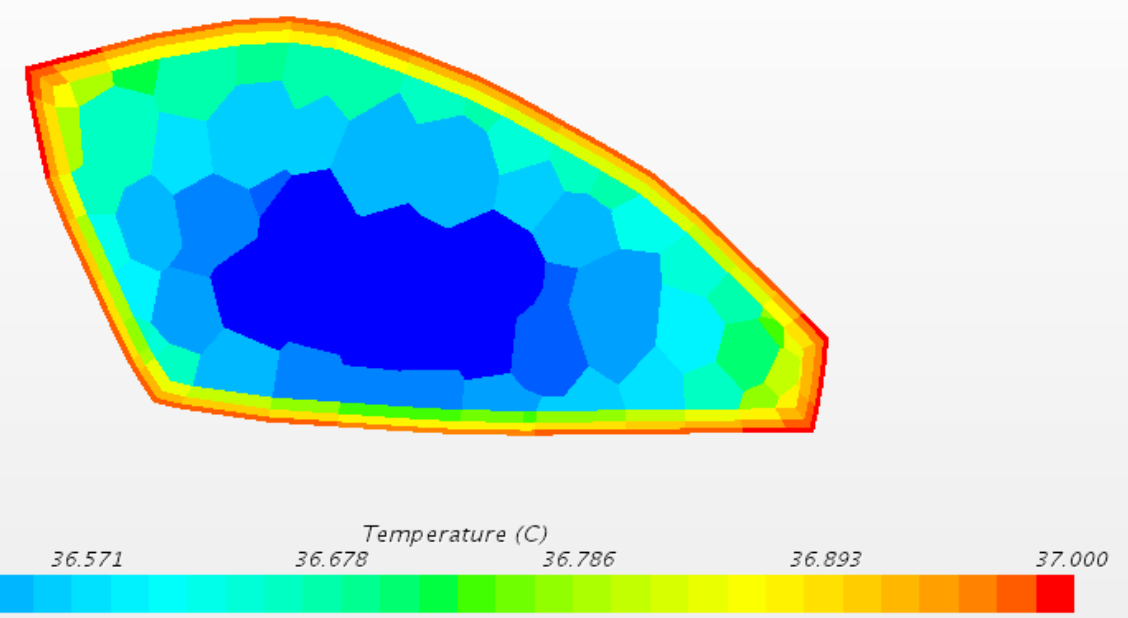

Figure 7.11. Cross-sectional area of the $135 \mathrm{~mm}$ cross-sectional plane from HH259 LAD. Note: Cross-sectional area: $1.935 \mathrm{e}-2 \mathrm{~mm}^{2}$. Average bulk temperature (ABT): $36.55^{\circ} \mathrm{C}$ at $68.85 \mathrm{ml} / \mathrm{min}$ infusate flow.

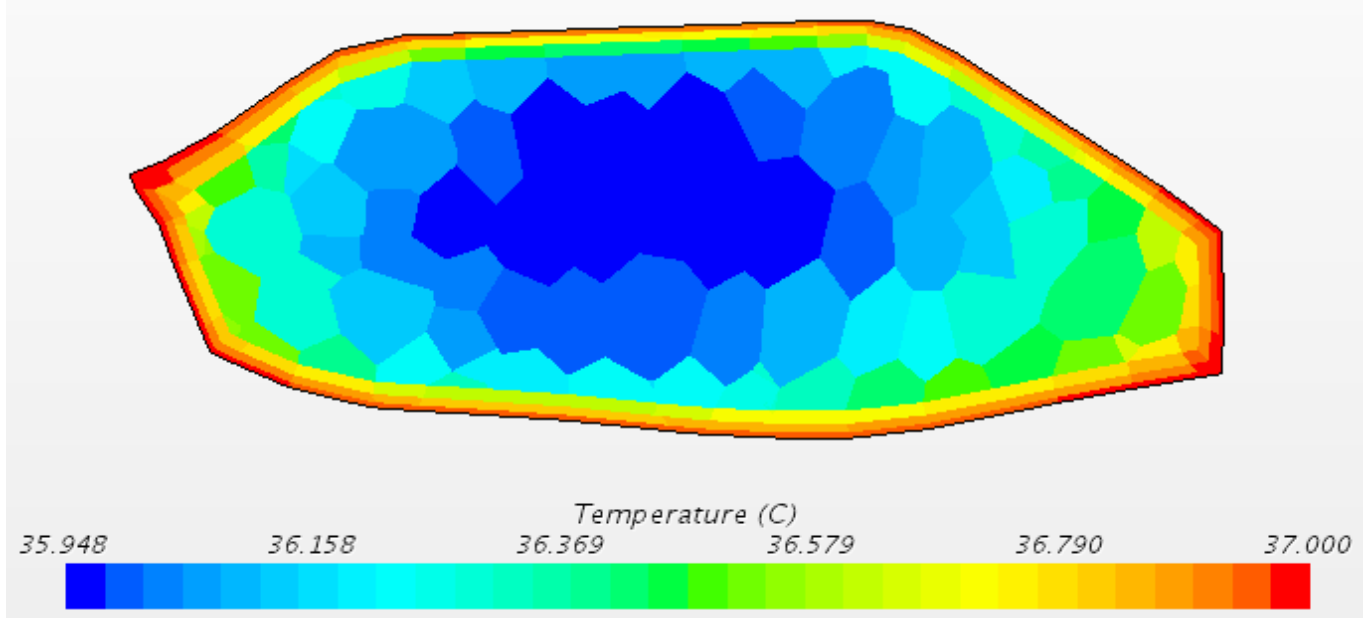

Figure 7.12. Cross-sectional area of the $135 \mathrm{~mm}$ cross-sectional plane from HH259 LCX. Note: Cross-sectional area: $1.968 \mathrm{e}-2 \mathrm{~mm}^{2}$. Average bulk temperature (ABT): $36.12^{\circ} \mathrm{C}$ at $68.85 \mathrm{ml} / \mathrm{min}$ infusate flow. 
Cooler temperatures set the foundation for greater cooling rates. The colder the GuideLiner® flow is, the more quickly TH-ABTs can reach into the distal arteries. As evident in Figures 7.9 and 7.10, GuideLiner® flow rates of $68.85 \mathrm{~mL} / \mathrm{min}$ most quickly cooled and reached the greatest cooling potential along the length of the artery. Furthermore, these models do not predict tissue cooling rates, but rather, ABT distributions of the coronary artery fluid flow path in each patient-specific $\mathrm{HH}$ model.

ABT in all hearts with multiple flow rates. The concluding ABT study involved analyzing the impact of biovariability on cooling effectiveness across all six patientspecific heart models using all three GuideLiner® flow rates.

In the event of an AMI, clinicians considering localized cooling treatment methods should consider the impact of biovariability from patient to patient and the probable outcomes that could result using variable GuideLiner® flow rates. To help describe this phenomenon, we analyzed the ABTs along each HH model's mother vessel flow path for all hearts using all GuideLiner® flow rates. Final results for the LAD and LCX mother vessel flow paths are prominent in Figure 7.13 and Figure 7.14, respectively. 

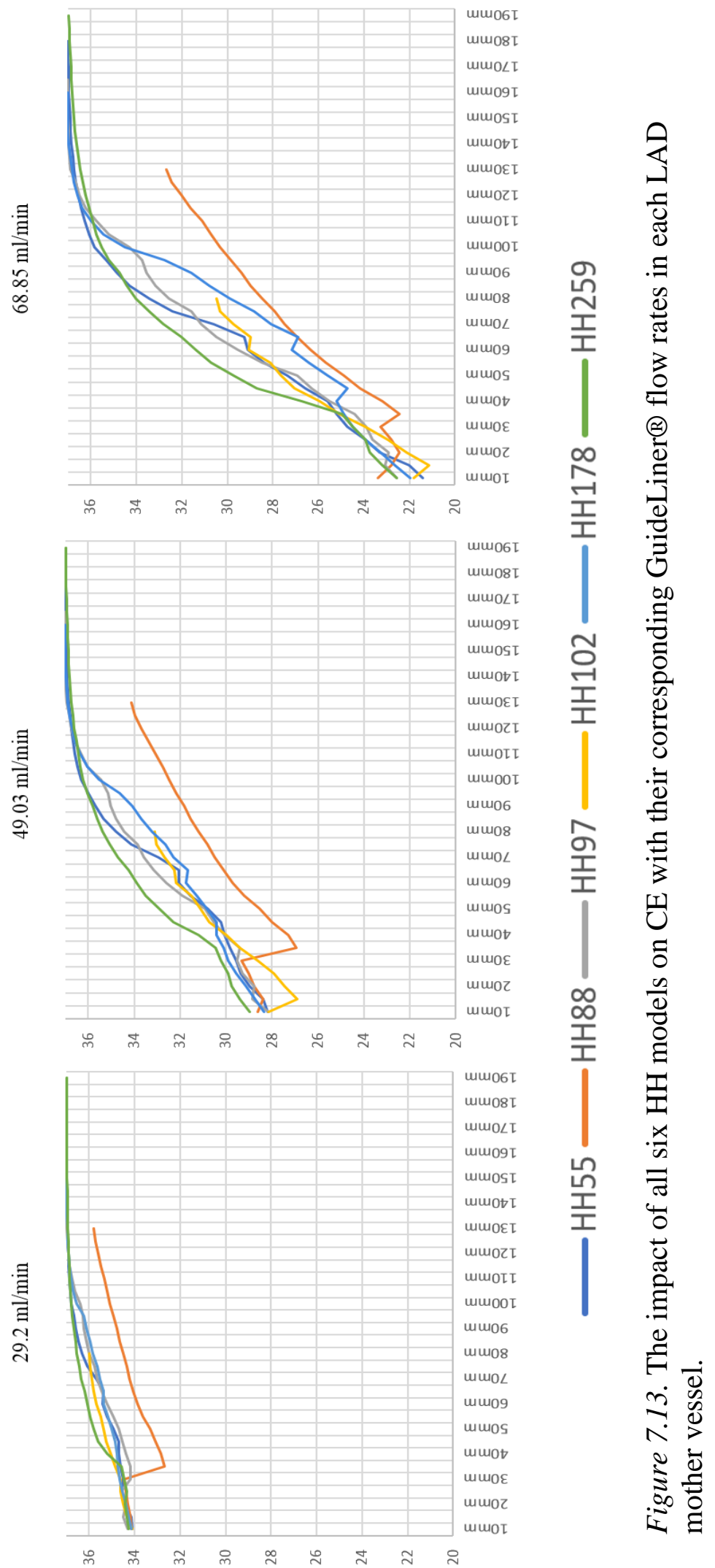

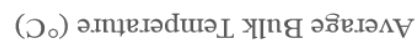



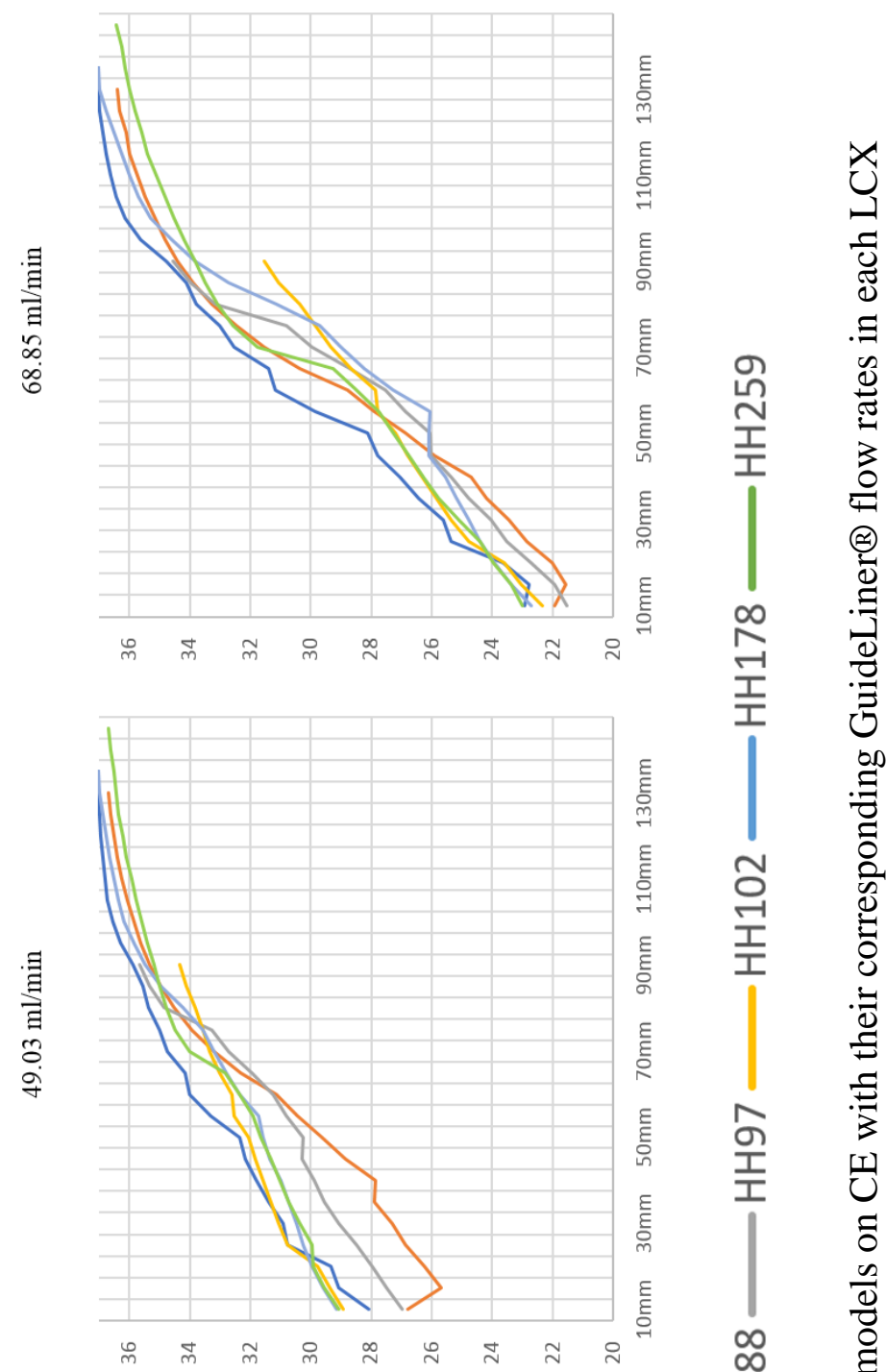

会告

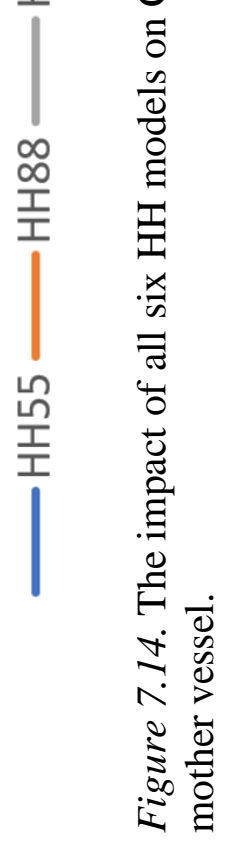

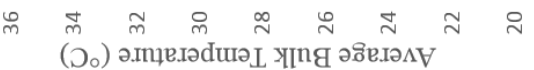


The summary of findings herein describe the impact of biovariability between patient-specific LMCA vascular structures on proximal GuideLiner® flow rates. Higher GuideLiner ${ }^{\circledR}$ flow rates resulted in deeper ABT cooling potential in both the LAD and LCX arteries. Although CE varied between patients, GuideLiner® flow rate was the dominant factor for reaching TH-ABTs in the distal coronary arteries. Further analysis of these findings are described in greater detail later in this chapter.

ABT dip in HH88 and HH97. A temperature drop was evident in heart HH88 and was suspected to be an artifact issue in the STAR-CCM+ physics solvers. The drop occurred between the 30 and $35 \mathrm{~mm}$ planes and dropped in $\mathrm{ABT}$ from $34.47^{\circ} \mathrm{C}$ to $32.7^{\circ} \mathrm{C}$. This physical phenomenon could suggest that there was a mysterious thermal energy sink, where thermal energy was removed in the blood stream downstream to produce lower temperatures in the artery network, which is not physically accurate. According to the first law of thermodynamics, energy can neither be created nor destroyed in an isolated system [150]. Therefore, further investigation was made to analyze our method for obtaining ABT. Both LAD ABT distributions for patient HH88 (Fig. 7.15) and HH97 (Fig. 7.16) consisted of having noticeable artifact issues and were further analyzed. 


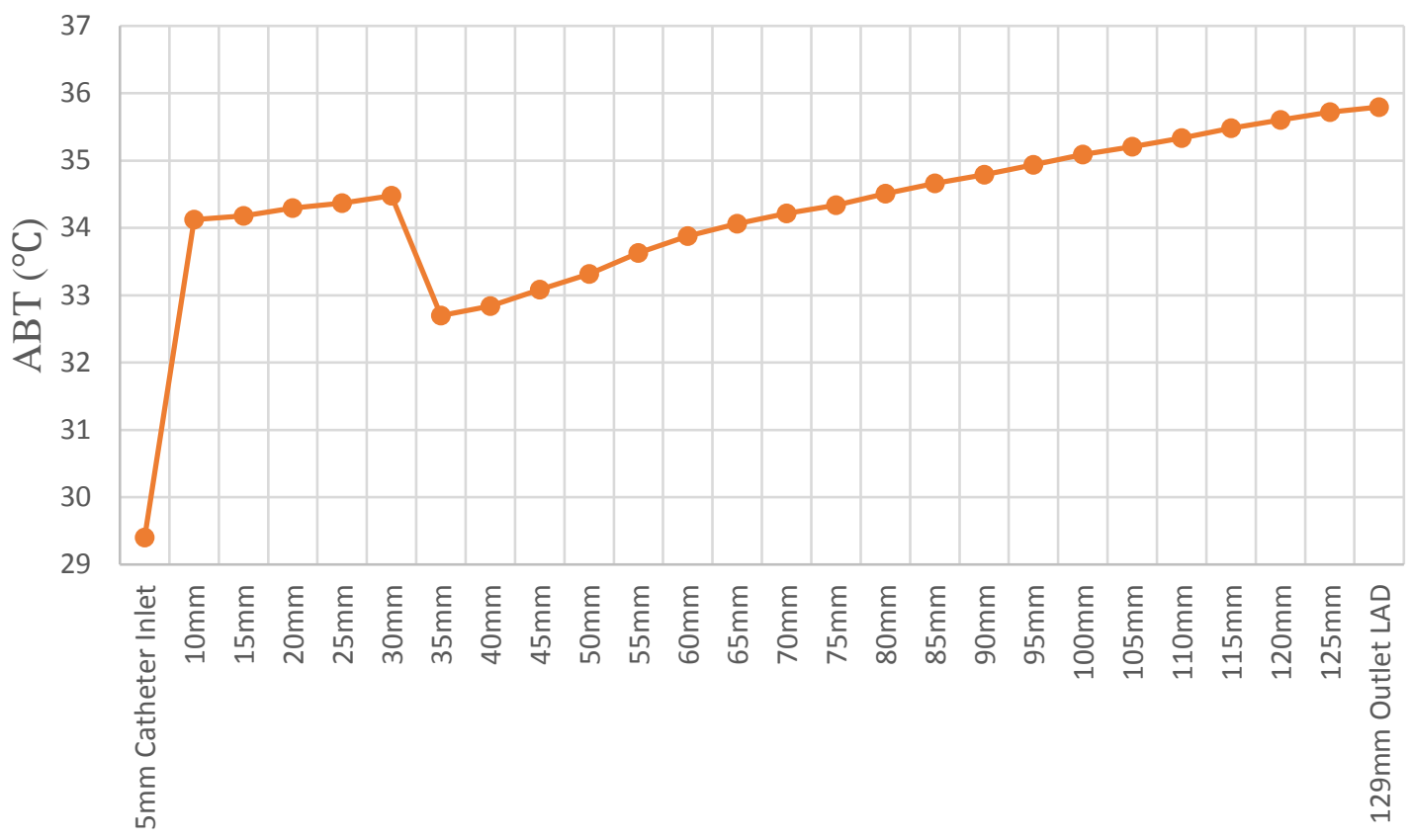

Distance From HH88 LAD Inlet

Figure 7.15. ABT distribution along mother vessel of patient HH88 LAD with artifact issue occurring between planes $30 \mathrm{~mm}$ and $35 \mathrm{~mm}$, at $29.2 \mathrm{ml} / \mathrm{min}$. The ABT steadily increases until the thermal fluid flow path exits the LAD outlet.

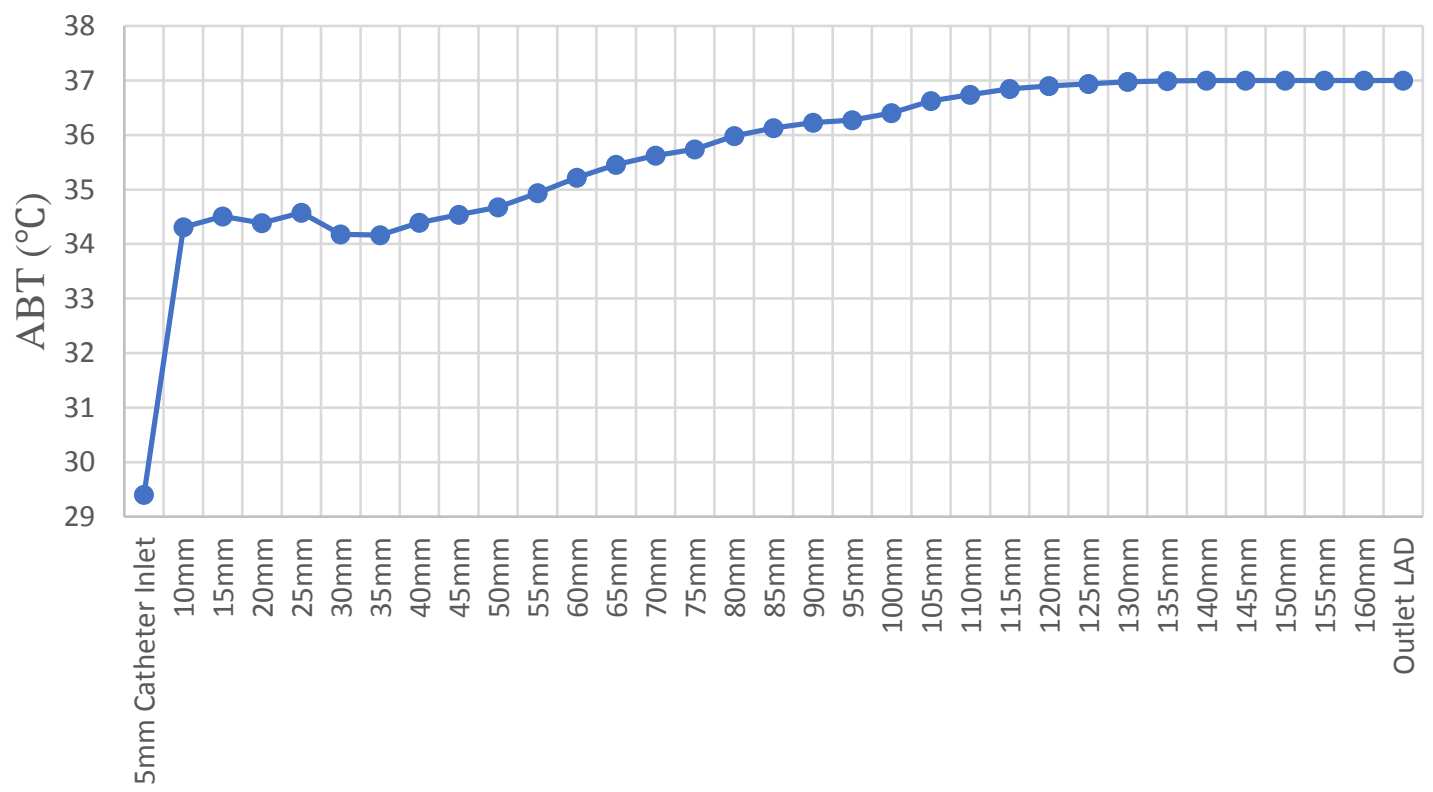

Distance From HH97 LAD Inlet

Figure 7.16. ABT distribution along flow path of patient HH97 LAD with numerous artifact issues originating at the $20 \mathrm{~mm}$ plane. GuideLiner ${ }^{\circledR}$ infusate flow rate: $29.2 \mathrm{ml} / \mathrm{min}$. 
After much consideration, it was determined that the assumed artifact in Figures 7.15 and 7.16 were not associated with the inherent approximations of the finite volume method, but rather, an issue concerning the stratification of flow. To dive deep in to this issue of stratification, rather than creating cross-sectional planes only along the mother vessel, we created a continuation of planes in $5 \mathrm{~mm}$ increments along each bifurcated branch (Fig. 7.17) that had deviated from the mother vessel.

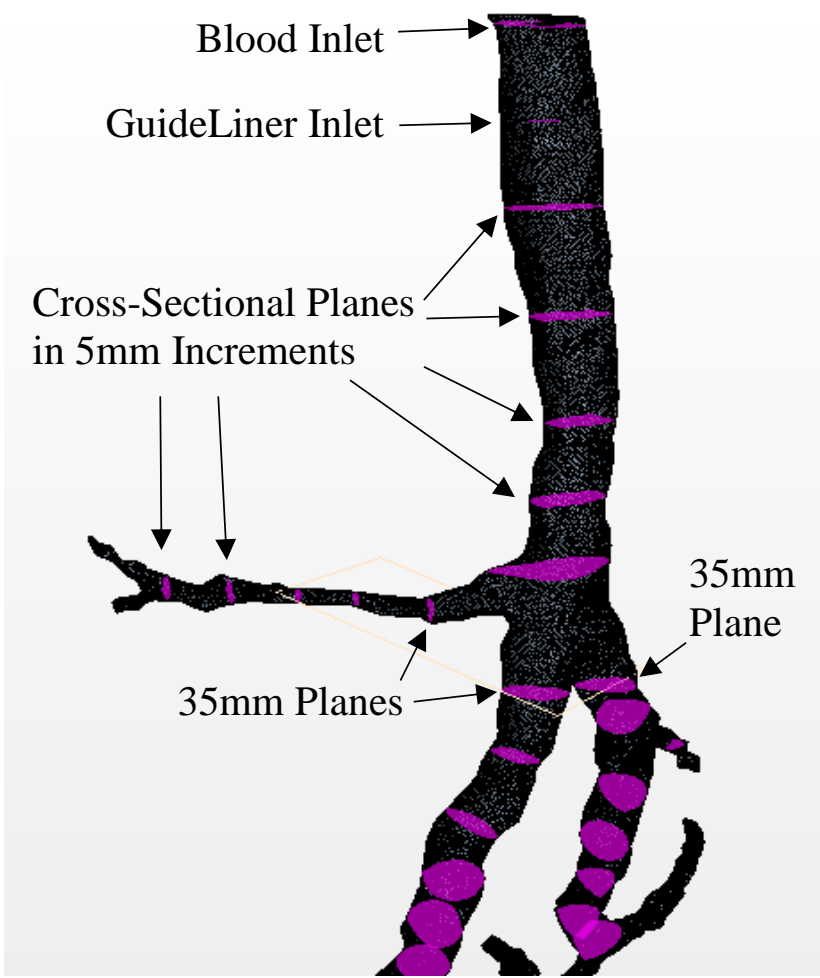

Figure 7.17. Continuation of planes in $5 \mathrm{~mm}$ increments along each bifurcated branch that had deviated from the mother vessel in HH88 LAD.

ABT cross-sectional planes extended from the proximal LAD inlet and spread into each bifurcated artery in $5 \mathrm{~mm}$ increments. Each individual ABT plane that extended down the artery network was grouped with the ABT plane that extended an equal distance from the proximal inlet. For example, all three $35 \mathrm{~mm}$ planes (Fig. 7.17) were summed together 
to determine a final ABT that resembled a stratified fluid mixture $35 \mathrm{~mm}$ from the artery inlet. The summation of planes was determined using the following equation:

$$
\text { ABT Summation }=\left(\frac{1}{\dot{m}_{1}+\dot{m}_{2}+\cdots}\right)\left(\left(\dot{m}_{1} A B T_{1}\right)+\left(\dot{m}_{2} A B T_{2}\right)+\cdots\right)
$$

where $\dot{m}_{1}$ and $\dot{m}_{2}$ are the flowrates of branches 1 and 2, respectively, and $A B T_{1}$ and $A B T_{2}$ are the average bulk temperatures of branches 1 and 2, respectively.

An image of the temperature distribution for the $35 \mathrm{~mm}$ planes from HH88 LAD (Fig. 7.18) helps to visualize the stratification of flow.
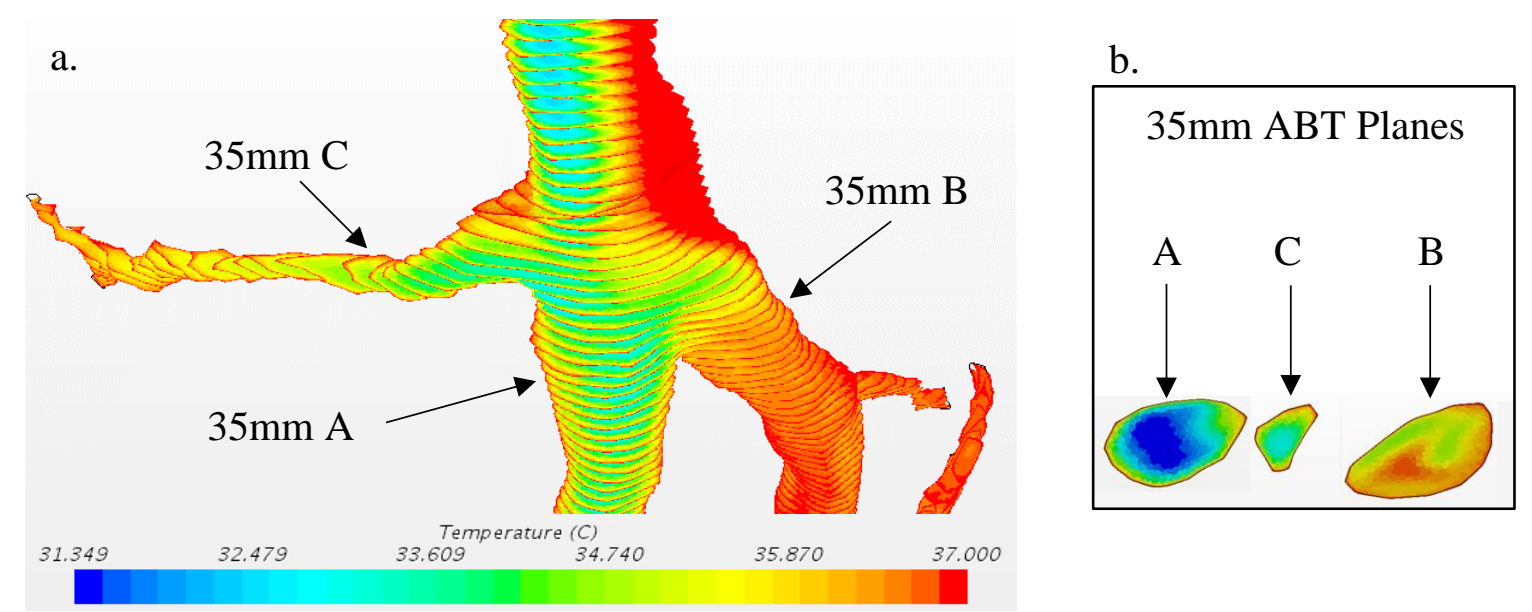

Figure 7.18. HH88 LAD bifurcations of $35 \mathrm{~mm}$ branches A, B, and C (a). The majority of chilled infusate flowed into branch A and only a minute amount passed into branches $\mathrm{B}$ and $\mathrm{C}$. The cross-sectional $35 \mathrm{~mm}$ ABT planes (b) are summed together in STAR-CCM+ using a form of Eq. 7.1 to produce combined ABT values.

The ABT's for branches A, B, and C from HH88 LAD were plotted (Fig. 7.19) to visualize the flow in each bifurcation between the $10 \mathrm{~mm}$ and $65 \mathrm{~mm}$ planes (see Appendix D for a qualitative analysis of how ABT contours change along the fluid flow path in a simple $3 \mathrm{D}$ tube). 


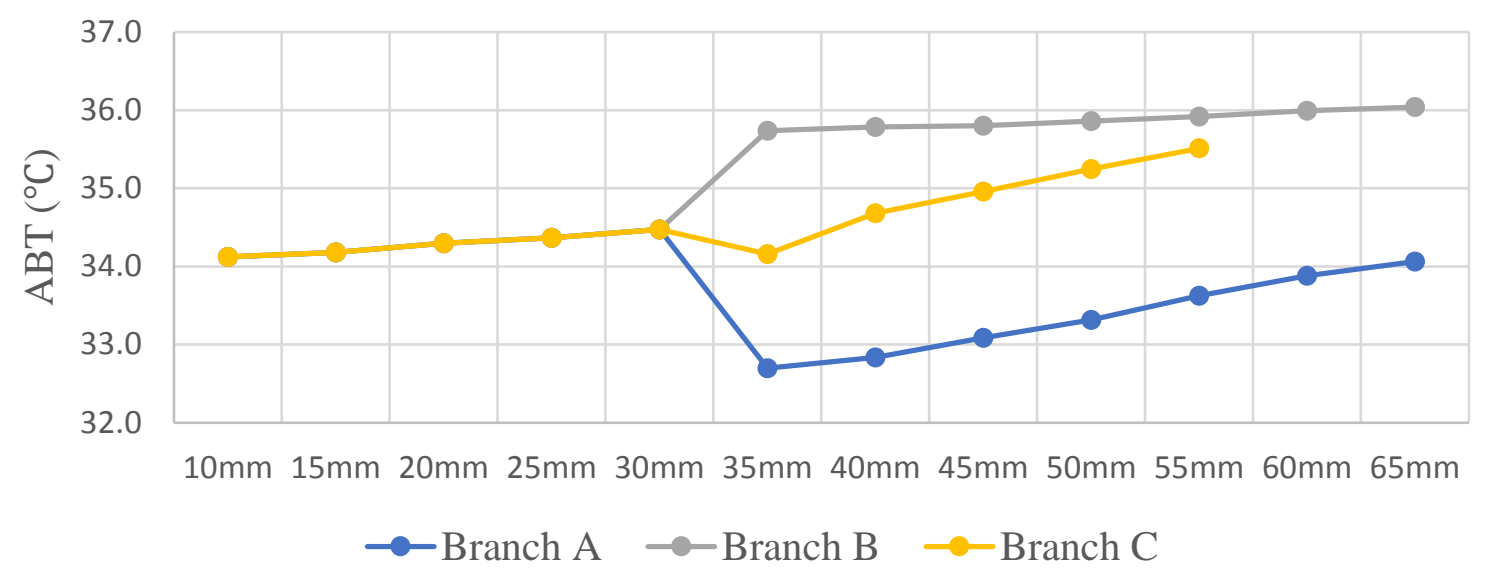

Figure 7.19. HH88 LAD with $29.2 \mathrm{ml} / \mathrm{min}$ proximal infusate flow comparing the ABT planes for branches A, B, and C.

STAR-CCM+ post-processing allowed us to calculate the ABT summations (Eq. 7.1) for each location along the artery pathway by creating an average bulk temperature report. The results of each stratified ABT were plotted as a function of distance along the artery (Fig. 7.20), which resulted in a plot with no identifiable artifact or stratification of flow.

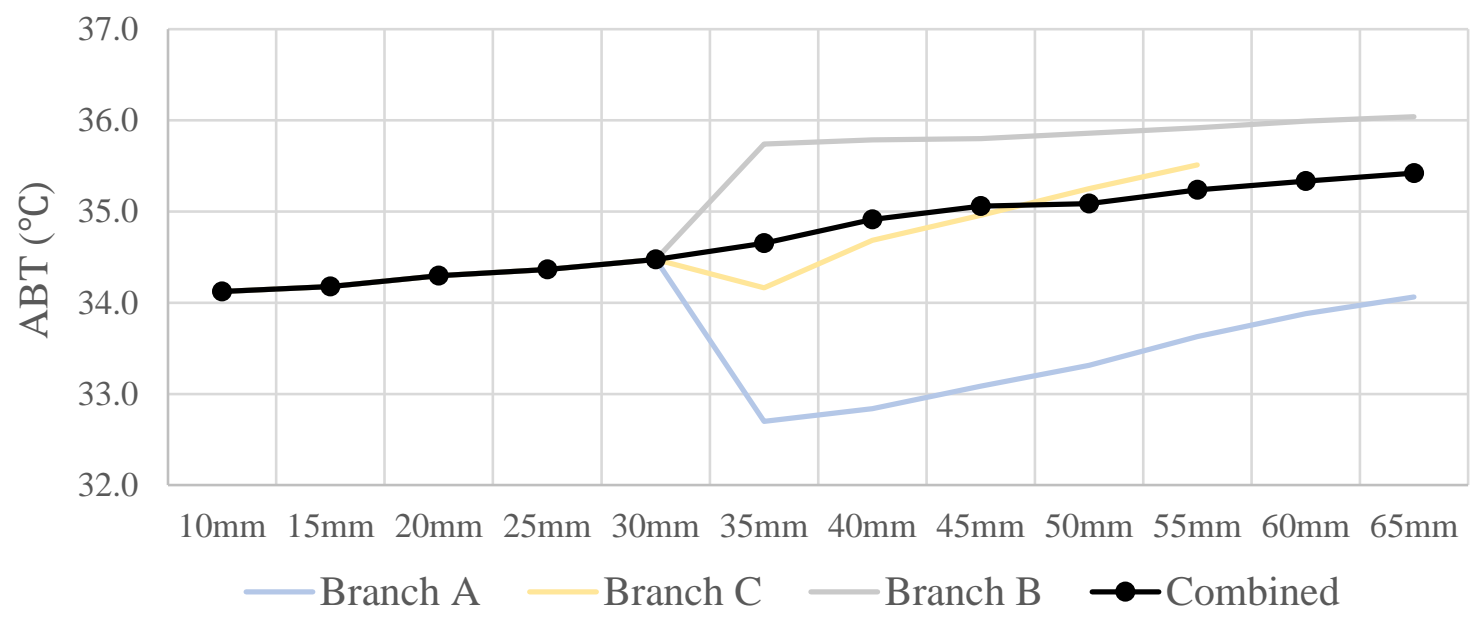

Figure 7.20. GuideLiner@ flow rate $(29.2 \mathrm{~mL} / \mathrm{min})$ in HH88 LAD with no impact of flow stratification using ABT summation from branches A, B, and C. The ABT's for each corresponding distance were summed together to determine a final ABT at each distance extending down the artery network. 
The flow stratification from HH97 was resolved using the same method from HH88. Cross-sectional planes were created in $5 \mathrm{~mm}$ increments that extended down the mother vessel (Fig. 7.21) of each bifurcation.

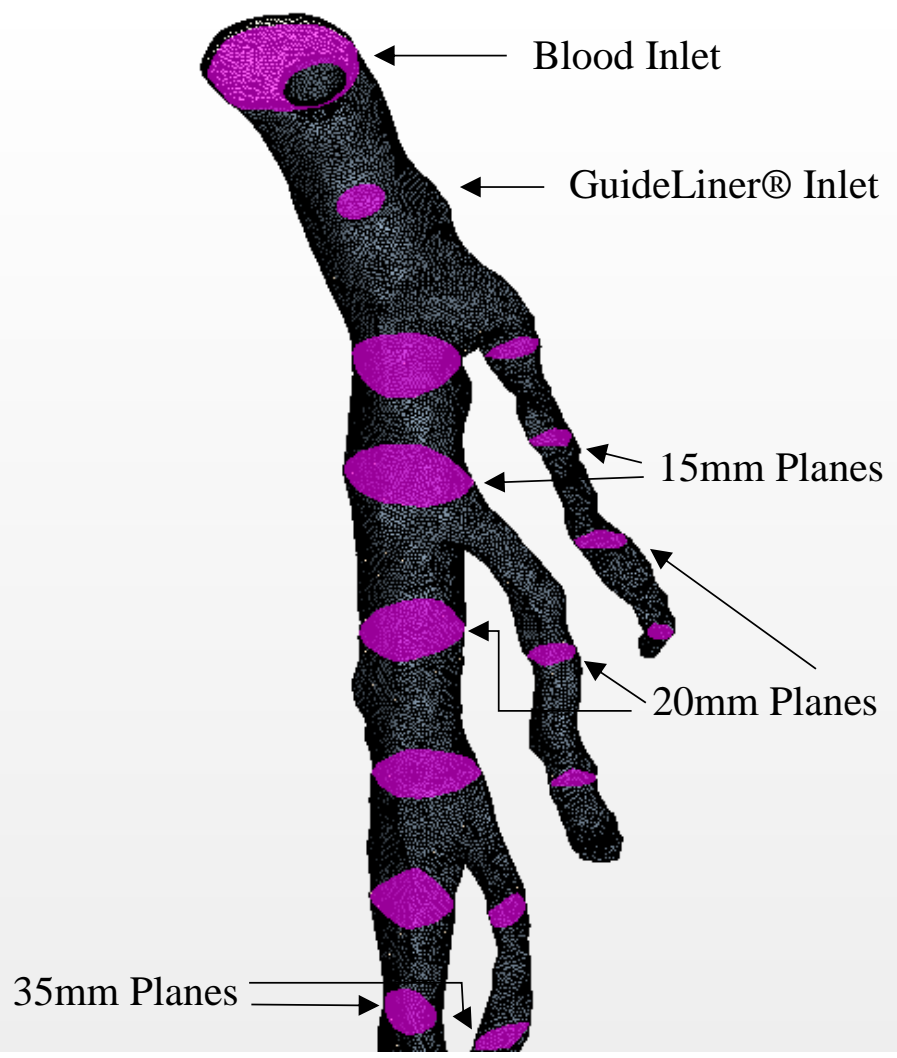

Figure 7.21. Continuation of planes in $5 \mathrm{~mm}$ increments along each bifurcated branch that had deviated from the mother vessel in HH97 LAD.

The CFD simulation was executed for Fig. 7.16 and the ABT's from the GuideLiner® inlet to the cross-sectional plane at $35 \mathrm{~mm}$ were plotted comparing the ABT for the vessel pathway of interest verse the combined ABT for each bifurcated pathway (Fig. 7.22). 


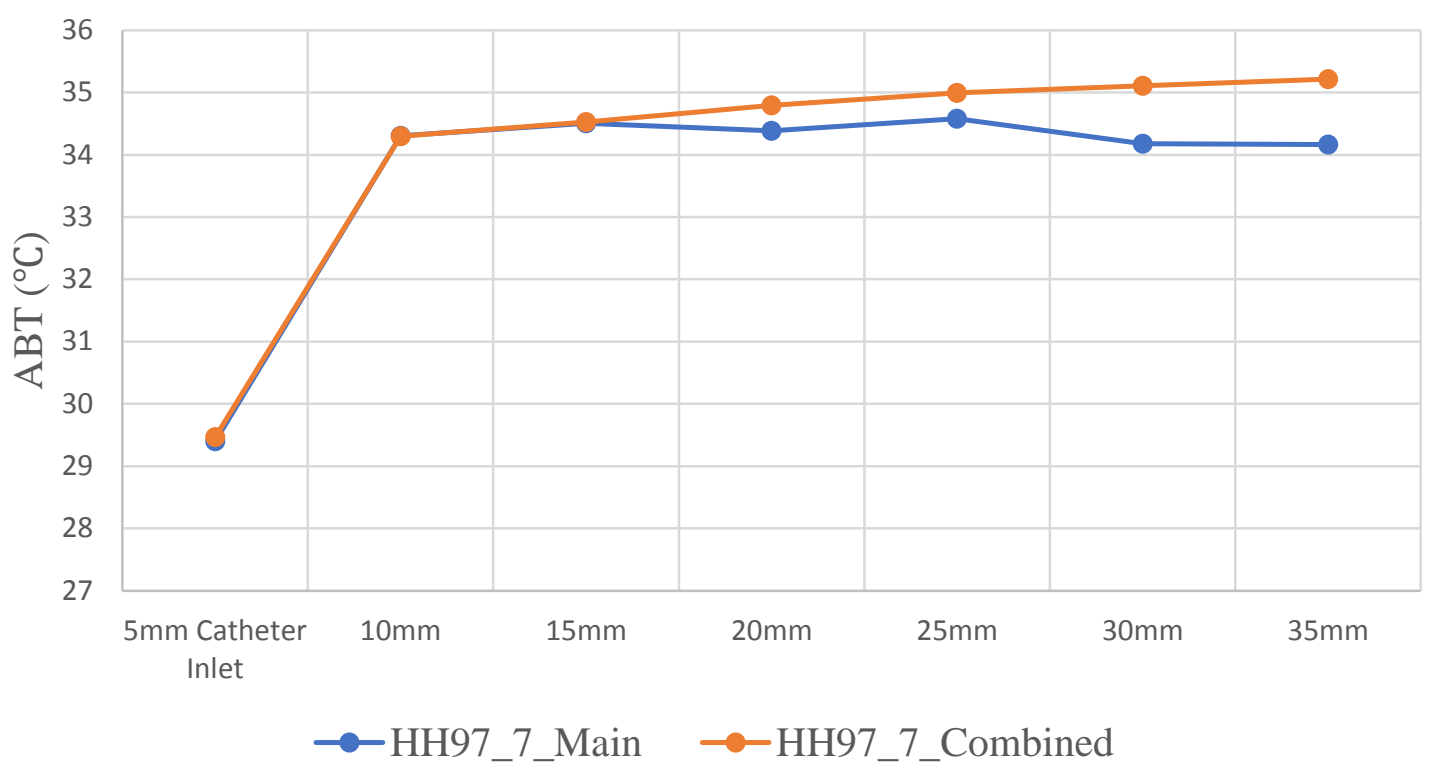

Figure 7.22. HH97 ABT's comparing the vessel pathway of interest vs the combined ABT for all branches between the GuideLiner ${ }^{\circledR}$ inlet and the $35 \mathrm{~mm}$ plane.

Having closely examined the impact of vascular geometry on cooling, at a single flow rate, the next logical exploration involved alternative cooling catheter operating conditions. The 7F guiding catheter and 7F GuideLiner ${ }^{\circledR}$ used in the in vitro mock loop system (see chapter 4) was implemented for the purpose of determining how the guide catheter and GuideLiner® performed in physiological conditions. Guide catheters are manufactured in many different lengths and diameters and have many different functions in cardiovascular procedures [151]. They can be used as conduits for wire transport, contrast injections, and advancing interventional devices. Guide catheters are also known for their high kink resistance, device compatibility, and radiopacity features [151]. A standard Cordis ${ }^{\circledR} 7 \mathrm{~F}$ guide catheter and GuideLiner ${ }^{\circledR}$ was selected for this study and tested in the in vitro mock loop to determine outlet temperatures and corresponding flowrates to be used in computational fluid dynamics (CFD) simulations. A visual representation for each patient-specific LAD (Fig. 7.23) and LCX (Fig. 7.24) network 
was generated to observe the impact of the standard catheter's performance on cooling effectiveness.
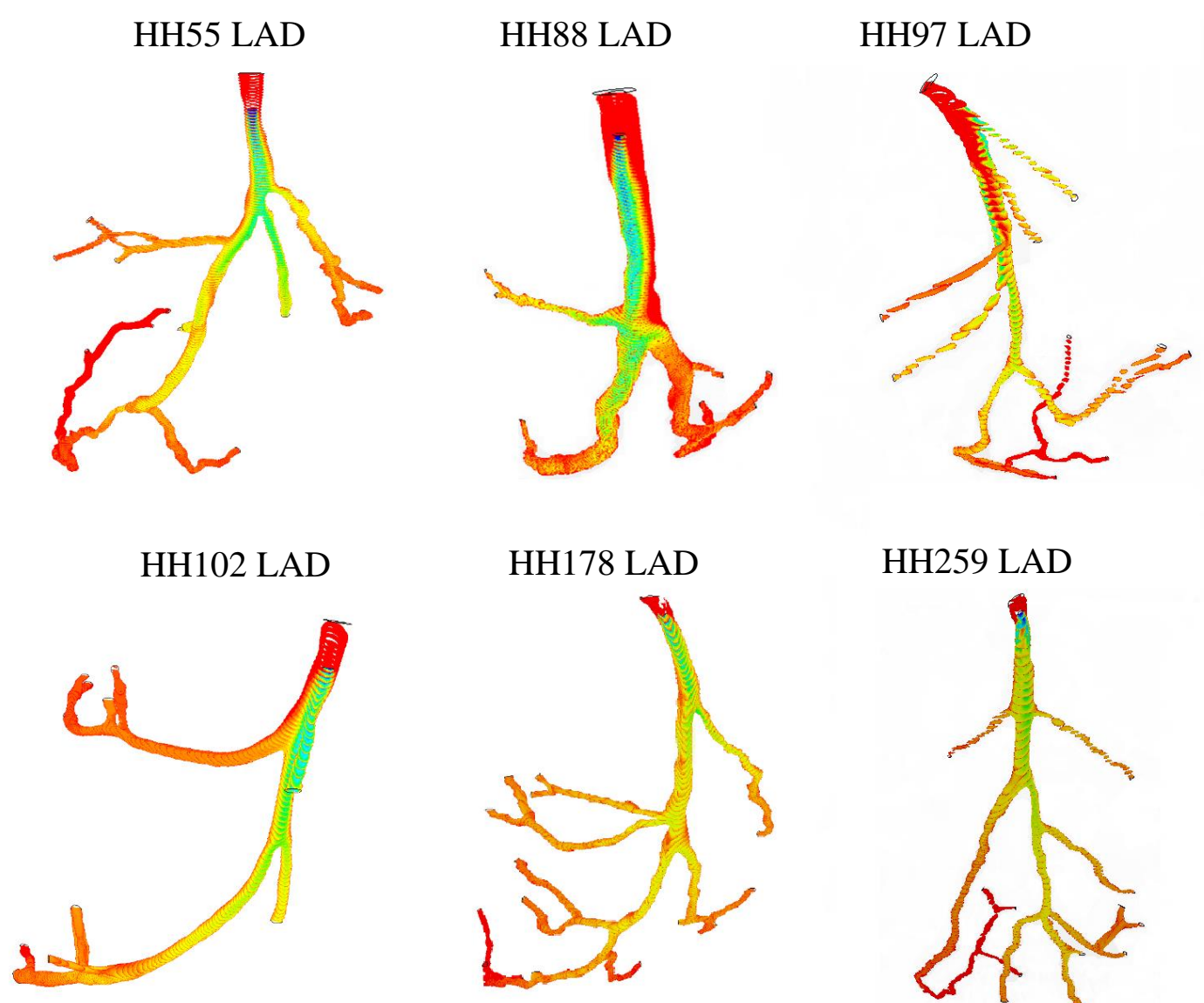

\section{HH259 LAD}

Temperature (C)
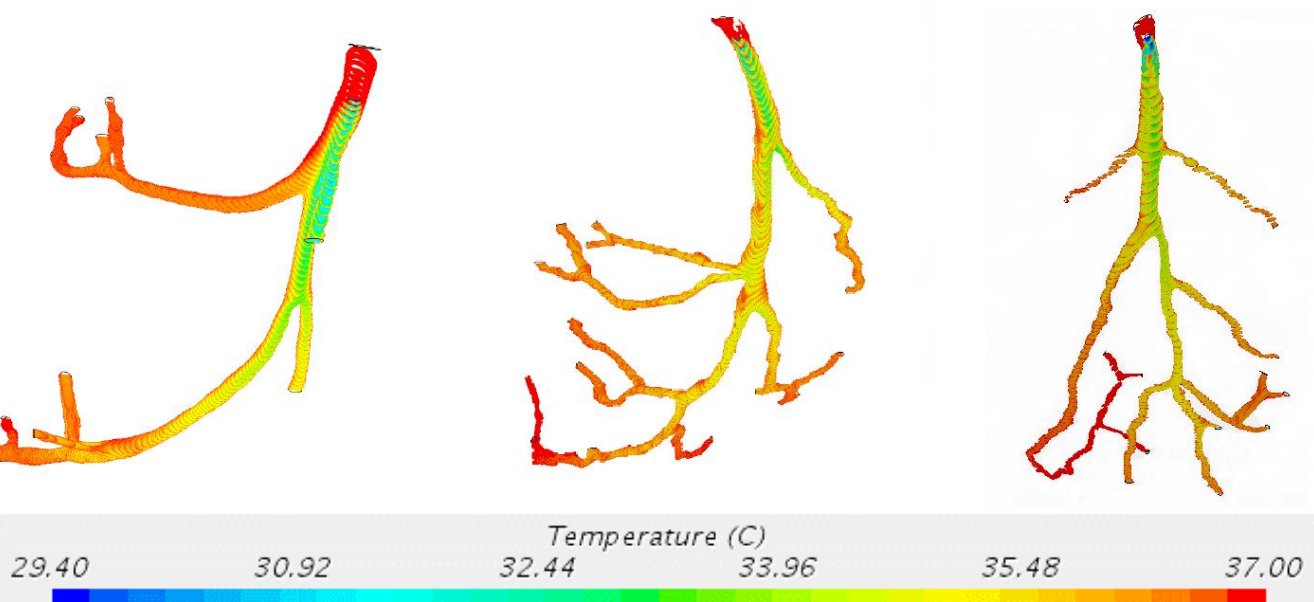

$35.48 \quad 37.00$

Figure 7.23. Temperature distributions executed in STAR-CCM+ for all patient-specific LAD models using a GuideLiner ${ }^{\circledR}$ flow rate of $29.2 \mathrm{ml} / \mathrm{min}$ at $29.4^{\circ} \mathrm{C}$ in all models. 
HH55 LCX

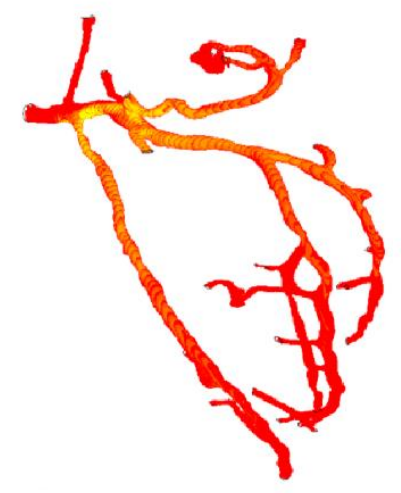

HH102 LCX

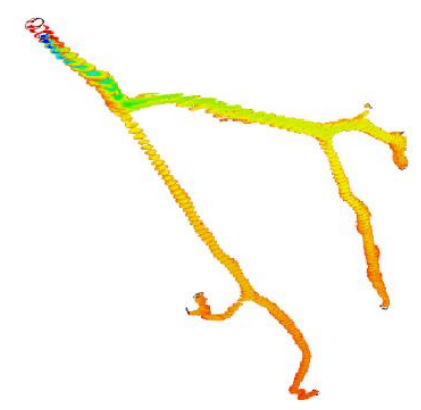

29.40

30.92
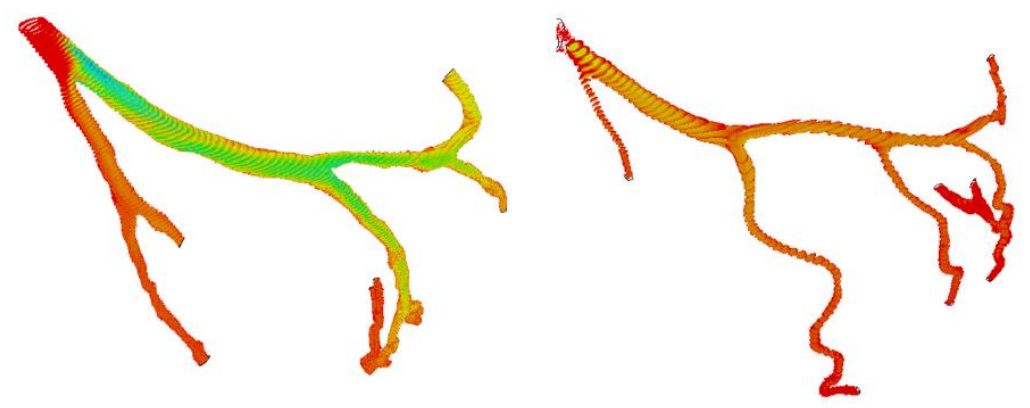

HH178 LCX

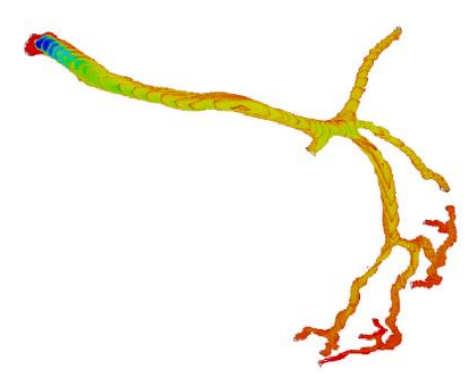

Temperature (C)

32.44

33.96

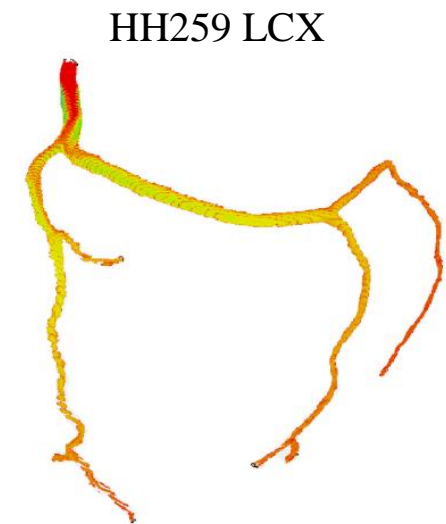

35.48

37.00

Figure 7.24. Temperature distributions executed in STAR-CCM+ for all patient-specific LCX heart models using a GuideLiner® flow rate of $29.2 \mathrm{ml} / \mathrm{min}$ at $29.4^{\circ} \mathrm{C}$ in all models.

Impact of TH device location placement on cooling effectiveness. Localized

TH transcatheter procedures in patient-specific heart vasculature can have varying results on cooling effectiveness (Figs 7.23 and 7.24). In this section, we analyzed the impact of GuideLiner ${ }^{\circledR}$ cooling effectiveness in each patient-specific LAD and LCX vasculature. The tip of the GuideLiner® was positioned in the proximal LAD and LCX for each human heart, and the ABT distributions for HH55 (Fig. 7.25) and HH178 (Fig. 7.26) were compared using flowrates at $29.2 \mathrm{ml} / \mathrm{min}$ and $68.85 \mathrm{ml} / \mathrm{min}$ for each patient. 


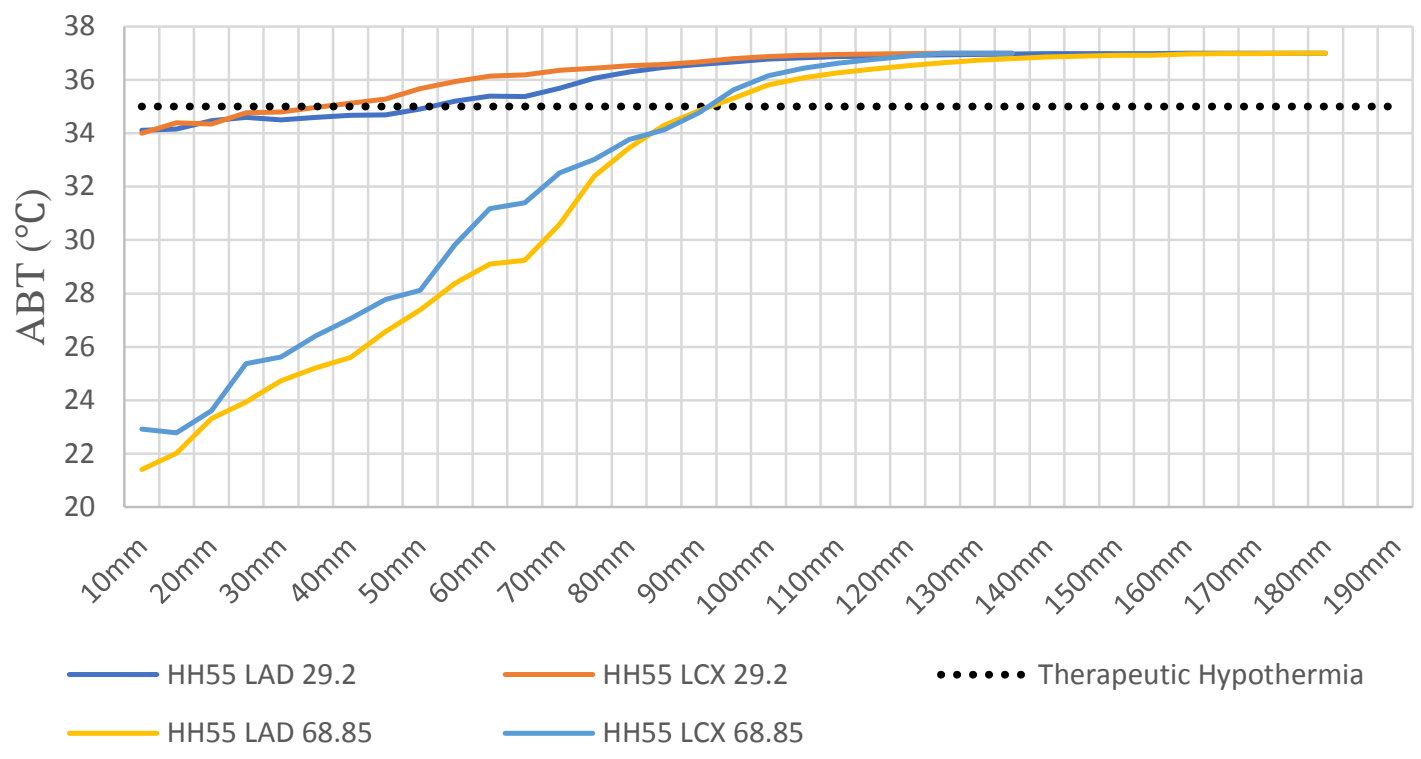

Figure 7.25. Impact of proximal GuideLiner ${ }^{\circledR}$ placement in the LAD and LCX for HH55 using $29.2 \mathrm{ml} / \mathrm{min}$ and $68.85 \mathrm{ml} / \mathrm{min}$ flowrates. ABT's below black-dotted line represent effective therapeutic hypothermia cooling $\left(<35^{\circ} \mathrm{C}\right)$.

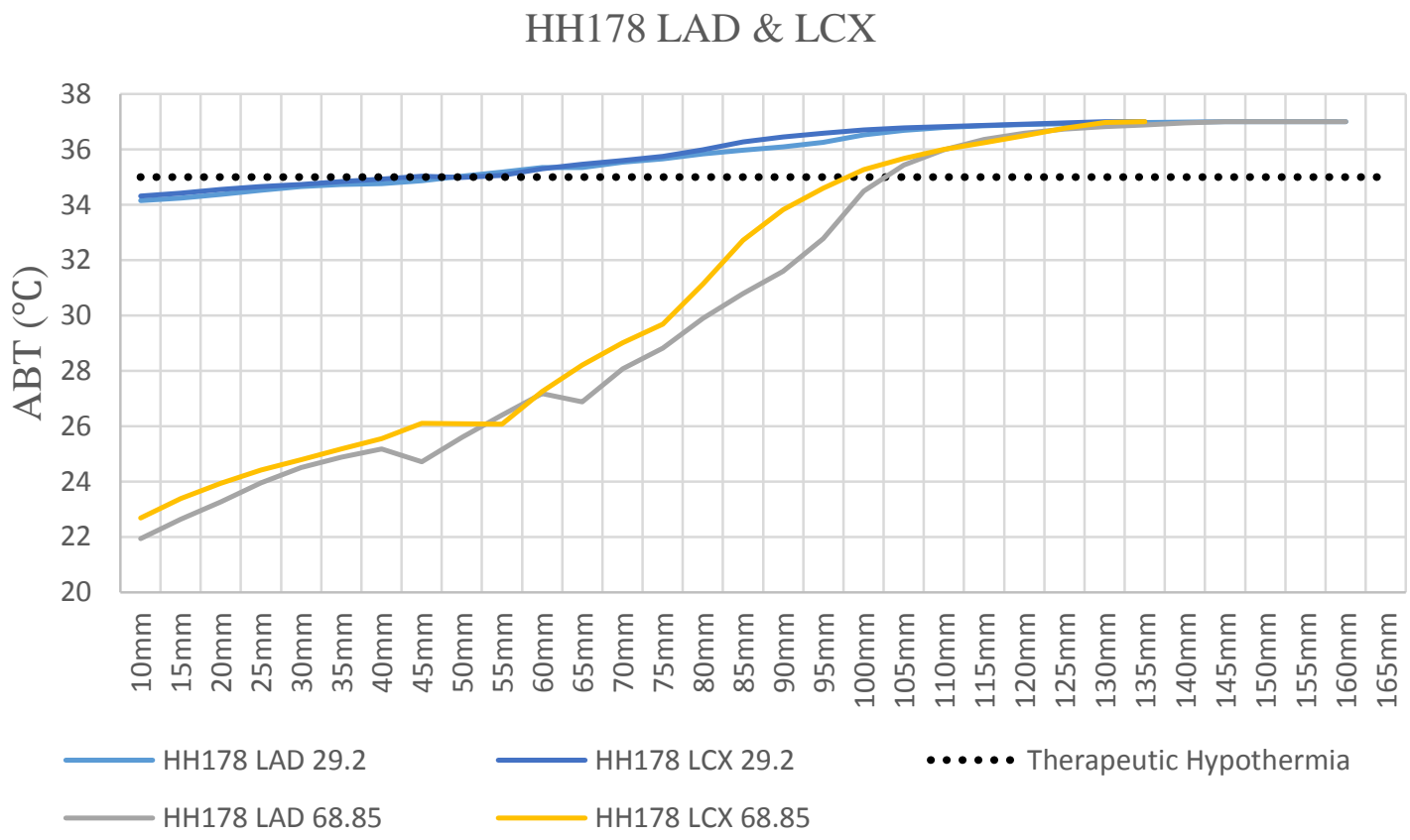

Figure 7.26. Impact of proximal GuideLiner ${ }^{\circledR}$ placement in the LAD and LCX for HH178 using $29.2 \mathrm{ml} / \mathrm{min}$ and $68.85 \mathrm{ml} / \mathrm{min}$ flowrates. ABT's below black-dotted line represent effective therapeutic hypothermia cooling $\left(<35^{\circ} \mathrm{C}\right)$. 
Although identical GuideLiner® flowrates and inlet temperatures were delivered to the proximal end of each LAD and LCX HH model, the assumption that each artery network will experience identical cooling effectiveness was not found. Effective TH temperatures were evident in all LAD and LCX networks, however, the distance by which TH was effective varied between patients (Table 7.1).

Table 7.1

Summary of TH cooling effectiveness for all HH patients using $29.2 \mathrm{ml} / \mathrm{min}$ and 68.85 $\mathrm{ml} / \mathrm{min}$ GuideLiner ${ }^{\circledR}$ flowrates.

Effective TH-ABT at $29.2 \mathrm{ml} / \mathrm{min}$

\section{Human Heart}

HH55

HH88

HH97

HH102

HH178

HH259
LAD

$50 \mathrm{~mm}$

$95 \mathrm{~mm}$

$55 \mathrm{~mm}$

$35 \mathrm{~mm}$

$45 \mathrm{~mm}$

$35 \mathrm{~mm}$

\section{LCX}

$35 \mathrm{~mm}$

$60 \mathrm{~mm}$

$55 \mathrm{~mm}$

$50 \mathrm{~mm}$

$50 \mathrm{~mm}$

$35 \mathrm{~mm}$

Note. Therapeutic hypothermia average bulk temperature (TH-ABT) represents the distance from the inlet up to and including the plane listed where $\mathrm{TH}$ temperature conditions exist $\left(<35^{\circ} \mathrm{C}\right)[152]$. The greater-than sign obverse to the distance planes signifies that the planes reached the artery outlet, and that effective TH-ABT's continued past the outlet.

Based on compact heat exchanger design, surface area to fluid volume ratio is a logical parameter to examine for intravascular cooling. Surface area to volume (SA:V) ratios were calculated in Chapter 5 (Fig. 5.6) for all LAD and LCX RH models. The goal of calculating SA:V ratios was to correlate their impact on heart cooling effectiveness levels. The SA:V ratios were grouped in ascending order and plotted vs effective THABT distance for each corresponding flow rate in the LAD (Fig. 7.27) and LCX (Fig. 7.28) arteries. 
LAD Flowrates vs Effective TH-ABT $\left(<35^{\circ} \mathrm{C}\right)$

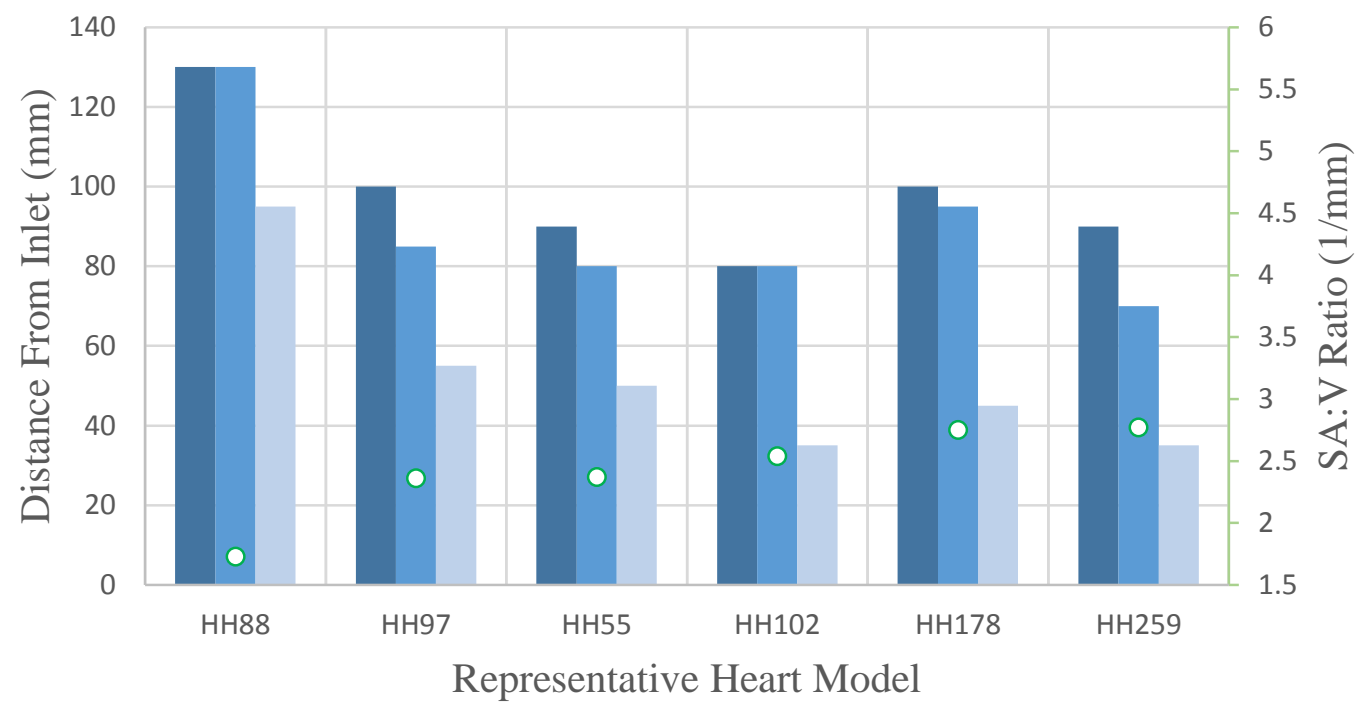

$\square 68.85 \mathrm{ml} / \mathrm{min} \quad \square 9.03 \mathrm{ml} / \mathrm{min} \quad 29.2 \mathrm{ml} / \mathrm{min} \quad$ OSA:V Ratio

Figure 7.27. Impact of surface area to volume (SA:V) ratios on cooling effectiveness in the LAD pathway of interest for each representative heart $(\mathrm{RH})$ model. Cooling effectiveness is the ratio of the length of TH-ABT in the mother vessel over the entire length of the mother vessel. Therapeutic hypothermia average bulk temperature (TH$\mathrm{ABT}$ ) represents the distance from the inlet up to and including the plane listed where $\mathrm{TH}$ temperature conditions exist $\left(<35^{\circ} \mathrm{C}\right)$. In general, smaller $\mathrm{SA}: \mathrm{V}$ ratios lead to deeper cooling, as would be expected. Note: SA:V ratio values are listed from left to right in ascending order. 
LCX Flowrates vs Effective TH-ABT $\left(<35^{\circ} \mathrm{C}\right)$

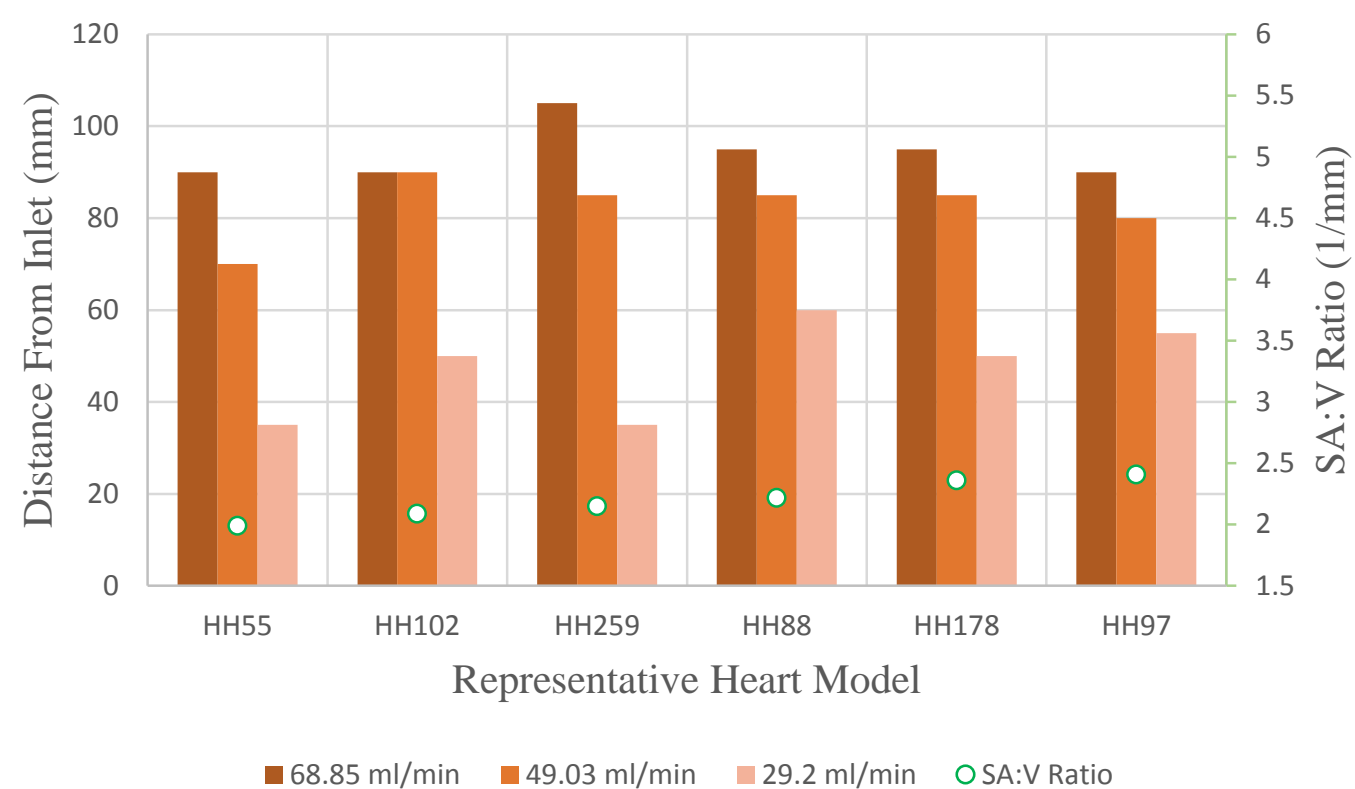

Figure 7.28. Impact of surface area to volume (SA:V) ratios on cooling effectiveness in the LCX pathway of interest for each representative heart (RH) model. Cooling effectiveness is the ratio of the length of TH-ABT in the mother vessel over the entire length of the mother vessel. Therapeutic hypothermia average bulk temperature $\mathrm{TH}-$ $\mathrm{ABT}$ ) represents the distance from the inlet up to and including the plane listed where $\mathrm{TH}$ temperature conditions exist $\left(\angle 35^{\circ} \mathrm{C}\right)$. In general, smaller $\mathrm{SA}: \mathrm{V}$ ratios lead to deeper cooling, as would be expected. Note: SA:V ratio values are listed from left to right in ascending order.

Figures 7.27 and 7.28 reveal the impact of SA:V ratios, listed from left to right in ascending order, on cooling effectiveness in the fluid pathway of interest for all LAD and LCX artery models. Three GuideLiner® flowrates at $29.2 \mathrm{ml} / \mathrm{min}, 49.03 \mathrm{ml} / \mathrm{min}$, and $68.85 \mathrm{ml} / \mathrm{min}$ were infused eccentrically at each proximal inlet and compared to the max distance in the pathway of interest where ABT's contained $\mathrm{TH}\left(<35^{\circ} \mathrm{C}\right)$ temperatures.

LAD artery simulations for RHs HH55, HH88, $\mathrm{HH} 97$, and $\mathrm{HH} 102$ revealed a direct correlation between effective $\mathrm{TH}$ cooling and low SA:V ratio values for all three flowrates. However, LCX artery results for RHs HH88, HH97, HH178, and HH259 
revealed a direct correlation between effective $\mathrm{TH}$ cooling and low SA:V ratio values at $49.03 \mathrm{ml} / \mathrm{min}$ and $68.85 \mathrm{ml} / \mathrm{min}$ flowrates.

As mentioned in Chapter 5, SA:V ratios are small in artery networks with enlarged internal volumes [142]. This implies that TH cooling will be conserved downstream since blood flow temperatures drastically differ from surrounding tissue when moderately large vessels experience blood flow [149]. Small SA:V ratios reveal that for every unit of volume, there is less surface area to exchange heat. If a vascular network maintains larger diameters directly near or near distal to the guide extrusion tip, the cooling effect will travel deeper in the vessel. Therefore, small SA:V ratios imply larger vessels.

A sensitivity analysis for proximal LAD and LCX lengths with respect to cooling effectiveness was determined for the $68.85 \mathrm{~mL} / \mathrm{min}$ GuideLiner ${ }^{\circledR}$ flow rates. Figures 7.29 and 7.30 illustrate the sensitivity of proximal length to the total mother vessel length for each LAD and LCX artery simulation. 
Sensitivity of LAD Proximal Length to Mother Vessel Length

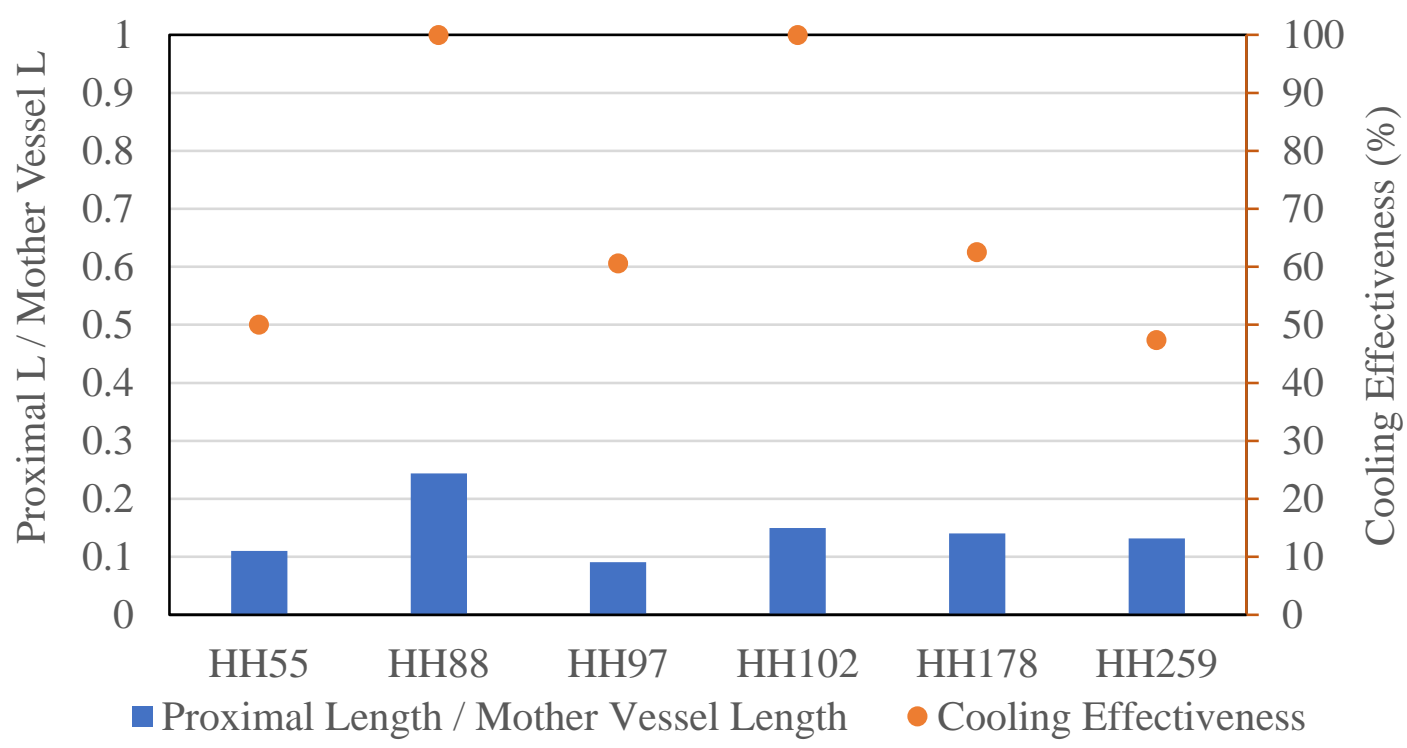

Figure 7.29. Sensitivity of proximal length to mother vessel length for each HH LAD model. Proximal length revealed significant sensitivity to cooling effectiveness in HH88 and $\mathrm{HH} 102$, as the two longest proximal length revealed the greatest cooling effectiveness. However, no significant sensitivity was determined between the remaining $\mathrm{HH}$ models.

Sensitivity of LCX Proximal Length to Mother Vessel Length

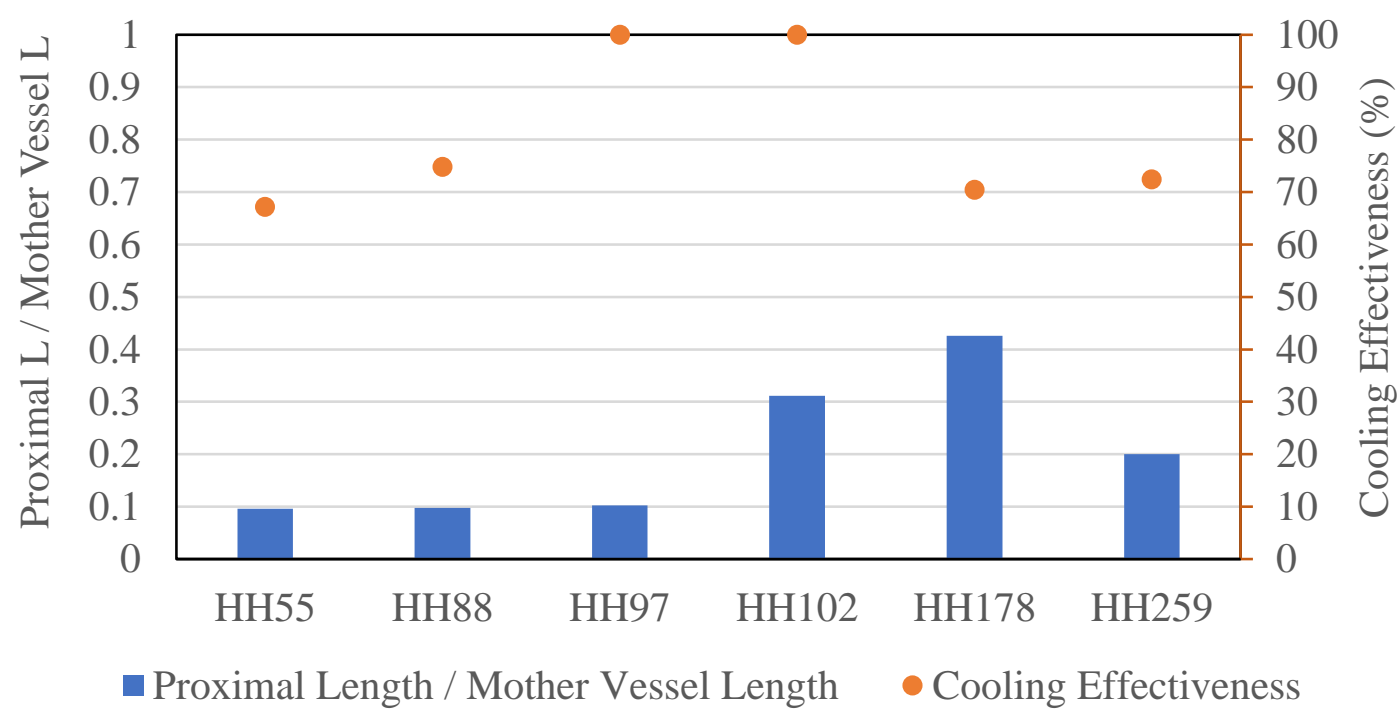

Figure 7.30. Sensitivity of proximal length to mother vessel length for each HH LCX model. Proximal length revealed no significant sensitivity to cooling effectiveness since the longest (HH178) and shortest (HH55) proximal artery lengths played very little impact on cooling effectiveness, as compared to HH97 and HH102. 
Cooling effectiveness in each mother vessel was illustrated for the LAD (Fig. 7.29) and LCX (Fig. 7.30). Proximal artery lengths ranged between $12 \mathrm{~mm}$ to $31.7 \mathrm{~mm}$ in the LAD and $9.23 \mathrm{~mm}$ to $57.5 \mathrm{~mm}$ in the LCX. The sensitivity of the proximal artery lengths to the total mother vessel lengths provided no significant correlation to cooling effectiveness for all $\mathrm{HH}$ models.

To increase the rigor of our analyses, the entire vascular networks were characterized to determine the impact of cooling effectiveness down each first and second obtuse marginal branch and posterolateral branch in the LCX, and down each first and second diagonal branch, septal branch, and anterior descending branch in the LAD. Total cooling in the vascular network was determined using cooling effectiveness from each daughter vessel as described by the following equation:

$$
\eta_{T C}=\frac{\sum_{i=1}^{n} \cdot \frac{L_{e}}{L}}{N}
$$

where $\eta_{T C}$ is total cooling in the vascular network $\left(\eta_{T C}=1\right.$ represents $\left.100 \% \mathrm{CE}\right), L_{e}$ is the effective length in each branch of interest where $\mathrm{TH}-\mathrm{ABT} s<35^{\circ} \mathrm{C}, \mathrm{L}$ is the total length of each branch of interest, and $N$ represents the sum of the total number of branches in the network of interest.

A visual representation of Eq. 7.1 applied to the LCX artery of patient's HH88, HH97, and HH259 is illustrated in Figure 7.31. 
HH88 LCX

$\eta_{T C}=85.26 \%$

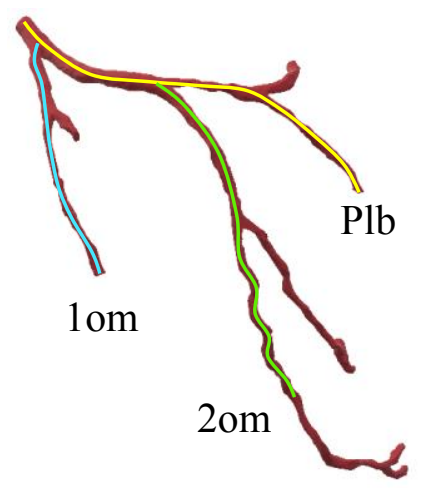

\begin{tabular}{|c|c|}
\hline Branch & $\frac{\boldsymbol{L}_{\boldsymbol{e}}}{\boldsymbol{L}}$ \\
\hline $10 \mathrm{~m}$ & $100 \%$ \\
\hline $2 \mathrm{om}$ & $65.5 \%$ \\
\hline Plb & $100 \%$ \\
\hline
\end{tabular}

HH97 LCX

$\eta_{T C}=97.63 \%$

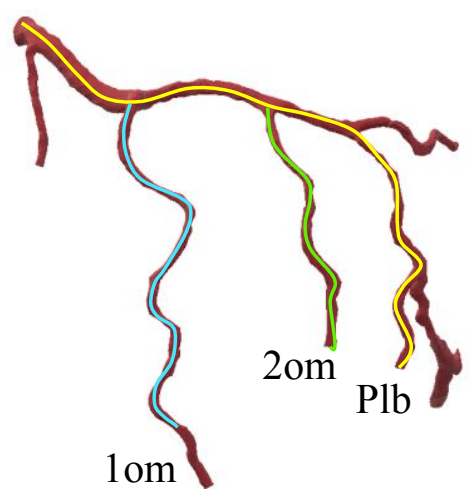

\begin{tabular}{|c|c|}
\hline Branch & $\frac{\boldsymbol{L}_{\boldsymbol{e}}}{\boldsymbol{L}}$ \\
\hline 1 om & $92.70 \%$ \\
\hline $2 \mathrm{om}$ & $100 \%$ \\
\hline Plb & $100 \%$ \\
\hline
\end{tabular}
HH259 LCX

$\eta_{T C}=76.02 \%$

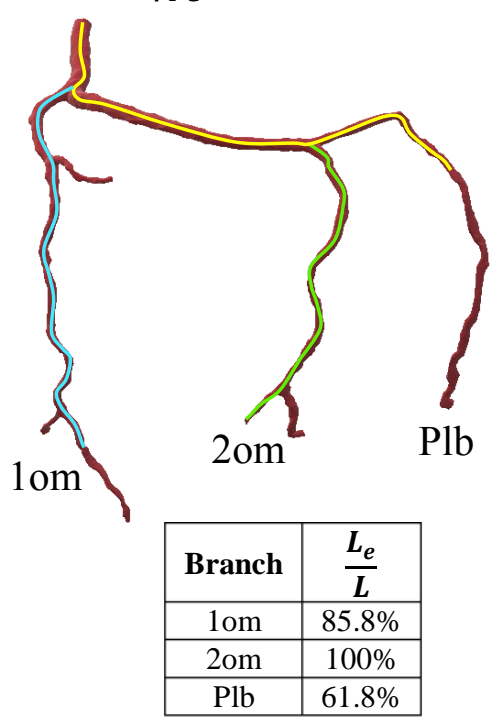

Figure 7.31. Characterization of vascular networks for HH88, HH97, and HH259 LCX represented using $68.85 \mathrm{~mL} / \mathrm{min}$ GuideLiner® flow rates. First obtuse marginal (1om), second obtuse marginal (2ob), and posterolateral (Plb) branches were characterized using Eq. 7.1. Total cooling $\eta_{T C}$ in HH97 resulted in $97.63 \%$ CE in the artery network, while $\mathrm{HH} 88$ and $\mathrm{HH} 259$ resulted in $85.26 \%$ and $76.02 \%$ total $\mathrm{CE}$ in each vascular network, respectively.

We acknowledge this method is subjective, and intend that it be used as a simple aid to help and support patient care. The purpose of the proposed method is to help stimulate a deeper understanding of intracoronary cooling and provide a deeper understanding of TH treatment methods to improve patient outcomes following AMI. The HH97 LCX artery network resulted in having 97.63\% CE in its total vascular network, and would be recommended as a good candidate for localized cooling therapy. On the other hand, we would be reluctant to recommend HH88 and HH259 LCX arteries as candidates for this treatment due to their moderate total network cooling potential of $85.26 \%$ and $76.02 \%$, respectively.

The total network cooling effectiveness for patients HH55 and HH178 was not included in the following analyses due to poor mother vessel and total network cooling 
performance. The remaining patient models provided good cooling performances and were analyzed using a dimensionless parameter of SA:V multiplied by total network length. These values were plotted against $\eta_{T C}$ and mother vessel cooling effectiveness for each LAD and LCX network using the $68.85 \mathrm{~mL} / \mathrm{min}$ GuideLiner® inlet flow rate (Fig. 7.32).

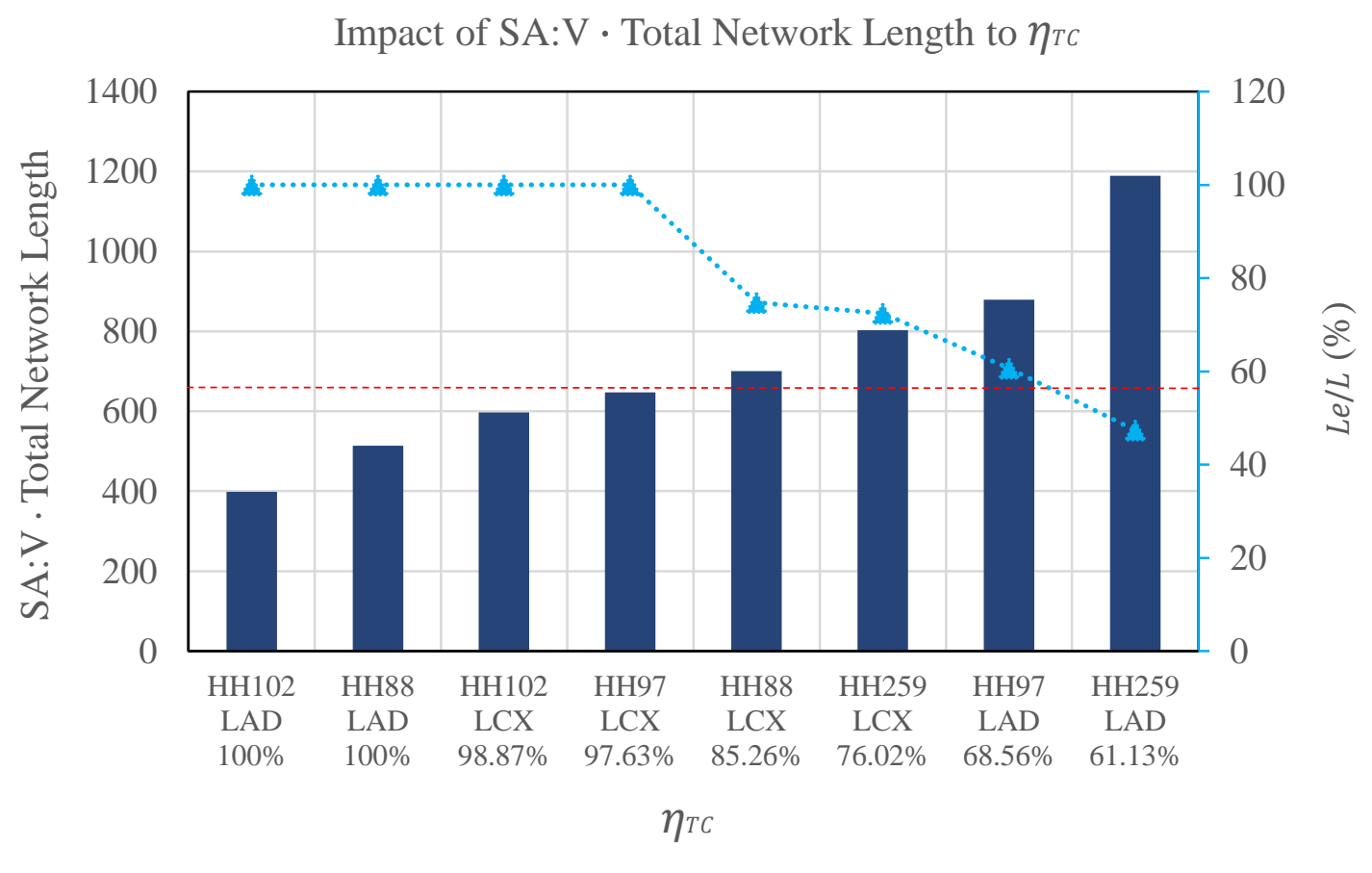

Figure 7.32. Analyses of total network cooling effectiveness $\left(\eta_{T C}\right)$ for each $\mathrm{HH}$ model compared to $\mathrm{SA}: \mathrm{V} \cdot$ total network length. Triangle markers represent mother vessel cooling effectiveness $(L e / L)$ for each $\mathrm{HH}$ model at $68.85 \mathrm{~mL} / \mathrm{min}$ GuideLiner® flow rate, while circles represent $\mathrm{SA}: \mathrm{V} \cdot$ total network length for each $\mathrm{HH}$ model. A significant reduction in total network cooling effectiveness is evident when $\mathrm{SA}: \mathrm{V} \cdot$ total network length ratios exceed 700. Note: red dotted line represents 700 cut-off value between good and bad heart cooling candidates.

Total network cooling effectiveness is significantly reduced when SA:V • total network length ratios exceed 700 . The point where these values exceed 700 is where $\eta_{T C}$ 
is reduced from $97.63 \%$ to $85.26 \%$. This is also indicative of the fact that mother vessel cooling effectiveness also followed suit by decreasing from $100 \%$ to $74.8 \%$. The comparison between these two trends suggest that a cutoff of $700 \mathrm{SA}: \mathrm{V} \cdot$ total network length ratio could be approximated as a reasonable localized cooling candidate parameter. However, this assumption is limited by the number of patient-specific vascular models employed in this study. A more robust analysis is needed to increase the fidelity of this assumption by improving upon the modeling physics of the CFD simulations and broadening the patient-specific model population.

In lieu of the importance of these findings and insights attained from this study, a comparison of $\mathrm{HH}$ specifications and published works must also be made to preserve the fidelity of this work.

\section{Comparison with Published Works}

Patient-specific heart specifications, medical history, and intracoronary temperature predictions were compared with published works that performed similar localized cooling studies in the coronary arteries (Table 7.2) [13][96]. 
Table 7.2

Comparison of patient specifications and medical history with patients in similar heart cooling studies.

\begin{tabular}{|c|c|c|c|}
\hline Comparison of Work & Present Work & Otterspoor [13] & McGarvey [96] \\
\hline $\begin{array}{c}\text { Intracoronary Temperature } \\
\text { Goal }\end{array}$ & $\begin{array}{l}<35^{\circ} \mathrm{C} \text { at each } \\
\text { artery outlet }\end{array}$ & $\begin{array}{l}5^{\circ} \mathrm{C} \text { below body } \\
\text { temp. proximal } \\
\text { to occlusion }\end{array}$ & $\begin{array}{l}3.4^{\circ} \mathrm{C} \text { below body } \\
\text { temp. proximal to } \\
\text { occlusion }\end{array}$ \\
\hline Patient population & $\mathrm{N}=6$ & $\mathrm{~N}=10$ & $\mathrm{~N}=10$ \\
\hline Cooling Location & $\begin{array}{l}\operatorname{LAD}(\mathrm{N}=6) \\
\operatorname{LCX}(\mathrm{N}=6)\end{array}$ & $\begin{array}{l}\operatorname{LAD}(\mathrm{N}=6) \\
\operatorname{LCX}(\mathrm{N}=1) \\
\operatorname{RCA}(\mathrm{N}=3)\end{array}$ & $\begin{array}{c}\operatorname{LAD}(\mathrm{N}=3) \\
\text { Mid-LAD }(\mathrm{N}=2) \\
\operatorname{LCX}(\mathrm{N}=1) \\
\operatorname{RCA}(\mathrm{N}=4)\end{array}$ \\
\hline Male & $\mathrm{N}=3$ & $\mathrm{~N}=7$ & $\mathrm{~N}=9$ \\
\hline Female & $\begin{array}{c}\mathrm{N}=2 \\
(1 \text { unknown) }\end{array}$ & $\mathrm{N}=3$ & $\mathrm{~N}=1$ \\
\hline Age & $\begin{array}{c}41 \pm 27 \\
(1 \text { unknown })\end{array}$ & $61 \pm 11$ & $55 \pm 8$ \\
\hline \multicolumn{4}{|l|}{ Medical History } \\
\hline Diabetes Mellitus & $\mathrm{N}=1$ & $\mathrm{~N}=3$ & $\mathrm{~N}=2$ \\
\hline Smoking & $\mathrm{N}=1$ & $\mathrm{~N}=5$ & $\mathrm{~N}=6$ \\
\hline \multicolumn{4}{|l|}{ Cardiac Medical History } \\
\hline Cardiac Arrest & $\mathrm{N}=1$ & --- & --- \\
\hline Hypertension & $\mathrm{N}=2$ & $\mathrm{~N}=5$ & $\mathrm{~N}=2$ \\
\hline Sinus Bradycardia & $\mathrm{N}=2$ & --- & --- \\
\hline
\end{tabular}

Note. Left anterior descending artery (LAD), left circumflex (LCX), and right coronary artery (RCA).

Hypothermia trials have found that patients who undergo therapeutic hypothermia treatments with temperatures $<35^{\circ} \mathrm{C}$ prior to reperfusion result in reduced infarct sizes [152]. Figures 7.33 to 7.39 illustrate the structural-thermal relationship of preferred cooling candidates with respect to flow rate. 


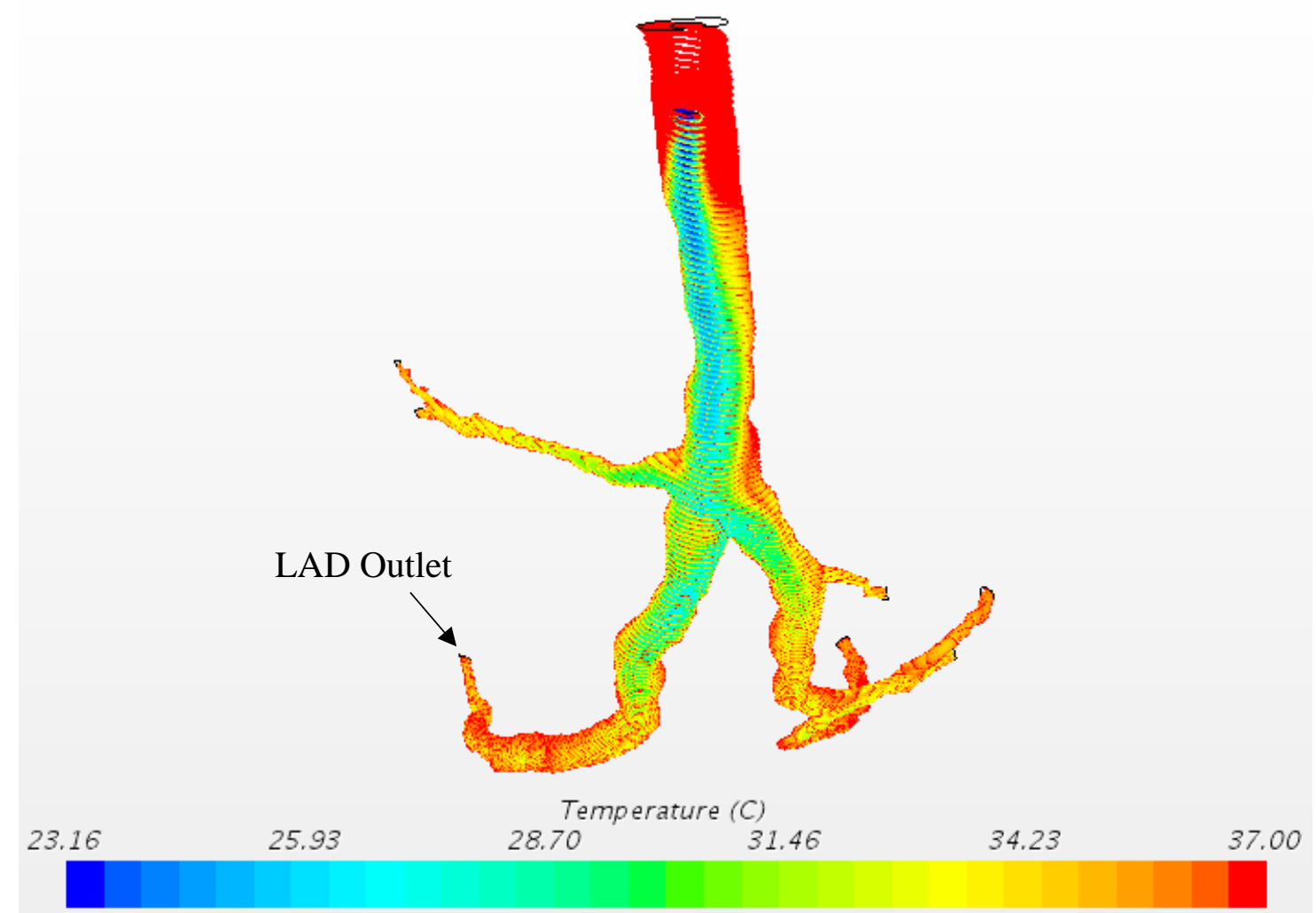

Figure 7.33. Structural-thermal relationship in HH88 LAD for $49.03 \mathrm{ml} / \mathrm{min}$ guide extension flow rate. Note proximal guide extension inlet temperature is $23.16^{\circ} \mathrm{C}$, and LAD outlet temperature is $34.13^{\circ} \mathrm{C}$ 


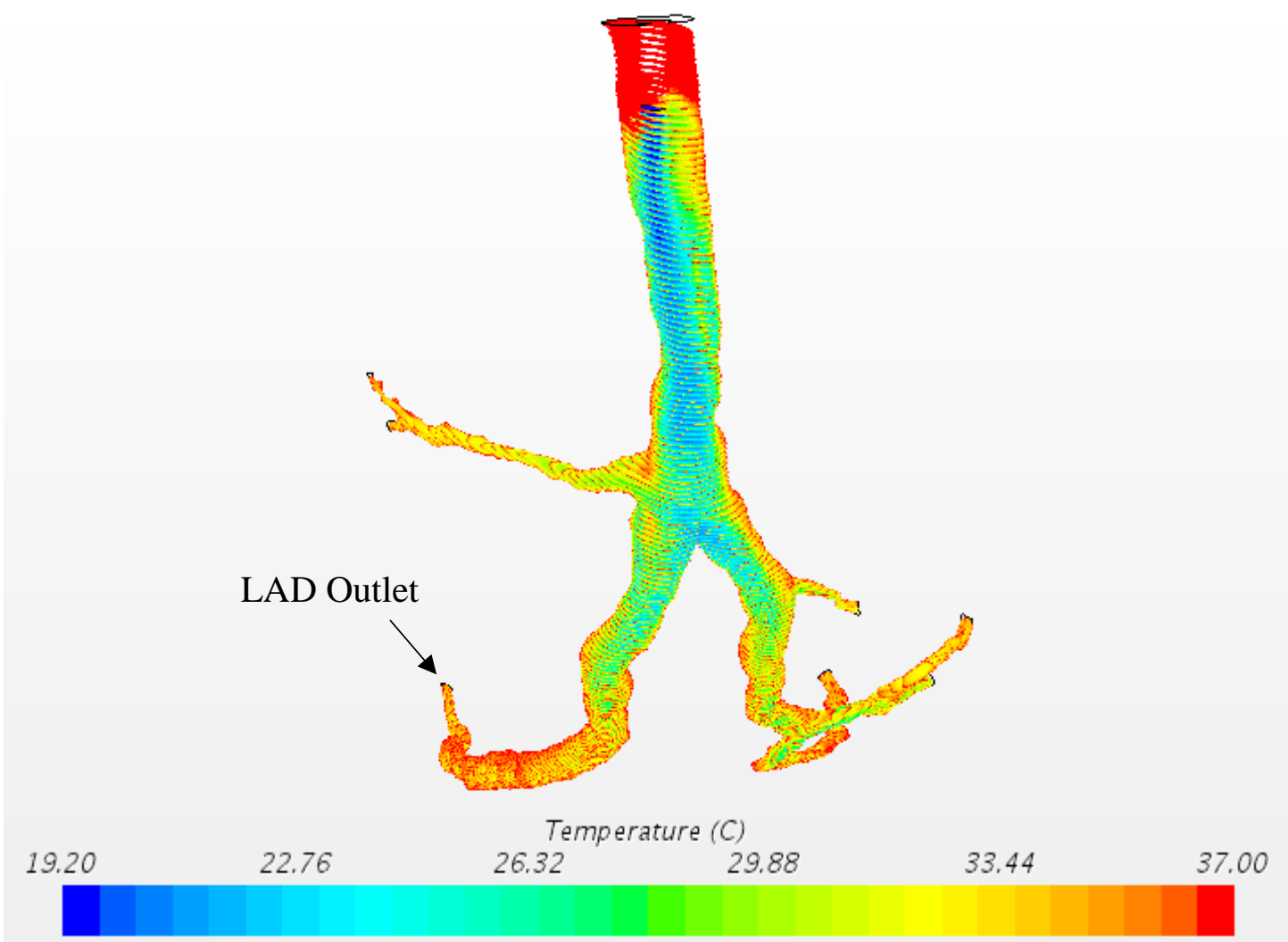

Figure 7.34. Structural-thermal relationship in HH88 LAD for $68.85 \mathrm{ml} / \mathrm{min}$ guide extension flow rate. Note: proximal guide extension inlet temperature is $19.2^{\circ} \mathrm{C}$, and LAD outlet temperature is $32.69^{\circ} \mathrm{C}$. 


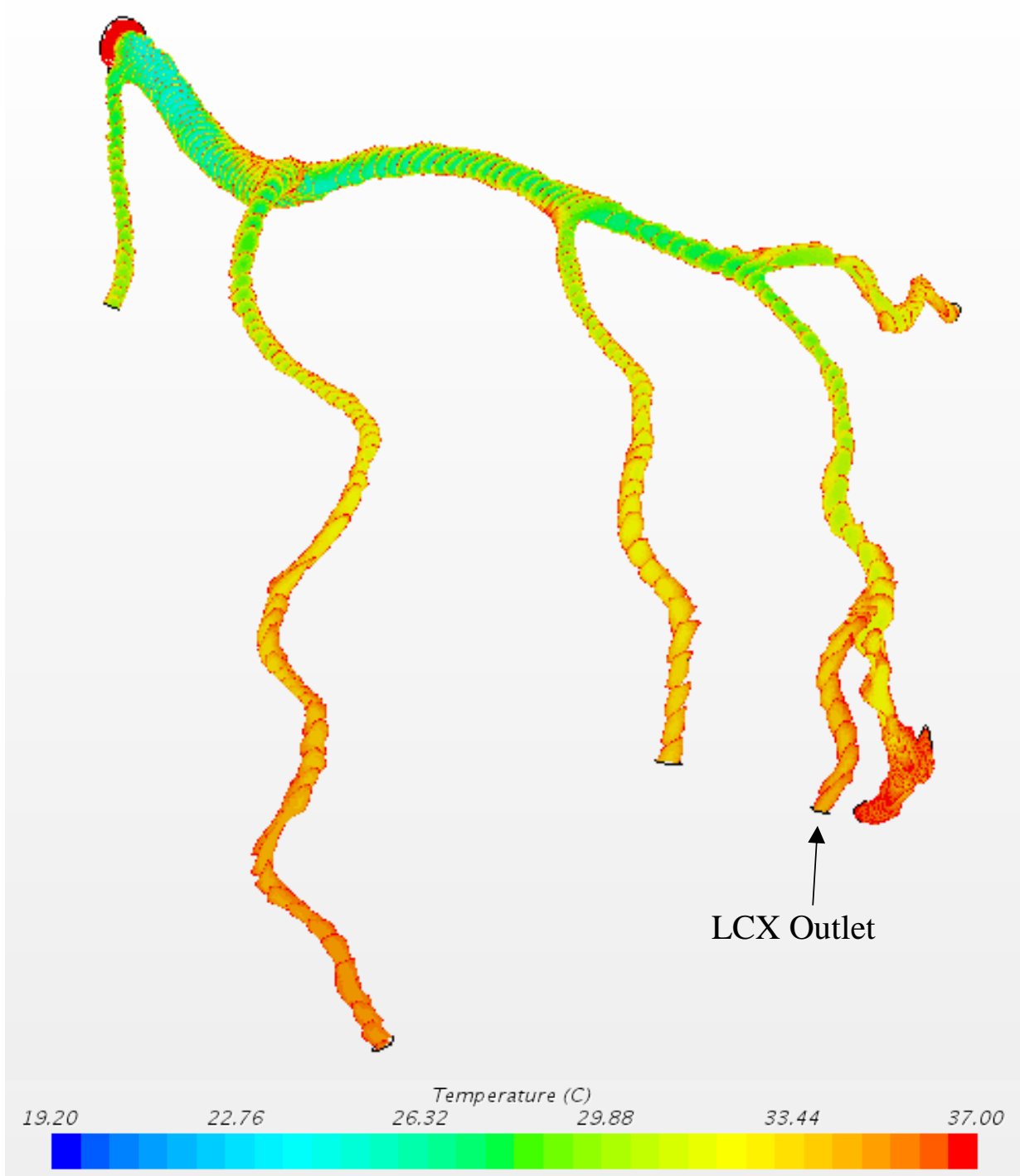

Figure 7.35. Structural-thermal relationship in HH97 LCX for $68.85 \mathrm{ml} / \mathrm{min}$ guide extension flow rate. Note: proximal guide extension inlet temperature is $19.2^{\circ} \mathrm{C}$, and $\mathrm{LCX}$ outlet temperature is $34.55^{\circ} \mathrm{C}$. 


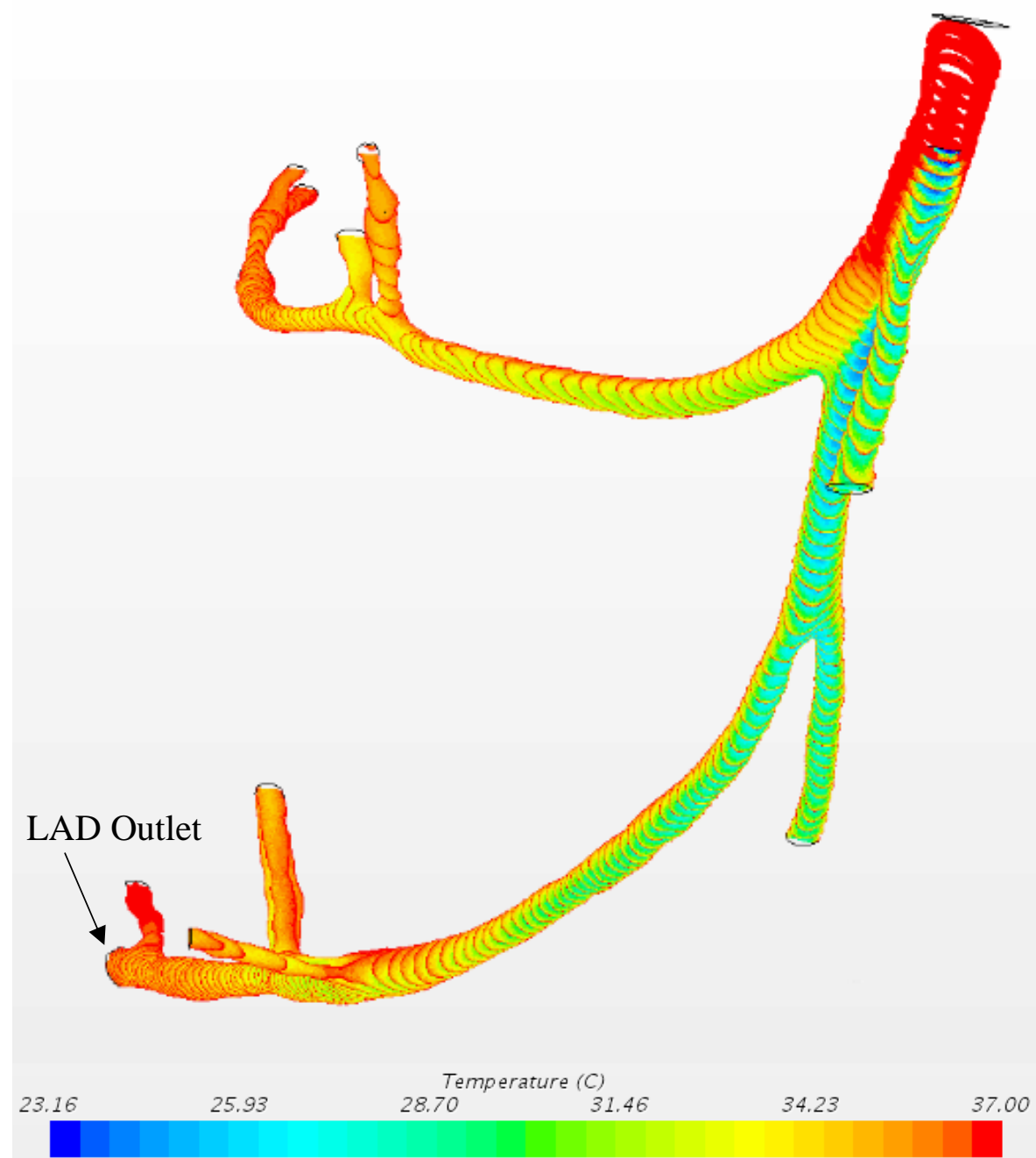

Figure 7.36. Structural-thermal relationship in HH102 LAD for $49.03 \mathrm{ml} / \mathrm{min}$ guide extension flow rate. Note proximal guide extension inlet temperature is $23.16^{\circ} \mathrm{C}$, and LAD outlet temperature is $33.1^{\circ} \mathrm{C}$ 


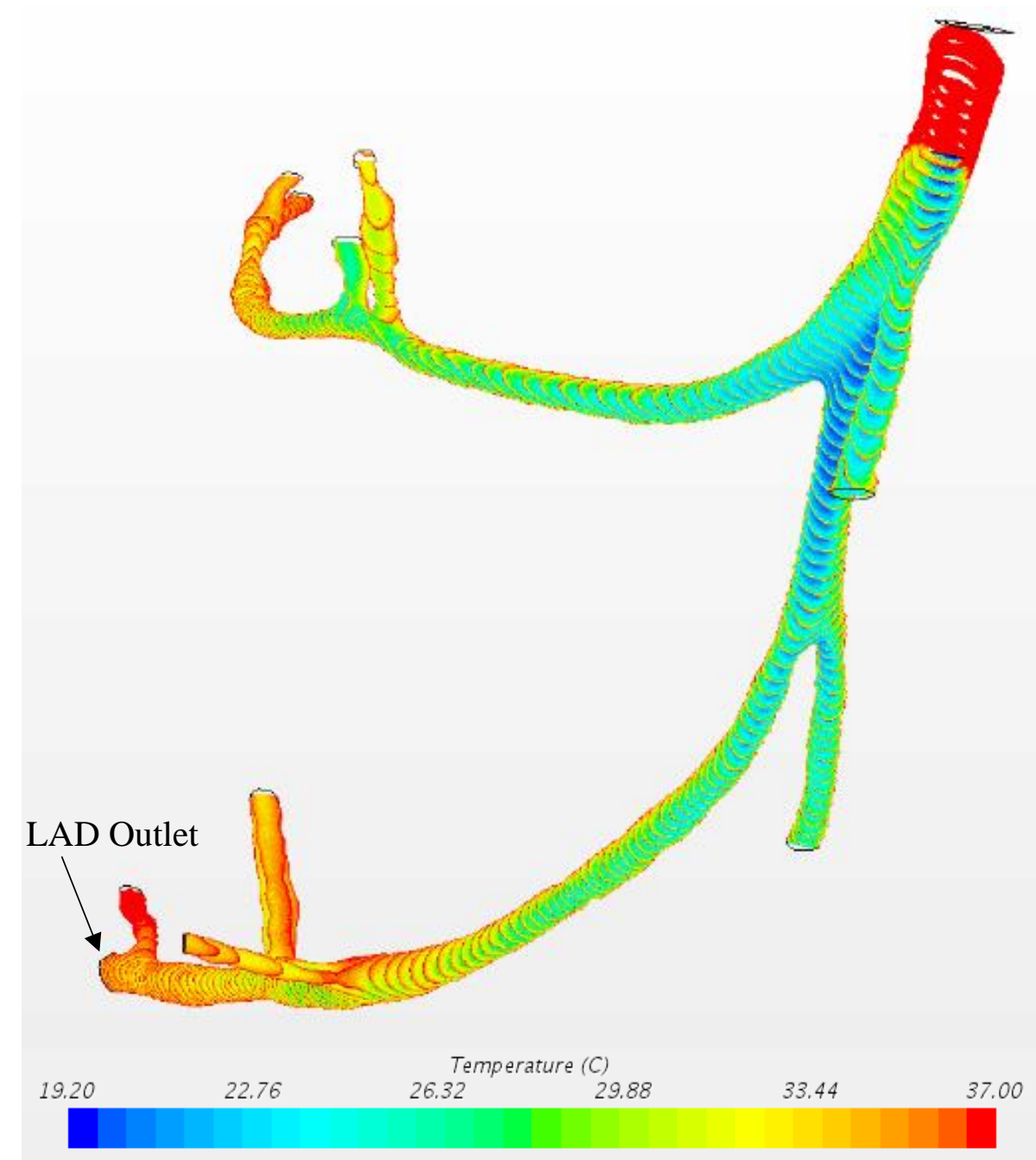

Figure 7.37. Structural-thermal relationship in HH102 LAD for $68.85 \mathrm{ml} / \mathrm{min}$ guide extension flow rate. Note: proximal guide extension inlet temperature is $19.2^{\circ} \mathrm{C}$, and LAD outlet temperature is $30.47^{\circ} \mathrm{C}$. 


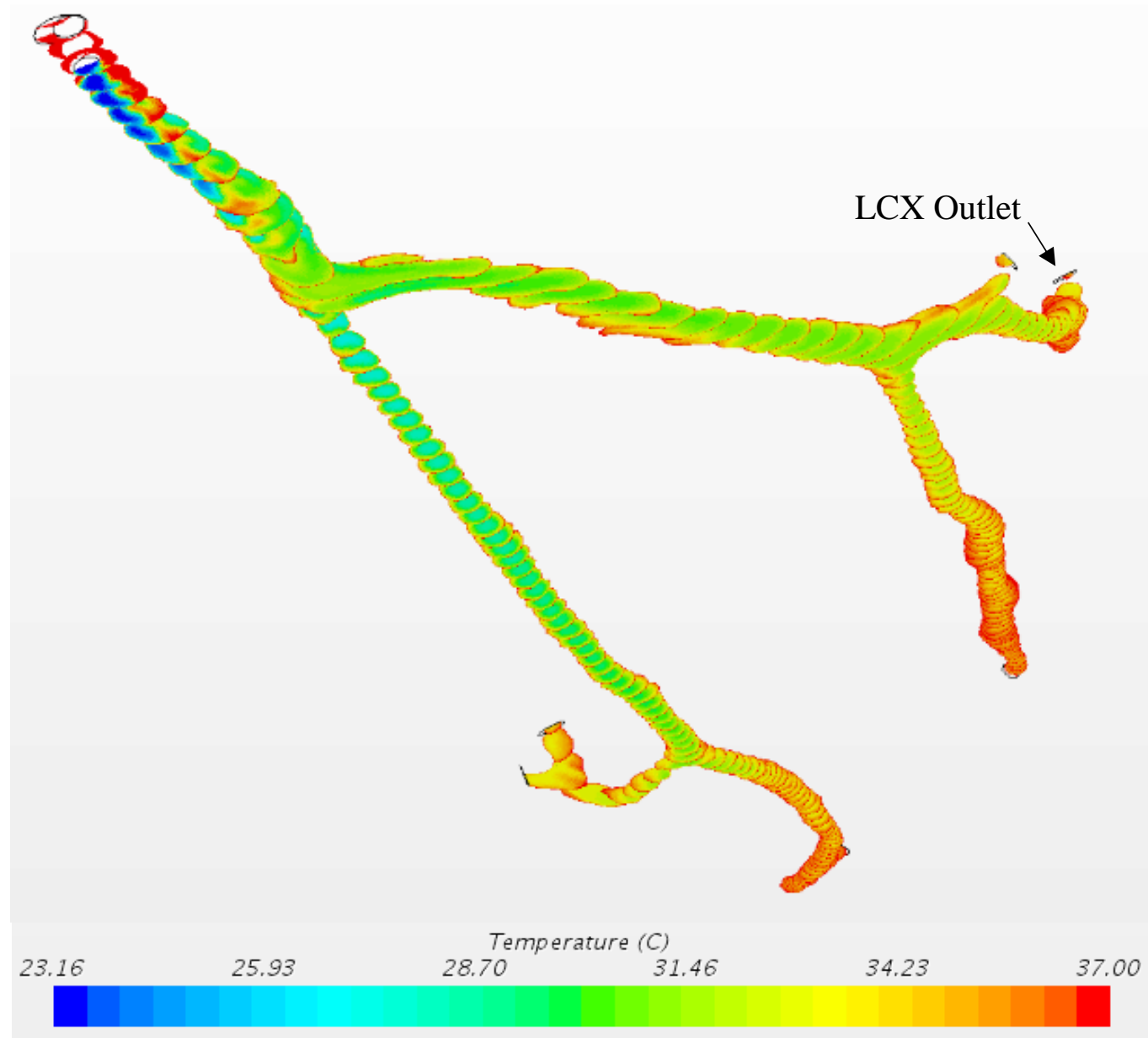

Figure 7.38. Structural-thermal relationship in HH102 LCX for $49.03 \mathrm{ml} / \mathrm{min}$ guide extension flow rate. Note: proximal guide extension inlet temperature is $23.16^{\circ} \mathrm{C}$, and LCX outlet temperature is $34.34^{\circ} \mathrm{C}$. 


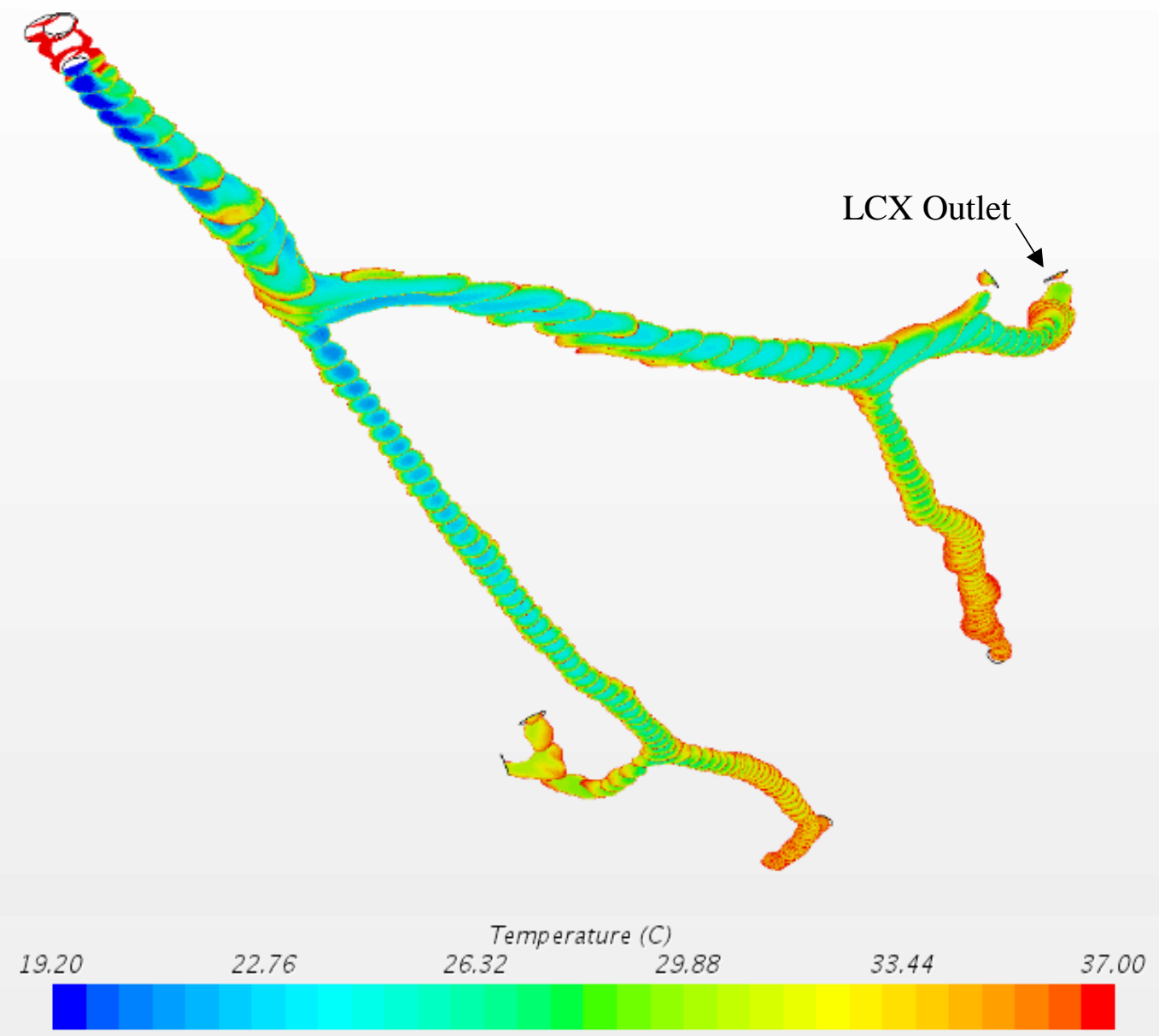

Figure 7.39. Structural-thermal relationship in HH102 LCX for $68.85 \mathrm{ml} / \mathrm{min}$ guide extension flow rate. Note: proximal guide extension inlet temperature is $19.2^{\circ} \mathrm{C}$, and LCX outlet temperature is $31.54^{\circ} \mathrm{C}$.

Intracoronary TH cooling techniques employed in our study resulted in predictions that were similar to the results of the following published works.

In a study performed by Otterspoor et al., chilled saline was delivered proximal to occluded arteries in the LAD using $10-30 \mathrm{~mL} / \mathrm{min}$ flow rates at $20^{\circ} \mathrm{C}[13]$. The goal of the study was to reach a target temperature of $33^{\circ} \mathrm{C}$ in the distal LAD using a 10 patient population. Temperature probes monitored the temperature of the saline during each operation while the occlusion was removed and reperfusion was induced (Fig. 7.40). 


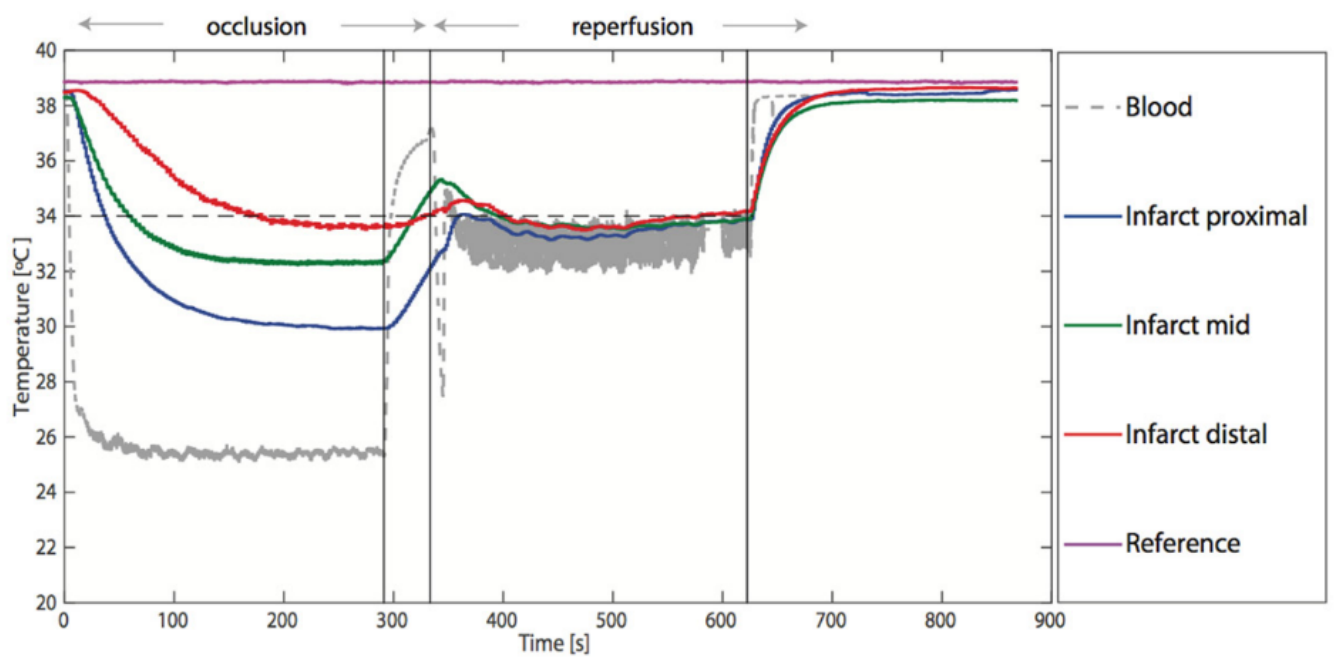

Figure 7.40. Temperature vs time plot for the proximal, mid, and distal infarct temperature probes during the infusion of chilled saline at $20^{\circ} \mathrm{C}$ in the coronary arteries. Proximal intracoronary temperatures reached $30^{\circ} \mathrm{C}$ during the occlusion phase and increased to $\sim 33^{\circ} \mathrm{C}$ for the duration of reperfusion prior to reaching body temperature at the end of the infusion.

A preceding study also analyzed a ten patient population but targeted their therapy towards patients with percutaneous coronary interventions (PCI) only, rather than those suffering from ST elevated myocardial infarction (STEMI). This study involved the use of TH during PCI with the goal of reducing intracoronary blood temperatures by $3.4^{\circ} \mathrm{C}$ $\left(33.6^{\circ} \mathrm{C}\right)$ at the occlusion site (Fig. 7.41) [97]. 


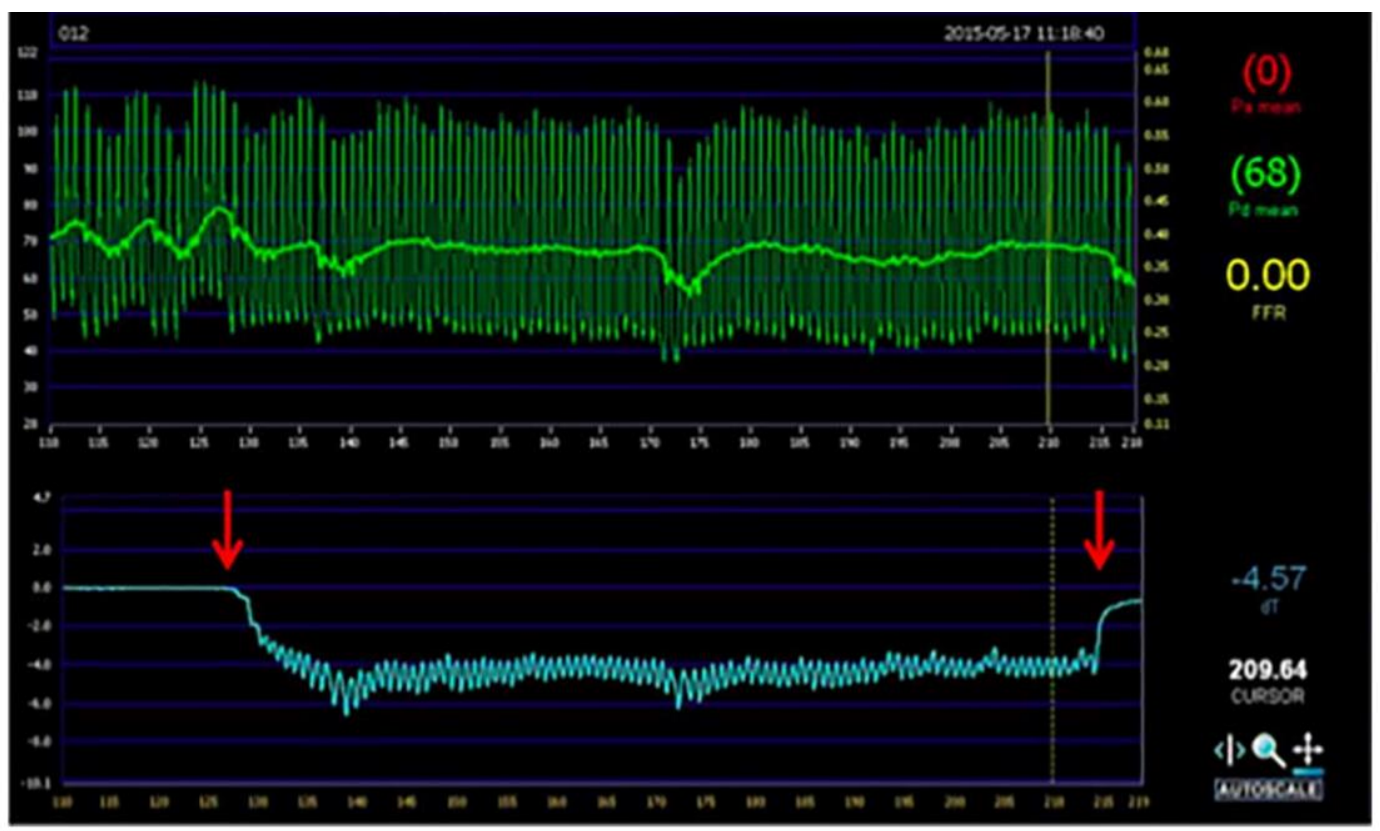

Figure 7.41. Temperature vs time display for intracoronary blood temperatures using TH therapy during PCI. Intracoronary blood temperatures were targeted consistently maintained around $33^{\circ} \mathrm{C}$ for the duration of the procedure. TH temperatures were maintained proximal to the occlusion in the coronary arteries for about 1 min and 15 seconds.

Each of the ten patients were infused with chilled saline proximal to an occlusion until their intracoronary blood temperatures reached $33^{\circ} \mathrm{C}$. Target temperatures of $33^{\circ} \mathrm{C}$ was consistently maintained for the duration of the procedure.

As observed in Fig. 7.40 and Fig. 7.41, intracoronary blood temperatures were reduced to $33^{\circ} \mathrm{C}$ locally in both the LAD and LCX arteries. Although these studies comprise of small patient populations, each study reported positive outcomes following the procedure with no signs of patient discomfort from shivering while reaching and maintaining target $\mathrm{TH}$ temperatures in minutes [13][97]. While these therapies observed the duration to reach and maintain target $\mathrm{TH}$ temperatures in the LAD and LCX arteries, the impact of reducing infarct and the relation between distal artery temperature with myocardial temperatures have yet to be explored. 
Despite the fact that localized TH targeted temperature management (TTM) is a relatively new therapy, Abbott Laboratories, a world renown biotechnology company, has recently initiated a randomized interventional clinical trial in Europe involving 200 patients with an estimated study duration of three years (January 1, 2019 to January 1, 2022) [146]. The goal of this study is to combine selective intracoronary hypothermia with primary percutaneous coronary interventions (PPCI) to reduce infarct size and permanent myocardial damage.

Similarly, our aim was to determine the impact of patient-specific coronary artery vascular structures on localized therapeutic hypothermia cooling. We chose six patientspecific LMCA representative heart (RH) models from the VHL and compared temperature distributions for a variety of flowrates in the LAD and LCX arteries. The structural thermal-fluid relationship was compared between each LAD and LCX simulation to determine cooling effectiveness levels. Based on their cooling effectiveness, we categorized each RH model as a preferred candidate or non-preferred candidate for localized heart cooling. The key takeaways and insights revealed from this work are discussed in Chapter 8. 


\section{Chapter 8}

\section{Conclusions and Future Work}

\section{Approaches and Recommendations for Cooling Hearts}

Patient-specific left anterior descending (LAD) and left circumflex (LCX) artery structures and their impact on cooling effectiveness have been quantified using the finite volume method. Surface area to volume ratios, relationships between mother and daughter branches, and effective depth of therapeutic hypothermia have been explored.

Preferred candidates for localized cooling consisted of heart structures that resulted in TH-ABT at the outlets. As illustrated in Fig. 7.32 from Chapter 7, a significant reduction in total network cooling effectiveness is evident when $\mathrm{SA}: \mathrm{V} \cdot$ total network length ratios exceed 700. Any value below this ratio resulted in total network cooling effectiveness levels greater than $97.63 \%$, while values above this ratio resulted in total network cooling effectiveness levels below $85.26 \%$. Therefore, preferred candidates had structural-thermal relationships linked to low values of SA:V ratios multiplied by total network length (Chapter 7 - Fig. 7.32).

High GuideLiner® infusate flow rates, specifically greater than and including $49.03 \mathrm{~mL} / \mathrm{min}$, were linked to TH-ABTs deep within the LAD and LCX mother vessels. Average cooling potential depth was slightly higher in the LAD mother vessel compared to each LCX mother vessel (Fig. 8.1). 


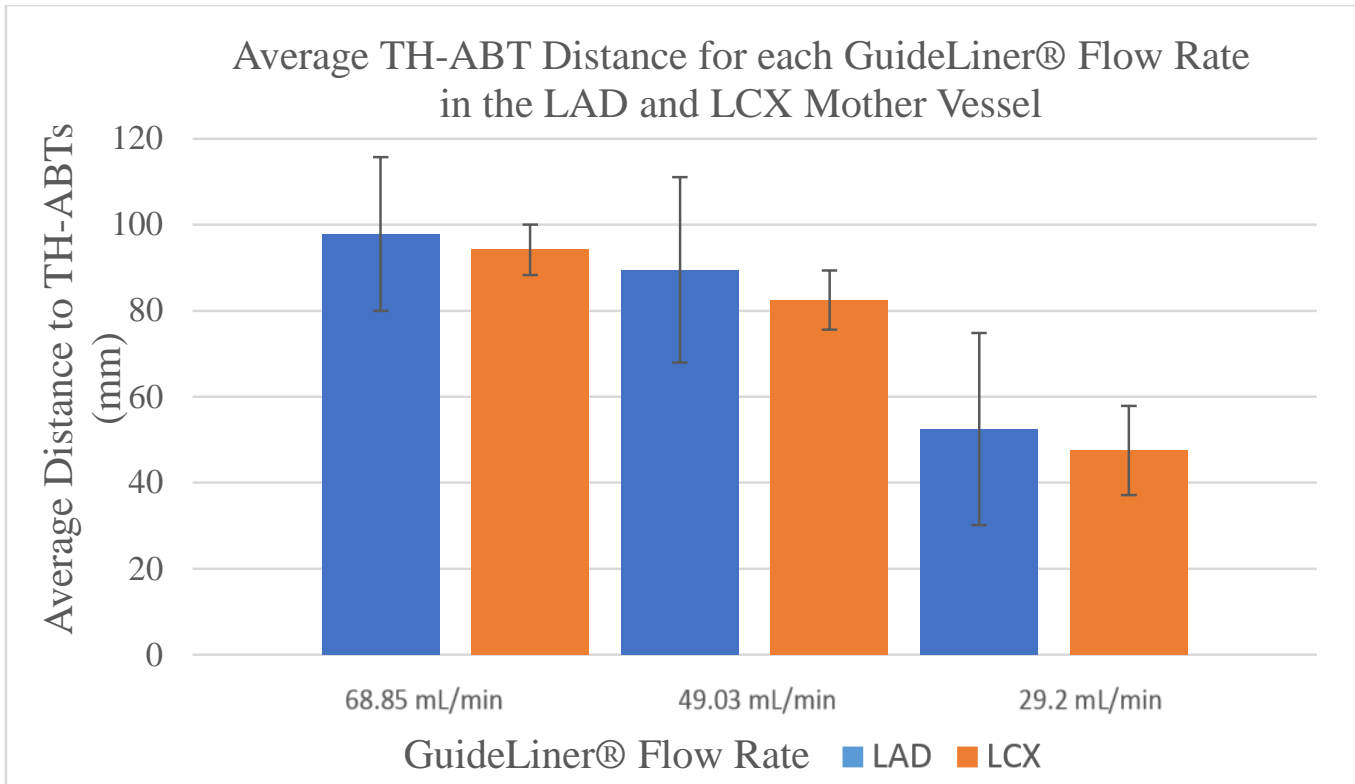

Figure 8.1. Average cooling potential depth in the LAD and LCX mother vessel. LAD average cooling potential was deeper in each mother vessel compared to the LCX. However, standard deviations were smaller in the LCX, especially for higher flow rates.

The standard deviation for each average TH-ABT depth potential was smaller with higher flow rates, and resulted in smaller deviations in the LCX arteries. Preferred heart cooling candidates were linked to GuideLiner® flow rates above $49.03 \mathrm{~mL} / \mathrm{min}$, which not only provided deeper cooling potential, but also provided a tighter standard deviation which improved accuracy for maintaining TH-ABTs.

A linear regression model was used to determine the relationship between input and output parameters from our CFD results. For this study, we analyzed the relationship between mother vessel CE (input) and $\eta_{T C}$ (output). These parameters were investigated for each GuideLiner® flow rate (Fig. 8.2) and a best fit linear regression model was used to determine how well our model fit the data. 

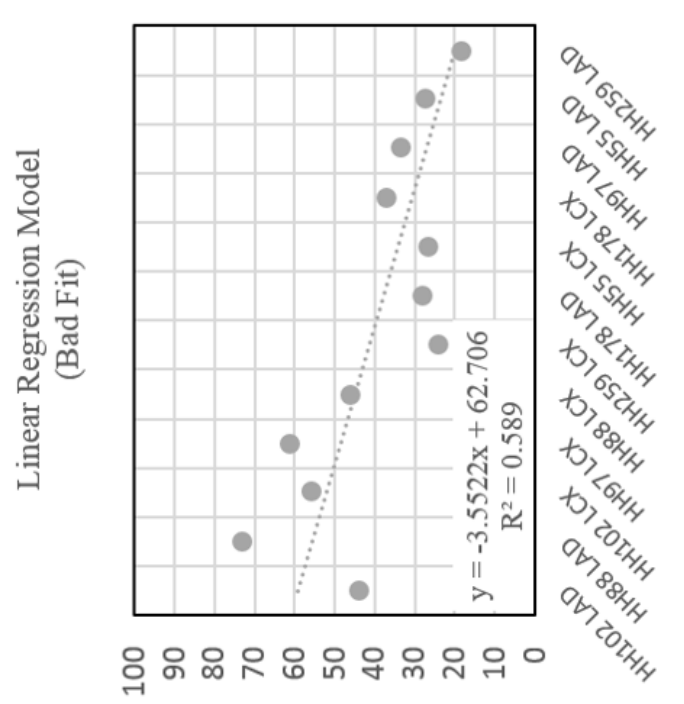

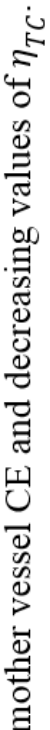
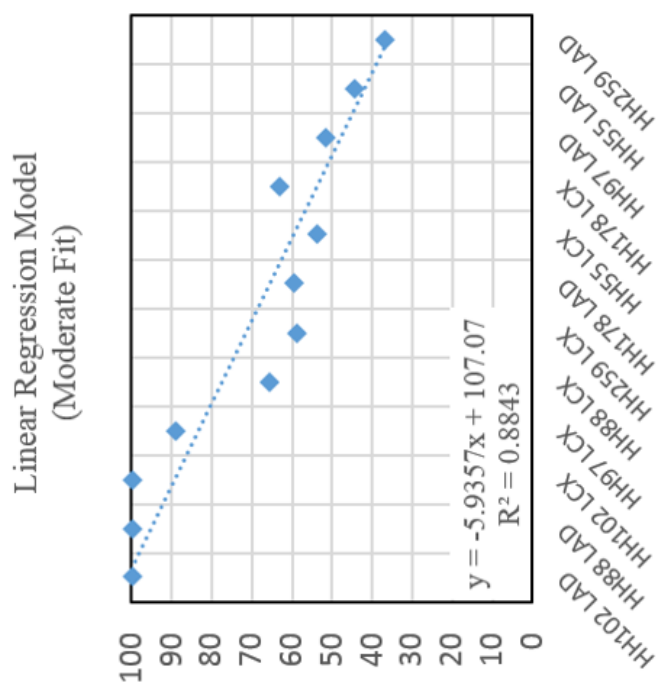

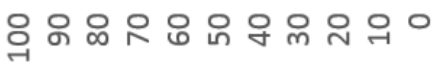

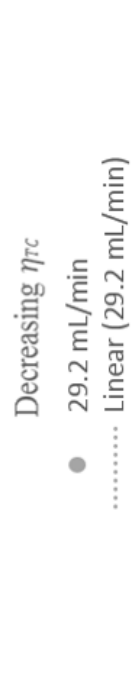

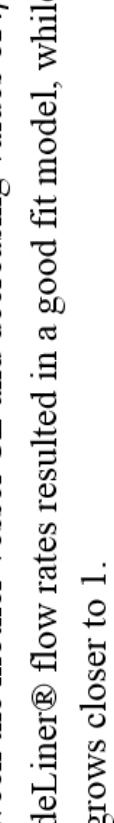

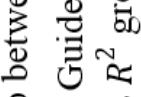

쿤 해

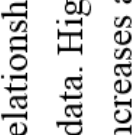
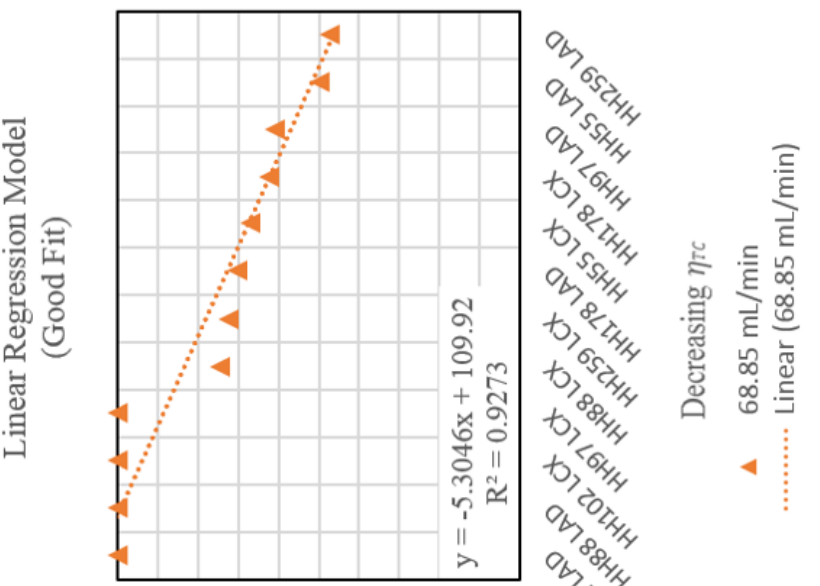

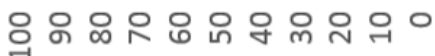
(\%) สว [ә⿻上丨

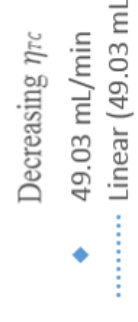

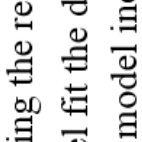

㐘㐘

苞

范

임

륧

它

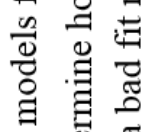

苛苛

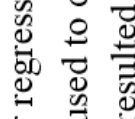

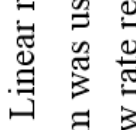

尚

o 2

运焉焉 
The linear regression model for each GuideLiner ${ }^{\circledR}$ flow rate resulted in the highest flow showing the most promise for providing an accurate model. High GuideLiner® flow rates $(68.85 \mathrm{~mL} / \mathrm{min})$ resulted in an $R^{2}$ value of 0.9273 , which statistically associated the percent variance between the dependent variable $\left(\eta_{T C}\right.$ for each $\mathrm{HH}$ model) and the independent variable (mother vessel CE). Medium GuideLiner® flow rates $(48.03 \mathrm{~mL} / \mathrm{min})$ resulted in having an $R^{2}$ value of 0.8843 , while the low flow rate ( $29.2 \mathrm{~mL} / \mathrm{min}$ ) resulting in an $R^{2}$ value of 0.589 , which is a statistically poor model. Therefore, the above linear regression models have demonstrated that GuideLiner® flow rates have a significant impact on $\eta_{T C}$ predictions.

Heart candidates that had fewer bifurcations stemming from the mother branch and fewer number of outlets in the artery network generally resulted in having better cooling outcomes, meaning TH-ABTs were able to travel deeper into the mother vessel. These results are illustrated by a linear fit trendline to the scatter plot data representing the number of diverting branches from the mother vessel (Fig. 8.3) and the number of outlets in the artery network (Fig. 8.4) with respect to decreasing values of $\eta_{T C}$. 
Diverting Branches from Mother Vessel vs Decreasing $\eta_{T C}$

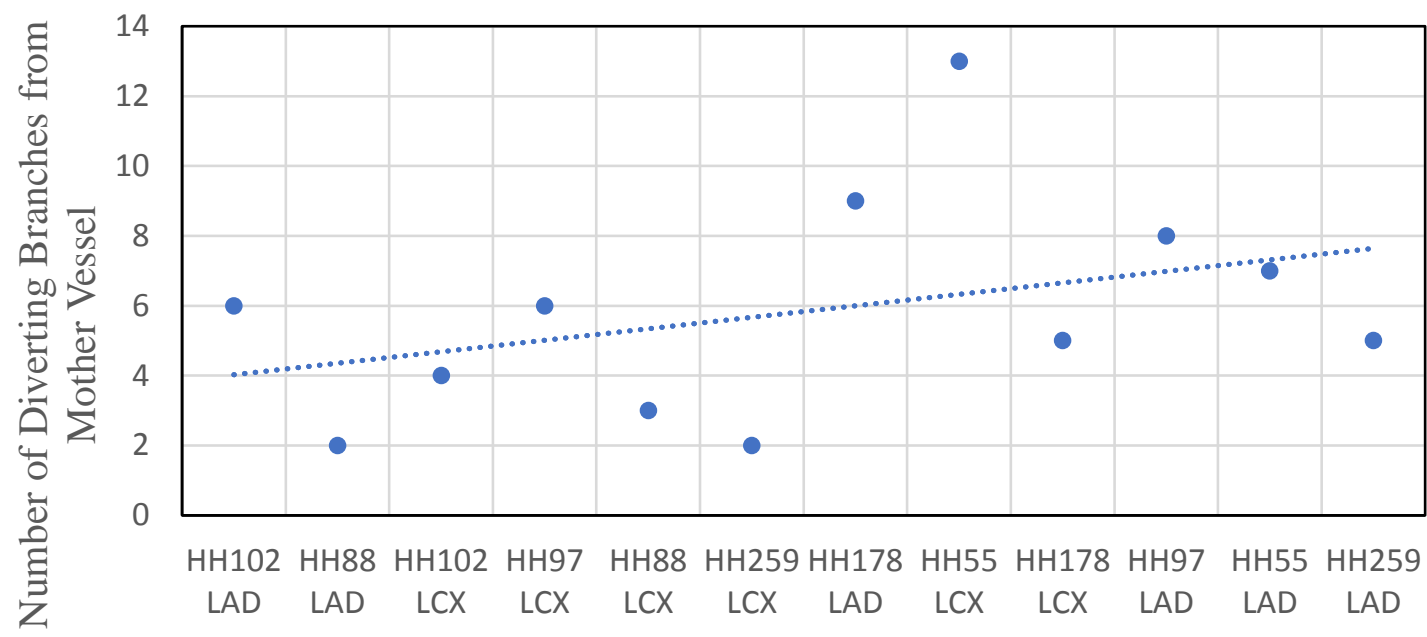

Decreasing $\eta_{T C}$

- Number of Diverting Branches

......... Linear (Number of Diverting Branches)

Figure 8.3. The relationship between the number of diverting branches from the mother vessel and decreasing total network cooling effectiveness $\left(\eta_{T C}\right)$ (from left to right). The linear fit trendline suggests that $\eta_{T C}$ is likely to decrease as the number of diverting branches from the mother vessel increase.

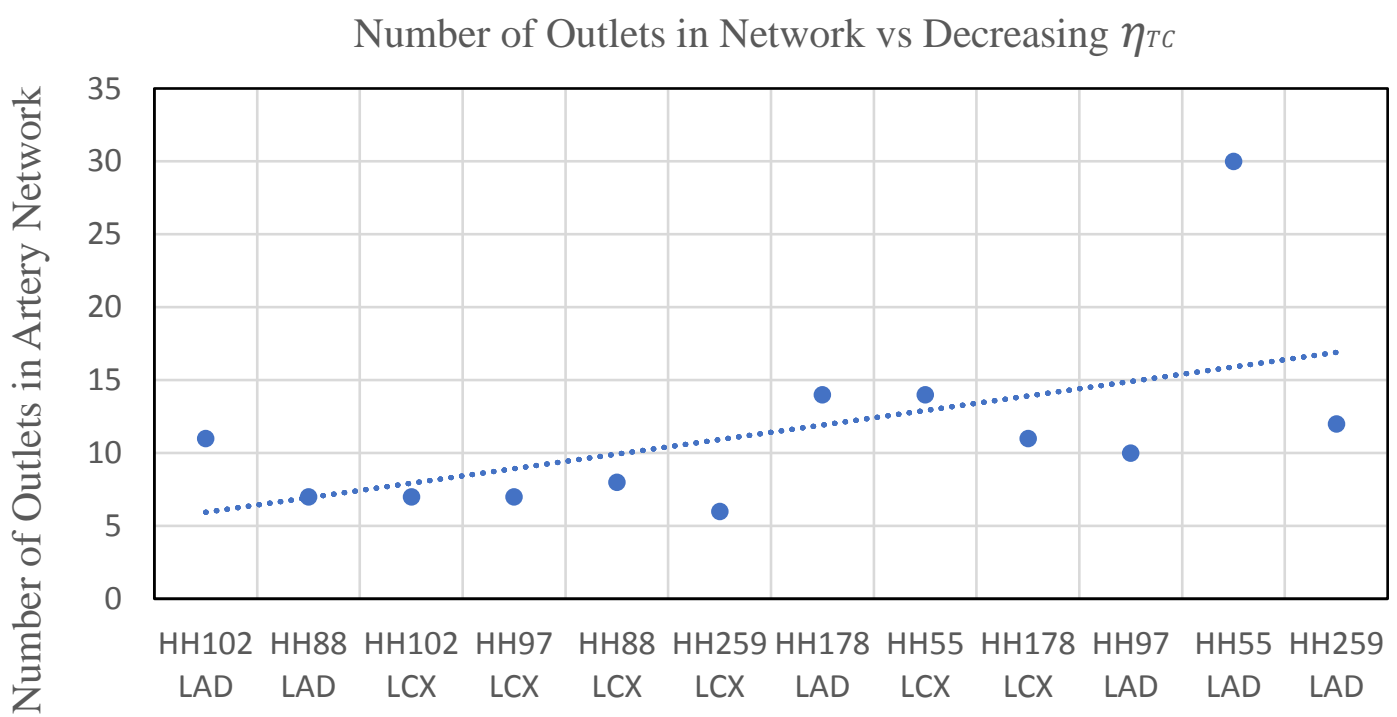

Decreasing $\eta_{T C}$

- Number of Outlets in Artery Network ....... Linear (Number of Outlets in Artery Network)

Figure 8.4. The relationship between the number of outlets in the LAD and LCX artery network and decreasing total network cooling effectiveness $\left(\eta_{T C}\right)$ (from left to right). The linear fit trendline suggests that $\eta_{T C}$ decreases as the number of outlets increase. 
Heart candidates that had an increasing amount of outlets in the LAD and LCX artery network tended to have poorer $\eta_{T C}$. The linear trendline model was used to determine a relationship between the number of diverging branches from the mother vessel and the number of outlets in the artery network for each candidate's $\eta_{T C}$. We recommend that the linear model only be considered as an optional parameter for determining optimal TH outcomes for each patient. Since a larger patient-specific population in needed to confirm the fidelity of this assumption, all LAD and LCX artery networks expressing these "features" are not necessarily indicative of preferred candidates for heart cooling.

To determine which methods promote effective cooling in the LAD and LCX arteries, the TH-ABT depth potential was analyzed. This method involved analyzing the ratio of $\mathrm{TH}-\mathrm{ABT}$ depth with respect to the length of the mother vessel and comparing those values to their corresponding flow rates in ascending order of SA:V ratios. A narrative of good and bad candidates for localized cooling in the LAD (Fig. 8.5) and LCX (Fig. 8.6) arteries is described with respect to effective TH-ABT levels. 


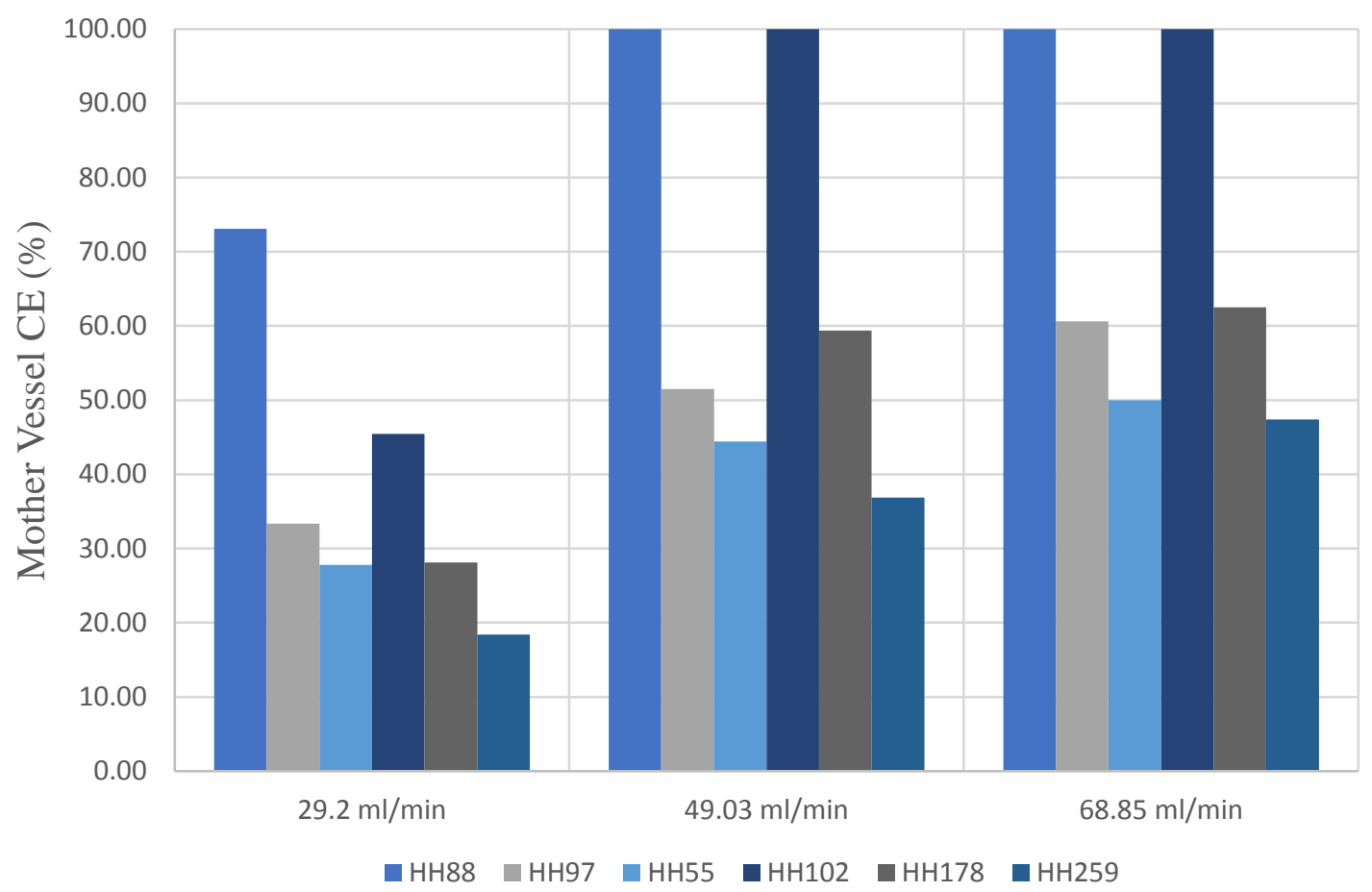

GuideLiner® Flow Rate

Figure 8.5. Percent of LAD pathway (mother vessel) experiencing TH-ABT. Increasing SA:V ratios are from left to right for each flow rate. HH88 and HH102 resulted in 100\% effective rates at 49.03 and $68.85 \mathrm{ml} / \mathrm{min}$ flow rates. Effective rates of $100 \%$ result in TH-ABT's $\left(<35^{\circ} \mathrm{C}\right)$ evident at the LAD outlets. 


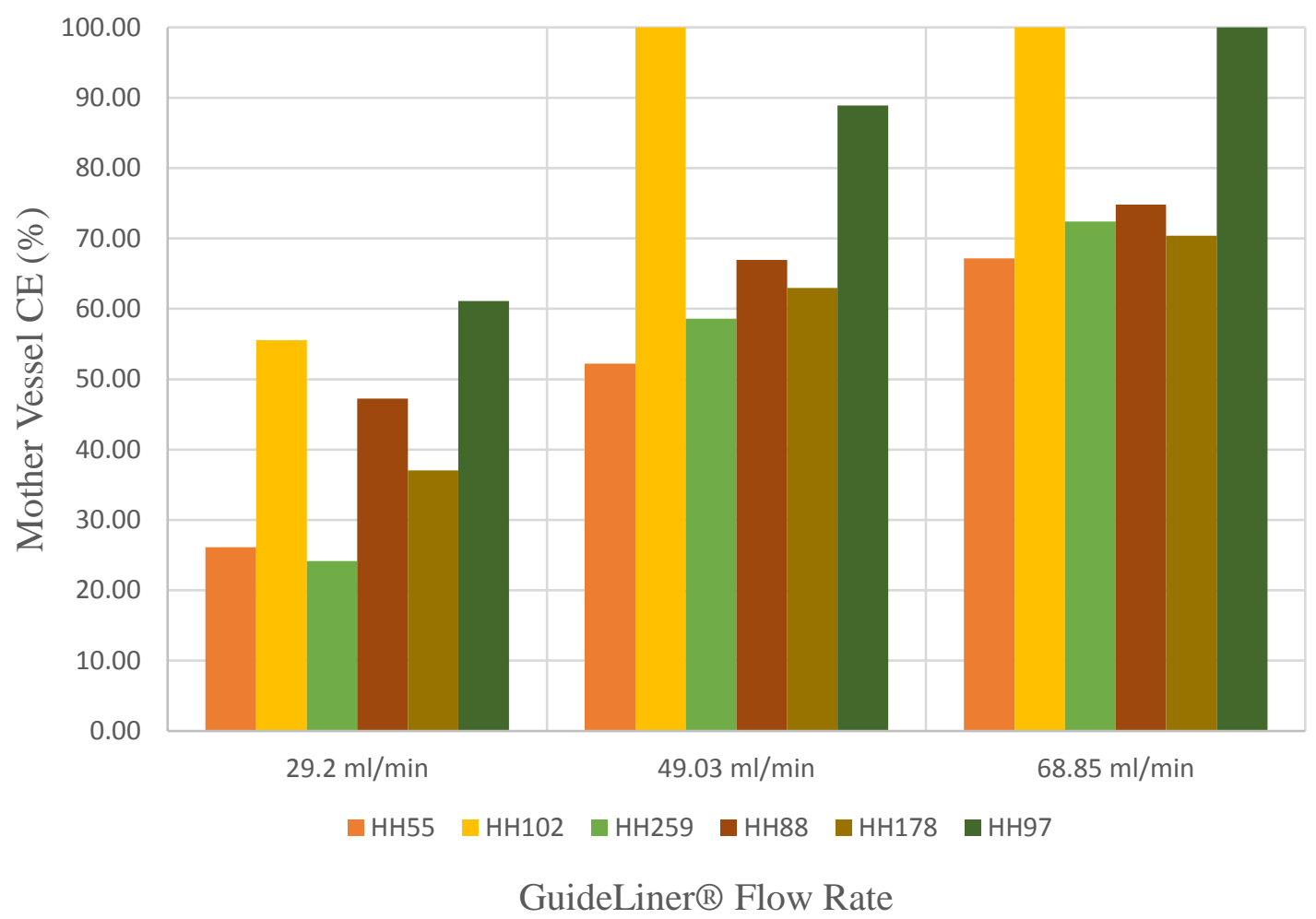

Figure 8.6. Percent of LCX pathway experiencing TH-ABT. Increasing SA:V ratios are from left to right for each flow rate. $\mathrm{HH} 102$ and $\mathrm{HH} 97$ resulted in 100\% effective rates at 49.03 and $68.85 \mathrm{ml} / \mathrm{min}$ flow rates in $\mathrm{HH} 102$ and $68.85 \mathrm{ml} / \mathrm{min}$ in HH97. Effective rates of $100 \%$ result in $\mathrm{TH}-\mathrm{ABT}$ 's $\left(<35^{\circ} \mathrm{C}\right)$ evident at the LCX outlets.

Preferred candidates for LAD and LCX heart cooling are represented by the bars that reached 100\% TH-ABT effective levels in Figures 8.5 and 8.6. This means that each bar reaching $100 \%$ effective levels had ABT's less than $35^{\circ} \mathrm{C}$ exiting the LAD and LCX mother branch outlets. Cooling effectiveness is determined by dividing the length of the LAD that reached $\mathrm{ABTs}<35^{\circ} \mathrm{C}$ divided by the total length of the LAD. For some patients, increasing the flow rate seemed to have little effect on adding cooling effectiveness in each LAD mother vessel. For the remaining patients, we can assume that $49.03 \mathrm{ml} / \mathrm{min}$ GuideLiner® flow rate is sufficient to use in TH intracoronary procedures in lieu of $68.85 \mathrm{ml} / \mathrm{min}$. The $\mathrm{SA}: \mathrm{V}$ ratios are illustrated from left to right in increasing 
order (Fig. 8.5), which resulted in no identifiable trends that link cooling effectiveness to increased values of SA:V for the entire LAD network.

A slight trend between high SA:V ratios in most of the patient-specific LCX models is apparent compared to low SA:V ratios (Fig. 8.6). Cooling effectiveness in the LCX appeared to trend differently than the LAD data where increased SA:V ratios in the LCX tended to lead towards greater cooling effectiveness. Contrarily, the LAD resulted in no identifiable trends between SA:V ratios and cooling effectiveness. GuideLiner® flow rates above and including $49.03 \mathrm{ml} / \mathrm{min}$ were $33.3 \%$ effective at delivering TH-ABT at the distal LAD outlets, and $25 \%$ effective at the LCX outlets.

The remaining $66.7 \%$ of LAD candidates that experienced proximal flow rates greater than $49.03 \mathrm{ml} / \mathrm{min}$ were considered bad candidates for heart cooling since their TH-ABT's only traveled $\leq 60 \%$ into the mother branches, with the exception of one that travelled $73.08 \%$ into the mother branch (Fig. 8.5). Additionally, 75\% of LCX candidates that experienced proximal flow rates greater than $49.03 \mathrm{ml} / \mathrm{min}$ were considered bad candidates for heart cooling since their TH-ABT's only traveled $\leq 74.8 \%$ into the mother branches, with the exception of one that travelled $88.9 \%$ into the mother branch (Fig. 8.6).

Decreasing values of $\eta_{T C}$ were compared to the ABT at each LAD and LCX artery mother vessel outlet. ABT data for all three GuideLiner® flow rates were plotted vs decreasing $\eta_{T C}$ and the seven preferred heart cooling candidates were isolated using a dashed box (Fig. 8.7). 
ABT at Mother Vessel Outlet for each GuideLiner® Flow Rate

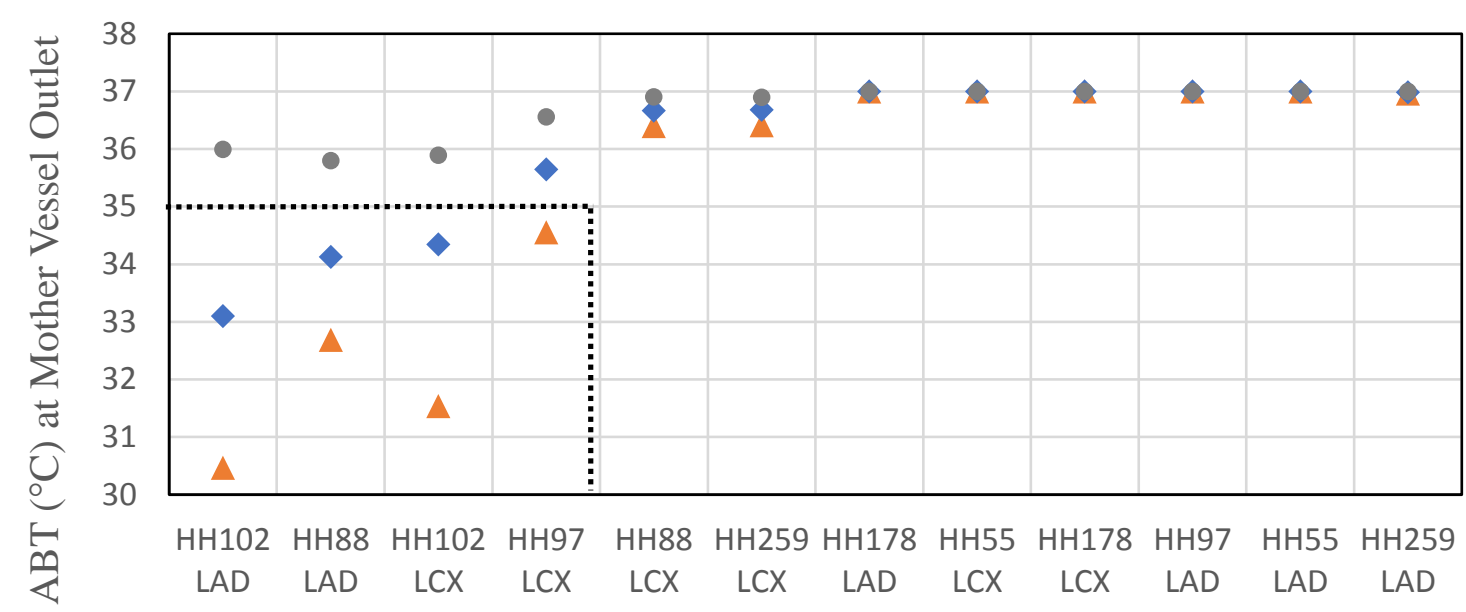

Decreasing $\eta_{T C}$

$$
\Delta 68.85 \mathrm{~mL} / \mathrm{min} \quad \bullet 49.03 \mathrm{~mL} / \mathrm{min} \quad \bullet 29.2 \mathrm{~mL} / \mathrm{min}
$$

Figure 8.7. Mother vessel outlet ABT data plots for each GuideLiner® flow rate vs decreasing $\eta_{T C}$ values. Seven recommended heart cooling candidates are isolated in the dashed box which are segregated by TH-ABTs $<35^{\circ} \mathrm{C}$ (horizontal line) and $\eta_{T C}>97.63 \%$ (vertical line).

TH-ABTs were evident in the remaining seven out of the total thirty-six CFD simulations at the distal LAD and LCX posterolateral outlets for $49.03 \mathrm{ml} / \mathrm{min}$ and 68.85 $\mathrm{ml} / \mathrm{min}$ flow rates. The associated distance for each ABT value is summarized in Table 8.1.

Table 8.1

GuideLiner ${ }^{\circledR}$ flow rates associated with TH-ABT's leaving the LAD and LCX outlets.

\begin{tabular}{lcccc}
\hline \multirow{2}{*}{ Location } & \multicolumn{2}{c}{ Flowrate @ } & $\mathbf{4 9 . 0 3 ~ \mathbf { ~ m l } / \mathbf { m i n }}$ & \multicolumn{2}{c}{ Flowrate @ } & $\mathbf{6 8 . 8 5} \mathbf{~ m l} / \mathbf{m i n}$ \\
\cline { 2 - 5 } & $\begin{array}{c}\text { Distance to } \\
\text { Outlet }(\mathbf{m m})\end{array}$ & $\begin{array}{c}\text { ABT at } \\
\text { Outlet }\left({ }^{\circ} \mathbf{C}\right)\end{array}$ & $\begin{array}{c}\text { Distance to } \\
\text { Outlet }(\mathbf{m m})\end{array}$ & $\begin{array}{c}\text { ABT } @ \\
\text { Outlet }\left({ }^{\circ} \mathbf{C}\right)\end{array}$ \\
HH88 LAD & 130 & 34.13 & 130 & 32.69 \\
HH97 LCX & --- & --- & 90 & 34.55 \\
HH102 LAD & 80 & 33.1 & 80 & 30.47 \\
HH102 LCX & 90 & 34.34 & 90 & 31.54 \\
\hline
\end{tabular}


TH-ABT in the LAD and LCX mother vessels varied in temperature at the outlet for each $\mathrm{HH}$ model and GuideLiner® flow rate. As shown previously, higher flow rates tended to yield cooler ABTs along the artery pathway. However, Table 8.1 shows that not all patients experiencing $68.85 \mathrm{~mL} / \mathrm{min}$ GuideLiner® flow rates revealed lower ABTs compared to $49.02 \mathrm{~mL} / \mathrm{min}$ GuideLiner ${ }^{\circledR}$ low rates. While each patient-specific coronary artery network is unique to the number of branches, outlets, and artery dimensions it has, the critical factor to consider is that ABTs vary in each patient's LAD and LCX artery regardless of GuideLiner® flow rate.

Summary. In summary, three distinguishable methods were obtained from this work and used to distinguish between good and bad candidates for localized heart cooling (Table 8.2).

Table 8.2

Good and bad heart cooling candidates as recommended assuming $68.85 \mathrm{~mL} / \mathrm{min}$ proximal GuideLiner ${ }^{\circledR}$ flow rate.

\begin{tabular}{|c|c|c|c|}
\hline $\begin{array}{l}\text { Insight } \\
\text { Number }\end{array}$ & \multicolumn{3}{|c|}{ Good/Bad Candidate Evaluation for Recommended $\eta_{\text {TC }}$} \\
\hline \multirow[t]{3}{*}{1} & $\begin{array}{l}\text { Number of diverting branches from } \\
\text { mother vessel (Fig. 8.3) }\end{array}$ & $\begin{array}{l}\text { Recommended } \\
\text { cut-off value }\end{array}$ & Avg. / ST Dev. \\
\hline & Good Candidate & $\leq 6$ & $4.5+/-1.9$ \\
\hline & Bad Candidate & $\geq 7$ & $6.5+/-3.5$ \\
\hline \multirow[t]{3}{*}{2} & $\begin{array}{l}\text { Number of outlets in artery } \\
\text { network (Fig. 8.4) }\end{array}$ & & \\
\hline & Good Candidate & $\leq 11$ & $6.4+/-2.0$ \\
\hline & Bad Candidate & $\geq 12$ & $13.1+/-7.4$ \\
\hline \multirow[t]{3}{*}{3} & $\begin{array}{l}\text { SA:V • Total Network Length } \\
\text { (Fig. 7.25) }\end{array}$ & & \\
\hline & Good Candidate & $\leq 700$ & $539.2+/-108.8$ \\
\hline & Bad Candidate & $\geq 701$ & $893+/-210$ \\
\hline
\end{tabular}

Note. Recommended cut-off value for good candidates represents the max value where $\eta_{T C} \geq 97.63 \%$. The cut-off value for bad candidates represents the lowest value where $\eta_{T C} \leq 85.26 \%$. Values 1 and 2 are estimated values determined using linear regression models and should only be considered in combination with insight number 3 . 
While these insights help to provide recommended guidelines for determining preferred candidates for heart cooling, a few exceptions were identified in bad candidate hearts where good candidate characteristics were evident. Patient HH259 is a good example of a bad heart cooling candidate even though it contained LAD and LCX branches stemming from the mother vessel that fell within preferred candidate ranges (Table 8.3), and had the largest SA:V ratio out of all six LAD representative heart (RH) models.

Table 8.3

Good and bad heart cooling candidates with their corresponding number of outlets and vessel branches stemming from the mother branch.

\begin{tabular}{|l|c|c|c|c|}
\hline & $\begin{array}{c}\text { LAD } M_{v} \\
\text { Diverting Branches }\end{array}$ & $\begin{array}{c}\text { LCX } M_{v} \\
\text { Diverting Branches }\end{array}$ & $\begin{array}{c}\text { LAD } T_{n} \\
\text { Outlets }\end{array}$ & $\begin{array}{c}\text { LCX } T_{n} \\
\text { Outlets }\end{array}$ \\
\hline HH55 & 7 & 13 & 14 & 30 \\
\hline HH88 & 2 & 3 & 7 & 8 \\
\hline HH97 & 8 & 6 & 10 & 7 \\
\hline HH102 & 6 & 4 & 11 & 7 \\
\hline HH178 & 9 & 5 & 14 & 11 \\
\hline HH259 & 5 & 2 & 12 & 6 \\
\hline
\end{tabular}

Note. Mother vessel $=M_{v}$ and total network $=T_{n}$. Preferred heart cooling candidates are highlighted. Results represent inlet flow rates greater than and including $49.03 \mathrm{ml} / \mathrm{min}$.

A combination of the three methods described in Table 8.2 for determining which candidates are suitable for localized intracoronary heart cooling involved determining the number of branches stemming from the mother branch of interest, the number of outlets in the LAD and LCX artery networks, and the surface area to volume ratio multiplied by the total network length for each patient-specific LAD and LCX artery. Implementing these three methods, as well as employing the appropriate proximal GuideLiner ${ }^{\circledR}$ flow rates prior to a localized intracoronary heart cooling procedure will help to provide insight on the potential outcome of cooling effectiveness. The computational work 
described herein provides a foundation to guide cardiovascular interventionist's use of therapeutic hypothermia to optimize cooling performance and patient outcomes.

\section{Future work}

Geometry. Future work for this study should be focused on attaining additional heart models from the Visible Heart Lab (VHL) website and additional resources where higher DICOM image resolutions are available. Since the vasculature is not rigid, future work should also explore fluid-structure interaction.

Additional heart models. Implementing additional heart models will introduce more anatomical variations which could help improve the accuracy of our predicted $\eta_{T C}$ to $\mathrm{SA}: \mathrm{V} \cdot$ total network length ratios in each mother vessel. Executing a large range of patient-specific heart models will broaden the scope of this work and will introduce an extensive collection of patient-specific vascular networks for determining their impact on localized TH cooling.

Physics. The physics solvers employed in the STAR-CCM+ simulations were constrained to steady state laminar flow conditions. Each of the mass flow inlet conditions were performed using constant flow rates that exhibited no pulsatile affects. Future studies should utilize a pulsatile field function in STAR-CCM+ for both the blood and GuideLiner® inlet conditions. This would produce temperature distributions that would accurately resemble pulsatile blood flow conditions in the coronary arteries.

Non-mixed blood properties should be used at the start of each simulation and normal saline at $0.9 \% \mathrm{NaCl}$ should be used for the GuideLiner® flow instead of distilled water. Blood flow behavior would also be improved if unsteady state conditions are applied to each model so that time dependent hemodynamic conditions and hematocrit levels are considered [4][75][106][123]. 
Extending arterial blood temperature predictions towards tissue predictions.

Vessel wall thickness in future studies should be considered and replicated to extend arterial blood temperature predictions towards tissue predictions. Each of the patientspecific LAD and LCX models had fixed boundary conditions assuming a rigid no-slip wall boundary, which should be improved to include wall thicknesses to ultimately provide a more accurate representation of the temperature distributions in each artery network.

Validation. Since CFD modeling provides an alternative to experimental development, CFD simulations must always be scrutinized and compared with experimental results and published data to validate each model's credibility [134].

In vivo validation of cooling effectiveness and myocardial salvage. To validate our patient-specific representative heart $(\mathrm{RH})$ models, future efforts should focus on patient-specific $3 \mathrm{D}$ printed replicas that can be used in in vitro mock loop systems to validate the predicted CFD average bulk temperatures.

Furthermore, in vivo validations could help validate our cooling effectiveness levels and methods used to determine good candidates for localized TH cooling. Localized TH cooling studies performed in swine are also excellent models that should be used to explore additional cooling methods and outcomes [13].

The methods described in this thesis work are used to identify ideal candidates for localized heart cooling and can be improved and applied in clinical settings where patients suffering from SA and STEMI could be recommended as ideal candidates for localized intracoronary cooling to ultimately enhance myocardial salvage [99][96]. 


\section{References}

[1] Jonathan D. Kibble, PhD, Colby R. Halsey, Medical Physiology: The Big Picture. 2009.

[2] W. Achilles J. Pappano, Cardiovascular Physiology. Elsevier, 2013.

[3] Wapcaplet, "Diagram of the human heart," Wikimedia Commons, 2005. .

[4] B. Dfu and B. D. Jo, "Hemodynamics inside a human left coronary artery," vol. 5, no. 7, pp. 910-914, 2015.

[5] V. F. Richard A. Walsh, James C. Fang, Hurst's The Heart. McGraw Hill Medical, 2013.

[6] A. D. Villa et al., "Coronary artery anomalies overview: The normal and the abnormal," World J. Radiol., vol. 8, no. 6, pp. 537-556, 2016.

[7] P. Territories et al., "Aligning Coronary Anatomy and Myocardial Perfusion Territories: An Algorithm for the CORE320 Multicenter Study," Am. Hear. Assoc., pp. 587-595, 2012.

[8] C. Smuclovisky, “Coronary Artery CTA,” in Springer, McGraw-Hill, 2010.

[9] W. Wang, X. Zhao, B. Mao, Y. Liu, and Z. Zhao, "A method to personalize the lumped parameter model of coronary artery," no. 100.

[10] H. J. Kim, I. E. Vignon-Clementel, J. S. Coogan, C. A. Figueroa, K. E. Jansen, and C. A. Taylor, "Patient-specific modeling of blood flow and pressure in human coronary arteries," Annals of Biomedical Engineering, vol. 38, no. 10. pp. 31953209, 2010.

[11] H. Wieneke, "Determinants of coronary blood flow in humans: quantification by intracoronary Doppler and ultrasound," J. Appl. Physiol., vol. 98, no. 3, pp. 10761082, 2004.

[12] K. Johnson, P. Sharma, and J. Oshinski, "Coronary Artery Flow Measurement Using Navigator Echo Gated Phase Contrast Magnetic Resonance Velocity Mapping at 3.0 Tesla," vol. 41, no. 3, pp. 595-602, 2008.

[13] L. C. Otterspoor, Intracoronary hypothermia. Technische Universiteit Eindhoven, 2018.

[14] W. Aarnoudse et al., "Direct Volumetric Blood Flow Measurement in Coronary Arteries by Thermodilution," J. Am. Coll. Cardiol., vol. 50, no. 24, pp. 2294-2304, 2007. 
[15] H. Zafar, F. Sharif, and M. J. Leahy, "Measurement of the blood flow rate and velocity in coronary artery stenosis using intracoronary frequency domain optical coherence tomography: Validation against fractional flow reserve," IJC Hear. Vasc., vol. 5, pp. 68-71, 2014.

[16] H. V. Anderson, M. J. Stokes, M. Leon, S. A. Abu-halawa, Y. Stuart, and R. L. Kirkeeide, "Coronary Artery Flow Velocity Is Related To Lumen Area and Regional Left Ventricular Mass," Circulation, pp. 48-55, 2000.

[17] B. S. Bruce Koeppen, Berne \& Levy Physiology. 2017.

[18] A. Rao, Y. Pimpalwar, N. Yadu, and R. K. Yadav, "A study of coronary artery variants and anomalies observed at a tertiary care armed forces hospital using 64slice MDCT," Indian Heart J., vol. 69, pp. 81-86, 2017.

[19] S. Lakshmiprabha, K. H. Afroze, P. Ramesh, K. R. Asha, C. Shivaleela, and D. Anupama, "Variations in the anatomical and branching pattern of the left coronary artery : a cadaveric study," Int. J. Res. Med. Sci., vol. 6, no. 4, pp. 1235-1240, 2018 .

[20] C. Altin et al., "Coronary anatomy, anatomic variations and anomalies : a retrospective coronary angiography study," Singapore Med. J., vol. 56, no. 7, pp. $339-345,2015$.

[21] D. R. Anand M. Rahalkar, "Pictorial essay: Coronary artery variants and anomalies," Indian J. Radiol. Imaging, 2009.

[22] P. Libby, "Mechanisms of Acute Coronary Syndromes and Their Implications for Therapy," N. Engl. J. Med., vol. 368, no. 21, pp. 2004-2013, 2013.

[23] K. Thygesen, J. S. Alpert, A. S. Jaffe, M. L. Simoons, B. R. Chaitman, and H. D. White, "Third universal definition of myocardial infarction," Nat. Rev. Cardiol., vol. 9, no. 11, pp. 620-633, 2012.

[24] B. Ibáñez, G. Heusch, M. Ovize, and F. Van De Werf, "Evolving therapies for myocardial ischemia/reperfusion injury," J. Am. Coll. Cardiol., vol. 65, no. 14, pp. 1454-1471, 2015.

[25] J. L. Anderson and D. A. Morrow, “Acute Myocardial Infarction," N. Engl. J. Med., vol. 376, no. 21, pp. 2053-2064, 2017.

[26] E. J. Benjamin et al., Heart Disease and Stroke Statistics-2018 Update: A Report From the American Heart Association. 2018.

[27] M. PRINZMETAL, "The collateral circulation of the human heart.," Mod. Concepts Cardiovasc. Dis., vol. 15, no. 10, p. [1], 2013. 
[28] T. Cung et al., "Cyclosporine before PCI in Patients with Acute Myocardial Infarction," N. Engl. J. Med., vol. 373, no. 11, pp. 1021-1031, 2015.

[29] M. Esposito, Y. Bader, R. Pedicini, C. Breton, A. Mullin, and N. K. Kapur, "The role of acute circulatory support in ST-segment elevation myocardial infarction complicated by cardiogenic shock," Indian Heart J., vol. 69, no. 5, pp. 668-674, 2017.

[30] C. Rihal et al., "2015 SCAI/ACC/HFSA/STS Clinical Expert Consensus Statement on the Use of Percutaneous Mechanical Circulatory Support Devices in Cardiovascular Care,” J. Am. Coll. Cardiol., vol. 65, no. 19, pp. e7-e26, 2015.

[31] J. Ezekowitz, P. Kaul, J. A. Bakal, P. W. Armstrong, R. C. Welsh, and F. A. McAlister, "Declining In-Hospital Mortality and Increasing Heart Failure Incidence in Elderly Patients With First Myocardial Infarction," J. Am. Coll. Cardiol., vol. 53, no. 1, pp. 13-20, 2009.

[32] G. Fröhlich, P. Meier, S. K. White, D. M. Yellon, and D. J. Hausenloy, "Myocardial reperfusion injury: Looking beyond primary PCI," Eur. Heart J., vol. 34, no. 23, pp. 1714-1724, 2013.

[33] D. Yellon and D. J. Hausenloy, "Myocardial reperfusion injury," N. Engl. J. Med., vol. 357, pp. 1121-1135, 2007.

[34] C. Fordyce CB, Gersh BJ, "Novel therapeutics in myocardial infarction: targeting microvascular dysfunction and reperfusion injury," Trends Pharmacol Sci, 2015.

[35] Kapur NK and Karas RH, "A new shield from the double-edged sword of reperfusion in STEMI,” Eur Hear., vol. 36, pp. 3058-60, 2015.

[36] D. J. Hausenloy et al., "Targeting reperfusion injury in patients with ST-segment elevation myocardial infarction: Trials and tribulations," Eur. Heart J., vol. 38, no. 13, pp. 935-941d, 2017.

[37] P. S. Chan, R. A. Berg, Y. Tang, L. H. Curtis, and J. A. Spertus, "Association between therapeutic hypothermia and survival after in-hospital cardiac arrest," JAMA - J. Am. Med. Assoc., vol. 316, no. 13, pp. 1375-1382, 2016.

[38] M. Luscombe and J. C. Andrzejowski, "Clinical applications of induced hypothermia," Contin. Educ. Anaesthesia, Crit. Care Pain, vol. 6, no. 1, pp. 2327, 2006.

[39] P. D. Shlee S. Song, "Overview of Therapeutic Hypothermia," Curr Treat Options Neurol, vol. 14, no. 6, pp. 541-548, 2012.

[40] R. Lakshmanan, F. Sadaka, and A. Palagiri, "Therapeutic Hypothermia: Adverse Events, Recognition, Prevention and Treatment Strategies," Ther. Hypothermia Brain Inj., pp. 3-20, 2013. 
[41] S. P. Jones et al., "The NHLBI-Sponsored Consortium for preclinicAl assESsment of cARdioprotective Therapies (CAESAR): A new paradigm for rigorous, accurate, and reproducible evaluation of putative infarct-sparing interventions in mice, rabbits, and pigs," Circ. Res., vol. 116, no. 4, pp. 572-586, 2015.

[42] R. Fernández-jiménez and B. Ibanez, "One Step Beyond in the Construction of a Translational Bridge,” pp. 554-557.

[43] W. Desmet et al., "High-dose intracoronary adenosine for myocardial salvage in patients with acute ST-segment elevation myocardial infarction," Eur. Heart J., vol. 32, no. 7, pp. 867-877, 2011.

[44] S. W. Gruntzig AR, Senning A, "Nonoperative dilatation of coronary-artery stenosis: percutaneous transluminal coronary angioplasty," N. Engl. J. Med., vol. 301, pp. 61-68, 1979.

[45] W. Maier, S. Windecker, E. Boersma, and B. Meier, "Evolution of percutaneous transluminal coronary angioplasty in Europe from 1992-1996," Eur. Heart J., vol. 22, no. 18, pp. 1733-1740, 2001.

[46] R. H. Puel J, Joffre F, "Endo-protheses coronariennes auto-expansives dans la prevention des restenoses apres angioplastie transluminale," Coeur, arch mal, vol. 8, pp. 1311-1312, 1987.

[47] K. A. Schomig A, Neumann FJ, "A randomized comparison of antiplatelet and anticoagulant therapy after the placement of coronary-artery stents," N. Engl. J. Med., vol. 334, pp. 1084-1089, 1996.

[48] B. M. Zijlstra F, Hoorntje JC, "Long-term benefit of primary angioplasty as compared with thrombolytic therapy for acute myocardial infarction," N. Engl. J. Med., vol. 341, pp. 1413-1419, 1999.

[49] Wijeysundera HC, Machado M, Farahati F, Wang X, Witteman W, van der Velde $\mathrm{G}$, "Association of temporal trends in risk factors and treatment uptake with coronary heart disease mortality, 1994-2005," JAMA - J. Am. Med. Assoc., vol. 303, no. 18, pp. 1841-1847, 2010.

[50] S. S. Afana M1, Brinjikji W, Cloft H, "Hospitalization costs for acute myocardial infarction patients treated with percutaneous coronary intervention in the United States are substantially higher than Medicare payments.," Clin. Cardiol., vol. 38, no. 1, pp. 13-19, 2015.

[51] Rasha Al-Lamee et al., "Percutaneous coronary intervention in stable angina (ORBITA): a double-blind, randomised controlled trial," Lancet, vol. 391, no. 10115, pp. 31-40, 2018. 
[52] P. T. O’Gara et al., “2013 ACCF/AHA guideline for the management of stelevation myocardial infarction: A report of the American college of cardiology foundation/american heart association task force on practice guidelines," J. Am. Coll. Cardiol., vol. 61, no. 4, pp. 78-140, 2013.

[53] Y. R. Schulz, "Postconditioning and protection from reperfusion injury: where do we stand? Position Paper from the Working Group of Cellular Biology of the Heart of the European Society of Cardiology," Cardiovasc. Res., vol. 87, no. 3, pp. 406-423, 2010.

[54] A. Finley Caulfield et al., "A comparison of cooling techniques to treat cardiac arrest patients with hypothermia," Stroke Res. Treat., vol. 2011, 2011.

[55] K. Kimble LP, "Perceived side effects and benefits of coronary angioplasty in the early recovery period," Hear. Lung, vol. 5, pp. 308-314, 1998.

[56] R. A. Kloner et al., "New and revisited approaches to preserving the reperfused myocardium," Nat. Publ. Gr., vol. 14, no. 11, pp. 679-693, 2017.

[57] R. A. Gerczuk, P. Z. \& Kloner, “An update on cardioprotection: a review of the latest adjunctive therapies to limit myocardial infarction size in clinical trials," $J$. Am. Coll. Cardiol., vol. 59, pp. 969-978, 2012.

[58] D. Hausenloy, "Targeting reperfusion injury in patients with ST-segment elevation myocardial infarction: trials and tribulations," Eur. Heart J., vol. 38, pp. 935-941, 2016.

[59] E. Iliodromitis, "What is wrong with cardiac conditioning? We may shoot moving targets," J. Pharmacol. Ther., vol. 20, pp. 357-369, 2015.

[60] A. Luz, "Lack of benefit of ischemic postconditioning after routine thrombus aspiration during reperfusion: immediate and midterm results," J. Cardiovasc. Pharmacol., vol. 20, pp. 523-531, 2015.

[61] J. M. Yang, X. M., Cui, L., Alhammouri, A., Downey and M. V. \& Cohen, "Triple therapy greatly increases myocardial salvage during ischemia/reperfusion in the in situ rat heart," Cardiovasc. Drugs Ther., vol. 27, pp. 403-412, 2013.

[62] C. W. Callaway et al., Part 8: Post-Cardiac Arrest Care, vol. 132. 2016.

[63] T. N. England, "Mild Therapeutic Hypothermia To Improve the Neurologic Outcome After Cardiac Arrest," English J., vol. 346, no. 8, pp. 549-557, 2002.

[64] S. Design, "Treatment of Comatose survivors of out-of-hospital cardiac arrest with induced hypothermia," vol. 346, no. 8, pp. 557-563, 2002.

[65] J. Varon and P. Acosta, "Therapeutic hypothermia: Past, present, and future," Chest, vol. 133, no. 5, pp. 1267-1274, 2008. 
[66] A. R. Henderson, “Cold--man's assiduous remedy,” Med. Ann. Dist. Columbia, vol. 40, p. 583, 1971.

[67] "Hippocrates," Vetere Med.

[68] M. D. Donald W. Benson, M.D., G. Rainey Williams, Jr., M.D., Frank C. Spencer, M.D., Adolph J. Yates, "The Use of Hypothermia After Cardiac Arrest," Anasthesia Analg., vol. 38, no. 6, pp. 423-428, 1959.

[69] W. G. Bigelow, J. C. Callaghan, and J. A. Hopps, "General Hypothermia for Experimental Intracardiac Surgery*," Ann. Surg., vol. 132, no. 3, pp. 531-537, 1950.

[70] W. C. Sealy, "Hypothermia: Its possible role in cardiac surgery," Ann. Thorac. Surg., vol. 47, no. 5, pp. 788-791, 1989.

[71] N. E. SHUMWAY and F. J. LEWIS, "Induced ventricular fibrillation for experimental intracardiac surgery under hypothermia," Ann. Surg., vol. 143, no. 2, pp. 230-234, 1956.

[72] M. D. Henry Swan, M.D. and Irvin Zeavin, "Cessation of circulation in general hypothermia. III. technics of intracardiac surgery under direct vision," Ann. Surg., vol. 139, no. 4, pp. 385-396, 1951.

[73] F. Nolan JP, Soar J, Cariou A, Cronberg T, Moulaert VR, Deakin CD, Bottiger BW and S. K. and S. C. H, "European Resuscitation Council and European Society of Intensive Care Medicine 2015 guidelines for post-resuscitation care," Intensive Care Med, vol. 41, pp. 2039-56, 2015.

[74] D. J. and B. A. Tissier R, Chenoune M, Ghaleh B, Cohen MV, "The small chill: mild hypothermia for cardioprotection?," Cardiovasc Res, vol. 88, pp. 406-414, 2010.

[75] A. Van Der Horst, F. L. Boogaard, M. Van'T Veer, M. C. M. Rutten, N. H. J. Pijls, and F. N. Van De Vosse, "Towards patient-specific modeling of coronary hemodynamics in healthy and diseased state," Comput. Math. Methods Med., vol. 2013, 2013.

[76] C. Y. Kim et al., "Case of recurrent ventricular fibrillations with osborn wave developed during therapeutic hypothermia," Korean Circ. J., vol. 45, no. 1, pp. 81-84, 2015.

[77] R. Dash et al., "Dose-Dependent Cardioprotection of Moderate $\left(32^{\circ} \mathrm{C}\right)$ Versus Mild $\left(35^{\circ} \mathrm{C}\right)$ Therapeutic Hypothermia in Porcine Acute Myocardial Infarction," JACC Cardiovasc. Interv., vol. 11, no. 2, pp. 181-191, 2018. 
[78] M. Dae et al., "Effects of endovascular cooling on infarct size in ST-segment elevation myocardial infarction: A patient-level pooled analysis from randomized trials," J. Interv. Cardiol., vol. 31, no. 3, pp. 269-276, 2018.

[79] W. Dai, M. J. Herring, S. L. Hale, and R. A. Kloner, "Rapid Surface Cooling by ThermoSuit System Dramatically Reduces Scar Size, Prevents Post-Infarction Adverse Left Ventricular Remodeling, and Improves Cardiac Function in Rats," $J$. Am. Heart Assoc., vol. 4, no. 7, pp. 1-9, 2015.

[80] C. W. Hoedemaekers, M. Ezzahti, A. Gerritsen, and J. G. van der Hoeven, "Comparison of cooling methods to induce and maintain normo- and hypothermia in intensive care unit patients: A prospective intervention study," Crit. Care, vol. 11, no. 4, pp. 1-9, 2007.

[81] C. Testori et al., "Strategic target temperature management in myocardial infarction - A feasibility trial," Heart, vol. 99, no. 22, pp. 1663-1667, 2013.

[82] L. Abella, J. W. Rhee, K.N. Huang, T.L. Vanden Hoek, "Induced hypothermia is underused after resuscitation from cardiac arrest: a current practice survey," Resuscitation, vol. 64, pp. 181-186, 2005.

[83] M. Merchant, J. Soar, "Therapeutic hypothermia utilization among physicians after resuscitation from cardiac arrest," Crit. Care Med., vol. 34, no. 7, pp. 1935-1940, 2006.

[84] A. R. Steinberg GK, Ogilvy CS, Shuer LM, Connolly ES Jr, Solomon RA, Lam A, Kassell NF, Baker CJ, Giannotta SL, Cockroft KM, Bell-Stephens TE, "Comparison of endovascular and surface cooling during unruptured cerebral aneurysm repair," Neurosurgery, vol. 55, no. 2, pp. 307-314, 2004.

[85] C. Flint, J. C. Hemphill, "Therapeutic hypothermia after cardiac arrest: performance characteristics and safety of surface cooling with or without endovascular cooling," Neurocrit. Care, vol. 7, no. 2, pp. 109-118, 2007.

[86] R. Gillies, R. Pratt, C. Whiteley, J. Borg and and S. M. Tibby, "Therapeutic hypothermia after cardiac arrest: a retrospective comparison between surface cooling and endovascular cooling," Resuscitation, vol. 81, pp. 1117-1122, 2010.

[87] I. S. Kang, I. Fumiaki, and W. B. Pyun, "Therapeutic hypothermia for cardioprotection in acute myocardial infarction," Yonsei Med. J., vol. 57, no. 2, pp. 291-297, 2016.

[88] M. Götberg et al., "A pilot study of rapid cooling by cold saline and endovascular cooling before reperfusion in patients with ST-elevation myocardial infarction," Circulation: Cardiovascular Interventions, vol. 3, no. 5. pp. 400-407, 2010. 
[89] N. B. Spath, N. L. Mills, and N. L. Cruden, "Novel cardioprotective and regenerative therapies in acute myocardial infarction: a review of recent and ongoing clinical trials," Future Cardiol., vol. 12, no. 6, pp. 655-672, 2016.

[90] K. Reddy, "Recent advances in the diagnosis and treatment of acute myocardial infarction," World J. Cardiol., vol. 7, no. 5, p. 243, 2015.

[91] L. O. Chavez, M. Leon, S. Einav, and J. Varon, "Editor's Choice- Inside the cold heart: A review of therapeutic hypothermia cardioprotection," Eur. Hear. J. Acute Cardiovasc. Care, vol. 6, no. 2, pp. 130-141, 2015.

[92] T. L. Merrill, J. E. Mitchell, and D. R. Merrill, "Heat transfer analysis of catheters used for localized tissue cooling to attenuate reperfusion injury," Med. Eng. Phys., vol. 38 , no. 8, pp. 758-766, 2016.

[93] T. L. Merrill, D. R. Merrill, T. J. Nilsen, and J. E. Akers, "Design of a Cooling Guide Catheter for Rapid Heart Cooling," J. Med. Device., vol. 4, no. 3, p. 035001, 2010 .

[94] T. L. Merrill, D. R. Merrill, and J. E. Akers, "Improved Ease of Use Designs for Rapid Heart Cooling," J. Med. Device., vol. 6, no. 3, p. 035001, 2012.

[95] L. C. Otterspoor, M. Van'T Veer, L. X. Van Nunen, I. Wijnbergen, P. A. L. Tonino, and N. H. J. Pijls, "Safety and feasibility of local myocardial hypothermia," Catheter. Cardiovasc. Interv., vol. 87, no. 5, pp. 877-883, 2016.

[96] M. McGarvey et al., "A feasibility and safety study of intracoronary hemodilution during primary coronary angioplasty in order to reduce reperfusion injury in myocardial infarction," Catheter. Cardiovasc. Interv., vol. 91, no. 2, pp. 234-241, 2018.

[97] “Philips InnerCool TRx Endovascular System,” Philips Medical Co., 2011.

[98] M. M. Van Der Worp HB, Sena ES, Donnan GA, Howells DW, "Hypothermia in animal models of acute ischaemic stroke: a systematic review and meta-analysis," Brain, vol. 130, no. 12, pp. 3063-3074, 2007.

[99] L. C. Otterspoor et al., "Intracoronary hypothermia for acute myocardial infarction in the isolated beating pig heart," Am. J. Transl. Res., vol. 9, no. 2, pp. 558-568, 2017.

[100] P. KH, "Mechanisms of action, physiological effects, and complications of hypothermia," Crit. Care Med., vol. 37, pp. 186-202, 2009.

[101] R. T. Primoz Trunk, Borut Gersak, "Topical cardiac cooling - computer simulation of myocardial temperature changes," Comput. Biol. Med., vol. 33, no. 3, pp. 203214, 2003. 
[102] J. Abas Abdoli, George S. Dulikravich, Chandrajit Bajaj, David F. Stowe, "Human heart conjugate cooling simulation: Unsteady thermofluid-stress analysis," Int. J. Numer. Methods Biomed. Eng., vol. 30, no. 11, pp. 1372-1386, 2014.

[103] S. Salles, F. P. Salvucci, and D. Craiem, "A reconstruction platform for coronary arteries, finite element mesh generation and patient specific simulations," J. Phys. Conf. Ser., vol. 332, no. 1, 2011.

[104] M. Molavi, R. Mongrain, and O. Bertrand, "Non-Newtonian Hemodynamics and Shear Stress Distribution in Three Dimensional Model of Healthy and Stented Coronary Artery Bifurcation," COMSOL Conf. Bost., pp. 1-5, 2010.

[105] M. Guanglei Xiong, Peng Sun, Haoyin Zhou, Seongmin Ha, Bríain ó Hartaigh, Quynh A. Truong, "Comprehensive Modeling and Visualization of Cardiac Anatomy and Physiology from CT Imaging and Computer Simulations," IEEE Trans. Vis. Comput. Graph., vol. 23, no. 2, pp. 1014-1028, 2017.

[106] S. Kamangar et al., "Patient-specific 3D hemodynamics modelling of left coronary artery under hyperemic conditions," Medical and Biological Engineering and Computing, vol. 55, no. 8. pp. 1451-1461, 2017.

[107] H. Nickisch et al., "Learning patient-specific lumped models for interactive coronary blood flow simulations," Springer International Publishing Switzerland, vol. 9350. pp. 433-441, 2015.

[108] C. A. Taylor, T. A. Fonte, and J. K. Min, "Computational fluid dynamics applied to cardiac computed tomography for noninvasive quantification of fractional flow reserve: Scientific basis," J. Am. Coll. Cardiol., vol. 61, no. 22, pp. 2233-2241, 2013.

[109] M. Stańczyk, G. M. J. Van Leeuwen, and A. A. Van Steenhoven, "Discrete vessel heat transfer in perfused tissue - Model comparison," Phys. Med. Biol., vol. 52, no. 9, pp. 2379-2391, 2007.

[110] E. Lim, A. S. Wong, N. S. Ahmad, K. B. Tan, M. E. Ong, and J. W. Tan, "Review of the clinical evidence and controversies in therapeutic hypothermia for survivors of sudden cardiac death," Proc. Singapore Healthc., vol. 24, no. 1, pp. 42-53, 2015 .

[111] M. S. Shlykov V, Danilova V, Maksymenko V, "Application of Model of Heat Exchange for Miocardium Provided Stationary Convection Laminar Flow," vol. 10, no. 1, pp. 1-3, 2017.

[112] M. Sterk and R. Trobec, "Biomedical simulation of heat transfer in a human heart.," J. Chem. Inf. Model., vol. 45, pp. 1558-1563, 2005. 
[113] G. M. Van Leeuwen, A. N. Kotte, and J. J. Lagendijk, "A flexible algorithm for construction of 3-D vessel networks for use in thermal modeling," IEEE Trans. Biomed. Eng., vol. 45, no. 5, pp. 591-595, 1998.

[114] C. L. jiyuan Tu, Guan-Heng Yeoh, Computational Fluid Dynamics, Second. 2013.

[115] R. H. Yunus A. Cengel, John M. Cimbala, Fundamentals of Thermal-Fluid Sciences. McGraw-Hill Companies, 2012.

[116] H. N. Hemida, M. N. Sabry, A. Abdel-Rahim, and H. Mansour, "Theoretical analysis of heat transfer in laminar pulsating flow," Int. J. Heat Mass Transf., vol. 45, no. 8, pp. 1767-1780, 2002.

[117] A. D. Barozzi, "Convective heat transfer coefficients in the circulation," J. Biomech. Eng., vol. 113, no. 3, pp. 308-313, 1991.

[118] Y. Zhang and H. Xie, "The effect of a bifurcation structure on the heat transfer and temperature distribution of pulsatile blood flow," Int. J. Heat Mass Transf., vol. 118, pp. 663-670, 2018.

[119] D. N. Xiaoyi He, "Pulsatile flow in the human left coronary artery bifurcation: average conditions," J. Biomech. Eng., vol. 118, no. 1, pp. 74-82, 1996.

[120] W. W. Bruce R. Munson, Donald F Young, Theodore H. Okiishi, Fundamentals of Fluid Mechanics, Sixth Edition. Wiley, 2009.

[121] A. H. Jean Donea, Finite Element Methods for Flow Problems. Wiley \& Sons, Ltd, 2003.

[122] Comsol, "Nonisothermal Flow," Multiphysics Cyclopedia. 2017.

[123] D. M. Eckmann, S. Bowers, M. Stecker, and A. T. Cheung, "Hematocrit, volume expander, temperature, and shear rate effects on blood viscosity," Anesth. Analg., vol. 91, no. 3, pp. 539-545, 2000.

[124] Q. Nguyen and N. Nguye, "Incompressible Non-Newtonian Fluid Flows," Contin. Mech. - Prog. Fundam. Eng. Appl., 2012.

[125] U. Eberhard et al., "Determination of the Effective Viscosity of Non-newtonian Fluids Flowing Through Porous Media," Front. Phys., vol. 7, no. May, pp. 1-9, 2019.

[126] N. Materialise, “Mimics Student Edition Course Book,” pp. 1-83, 2010.

[127] V. DICOM, "Digital Imaging and Communications in Medicine," Medical Imaging \& Technology Alliance (MITA), 2018. 
[128] C. Matthew, “Aeronautical engineer's data book," Butterworth-Heinemann, vol. 2nd ed, p. 229, 2005.

[129] A. Inc, "Introduction to CFD Analysis," ANYSYS Training Manual, 2015.

[130] H. K. Versteeg and W. Malalasekera, An Introduction to Computational Fluid Dynamics. 2007.

[131] J. Blazek, "Computational Fluid Dynamics: Principles and Applications," Elsevier, Amsterdam, 2001.

[132] M. Ferziger, Joel H. and Peric, "Computational Methods for Fluid Dynamics," Springer, Berlin, 1999.

[133] SIEMENS PLM Software, "Simcenter STAR-CCM+.” 2018.

[134] William L. Oberkampf and T. G. Trucano, "AIAA Guide for the Verification and Validation of Computational Fluid Dynamics Simulations. AIAA G-077-1998 (2002)," Prog. Aerosp. Sci., vol. 38, no. March 2002, pp. 210-270, 2002.

[135] P. A. Iaizzo, "The Visible Heart® project and free-access website 'Atlas of Human Cardiac Anatomy,"' Europace, vol. 18, pp. iv163-iv172, 2016.

[136] B. A. Anderson RH, "The Heart: Structure in Health and Disease," London Gower Med. Publ., 1992.

[137] R. K. Weinhaus AJ, “Anatomy of the human heart. In: PA laizzo (ed). The Handbook of Cardiac Anatomy, Physiology, and Devices, 2nd ed," New York Humana Press, pp. 59-85, 2009.

[138] M. G. Maselli D, Guarracino F, Chiaramonti F, Mangia F, Borelli G, "Percutaneous mitral annuloplasty: an anatomic study of human coronary sinus and its relation with mitral valve annulus and coronary arteries," Circulation, vol. 114, pp. 377-380, 2006.

[139] laizzo P. Anderson SE, Quill JL, "Venous valves within left ventricular coronary veins," J. Interv. Card. Electrophysiol., vol. 23, pp. 95-99, 2008.

[140] I. P. Quill JL, Hill AJ, Laske TG, Alfieri O, "Mitral leaflet anatomy revisited," J. Thorac. Cardiovasc. Surg., vol. 137, pp. 1077-1081, 2009.

[141] Y. Y. Alcocer, "Surface Area to Volume Ratio," GMAT Prep: Help and Review.

[142] S. H. M. Beals, L. Gross, “The Surface Area: Volume Ratio," in Sizes Of Organisms, 2000. 
[143] A. Hamdan, P. Asbach, E. Wellnhofer, A. Huppertz, and E. Fleck, "A Prospective Study for Comparison of MR and CT Imaging for Detection of Coronary Artery Stenosis," JCMG, vol. 4, no. 1, pp. 50-61, 2011.

[144] "CardiologySite: Circumflex Coronary Artery,” A.S.M. Systems, 2010.

[145] T. Diorio, N. Bouhrira, M. Singh, and T. Merrill, "Computational Modeling of the Left Coronary Artery during Targeted Therapeutic Hypothermia Introduction Methods Results," vol. m, p. 723, 2017.

[146] C. Nahirnyak, S.W. Yoon, "Acousto-mechanical and thermal properties of clotted blood,” J. Acoust. Soc. Am., vol. 119, no. 6, pp. 3766-3772, 2006.

[147] F. Lockwood, L.K. Ryan, J.W. Hunt, "Measurement of the ultrasonic properties of vascular tissues and blood from 35-65 MHz," Ultrasound Med. Biol., vol. 17, no. 7, pp. 653-666, 1991.

[148] G. Poul-Erik Paulev, "Thermo-Regulation, Temperature and Radiation," in New Human Physiology, 2004, p. Chapter 21.

[149] C. H. "Microvascular contributions in tissue heat transfer," Ann. N. Y. Acad. Sci., pp. 137-154, 1980.

[150] M. C. McGoodwin, "The First Law of Thermodynamics," in Engineering Thermodynamics, 2016, pp. 23-25.

[151] M. CardioVascular, "CardioVascular Academia: Guide Catheter Selection.” pp. 194.

[152] G. M. Villablanca PA, Rao G, Briceno DF, Lombardo M, Ramakrishna H, Bortnick A and M. M. and M. F. Menegus M, Sims D, "Therapeutic hypothermia in ST elevation myocardial infarction: a systematic review and meta-analysis of randomised control trials," Heart, 2016.

[153] F. M. White, Seventh Edition Fluid Mechanics, Seventh Ed. McGraw-Hill Higher Education, 2010.

[154] R. B. Bird, W. E. Stewart, and E. N. Lightfoot, “Transport Phenomena I,” pp. 132, 2002.

[155] Richard Haberman, Applied Partial Differential Equations with Fourier Series Boundary Value Problems, Fifth. Pearson, 2013.

[156] P. A. A. Denise M. Zezell and P. R. C. and W. V. J. Thiago M. Pereira, "Heat Generation and Transfer on Biological Tissues Due to High-Intensity Laser Irradiation, Developments in Heat Transfer," InTech, 2011. 


\section{Appendix A}

\section{Simplified explanation of fully developed velocity profile, energy balance, and the temperature dependent heat source equation}

\section{Velocity Profile Equation}

For incompressible, fully developed laminar flow in a horizontal circular pipe, the fluid properties will be assumed constant, and flow will move at a constant velocity along the axial length of the pipe. Thus, velocity $u(r)$ is represented as a steady parabolic profile and will remain constant in the direction of flow (Fig. A.1).

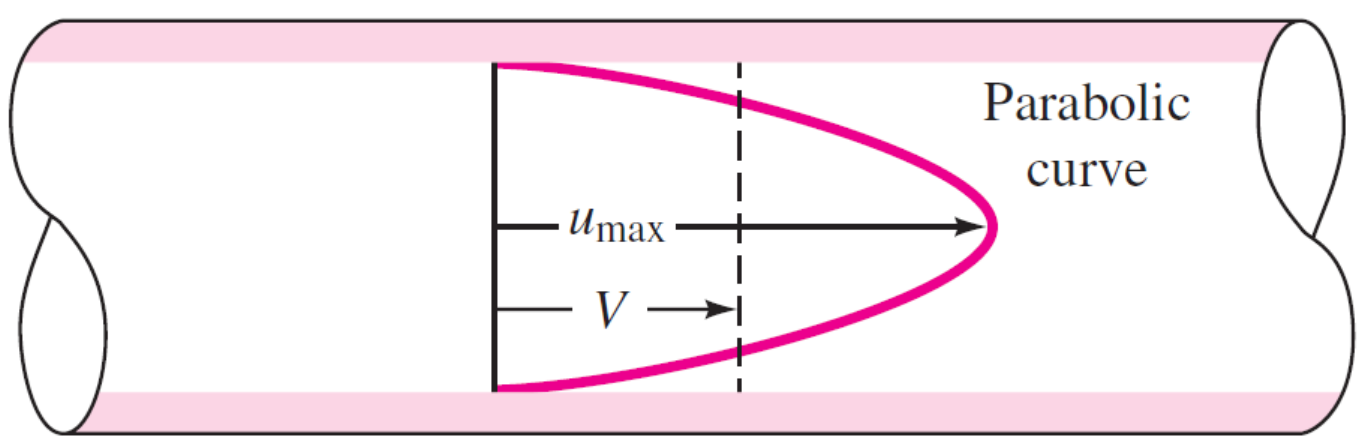

Figure A.1. The velocity profile for fully developed laminar flow in a pipe with radius $\mathrm{R}$ and length $\mathrm{L}$. At $\mathrm{R}=0$ at the centerline, the velocity is maximum, $v_{\max }$. The velocity profile is constant with respect to $\mathrm{z}$ and $\theta$, the coordinate angle of rotation into the page [153].

The radial direction has no fluid motion; thus, the velocity is zero everywhere normal to the direction of flow. Since flow is only varying in the r-direction, flow in the $\theta$ and $\mathrm{z}$ direction is neglected, and velocity $\frac{\partial \vec{v}}{\partial t}$ is ignored due to steady state flow. Presuming there are no forces acting on the fluid, the velocity equation in a pipe becomes: 


$$
\frac{1}{r} \frac{\partial}{\partial r}\left(r \frac{\partial v_{z}}{\partial r}\right)=\frac{1}{\mu} \frac{\partial p}{\partial z}
$$

Since the pressure will decrease linearly along the length of a pipe, the pressure gradient, $\frac{\partial p}{\partial z}$, is considered a constant. Integrating equation A. 1 with respect to the radius yields the velocity profile as a function of the pipe radius, where $v_{z}$ is the fluid velocity in the z-direction. To solve for a finite $v_{z}$ at the center of the pipe $(r=0)$, the velocity must be zero at the wall $(r=R)$ to satisfy the no-slip boundary condition. Thus, when $r=0, \frac{\partial v_{z}}{\partial r}$ is zero and $\mathrm{v}_{\mathrm{z}}$ is maximum.

The final velocity profile equation for steady, laminar flow in an axisymmetric pipe becomes:

$$
v_{z}=\frac{1}{4 \mu}\left(\frac{\partial p}{\partial z}\right)\left(r^{2}-R^{2}\right)
$$

where $\mu$ is the viscosity, $\mathrm{R}$ is the radius of the pipe, and the velocity distribution in the pipe is parabolic at any cross section. A relationship can be obtained between the pressure gradient, $\frac{\partial p}{\partial z}$, and volumetric flow rate, $Q$, by integrating over a finite differential area, $d A=(2 \pi r) d r$, in the pipe. The resulting flowrate, $Q$, through the pipe becomes the Hagen-Poiseuille equation [153]:

$$
Q=\frac{\pi R^{4} \Delta p}{8 \mu l}
$$

where the volumetric flow rate, $Q$, is proportional to the radius, $\mathrm{R}$, to the fourth power and inversely proportional to viscosity, $\mu$.

\section{Energy Balance - Governing Equations}

The following derivation is a mathematical model used to predict the temperature profile of a non-isothermal laminar fluid through a simple pipe with constant wall temperatures. The derived equation will predict flow through a tubular pipe with constant 
heated walls to determine the pipes outlet temperatures. The energy balance for a horizontal pipe system with heat and work [154] can be described as,

$$
q-w+\sum E_{\text {in }}-\sum E_{\text {out }}=\frac{d}{d t}\left(E_{\text {sys }}\right)
$$

where $\mathrm{q}$ is the convective heat flux from the surface of the pipe into the fluid, $\mathrm{w}$ is work, and $E_{\text {sys }}$ is the total energy change in the computational domain of the pipe with respect to time.

To apply (A.4) to an energy balance for steady-state, laminar fluid flow in a simple horizontal pipe system with constant wall temperatures, there is no work being done, so $w$ $=0$, and the energy balance becomes an unsteady-state partial differential equation (PDE) with the form [155]:

$$
\frac{\partial E}{\partial t}=\frac{-\partial\left(C_{p} T_{A B T} \dot{m}\right)}{A_{c} \Delta Z}+h\left(T_{w}-T_{i n}\right) \frac{2}{R}
$$

where $C_{p}$ is the specific heat, $T_{A B T}$ represents the difference in average bulk temperature (ABT) of the fluid across the length of the pipe, $\dot{m}$ is the mass flow rate, $A_{c}$ is the crosssectional area of the pipe, $h$ is the heat transfer coefficient, $T_{w}$ is the temperature of the wall, and $T_{\text {in }}$ is the ABT entering the pipe.

Assuming steady-state, $\frac{\partial E}{\partial t}$ in equation (A.5) goes to zero, and if we combine like terms, integrate with respect to temperature from $T_{\text {in }}$ to $T_{\text {Out }}$ across the length of the pipe, and rearrange to solve for $T_{\text {Out }}$, the final analytical equation for the steady-state temperature profile becomes:

$$
T_{\text {Out }}=T_{w}-\left(T_{w}-T_{\text {in }}\right) e^{\left(-\frac{h 2 \pi R}{C_{p} \dot{m}^{m}} z\right)}
$$

where $T_{\text {Out }}$ is the $\mathrm{ABT}$ at the outlet of the pipe, $\mathrm{R}$ is the radius, and $\mathrm{z}$ is the length of the pipe. 


\section{Temperature dependent heat source equation}

A common model used to determine the temperature difference between the surface of a pipe and its internal fluid flow is the temperature dependent heat source equation [156]:

$$
Q_{c}=\dot{m}_{c} C_{p, c} \Delta T_{c}
$$

where $Q_{c}$ is the cooling capacity and is dependent on the mass flow rate, $\dot{m}_{c}$, the specific heat, $C_{p, c}$, and the difference between the pipe surface temperature and the outlet temperature, $\Delta T_{c}$.

In Eq. (A.7), the heat flux, $Q_{c}$, can also be described as the heat conduction rate, $\dot{q}$, per unit area, per unit time, and is defined as Fourier's Law of Cooling [156] for a onedimensional plane:

$$
\dot{q}=-k \frac{\Delta T_{w}}{\Delta x}
$$

where $-k$ is the thermal conductivity representing the heat-flux moving from high to low temperatures, $\Delta T_{w}$ is the change in temperature between the outer and inner wall, and $\Delta x$ is the thickness of the wall. 


\section{Appendix B}

\section{Description of viscosity entered as function of temperature in STAR-CCM+}

As discussed in Chapter 2, Equation 2.11, was used to determine the dynamic viscosity as a function of mixed temperature $T_{m}\left({ }^{\circ} \mathrm{C}\right)$, shear rate $\Upsilon\left(\frac{1}{s}\right)$, and hematocrit (Hct (\%)) for each GuideLiner® flow rate [123].

$$
\mu\left(\Upsilon, \text { Hct }, T_{m}\right)=\left(e^{\frac{\lambda}{\Upsilon+\eta \cdot H c t}}\right)\left[\alpha+\frac{\phi}{\left(1+e^{\beta(T-\epsilon)}\right)}\right]
$$

Equation 2.11 was used to solve for dynamic viscosity in each of the thirty-six CFD simulations. The ABT at each discrete location along the fluid flow path was plotted vs Eq. 2.11 to yield a non-linear decreasing slope (Fig. B.1).

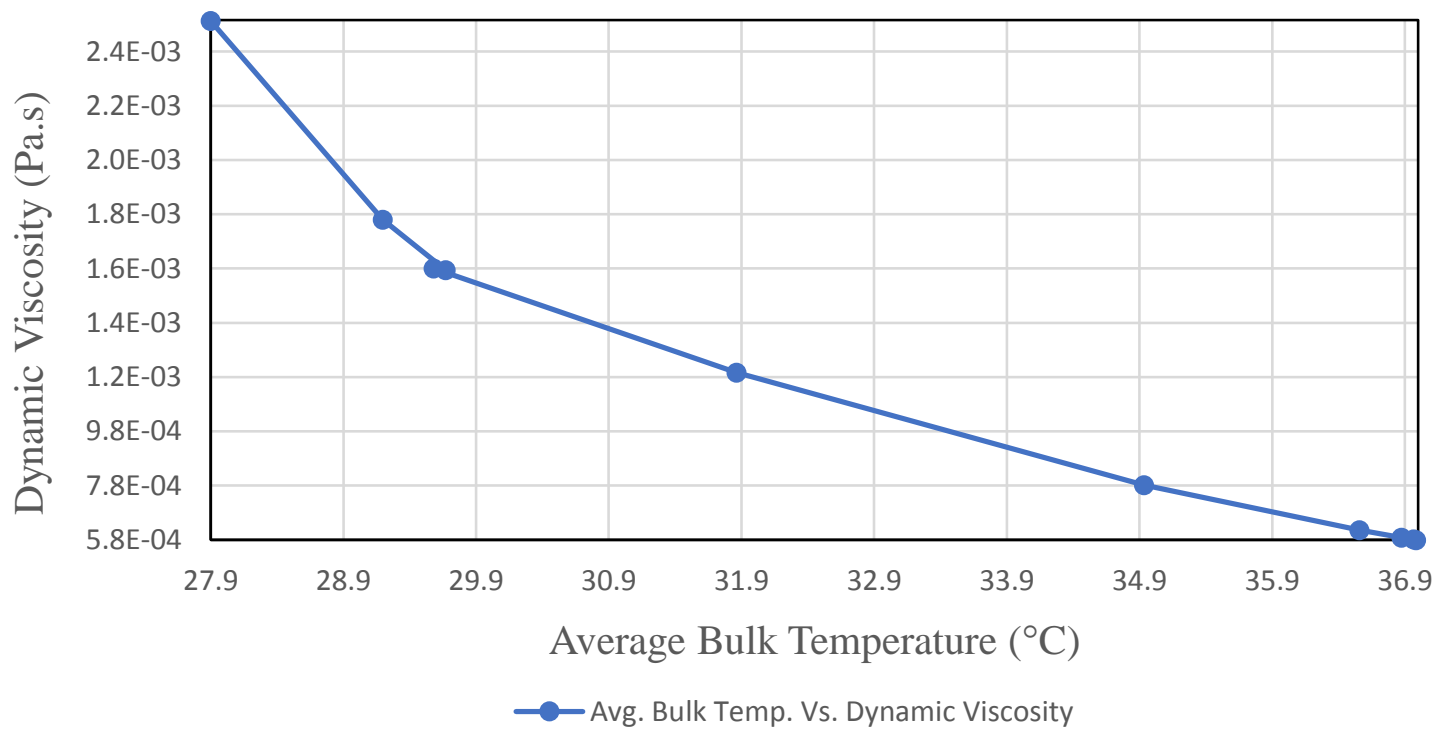

Figure B.1. ABT along the fluid flow path for HH63 LAD vs dynamic viscosity using Eq. 2.11. 
A Polynomial in T function was used to replicate the slope of Fig. B.1 to represent the independent variables (shear rate, hematocrit, and temperature) and the dependent variable (dynamic viscosity) from Eq. 2.11 and were entered as a function of temperature in STAR-CCM+ (Fig. B.2).

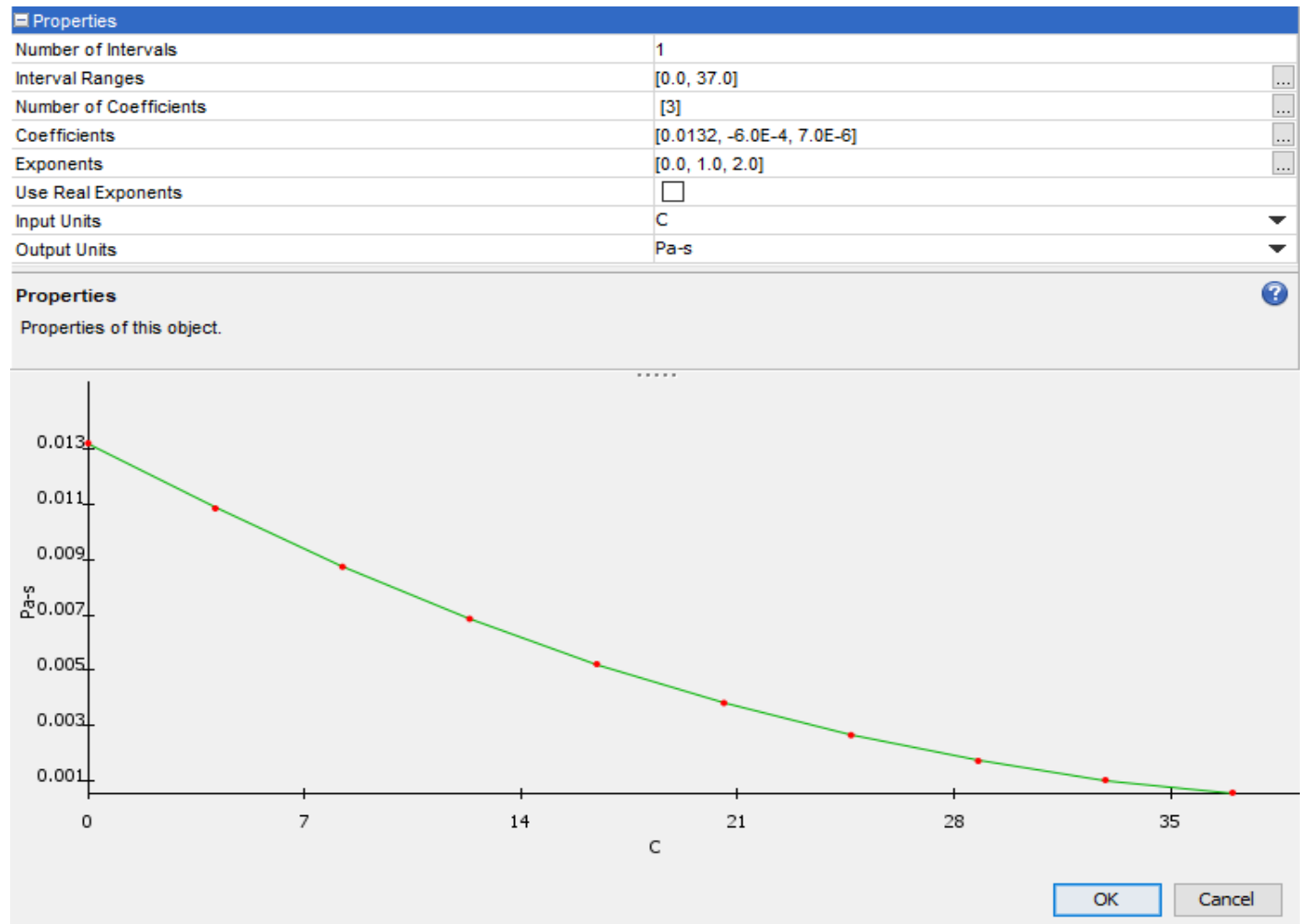

Figure B.2. Equation 2.11 applied as a Polynomial in T function in STAR-CCM+.

The slope from Figure $2 b$ is an extrapolation of Figure $2 \mathrm{a}$ which was entered as a function in STAR-CCM+ and applied to all thirty-six patient-specific CFD simulations. A constant viscosity at $32.5^{\circ} \mathrm{C}$ was for the $28.5 \mathrm{~mL} / \mathrm{min}$ GuideLiner ${ }^{\circledR}$ flow rate was plotted with a polynomial in $\mathrm{T}$ function against temperature along the fluid flow path of HH63 LAD (Fig. B.3). 


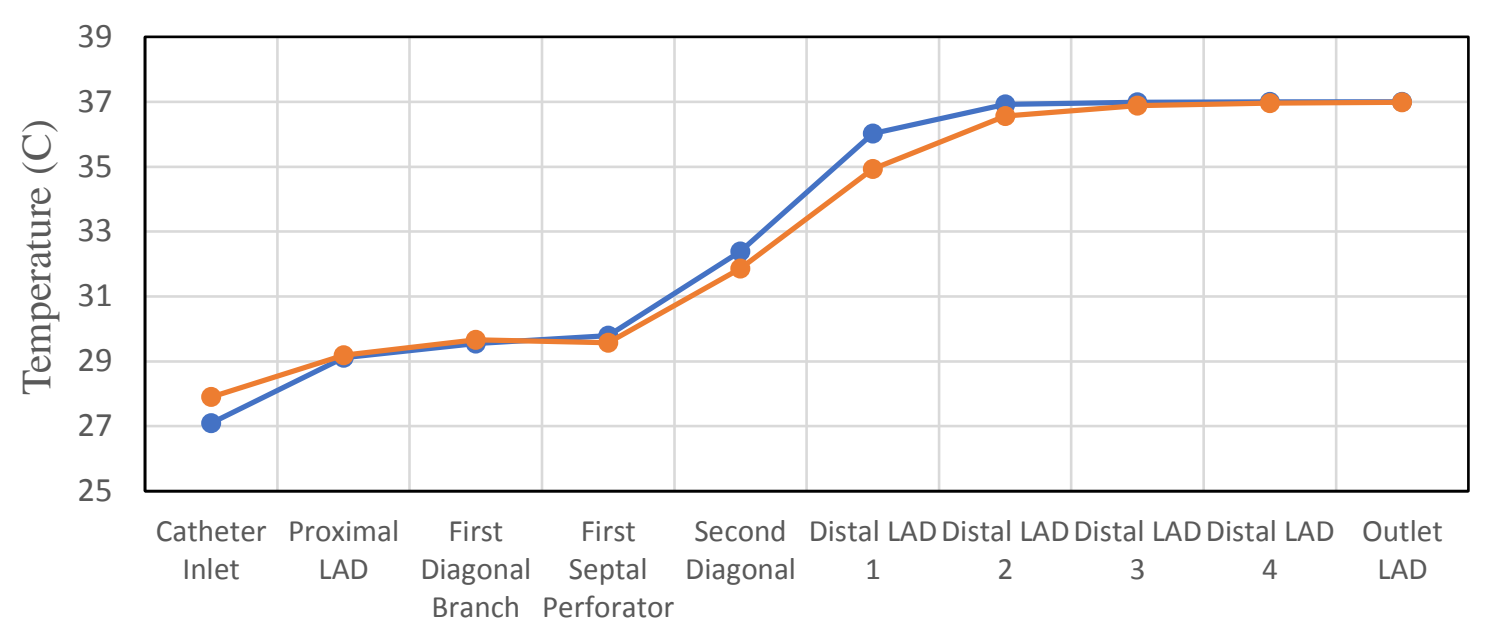

Location Along LAD Artery Blood Pathway

$\longrightarrow$ —Constant Viscosity @ $28.5 \mathrm{ml} / \mathrm{min}$ using mu @ 32.5 C $\rightarrow$ Polynomial in T @ $28.5 \mathrm{ml} / \mathrm{min}$

Figure B.3. ABT at discrete locations along HH63 LAD fluid flow path at $29.2 \mathrm{~mL} / \mathrm{min}$ GuideLiner® flow rate.

The Polynomial in T data fit the constant viscosity assumption fit fairly well with very little variation in the trendline from the GuideLiner® inlet to the LAD outlet. 


\section{Appendix C}

\section{Extension of the thermal fluid modeling matrix specifications}

An extension of the thermal fluid model testing matrix is herein described for each patient-specific representative heart model. A more comprehensive version of the specifications are described in detail in the following tables - courtesy of the Atlas of Human Cardiac Anatomy from the Visible Heart Lab, University of Minnesota.

Figures C. 1 - C.4 illustrate an extension of the thermal fluid modeling matrix of heart histories and specimen demographics. The information from Figure C.1 is freely available on the Atlas of Human Cardiac Anatomy website (Link:

http://www.vhlab.umn.edu/atlas/). 


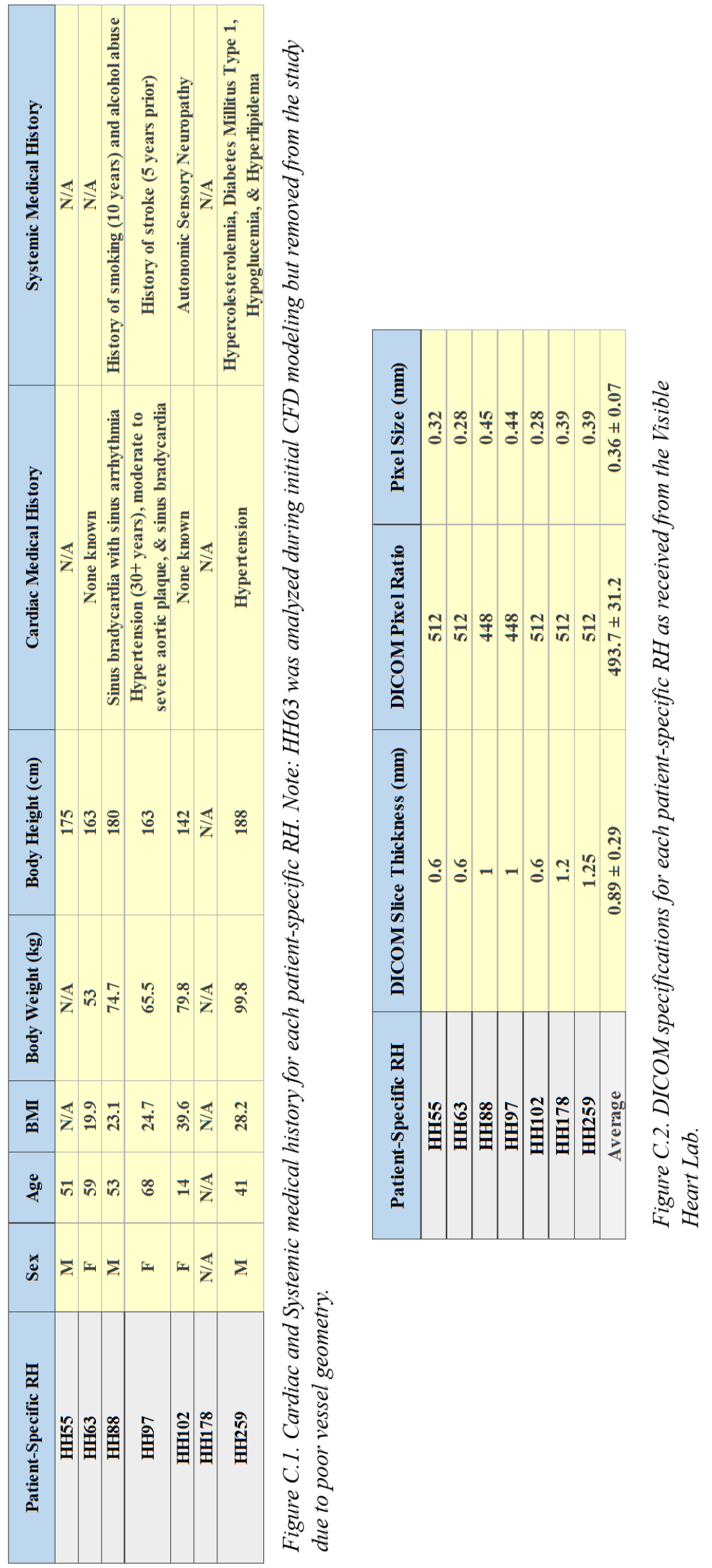



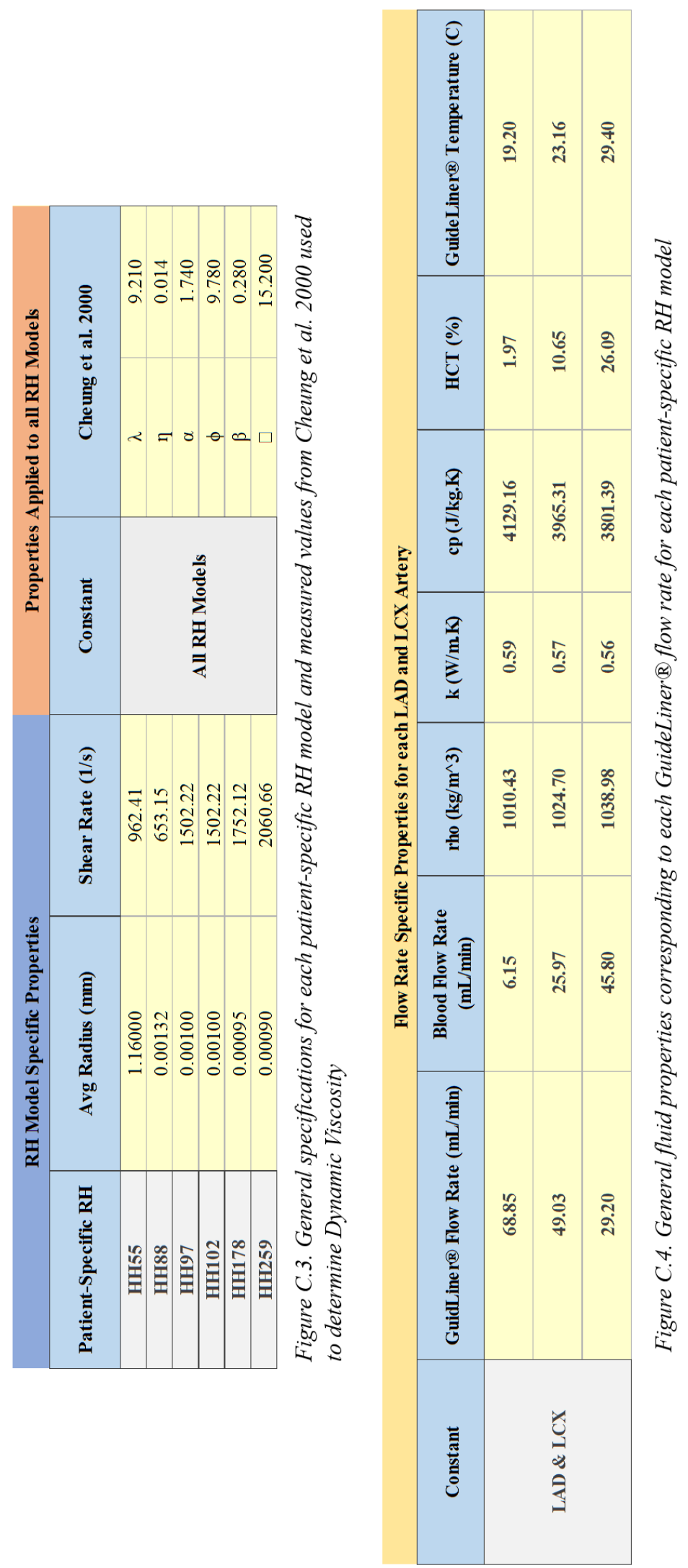


\section{Appendix D}

\section{Qualitative analysis of how ABT changes along the fluid flow path using simple 3D tube flow in STAR-CCM+}

As illustrated in Chapter 7, ABTs increased along the length of the arteries and experienced dramatic increases in ABT where vessels branched or artery diameters significantly reduced in size. For the simple 3D tube case, however, a slight increase in ABT is evident between the Proximal 8 and Mid 1 planes (Fig. D.1).

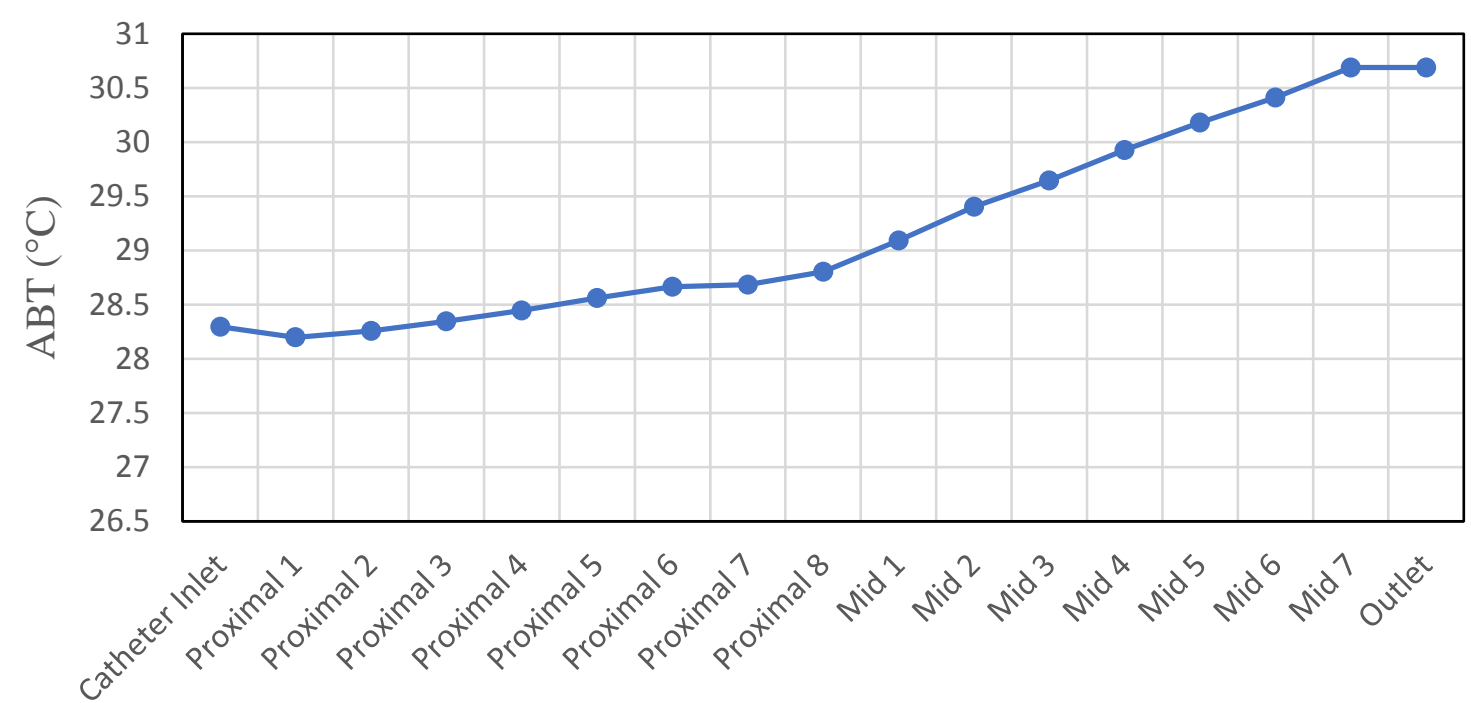

ABT cross-sectional plane location along 3D tube

Figure D. 1. ABT cross-sectional planes along 3D tube for simple pipe flow using 28.5 $\mathrm{mL} / \mathrm{min}$ GuideLiner® flow rate at $13.33^{\circ} \mathrm{C}$.

The sudden increase in slope between the Proximal 8 and Mid 1 cut planes was due to a 3x gap extension implemented for the remaining Mid cut planes. The extended gap between cut planes and contour ABT plots along the fluid path of the 3D tube are illustrated in Fig. D.2. 


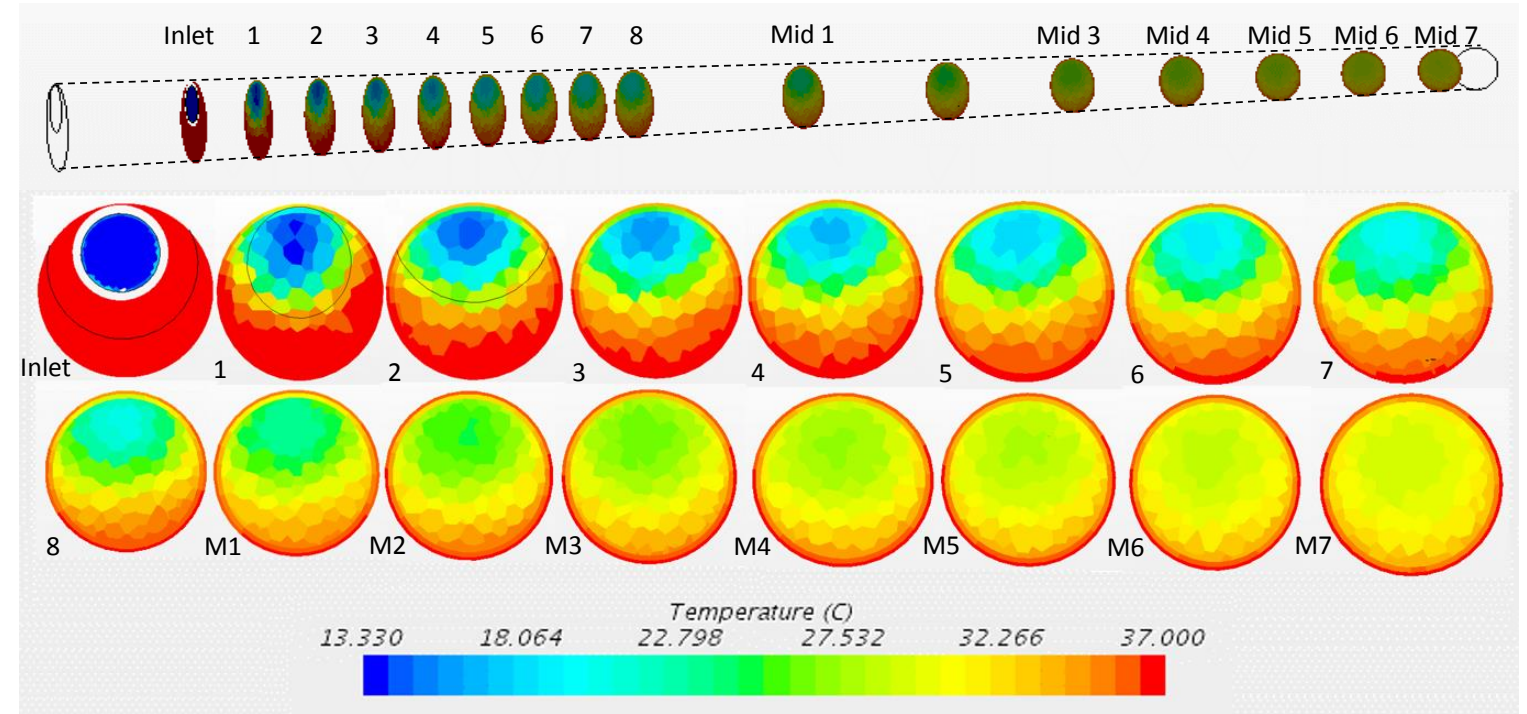

Figure D.2. Contour plots of the ABT cross-sectional planes as illustrated in Fig. 3a at each location along the length of the simple 3D tube. Mixing between the chilled saline (blue) and blood (red) increases as flow continues down the length of the tube, with the most mixing occurring towards the outlet, as seen in the Mid 7 (M7) contour plot.

Figure D.2 illustrates the mixing effect between GuideLiner® and blood flow by considering the temperature contours at each cross sectional plane within the tube. To visualize the flow field and temperature distribution within each artery vessel, each fluid is colored with respect to temperature, with chilled distilled water (blue) and blood (red). It is evident that mixing and rewarming occurs downstream in the tube since the majority of the contour color falls within yellow and green temperatures $\left(25-33^{\circ} \mathrm{C}\right)$, which is much higher than the temperature at the GuideLiner® inlet $\left(13.33^{\circ} \mathrm{C}\right)$. Since this method was applied to each RH model, custom cross-sectional planes were created along each fluid flow path so that temperature distributions could be investigated for each patient-specific vascular network. 